\title{
Characterization and Processing of Atrial Fibrillation Episodes by Convolutive Blind Source Separation Algorithms and Nonlinear Analysis of Spectral Features
}

\section{Caracterización y Procesado de Episodios de Fibrilación Auricular Mediante Algoritmos Convolutivos de Separación Ciega de Fuentes y Análisis No Lineal de Características Espectrales}

\author{
Carlos Vayá Salort
}

TUTOR: José Joaquín Rieta Ibáñez

Valencia, April 2010

Doctoral Thesis

DEPARTMENT OF ELECTRONIC ENGINEERING

UNIVERSIDAD POLITÉCNICA DE VALENCIA

UNIVERSIDAD

POLITECNICA

DE VALENCIA 
To my family, especially to my wife Silvia.

I am very grateful to all people who gave me support throughout this work. 



\section{Abstract}

Supraventricular tachyarrhythmias, in particular Atrial Fibrillation (AF), are the most commonly cardiac diseases encountered in the routine clinical practice. The prevalence of AF is less than $1 \%$ among population under 60 years old, but it increases significantly among those over 70 , approximating to $10 \%$ in those older than 80 . Undergoing a sutained AF episode is related to a higher mortality ratio and to a rising probability of suffering thromboembolisms, myocardial infarction, and stroke. On the other hand, paroxysmal AF (PAF), which is characterized by its spontaneous termination, is frequently the precursor to sustained AF. This provokes a great interest among the scientific community in disclosing the mechanisms which lead to AF perpetuation or to spontaneous AF termination.

The analysis of the surface electrocardiogram (ECG) is the most extended noninvasive technique in medical diagnosis of cardiac pathologies. In order to use the ECG as a tool for the AF study, the atrial activity (AA) must be separated from other cardioelectric signals. In this sense, Blind Source Separation (BSS) techniques are able to perform a multi-lead statistical analysis with the aim to obtain a set of independent sources that include the AA. When the BSS problem is tackled, it becomes necessary to consider a source mixing model near to the real mixing process in order to develop mathematical algorithms that solve the problem. A feasible model consists of assuming the linear mixture of sources. Within this linear mixing model it can be made the additional assumption of instantaneous mixture. This instantaneous linear mixing model is the one used in Independent Component Analysis (ICA). An alternative mixing model is considered by convolutive BSS (CBSS) algorithms, where a more realistic process in the generation of ECG leads is taken into account with delayed contributions of cardioelectric sources.

In this thesis, a performance study of CBSS algorithms applied to AA extraction from ECG recordings has been carried out for the first time. With this aim, the most relevant CBSS algorithms have been compared with the instantaneous algorithm FastICA, the effectivity of which is extensively proved. This comparison will allow to know which CBSS algorithms are useful for AA extraction from ECG recordings of AF episodes. On the other hand, CBSS algorithms have the problem of requiring a minimum number of observed signals for their suitable 
application. Here a new AA extraction algorithm is presented, which is based on the convolutive mixing model and solves the problem of lack of available leads from Holter ECG recordings. The high likeliness level between original and estimated AA, measured by different performance indicators, demonstrates the suitability of this new method for the AA extraction from Holter recordings and, furthermore, a higher robustness against noise of the convolutive mixing model is highlighted.

The most common cause of undergoing an AF episode is attributed to the reentry mechanism, which consists of multiple wavelet fronts that propagate through the atrial tissue. Recent studies have proved a relation between the number of simultaneous reentries and atrial electrical activity organization of the atria. In addition, it is also known the progressive deterioration of the atrial electrical activity organization after the PAF onset and the organization increase prior to its return to normal sinus rhythm. Furthermore, the maintenance of AF episodes is related to frequency dispersion variability of AA. From a clinic point of view, it is interesting to evaluate the atrial electrical activity by means of regularity indicators applied to the AA extracted from the surface ECG with the aim to predict the evolution of PAF episodes. This can be carried out from two different perspectives. Firstly, a regularity estimation can be applied to the raw AA obtained by extraction algorithms. An alternative way to tackle the problem is to study the regularity of certain spectral feature throughout time. This last aspect has never been considered before and is a matter of study in this thesis.

The work of this thesis finishes with the presentation of a new method for predicting the early termination or the maintenance of PAF episodes. This new method is based on the regularity analysis of AA spectral features. During the method design, the regularity of twelve numerical series of spectral features was analyzed. In order to construct these series, the spectrogram of the AA was previously computed. The series regularity was estimated by the nonlinear regularity estimator sample entropy (SampEn). The SampEn of six spectral features were revealed as statistically relevant to PAF characterization with $p<0.05$ for all features. This study was complemented with a multivariate regularity analysis that executes a joint study of the spectral features series and the AA in time domain The multivariate analysis discloses the combination of features that optimizes the prediction with $100 \%$ of correctly classified recordings for the learning set and $93.33 \%$ for the test set. Consequently, the presented method can be reliably used for predicting PAF termination. 


\section{Resumen}

Las arritmias supraventriculares, en particular la fibrilación auricular (FA), son las enfermedades cardíacas más comúnmente encontradas en la práctica clínica rutinaria. La prevalencia de la FA es inferior al 1\% en la población menor de 60 años, pero aumenta de manera significativa a partir de los 70 años, acercándose al 10\% en los mayores de 80 . El padecimiento de un episodio de FA sostenida, además de estar ligado a una mayor tasa de mortalidad, aumenta la probabilidad de sufrir tromboembolismo, infarto de miocardio y accidentes cerebrovasculares. Por otro lado, los episodios de FA paroxística, aquella que termina de manera espontánea, son los precursores de la FA sostenida, lo que suscita un alto interés entre la comunidad científica por conocer los mecanismos responsables de perpetuar o conducir a la terminación espontánea de los episodios de FA.

El análisis del ECG de superficie es la técnica no invasiva más extendida en la diagnosis médica de las patologías cardíacas. Para utilizar el ECG como herramienta de estudio de la FA, se necesita separar la actividad auricular (AA) de las demás señales cardioeléctricas. En este sentido, las técnicas de Separación Ciega de Fuentes (BSS) son capaces de realizar un análisis estadístico multiderivación con el objetivo de recuperar un conjunto de fuentes cardioeléctricas independientes, entre las cuales se encuentra la AA. A la hora de abordar un problema de BSS, se hace necesario considerar un modelo de mezcla de las fuentes lo más ajustado posible a la realidad para poder desarrollar algoritmos matemáticos que lo resuelvan. Un modelo viable es aquel que supone mezclas lineales. Dentro del modelo de mezclas lineales se puede además hacer la restricción de que estas sean instantáneas. Este modelo de mezcla lineal instantánea es el utilizado en el Análisis de Componentes Independientes (ICA). Un modelo de mezcla alternativo es el considerado por los algoritmos BSS convolutivos (CBSS), donde se tiene en cuenta un proceso de generación más realista de las derivaciones de ECG con contribuciones retardadas de fuentes cardioeléctricas.

En esta tesis se ha realizado por primera vez un estudio de rendimiento de los algoritmos CBSS aplicados a la extracción de la AA a partir de registros de ECG. Para ello, se han comparado los algoritmos CBSS más relevantes con el algoritmo instantáneo FastICA, cuya efectividad está ya ampliamente contrastada. Esta comparación va a permitir saber qué algoritmos CBSS resultan ser útiles 
para extraer la AA de los registros ECG de episodios de FA. Por otro lado, los algoritmos CBSS presentan el problema de requerir un mínimo número de señales observadas para su adecuada aplicación. Aquí se presenta un nuevo algoritmo para la extracción de la AA, basado en el modelo convolutivo de mezcla, que resuelve el problema de la escasez de derivaciones que poseen los registros de Holter. El alto grado de similitud entre la AA original y la AA estimada, medido por los dsitintos indicadores de rendimiento utilizados, demuestra la idoneidad del nuevo método presentado para la extracción de la AA a partir de registros Holter, además de poner de relieve una mayor robustez frente al ruido del modelo convolutivo de mezcla.

La causa más común que explica la aparición de episodios de FA es el mecanismo de reentrada, que consiste en a la existencia de múltiples frentes de propagación que recorren el tejido auricular. Estudios recientes han demostrado la relación existente entre el número de reentradas simultáneas y la organización de la actividad eléctrica de las aurículas. Por otra parte, también son conocidos el progresivo deterioro de la organización de la actividad eléctrica auricular tras el comienzo de la FA paroxística y el incremento de la misma en los instantes previos al retorno a ritmo sinusal normal. El mantenimiento de los episodios de FA está relacionado además con la variabilidad de dispersión en frecuencia de la AA. Desde un punto de vista clínico, resulta interesante evaluar el nivel de organización eléctrica de las aurículas mediante indicadores de regularidad aplicados a la AA extraída del ECG de superficie con el objetivo de predecir la evolución de la FA paroxística. Esto se puede llevar a cabo desde dos perspectivas distintas. La primera forma de hacerlo sería aplicar la estimación de regularidad a la señal de AA tal como la obtienen los métodos de extracción. Una manera alternativa de abordar el problema sería estudiar la regularidad de ciertas características espectrales de la AA a lo largo del tiempo, lo cual no ha sido nunca realizado anteriormente y es motivo de estudio en esta tesis.

El trabajo de la tesis finaliza con la presentación de un nuevo método para la predicción de la pronta terminación o el mantenimiento de episodios de FA paroxística. Este método se basa en el análisis de regularidad de características espectrales de la AA. Durante el diseño del método, se analizó la regularidad de las series numéricas de doce características espectrales diferentes. Para construir estas series fue obtenido previamente el espectrograma de la AA. La regularidad de las series se estimó mediante el índice de regularidad no lineal entropía muestral. La entropía muestral de seis de las características espectrales analizadas resultó ser estadísticamente relevante para la caracterización de la FA paroxística con $p<0.05$ en todos ellos. Este estudio fue complementado con un análisis multivariante de regularidad que lleva a cabo un estudio conjunto de las series de características espectrales y la AA en el dominio temporal. El análisis multivariante desvela la combinación de características que optimiza la predicción, de manera que el porcentaje de episodios correctamente clasificados alcanza el $100 \%$ para el grupo de aprendizaje y el $93.33 \%$ para el grupo de test. Por tanto, el método presentado puede ser utilizado con suficiente fiabilidad para predecir la terminación de la FA paroxística. 


\section{Resum}

Les arrítmies supraventriculars, en particular la fibri-lació auricular (FA), són les enfermetats cardíaques més comunment trobades en la pràctica clínica rutinària. La prevalència de la FA és inferior al 1\% en la població menor de 60 anys, però augmenta de manera significativa a partir dels 70 anys, apropant-se al 10\% en els majors de 80. El patiment d'un episodi de FA sostinguda, a més d'estar 1ligat a una major taxa de mortalitat, augmenta la probabilitat de sofrir tromboembolisme, infart de miocardi i accidents cerebrovasculars. Per una altra banda, els episodis de FA paroxística, aquella que finalitza de manera espontània, són els precursors de la FA sostinguda, la qual cosa suscita un alt interés entre la comunitat científica per conéixer els mecnanismes responsables de perpetuar o conduir a la finalització espontània dels episodis de FA.

L'anàlisi de l'ECG de superfície és la tècnica més estesa en la diagnosi mèdica de les patologies cardíaques. Per utilitzar l'ECG com a eina d'estudi de la FA, es necessita separar l'activitat auricular (AA) de la resta de senyals cardioelèctriques. En aquest sentit, les tècniques de Separació Cega de Fonts (BSS) són capaces de realitzar un anàlisi estadístic multiderivació amb l'objectiu de recuperar un conjunt de fonts cardioelèctriques independents, entre les quals es troba l'AA. A l'hora d'abordar un problema de BSS, es fa necessari considerar un model de mescla de les fonts el més ajustat possible a la realitat per poder desenvolupar algoritmes matemàtics que el resolguen. Un model viable és aquell que suposa mescles lineals. Dins d'aquest model de mescles lineals es pot a més fer la restricció de que aquestes siguen instatànies. Aquest model de mescla lineal instantània és l'utilitzat en l'Anàlisi de Components Independents (ICA). Un model de mescla alternatiu és el considerat pels algortimes BSS convolutius (CBSS), on es té en compte un procés de generació més realista de les derivacions de l'ECG amb contribucions retardades de fonts cardioelèctriques.

En aquesta tesi s'ha dut a terme per primera vegada un estudi de rendiment dels algoritmes CBSS aplicats a l'extracció de l'AA a partir de registres d'ECG. Per fer això, s'han comparat els algoritmes CBSS més rellevants amb l'algoritme instantani FastICA, l'efectivitat del qual està ja ampliament contrastada. Aquesta comparació permetrà saber quins algoritmes CBSS resulten ser útils per extraure l'AA dels registres d'ECG d'episodis de FA. Per un altre costat, els algoritmes 
CBSS presenten el problema de requerir un nombre mínim de senyals observades per a la seua adequada aplicació. Ací es presenta un nou algoritme per a l'extracció de l'AA, basat en el model convolutiu de mescla, que resol el problema de l'escassesa de derivacions que posseixen els registres Holter. L'alt grau de similitud entre l'AA original i l'AA estimada, mesurat pels diferents indicadors de rendiment utilitzats, demostra la idoneïtat del nou mètode per a l'extracció de l'AA a partir de registres Holter, a més de posar de relleu una major robustesa front al soroll del model convolutiu de mescla.

La causa més comú que explica l'aparició d'episodis de FA és el mecanisme de reentrada, que consisteix en l'existència de múltiples fronts de propagació que recorren el teixit auricular. Estudis recents han demostrat la relació existent entre el nombre d'entrades simultànies i l'organització de l'activitat elèctrica de les aurícules. Per altra banda, també són coneguts la progressiva deterioració de l'organització de l'activitat elèctrica auricular després del començament de la FA paroxística i l'increment de dita organització en els instants previs al retorn a ritme sinusal normal. El manteniment dels episodis de FA està a més relacionat amb la variabilitat de dispersió en freqüència de l'AA. Des d'un punt de vista clínic, resulta interessant avaluar el nivell d'organització elèctrica de les aurícules mitjançant indicadors de regularitat aplicats a l'AA extreta de l'ECG de superfície amb l'objectiu de predir l'evolució de la FA paroxística. Açò es pot dur a terme desde dues perspectives distintes. La primera forma de fer-ho seria aplicar l'estimació de regularitat a l'AA tal com l'obtenen els mètodes d'extracció. Una manera alternativa d'abordar el problema seria estudiar la regularitat de certes característiques espectrals de l'AA al llarg del temps, la qual cosa no ha estat mai realitzada amb anterioritat i és motiu d'estudi en aquesta tesi.

El treball de la tesi finalitza amb la presentació d'un nou mètode per a a predicció de la sobtada terminació o el manteniment d'episodis de FA paroxística. Aquest mètode es basa en l'anàlisi de regularitat de característiques espectrals de l'AA. Durant el disseny del mètode es va analitzar la regularitat de les sèries numèriques de dotze caracterísitiques espectrals diferents. Per construir aquestes sèries es va obtenir previament l'espectrograma de l'AA. La regularitat de les sèries va ser estimada amb l'index de regularitat no lineal entropia mostral. L'entropia mostral de sis de les característiques espectrals analitzades va resultar ser estadísticament relevant per a la caracterització de la FA paroxística amb $p<$ 0.05 per a tots ells. Aquest estudi va ser completat amb un anàlisi multivariant de regularitat que realitza un estudi conjunt de les sèries de característiques espectrals i de l'AA en el domini temporal. L'anàlisis multivariant desvela la combinació de paràmetres que optimitza la predicció, de manera que el percentatge d'episodis correctament classificats alcança el 100\% per al grup d'aprenentatge i el $93.33 \%$ per al grup de test. Per tant, el mètode presentat pot ser utilitzat amb suficient fiabilitat per a predir la terminació de la FA paroxística. 


\section{Agradecimientos}

Sirvan estas líneas para expresar mi gratitud a todas aquellas personas que han estado cerca de mí y me han dado su meritorio apoyo durante el desarrollo de esta tesis, pues todos ellos me han animado a lograr los objetivos perseguidos.

En primer lugar, quiero agradecer la inmensa ayuda que he recibido por parte de mi director de tesis José Joaquín Rieta, sin cuya supervisión y sus valiosos consejos, avalados por su amplia experiencia investigadora en el campo de la ingeniería biomédica, la consecución de este trabajo no hubiera sido posible. Es un enorme orgullo para mí haber podido trabajar con él tanto como docente como investigador en la Universidad Politécnica de Valencia. Mi paso por el Departamento de Ingeniería Electrónica ha marcado muy positivamente mi desarrollo profesional y me ha abierto puertas en el apasionante mundo de la investigación al conocer a personas como Juan Manuel Sanchis, a quien debo agradecer su estimable y desinteresada ayuda en los inicios de la tesis.

En segundo lugar, agradezco sinceramente a César Sánchez su calurosa acogida en Cuenca y el haberme dado la posibilidad de formar parte del Grupo de Innovación en Bioingeniería (GIBI) de la Universidad de Castilla-La Mancha, gracias a lo cual he podido trabajar codo con codo junto a Raúl Alcaraz, de cuya perseverancia y dedicación al trabajo investigador he aprendido tanto.

Deseo también expresar lo enormemente grato y enriquecedor que ha resultado poder colaborar e intercambiar ideas con el resto de componentes del GIBI. Los innumerables buenos momentos compartidos con ellos harán que mi pasada estancia en Cuenca sea inolvidable.

Finalmente, quiero dar las gracias a mis padres por su incondicional ayuda, y muy especialmente a mi esposa Silvia, que es quien más ha sufrido las contrariedades de este trabajo. Sin tu comprensión y sacrificio no hubiera sido posible superar los momentos de mayor dificultad que han surgido en el camino. Gran parte de esta tesis se ha escrito gracias a ti, por lo cual te estaré eternamente agradecido. 



\section{Contents}

1 Motivation, Hypotheses, and Objectives 1

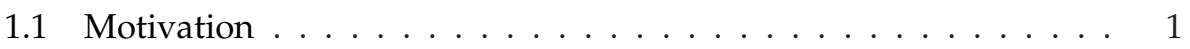

1.2 Initial Hypotheses . . . . . . . . . . . . . . . . 3

1.3 Objectives ........................... 4

1.4 Thesis Structure . . . . . . . . . . . . . . . . . 6

2 Basic Electrocardiography Concepts 9

2.1 Cardiovascular Physiology . . . . . . . . . . . . . . 10

2.1.1 Heart Anatomy . . . . . . . . . . . . . . . 10

2.1 .2 Cardiac Cycle . . . . . . . . . . . . . . . 11

2.2 Cardiac Conduction System . . . . . . . . . . . . . . . . . 11

2.3 The Electrocardiogram . . . . . . . . . . . . . . . 13

2.3.1 Leads of the Standard ECG . . . . . . . . . . . . . . . 13

2.3.2 Orthogonal Planes of the ECG . . . . . . . . . . . . . 17

2.3 .3 The Normal ECG . . . . . . . . . . . . . . . . . . . 18

2.3.4 Genesis of the ECG. The Heart Vector . . . . . . . . . . . . . 20

2.3.5 The ECG as a Clinical Tool for Cardiac Diagnosis . . . . . . 21

3 Atrial Fibrillation and its Linear Model 25 
3.1 Definition and Clinical Manifestation . . . . . . . . . . . . . . 26

3.2 Classification . . . . . . . . . . . . . . . . 27

3.3 Electrophysiological Mechanisms . . . . . . . . . . . . . . 28

3.4 Epidemiology . . . . . . . . . . . . . . . . . . . . . . 29

3.5 Atrial Fibrillation and Quality of Life . . . . . . . . . . . . 33

3.6 Bioelectric Model of Atrial Fibrillation . . . . . . . . . . . . . . 34

3.6.1 Potentials on the Body Surface . . . . . . . . . . . . . 34

3.6.2 Independence and Nongaussianity of Atrial and Ventricular Activities . . . . . . . . . . . . . . . . . . 40

4 Estimation Strategies of the Atrial Activity from the ECG 43

4.1 Time-domain Based Techniques . . . . . . . . . . . . . . . . . . . 44

4.2 Blind Source Separation . . . . . . . . . . . . . . . . 46

4.2.1 General Mixing Model . . . . . . . . . . . . . . . . . . 46

4.2.2 Instantaneous Linear Mixing Model . . . . . . . . . . . . 47

4.2.3 Convolutive Linear Mixing Model _ . . . . . . . . . . . 50

4.2.4 Global Matrix G . . . . . . . . . . . . . . . . . . . . 51

4.3 Applicability of BSS to Estimate the Atrial Activity . . . . . . . . . 53

4.3.1 Conditions of the Sources in BSS . . . . . . . . . . . 53

4.3.2 Separability and Identifiability $\ldots \ldots \ldots \ldots$

4.4 Separation Criteria for Convolutive BSS . . . . . . . . . 56

4.5 Convolutive BSS algorithms Under Test . . . . . . . . . . . . 57

$4.5 .1 \quad \operatorname{Infomax} \ldots \ldots \ldots \ldots \ldots \ldots$

4.5 .2 MBLMS ................... 58

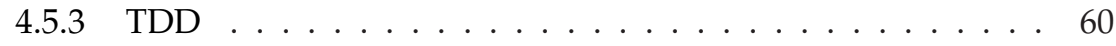

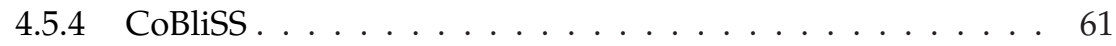

4.6 Atrial Activity Extraction from Holter Recordings . . . . . . . . 62

4.6.1 Inherent Problems . . . . . . . . . . . . . . . . . . . . 62 
4.6.2 Wavelet Transform Principles . . . . . . . . . . . . . . 63

4.6.3 A New Algorithm for Atrial Activity Estimation: Convolutive Multiband Blind Separation . . . . . . . . . . . . 66

5 Prediction of Paroxysmal Atrial Fibrillation Termination 71

5.1 Sample Entropy as a Regularity Estimator . . . . . . . . . . . . 72

5.2 Multi-Parametric Sample Entropy _ . . . . . . . . . . . . . . 74

5.2.1 Shared Steps . . . . . . . . . . . . . . . . . . . . . . . . 75

5.2.2 Univariate Analysis . . . . . . . . . . . . . . . . 76

5.2.3 Multivariate Analysis . . . . . . . . . . . . . . . . 77

5.2.4 Selection of the Optimal Time-Frequency Distribution . . . . 78

5.3 ECG Signal Quantization Effects . . . . . . . . . . . . . 79

5.3 .1 Introduction . . . . . . . . . . . . . . . 79

5.3 .2 Methodology . . . . . . . . . . . . . . 80

5.4 Poincaré Plots of Time-Frequency Parameters . . . . . . . . . . . 82

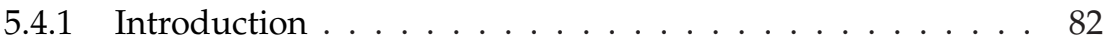

5.4 .2 Methodology . . . . . . . . . . . . . . . . . 82

6 Database and Performance Analysis $\quad 85$

6.1 Database Source and Signal Preprocessing . . . . . . . . . . . . . 86

6.2 Performance Analysis of CBSS Algorithms . . . . . . . . . . 86

6.2 .1 Testing Environments . . . . . . . . . . . . . . 86

6.2.2 Performance Indicators _. . . . . . . . . . . . . . . . 90

6.2.3 Identification of the AA source . . . . . . . . . . . . . . 94

6.3 Performance Analysis of CMBS . . . . . . . . . . . . . . 94

6.3.1 Testing environments $\ldots \ldots \ldots \ldots$

6.3.2 Performance Indicators . . . . . . . . . . . . . . . 96

6.4 Performance Analysis of AF Termination Prediction . . . . . . . . . 96

6.4.1 Testing Environment . . . . . . . . . . . . . . . . . . . 97 
6.4.2 Spectral Features in MPSE . . . . . . . . . . . . . . . . 97

6.4.3 Performance Indicators of Quantization Effects . . . . . . . . 98

$\begin{array}{lll}7 & \text { Results } & 101\end{array}$

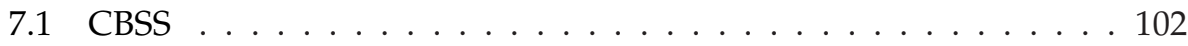

7.1 .1 First Environment . . . . . . . . . . . . . . . 102

7.1.2 Second Environment . . . . . . . . . . . . . . . . 113

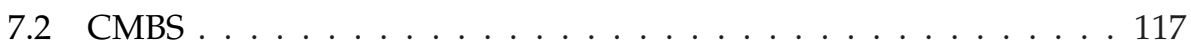

7.2.1 First Environment . . . . . . . . . . . . . . . . . 117

7.2.2 Second Environment . . . . . . . . . . . . . . . . . 122

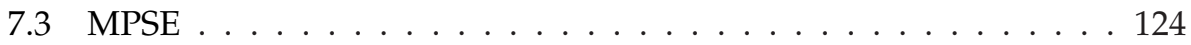

7.3.1 Selection of the Time-Frequency Distribution . . . . . . . . 124

7.3.2 Univariate Analysis . . . . . . . . . . . . . . . . . . 125

7.3.3 Multivariate Analysis . . . . . . . . . . . . . . . 130

7.4 Quantization Effects $\ldots \ldots \ldots \ldots \ldots \ldots$

7.5 Poincaré Plots . . . . . . . . . . . . . . . . . . . . . . 141

$\begin{array}{lll}8 & \text { Discussion } & 147\end{array}$

8.1 Estimation Strategies of AA . . . . . . . . . . . 147

8.2 Prediction of PAF Termination . . . . . . . . . . . . . . 151

9 Conclusions, Future Lines and Contributions 155

9.1 Conclusions . . . . . . . . . . . . . . . . . . . . 155

9.2 Future Lines . . . . . . . . . . . . . . . . . . . . . . 158

9.3 Contributions . . . . . . . . . . . . . . . . . . 159

9.3.1 Main Thesis Publications . . . . . . . . . . . . . . . . 159

9.3 .2 Collaborations . . . . . . . . . . . . . . 162

9.3 .3 Funding . . . . . . . . . . . . . . . . . . 165 
List of Tables 



\section{Chapter 1 \\ Motivation, Hypotheses, and Objectives}

\subsection{Motivation}

Atrial arrhythmias, in particular atrial fibrillation (AF), are the most commonly encountered arrhythmias in routine clinical practice [1]. Atrial fibrillation is a supraventricular tachyarrhythmia characterized by uncoordinated atrial activation with consequent deterioration of atrial mechanical function, so that atria contract in a disorganized manner when AF occurs [2]. The Franmingham Heart Study [3] reveals that the prevalence is less than $1 \%$ in people under 60 years old, but it increases significantly in those over 70, reaching 10\% for those over 80 . AF accounts for approximately one third of hospitalizations for cardiac rhythm disturbance, what explains the great interest of its study [1]. Furthermore, AF affects negatively to patient's quality of life [4] and contributes to worsening the prognosis of patients who have other comorbidities $[5,6,7]$.

The analysis of the electrocardiogram (ECG) is the most extended noninvasive technique in medical diagnosis of AF [8]. The exhaustive analysis of AF episodes requires to separate previously the atrial activity (AA) component from other bioelectric signals that contribute to the ECG formation [9]. Some of these signals are the ventricular activity (VA), the muscular activity, the noise and the artifacts introduced by the electrodes and the powerline interference [10]. The necessary operation of AA extraction is hindered by several difficulties. Firstly, AA presents in the ECG much lower amplitude - in some cases well under the noise levelthan its ventricular counterpart. Additionally, both phenomena possess spectral distributions notably overlapped, rendering linear filtering solutions unsuccessful [11]. To this extent, several techniques can be used in order to extract the AA from the ECG of AF episodes. On the one hand, time-domain techniques, like Average Beat Substraction (ABS) [12], try to obtain the AA by subtracting 
the average QRST complex. This techniques work on every ECG lead separately. On the other hand, Blind Source Separation (BSS) techniques [13, 14] use jointly the information included in every lead. BSS techniques basically consist of recovering a set of hidden source signals from a set of observed linear mixtures of the sources $[15,16]$. At present, the current BSS techniques are applied to AA extraction by considering the additional assumption of instantaneous mixing of sources [11]. Nonetheless, the instantaneous linear mixing model is proved not to be totally an exact description of the real mixing process of cardioelectric sources [17]. Therefore, the first motivation of this thesis is to find a new mixing model of cardioelectric sources more adjusted to the real mixing process so that a more accurate estimation of the AA from ECG recordings can be accomplished.

An accurate extraction of the AA from AF ECG recordings is the base for a subsequent precise and useful analysis which can help to the clinical management of the arrhythmia [9]. The management of patients with AF requires the knowledge of its pattern of presentation (paroxysmal, persistent, or permanent) and the underlying conditions in order to take decisions about restoration or maintenance of sinus rhythm, control of the ventricular rate, and antithrombotic therapy [8]. Paroxysmal AF (PAF) is often the precursor to sustained AF [18]. Given that sustained AF increases the likelihood of suffering myocardial infarctions and strokes [8], its accurate recognition by means of noninvasive techniques is of great interest to the regular clinical practice. The prediction of PAF maintenance can help to choose the appropriate intervention that may terminate the arrythmia and prevent its chronification, given that approximately $18 \%$ of patients who has intermittent AF degenerate into permanent AF four years after [19].

With reference to cost of AF management, the aim of the Euro Heart Survey was to estimate costs of admission and costs incurred on an annual basis by patients with AF in Greece, Italy, Poland, Spain and The Netherlands. It showed that inpatient care and interventional procedures were the main drivers of costs, accounting for more than $70 \%$ of total annual costs in all five countries. Therefore, the prediction of the spontaneous termination of PAF episodes could avoid unnecessary therapies and their associated clinical costs [20].

The PhysioNet/Computers in Cardiology Challenge 2004 [21] marked the start of continuous attempts to predict the early termination of PAF episodes by using the electrocardiogram (ECG). Several groups based their study on the atrial activity (AA) overall peak frequency plus additional spectral features as the main peak power [22] or time-frequency pattern [23]. Other groups tried to predict the evolution of PAF episodes by means of linear classifiers based on the main peak frequency and the mean RR interval [24, 25]. In [26] the spectral features fibrillation frequency, fibrillation amplitude and exponential decay are extracted from frequency-shifted and amplitude-scaled version of a log-spectral profile. The challenge is approached in [27] from a clinician's point of view by using features such as the $\mathrm{f}$-wave polarity, the $\mathrm{f}$-wave peak interval or the amplitude modulation of $\mathrm{AA}$, and a support vector machine is used as classifier. A recent publication extends the work carried out in [25] by including stepwise discriminant analysis 
and a greater number of spectral features [28].

The signal regularity analysis of ECG recordings is used in [29] as a means of predicting the evolution of PAF episodes. On the other hand, the time-frequency analysis is proved to be feasible for individualized noninvasive characterization of AF [30]. Time-frequency analysis is a powerful tool for unveiling the temporal variation of the atrial signal, whether such variation is spontaneous or due to an intervention [31]. Nevertheless, the relation between the regularity of AA signals and the evolution of PAF episodes is a matter that has never been explored. In fact, the likely connection between AA regularity in the time-frequency domain and the type of PAF episode constitutes the second motivation of this thesis.

\subsection{Initial Hypotheses}

BSS techniques have been successfully applied to the extraction of the AA from the ECG of AF episodes by assuming instantaneous and linear mixtures of the bioelectric signals in the human body [10]. Nevertheless, the assumption of instantaneous mixing of bioelectric sources involves an approximation error due to propagation effects [17]. Besides, the generation process of ECG leads implies the misalignment of lead fiducial points [32]. These two effects might be better explained by a mixing model different from the instantaneous one where relative delays among leads could be taken into account. In a new proposed mixing model it is assumed that the ECG consists of weighted and delayed contributions of different bioelectric signals. This new mixing model will be later introduced in section 4.2 as the convolutive linear mixing model. The first hypothesis of the thesis postulates that the accuracy of the estimated AA can be improved when the convolutive linear mixing model is used instead of the instantaneous linear mixing model, given that the convolutive model is a better approximation to the real mixing process of cardioelectric sources. A particular concern of this thesis is working with Holter ECG recordings because of their easy availability in clinical practice and the ability to record data for a long period of time. An extension of the hypothesis consists of assuming that the convolutive mixing model will be more useful than the instantaneous one for designing a new algorithm that optimizes the AA extraction from Holter ECG recordings.

When AF occurs, the electrical impulses in the atria degenerate from a normally organized pattern into rapid chaotic patterns because of uncoordinated atrial activation [8]. On the ECG, the consistent P-waves of a normal ECG are replaced by rapid oscillations of fibrillatory waves with varying morphology and timing [33], which is usually related to an irregular ventricular response [8]. A progressive deterioration of AA organization within the first three minutes after $\mathrm{AF}$ onset is proved in [34] and in [35]. In addition, slowing and increased organization of atrial activation is observed prior to AF termination [35, 36]. The changes of AA organization in PAF episodes is used in [29] to predict their evolution by using regularity estimators in time domain. On the other hand, the 
study of PAF episodes can be tackled by considering spectral features of the AA. Holm et al showed that the main peak frequency is a robust measure of intraatrial cycle length with the closest correlation found between lead V1 and right atrial electrograms [37]. With respect to regularity in the time-frequency domain, the combination of electrical and structural changes is associated with a significant loss of spatiotemporal organization, and the maintenance of AF is related to the structural remodeling and variability in frequency dispersion [38]. A lower variability of frequency in atrial electrograms is associated with termination of AF [39]. Moreover, frequency mapping has been used in [40] to identify localized sites of high frequency activity potentials responsible for the maintenance of AF. Nevertheless, a study of AA organization in the time-frequency domain by using regularity estimators has never been carried out. The second hypothesis of this thesis is that an accurate PAF termination prediction can be given by the application in the time-frequency domain of regularity estimators to the previously extracted AA.

\subsection{Objectives}

The hypothesis that the convolutive mixture is a better description than the instantaneous one for cardioelectric sources is an assumption that must be proved. In other words, the proposed convolutive mixing model as an enhanced explanation of real mixing of sources needs to be validated. A way to do this is to apply the most relevant convolutive BSS (CBSS) algorithms to the AA extraction from ECG recordings and to quantify their extraction performance. The subsequent comparison of AA extraction performance with FastICA, one BSS algorithm based on the instantaneous mixing model and which accuracy of results has already been proved [11], will provide an elucidation of the convolutive mixing model feasibility. On the other hand, the reduced number of leads in a Holter ECG makes unworkable the application of BSS techniques to this type of recordings because the number of leads must be at least the number of cardioelectric sources [14]. Although there exist other techniques for AA extraction that just require a unique lead, as Average Beat Subtraction (ABS) [12], this kind of techniques supplies an AA dependent on the selected lead. Only BSS techniques can offer an unified AA that takes into account the information from each lead [11]. Consequently, it must be found the means to increase the number of observations, i.e. leads, from a Holter ECG. This increase of leads can be carried out by wavelet decomposition of ECG leads into frequency subbands. The wavelet decomposition was first applied in [41] by considering the instantaneous mixing model. On the contrary, our interest is to obtain an unified AA from Holter ECG recordings by contemplating the convolutive mixture model. Therefore, two main objectives of this thesis can be already exposed. In the one hand, one main goal is to reveal which present CBSS algorithms are reliable to extract the AA from the ECG of AF episodes in order to assess the convolutive mixing model. In the other hand, a second goal is to improve the AA estimation with respect to the instantaneous 
BSS algorithms by designing a new extraction method based on the convolutive mixing model. The aim of this new method is to improve the performance of current extraction methods. This will be mainly centered to solve the singular problems of AA extraction from Holter ECG systems.

The reason for seeking an accurate AA extraction is its medical applicability, since it is accepted that noninvasive measurement of fibrillatory waves is useful for the treatment and management of patients $[9,42]$. Once the AA is obtained with enough accuracy from ECG recordings, it can be used for the characterization of PAF episodes by signal analysis. Recognizing the conditions under which PAF is likely to self-terminate might help to choose the most suitable intervention in affected individuals [21] and to refrain from applying unnecessary therapies [20]. The analysis proposed in this thesis entails studying the regularity of certain spectral features of the AA source in the time-frequency domain. In order to evaluate the regularity of AA spectral features, the nonlinear index Sample Entropy (SampEn) [43] was selected. This nonlinear index was chosen because of the chaotic activation of the atria in the diseased heart and the far-from linear process of electrical remodeling in AF [44, 45]. The SampEn is proved to be a useful regularity estimator for the study of electroencephalography signals and the characterization of neural pathologies [46]. The SampEn is also demonstrated to be a suitable regularity estimator for PAF characterization and was first used in time-domain to predict PAF evolution in [29]. Given the already commented AA frequency regularity changes prior to PAF termination, SampEn might be an accurate predictor in the time-frequency domain of PAF episodes evolution. The ratio of the area under the dominant frequency of AA and its harmonics to the total spectral power is used in [47] as a spectral index able to predict the AF termination. Nonetheless, in contrast to SampEn, this index cannot take into account the variability in time of spectral properties. A promising innovation of the present thesis is the application of SampEn to a group of direct and derived spectral features with the aim to predict the termination of PAF episodes with notable accuracy. The SampEn will be applied to spectral features extracted from time-frequency distributions so that the variable values of features along time can be considered. This results in the formulation of two additional objectives of this thesis. Firstly, the SampEn will be assessed as a suitable predictor of PAF termination in the time-frequency domain. Secondly, a new algorithm based on the regularity of spectral features will be designed for predicting the evolution of PAF episodes from Holter ECG recordings.

In sum, this thesis is focused on the accomplishment of the following explicit objectives:

- Assessment of the convolutive mixing model feasibility for cardioelectric sources by analyzing the AA extraction performance of the most relevant CBSS algorithms.

- Design of a new CBSS algorithm able to improve the AA extraction performance of current extraction algorithms and to solve the problem of lack of 
available leads from Holter ECG recordings.

- Assessment of the regularity estimator $\operatorname{SampEn}$ as an accurate predictor of PAF episodes termination when applied to series of spectral features.

- Design of a new algorithm based on the application of SampEn in the timefrequency domain that allows to predict the PAF episodes evolution from Holter ECG recordings.

\subsection{Thesis Structure}

This thesis consists of nine chapters where three main parts can be distinguished. Chapters 1, 2 and 3 constitute the first part of the thesis, where the subject of study is exposed along with the basic introductory notions and the necessary previous study of the state of the art. The second part or the thesis comprises chapters 4,5 and 6. The methods applied are introduced in chapters 4 and 5, while the description of the recordings database and the test environments is made in chapter 6 . Finally, the third part is composed by chapters 7,8 and 9 . Results of the thesis are presented in chapter 7 whilst the discussion on results and the final conclusions are reported in chapters 8 and 9, respectively. A more specific description of each subsequent chapter is made next:

- Chapter 2. In this chapter, the heart is described, firstly, as physiological organ, and secondly, as an electrical generator with its electrical conduction system. How the electrical activity of the heart is transmitted to the body surface and how this activity is registered by ECG recorders is described here as well. The features of a normal ECG are also introduced.

- Chapter 3. This chapter is focused on describing the AF disease. The social impact of this arrhythmia, its clinical manifestation, classification and electrophysiological mechanisms are treated matters here. On the other hand, the bioelectric model of AF that will be taken as a reference for the rest of the study is explained too.

- Chapter 4. This chapter is concerned with the AA estimation strategies from the ECG. A review of BSS fundamentals and the required conditions for its applicability are commented here. The several separation criteria that are the basis of different BSS algorithms is also a question that this chapter deals with. Besides, the most relevant CBSS algorithms, that will be used to assess the convolutive mixing model, are introduced. Finally, a new algorithm for AA estimation from Holter ECG recordings is presented.

- Chapter 5. In this chapter, the SampEn is put forward as a regularity estimator of spectral features. The design of a new method for PAF termination prediction based on the SampEn estimator is materialized here. This new 
method is optimized for convolutive mixture of sources and is able to increase the number of available observations from Holter ECG recordings. Moreover, the effects of Holter ECG signal quantization on the method applicability are considered. Finally, a complementary visual method is also proposed for PAF termination prediction.

- Chapter 6. In this chapter, the database of ECG recordings is described. Also, the environments for testing the performance of algorithms are explained. Furthermore, the necessary performance indicators for quantifying the suitability of the algorithms are defined in this chapter.

- Chapter 7. The results of the previously introduced methods are systematically presented in this chapter. The results are exposed for each testing environment described in chapter 6.

- Chapter 8. An analytic discussion on the results of chapter 7 is performed in this chapter in order to find a logical explanation of the obtained outcomes.

- Chapter 9. The concluding remarks on the accomplished work are exposed in this chapter. Furthermore, some possible future research lines are suggested. Finally, the main contributions in scientific symposia, conferences and SCI journals on biomedical engineering an cardiology are enumerated. 



\section{Chapter 2 \\ Basic Electrocardiography Concepts}

2.1 Cardiovascular Physiology . . . . . . . . . . . 10

2.1 .1 Heart Anatomy . . . . . . . . . . . . . . 10

2.1 .2 Cardiac Cycle .................... 11

2.2 Cardiac Conduction System . . . . . . . . . . . . 11

2.3 The Electrocardiogram . . . . . . . . . . . . . 13

2.3.1 Leads of the Standard ECG . . . . . . . . . . . . . . . 13

2.3.2 Orthogonal Planes of the ECG . . . . . . . . . . . 17

2.3 .3 The Normal ECG . . . . . . . . . . . . . . 18

2.3.4 Genesis of the ECG. The Heart Vector . . . . . . . . . . . 20

2.3.5 The ECG as a Clinical Tool for Cardiac Diagnosis . . . . 21

In this chapter, a brief description of the heart operation is made by considering the physiologic aspects and characteristic waveforms of the healthy heart. The electrocardiogram (ECG) is one of the most frequently used noninvasive body exploration that supplies a great amount of information on this vital organ. The main features of the electrocardiogram (ECG), as the ECG genesis or the electrocardiographic leads, will be reviewed here. The standard ECG leads and the axial reference systems will be explained and the different waves that form the normal ECG will be commented. Finally, the importance of the ECG as a tool for cardiac diagnosis will be highlighted. 


\subsection{Cardiovascular Physiology}

\subsubsection{Heart Anatomy}

The circulatory system is the network of elastic tubes that carries blood throughout the body. It includes the heart, lungs, arteries, arterioles (small arteries), and capillaries (very tiny blood vessels). These blood vessels carry oxygen and nutrient-rich blood to all parts of the body. The circulatory system also includes venules (small veins) and veins. These are the blood vessels that carry oxygenand nutrient-depleted blood back to the heart and lungs. The circulating blood brings oxygen and nutrients to all the body's organs and tissues, including the heart itself. It also picks up waste products from the body's cells. These waste products are removed as they are filtered through the kidneys, liver and lungs [48, 49].

The normal heart is a strong, muscular pump a little larger than a fist and its weight is about $250-300 \mathrm{~g}$. It pumps blood continuously through the circulatory system. The heart is located in the chest between the lungs behind the sternum and above the diaphragm. It is surrounded by the pericardium. Located above the heart are the great vessels: the superior and inferior vena cava, the pulmonary artery and vein, as well as the aorta. The aortic arch lies behind the heart. The location of the heart in the human body is depicted in Figure 2.1a [32, 50].

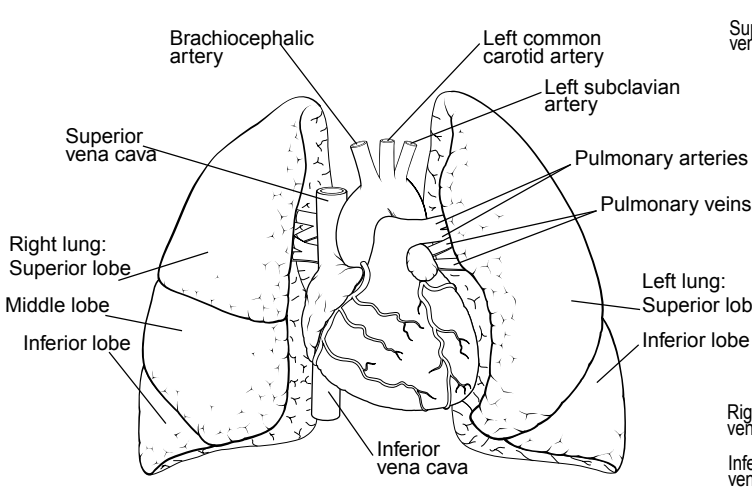

(a)

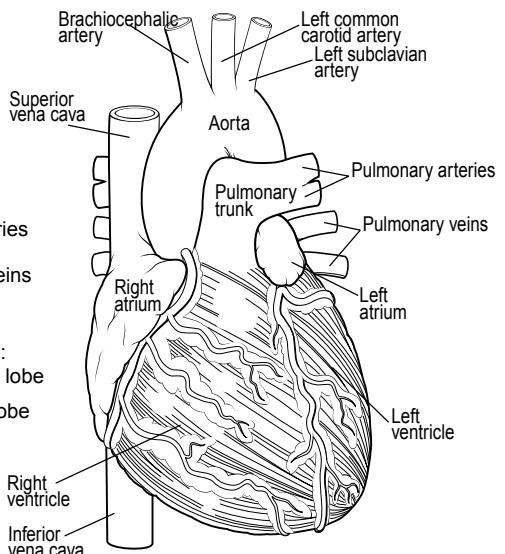

(b)

Figure 2.1. (a) Location of the heart in the human body and (b) structure of the heart.

Figure $2.1 \mathrm{~b}$ shows the structure of the heart. The heart has four chambers through which blood is pumped. The upper two are the right and left atria. The lower two are the right and left ventricles. There are four valves in the heart that open and close to let blood flow in only one direction when the heart beats. The tricuspid valve (also right atrioventricular valve) is between the right atrium and right ventricle. The pulmonary valve (also called right semilunar valve or 
pulmonic valve) is between the right ventricle and the pulmonary artery. The mitral valve (also left atrioventricular valve) is between the left atrium and left ventricle. The aortic valve (also called left semilunar valve) is between the left ventricle and the aorta. Under normal conditions, the valves let blood flow in just one direction. Blood flow occurs only when there's a difference in pressure across the valves that causes them to open [49].

\subsubsection{Cardiac Cycle}

Oxygen-poor blood returns to the heart after circulating through the body. It flows to the heart through veins and enters the right atrium. This chamber empties blood through the tricuspid valve into the right ventricle. The right ventricle pumps blood under low pressure through the pulmonary valve into the pulmonary artery. From there the blood goes to the lungs, where it gets fresh oxygen. Blood returns to the heart from the lungs via four pulmonary veins that enter the left atrium. From there it passes through the mitral valve and enters the left ventricle.

The left ventricle pumps the red, oxygen-rich blood through the aortic valve and into a large artery called the aorta. The aorta takes blood from the heart to the rest of the body.The left ventricle has a very thick muscular wall so that it can generate high pressures during contraction. Blood from the left ventricle is ejected across the aortic valve and into the aorta. While passing through the body, oxygen in the blood is distributed to the tissues. The cycle repeats as the blood flows back to the right atrium [49].

\subsection{Cardiac Conduction System}

The heart can be considered in a simplified description as a physiological organ that consists of two elements. The first element, already described, is the pump that impulses the blood through the body. The second element is the electric system that activates the pump, which includes an electric generator and a conduction system of electric pulses [32].

Figure 2.2 shows the conduction system of the heart. Located in the right atrium at the superior vena cava is the sinus node (sinoatrial or SA node) which consists of specialized muscle cells. The SA nodal cells are self-excitatory, pacemaker cells. They generate an action potential at the rate of about 70 per minute. The action potentials generated by the SA node spread throughout the atria primarily by cell-to-cell conduction. The conduction velocity of action potentials in the atrial muscle is about $0.5 \mathrm{~m} / \mathrm{sec}$. As the wave of action potentials depolarizes the atrial muscle, the cardiomyocytes contract by a process termed excitationcontraction coupling $[32,50]$. All cells of the conduction system are capable to generate action potential, but the intrinsic activation rate of the SA node is the 
greatest, what obliges the rest of cells to follow its rhythm. In case of a SA node disfunction, other cells would replace it so tha action potentials would be generated at lower rate $[50,51]$. Typical intrinsic activation rates of the conduction system are also indicated if Figure 2.2.

In the healthy heart, the only pathway available for action potentials to enter the ventricles is through a specialized region of cells (atrioventricular node, or AV node) located in the inferior-posterior region of the interatrial septum. The AV node is a highly specialized conducting tissue that slows the impulse conduction considerably (to about $0.05 \mathrm{~m} / \mathrm{sec}$ ) thereby allowing sufficient time for complete atrial depolarization and contraction (atrial systole) prior to ventricular depolarization and contraction. The impulses then enter the base of the ventricle at the Bundle of His and then follow the left and right bundle branches along the interventricular septum. These specialized fibers conduct the impulses at a very rapid velocity (about $2 \mathrm{~m} / \mathrm{sec}$ ). The bundle branches then divide into an extensive system of Purkinje fibers that conduct the impulses at high velocity (about $4 \mathrm{~m} / \mathrm{sec}$ ) throughout the ventricles. This results in depolarization of ventricular myocytes and ventricular contraction (ventricular systole). Figure 2.3 shows the different waveforms generated by the specialized cells heart conduction system in a normal ECG and the resultant body surface potential $[49,50]$.

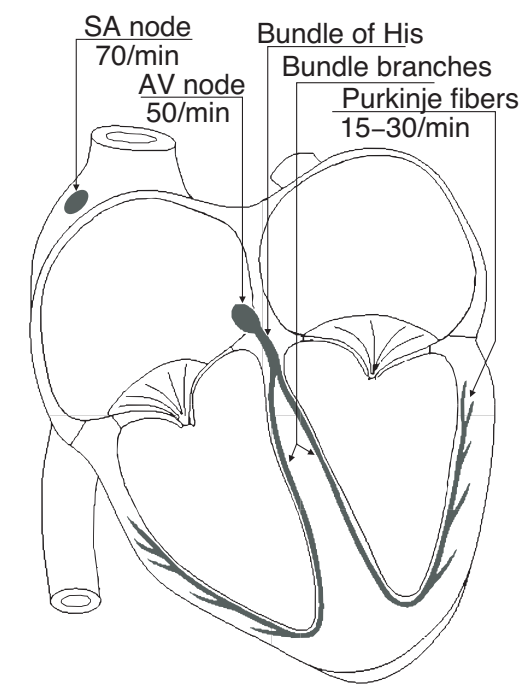

Figure 2.2. Parts of the heart conduction system and their activation rates. 


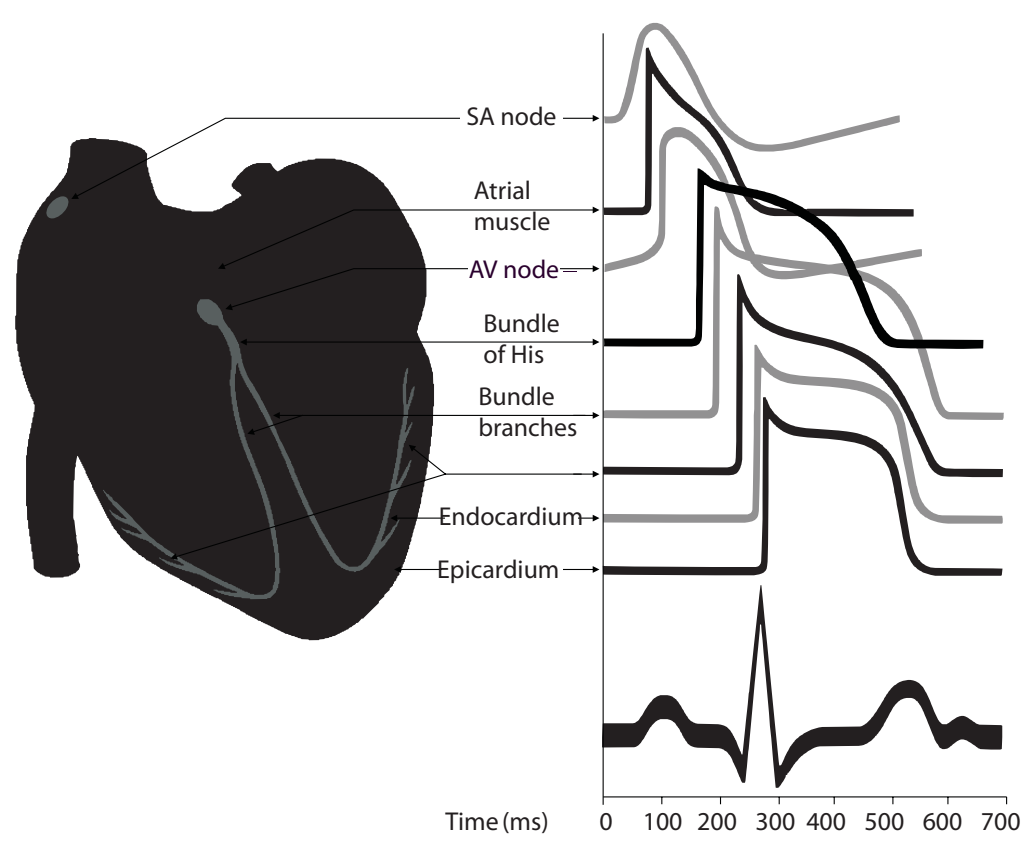

Figure 2.3. Different waveforms generated by the specialized cells heart conduction system in a normal ECG and the resultant body surface potential [50].

\subsection{The Electrocardiogram}

The electrocardiogram (ECG) is a recording of the electrical activity produced by the heart. The body acts as a giant conductor of electric currents. Any two points on the body may be paired by two electrodes to register the electrical activity of the heart. Every pair of these electrodes placed on the body surface constitutes a lead. These electrode leads are connected to a device that measures potential differences between selected electrodes, and the resulting tracing is called an electrocardiogram [51].

\subsubsection{Leads of the Standard ECG}

The standard ECG is composed by 12 beforehand fixed leads. There are two basic types of electrocardiogram (ECG) leads: bipolar and unipolar. Bipolar leads use a single positive and a single negative electrode between which electric potentials are measured. Unipolar leads have a single positive recording electrode and use a combination of electrodes to serve as a composite negative electrode [49]. 


\section{Einthoven Limb Leads}

In 1908 Willem Einthoven published a description of the first clinically important ECG measuring system. This measuring system consists of three bipolar ECG leads, namely leads I, II and III. He established the convention that lead I has the positive electrode on the left arm, and the negative electrode on the right arm, and therefore measures the potential difference between the two arms. In the Lead II configuration, the positive electrode is on the left leg and the negative electrode is on the right arm. Lead III has the positive electrode on the left leg and the negative electrode on the left arm [50]. That is:

$$
\begin{array}{cc}
\text { Lead I : } & V_{I}=\Phi_{L}-\Phi_{R} \\
\text { Lead II : } & V_{I I}=\Phi_{F}-\Phi_{R} \\
\text { Lead III : } & V_{I I I}=\Phi_{F}-\Phi_{L}
\end{array}
$$

where $V_{I}, V_{I I}$ and $V_{I I I}$ are the voltage of leads I, II and III, respectively. $\Phi_{L}, \Phi_{R}$ and $\Phi_{L}$ are the potential of the left arm, right arm, and left foot, respectively. These three bipolar limb leads roughly form an equilateral triangle (with the heart at the center) that is called Einthoven's triangle. Whether the limb leads are attached to the end of the limb (wrists and ankles) or at the origin of the limb (shoulder or upper thigh) makes no difference in the recording because the limb can simply be viewed as a long wire conductor originating from a point on the trunk of the body. These three leads constitute the so called 3-lead axial reference system [52], which is depicted in Figure 2.4 as unbroken lines.

Maximum positive ECG deflection will occurs in Lead I when a wave of depolarization travels parallel through the axis between the right and left arms. Similar statements can be made for leads II and III where the positive electrode is located on the left leg. For example, a wave of depolarization traveling towards the left leg will give a positive deflection in both leads II and III because the positive electrode for both leads is on the left leg. A maximal positive deflection will be obtained in lead II when the depolarization wave travels parallel to the axis between the right arm and left leg. Similarly, a maximal positive deflection will be obtained in lead III when the depolarization wave travels parallel to the axis between the left arm and left leg $[49,52]$.

\section{Goldberger Augmented Limb Leads}

In 1942 E. Goldberger observed that the recorded ECG signals could be be replaced with a new set of leads that are called augmented leads because of the augmentation of the signal [50]. These leads have a limb positive electrode that is referenced against the midpotential of the two remaining limb electrodes. The positive electrodes for these augmented leads are located on the left arm $\left(a V_{L}\right)$, the right arm $\left(a V_{R}\right)$, and the left leg $\left(a V_{F}\right)$. Thus the potentials of the augmented 
limb leads are given by:

$$
\begin{aligned}
& a V_{R}=\Phi_{R}-\frac{\Phi_{L}+\Phi_{F}}{2} \\
& a V_{L}=\Phi_{L}-\frac{\Phi_{R}+\Phi_{F}}{2} \\
& a V_{F}=\Phi_{F}-\frac{\Phi_{R}+\Phi_{L}}{2}
\end{aligned}
$$

The three augmented leads are depicted in Figure 2.4 as broken lines. Lead $a V_{L}$ is at an angle of 30 degrees with reference to the lead I. Lead $a V_{R}$ and $a V_{F}$ are at angles of 150 and -90 degrees, respectively. These three augmented leads, coupled with the three Einthoven limb leads, constitute the so called six-lead axial system. These six leads system records the electrical activity along a single plane, termed the frontal plane relative to the heart. Using the axial reference system and these six leads, it is rather simple to define the direction of an electric vector at any given instant in time. If a wave of depolarization is spreading from right to left along the $0^{\circ}$ axis, then Lead I will show the greatest positive amplitude. Likewise, if the direction of the electric vector for depolarization is directed downwards $\left(-90^{\circ}\right)$, then $a V_{F}$ will show the greatest positive deflection. If a wave of depolarization is moving from right to left at $-30^{\circ}$, then $a V_{R}$ will show the greatest negative deflection $[49,50]$.

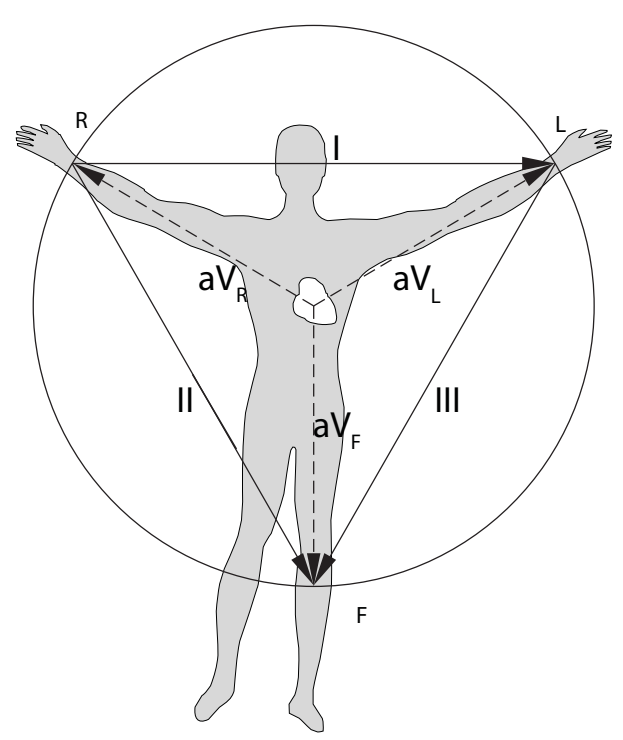

Figure 2.4. Leads I, II and II constitute the Einthoven's triangle or 3-lead axial reference system. The augmented leads, $a V_{R}, a V_{L}$ and $a V_{F}$, in conjunction with I, II and III constitute the 6-lead axial reference system[50] 


\section{Precordial Leads}

For measuring the potentials close to the heart, F. Wilson et al introduced the precordial leads, i.e. chest leads, in 1944. These are six positive electrodes placed on the surface of the chest over the heart in order to record electrical activity in a plane perpendicular to the frontal plane. The exact location of these six leads, namely $V_{1}, V_{2}, \cdots, V_{6}$, is indicated in Figure 2.5.

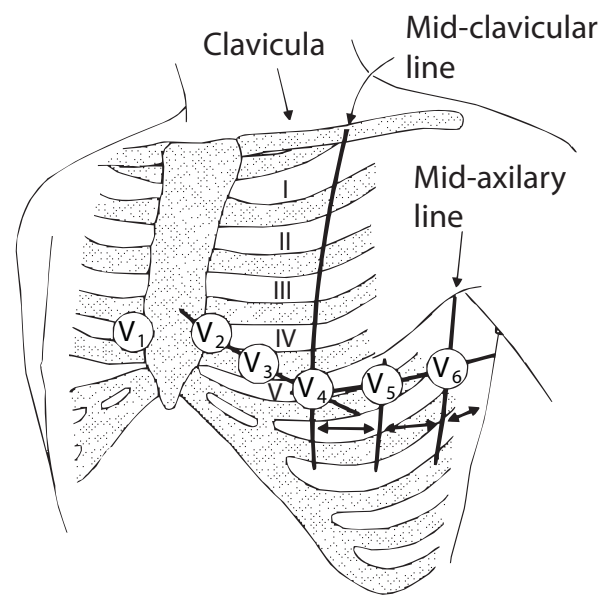

Figure 2.5. Location of the precordial leads on the chest.

Wilson investigated how electrocardiographic unipolar potentials could be defined. Ideally, those are measured with respect to a remote reference (infinity). Wilson suggested the use of the Wilson Central Terminal (WCT) as this reference. The CWT was initially formed by connecting a $5 \mathrm{~kW}$ resistor from each terminal of the limb leads to a common point. The potential of the WCT is calculated as [52]:

$$
\Phi_{C T}=\frac{\Phi_{R}+\Phi_{L}+\Phi_{F}}{3}
$$

Since the central terminal potential is the average of the Einthoven triangle vertexes potentials, and the addition of these potentials is approximately zero, the WCT can be therefore considered as a satisfactory reference. Although Wilson advocated $5 \mathrm{~kW}$ resistances and these are still widely used, at present the highinput impedance of the ECG amplifiers allows much higher resistances. A higher resistance increases the CMRR (common-mode rejection ratio) and diminishes the size of the artifact introduced by the electrode/skin resistance. The formation of the WCT is schematized in Figure 2.6 [52].

The rules of interpretation for precordial leads are the same as for the limb leads. For example, a wave of depolarization travelling towards a particular elec- 


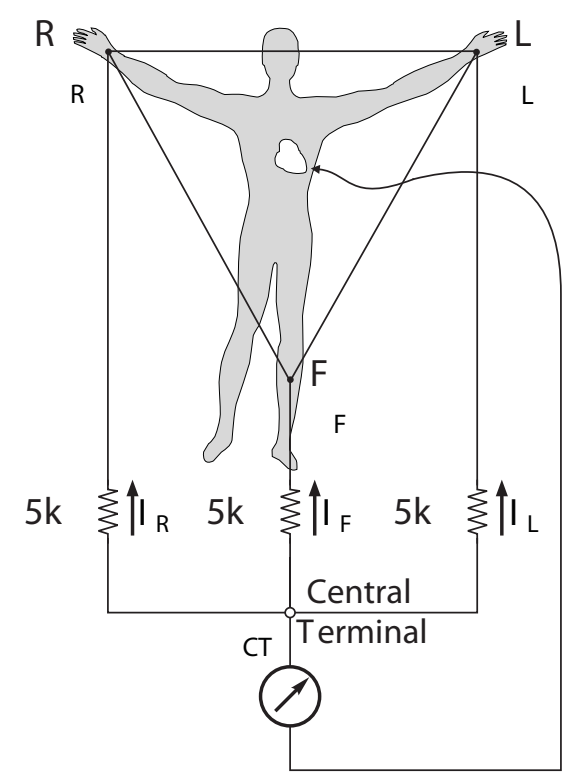

Figure 2.6. Formation of the Wilson Central Terminal

trode on the chest surface will elicit a positive deflection. In summary, the twelve ECG leads provide different views of the same electrical activity within the heart. Therefore, the waveform recorded will be different for each lead $[49,50]$.

\subsubsection{Orthogonal Planes of the ECG}

In principle, two of the limb leads (I, II, III) could reflect the frontal plane components, whereas one precordial lead could be chosen for the anterior-posterior component. The combination should be sufficient to describe completely the electric heart vector. (The lead V2 would be a very good precordial lead choice since it is directed closest to the $x$ axis. It is roughly orthogonal to the standard limb plane, which is close to the frontal plane.) To the extent that the cardiac source can be described as a dipole, the 12-lead ECG system could be thought to have three independent leads and nine redundant leads [52].

However, in fact, the precordial leads detect also nondipolar components, which have diagnostic significance because they are located close to the frontal part of the heart. Therefore, the 12-lead ECG system has eight truly independent and four redundant leads. The lead vectors for each lead based on an idealized (spherical) volume conductor are shown in Figure 2.7. These figures are assumed to apply in clinical electrocardiography [52].

The main reason for recording all 12 leads is that it enhances pattern recogni- 
tion. This combination of leads gives the clinician an opportunity to compare the projections of the resultant vectors in two orthogonal planes and at different angles. This is further facilitated when the polarity of the lead aVR can be changed; the lead -aVR is included in many ECG recorders [52].

In summary, for the approximation of cardiac electrical activity by a single fixed-location dipole, nine leads are redundant in the 12-lead system, as noted above. If we take into account the distributed character of cardiac sources and the effect of the thoracic surface and internal inhomogeneities, we can consider only the four of the six limb leads as truly redundant [52].

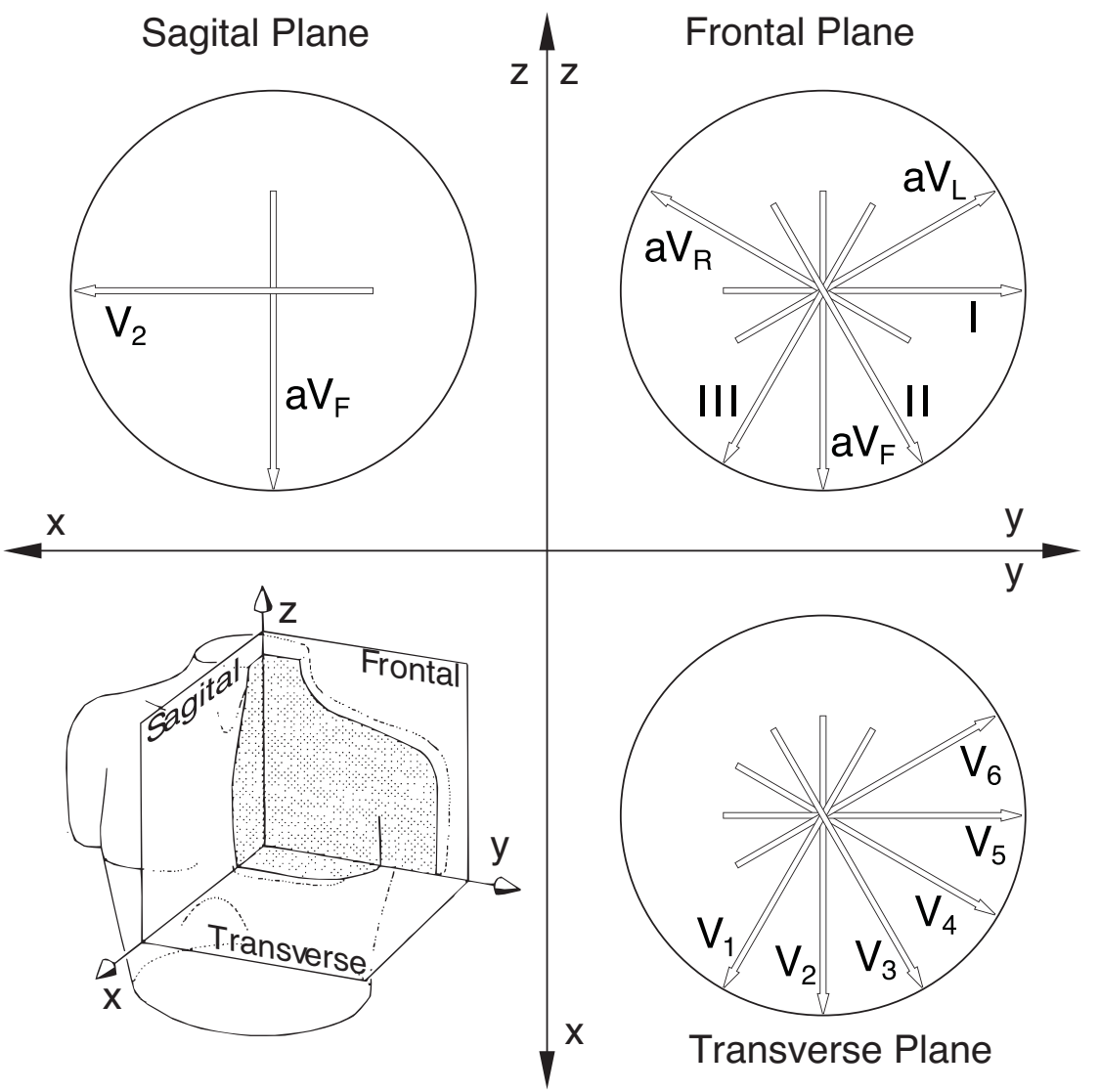

Figure 2.7. Projection of the 12-lead ECG system in three orthogonal planes: frontal, transverse and sagital [50].

\subsubsection{The Normal ECG}

The tracing recorded from the electrical activity of the heart forms a series of waves and complexes that have been arbitrarily labelled, in alphabetical order, 
the $\mathrm{P}$ wave, the QRS complex, the $\mathrm{T}$ wave and the $\mathrm{U}$ wave $[32,49]$. The normal ECG at lead I is depicted in Figure 2.8. Depolarization of the atria produces the P wave; depolarization of the ventricles produces the QRS complex. Repolarization of the ventricles causes the $T$ wave. The significance of the $U$ wave is uncertain, but it may be due to repolarization of the Purkinje system. The PR interval extends from the beginning of the $\mathrm{P}$ wave (the beginning of atrial depolarization) to the onset of the QRS complex (the beginning of ventricular depolarization) [52].

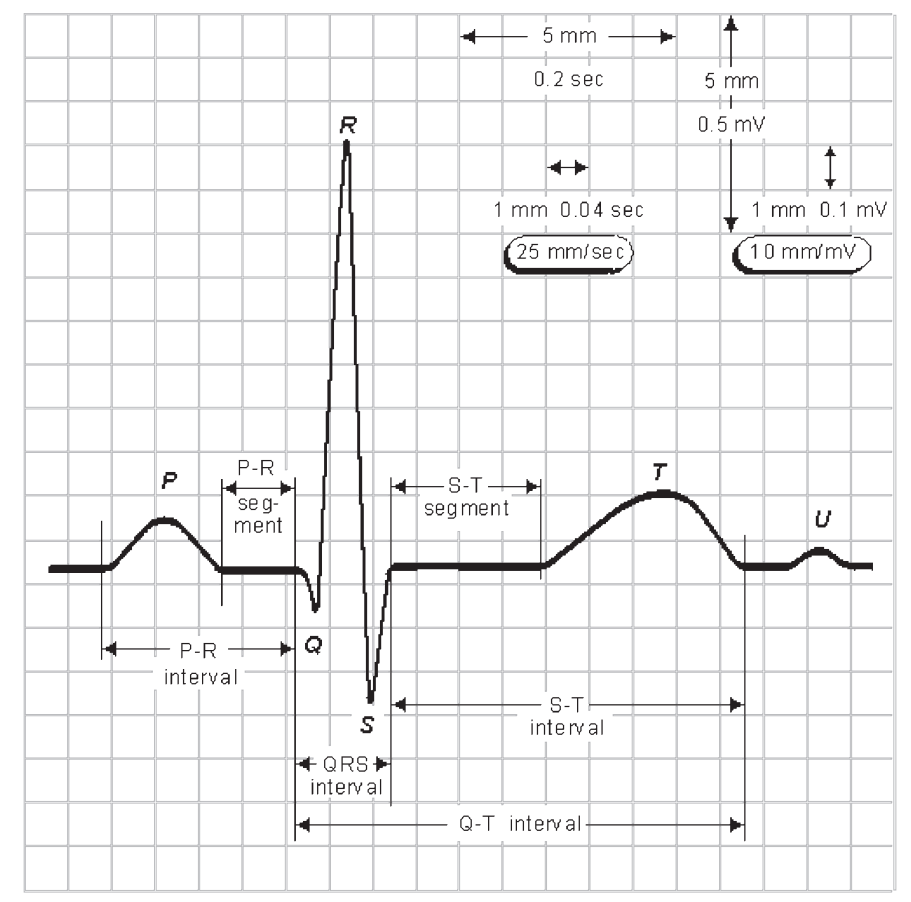

Figure 2.8. Waves of the normal ECG at lead I [50].

The normal ECG is characterized by [53]:

- P wave:

- Positive in I,II, $a V_{F}, V_{3-6}$

- Negative in $a V_{R}$

- Positive, negative or biphasic in III, $a V_{L}$ and $V_{1-2}$

- Maximum duration: 0.11 seconds.

- Heart Rate (HR): 60 to 100 beats per minute in rest condition.

- PR segment: isoelectric and maximum duration between 0.12 and 0.20 seconds. 
- QRS: duration between 0.06 and 0.12 seconds

- T wave: same direction as QRS, asymmetric morphology, at least the tenth part of R high

- ST segment: must be isoelectric, although deviations between 0.05 and 0.2 $\mathrm{mV}$ may be normal in precordial leads.

- U wave: occurs after the T wave and has the same direction, but frequently it is not registered.

\subsubsection{Genesis of the ECG. The Heart Vector}

The electrical activity of the heart can be modelled by a three-dimensional and time-variable dipole $\overrightarrow{\mathbf{H}}(t)$, which is the resultant heart dipole moment of the heart, and which is called the heart vector (HV) [54]. The current generators from a single heart cell can be considered dipoles. At any time, there will be a wave front of dipoles that can be treated mathematically using solid angles. When there are multiple dipoles, the resultant potential can be given by the spatial integral over the surface of the dipole front [55]. While the HV is a simplified representation of the electrical activity of the heart, this construct has been proven to be extremely useful, since models of the heart based on the HV provide an excellent simulation of the body surface ECG [56]. The measurement and display of the HV is called vectorcardiography. The three-dimensional graph plotted by the HV dipoled is referred as the vectorcardiogram.

The potential measured in one lead at any time can be expressed as the scalar product of two vectors [51]:

$$
u_{a b}(t)=\overrightarrow{\mathbf{H}}(t) \cdot \vec{l}_{a b}(t)
$$

where

$$
u_{a b}(t)=u_{a}(t)-u_{b}(t)
$$

is the bipolar potential, $\overrightarrow{\mathbf{H}}$ is the $\mathrm{HV}$, and $\vec{l}_{a b}$ is the lead vector from point $a$ to point $b$. The lead vector is expressed in terms of resistance per length unit, and it contains information on the geometry of the lead and on the resistivity distribution along the human body. It depends on the location of the source, the location of the observation point, the reference point, and the shape and conductivity of the torso and its internal structures [56]. The lead vector is a three-dimensional transfer coefficient which describes how the HV influences the potential measured in a particular lead $[32,50]$. 
The genesis of the ECG can be regarded as the HV projection on every lead. The direction and the magnitude of the HV change during the different stages of heart depolarisation and repolarisation. This is shown in Figures 2.9 and 2.10, where the HV is projected on leads I, II and III at different moments of the heart cycle. The rest of leads are also generated in the same way as a projection of the $\mathrm{HV}$ on the sagittal, frontal and transverse planes [32]. In a typical vectorcardiogram, we can distinguish three main loops:

- P loop, which is related to the depolarization of the atrial tissue during the atrial systole.

- QRS loop, which is related to the depolarization of the myocardium during the ventricular systole.

- $\mathrm{T}$ loop, which is related to the repolarization of the the myocardium during the ventricular diastole.

More realistic heart models divide the heart into a number of regions, and assign a lumped dipole $\overrightarrow{\mathbf{H}}_{i}(t)$ to the centroid of each region. In this case the cardiac electrical sources are represented by a finite number of lumped current dipoles. Such a source is a multiple dipole source [56]. The surface potential is then given by:

$$
u_{a b}(t)=\sum_{i=1}^{n} \overrightarrow{\mathbf{H}}_{i}(t) \cdot \vec{l}_{a b_{i}}(t)
$$

The presence of multiple electrical sources will be a basis, in this thesis, for the application of Blind Source Separation (BSS) to separate the Atrial Activity (AA) from other bioelectric sources. We will discuss this in depth in subsequent chapters.

\subsubsection{The ECG as a Clinical Tool for Cardiac Diagnosis}

There are two aspects to interpretation of the ECG. One is concerned with morphology of the waves and complexes, which make up a complete cardiac cycle. The other is concerned with timing of events and variations in patterns observed over many beats. Any change in cardiac electrical activity or in the volume conductor may be reflected in the ECG and provide diagnostic information. Cardiac electrical activity involves the shape of the action potential impulse and its propagation through the heart. This electrical activity determines the bioelectric sources. Volume conductor changes are often extracardiac and result in ECG changes. Included, for example, are conditions such as lung emphysema, and pericardial effusion where fluid forms around the heart. Diagnostic changes in the ECG of cardiac origin may be categorized as follows [56]. 

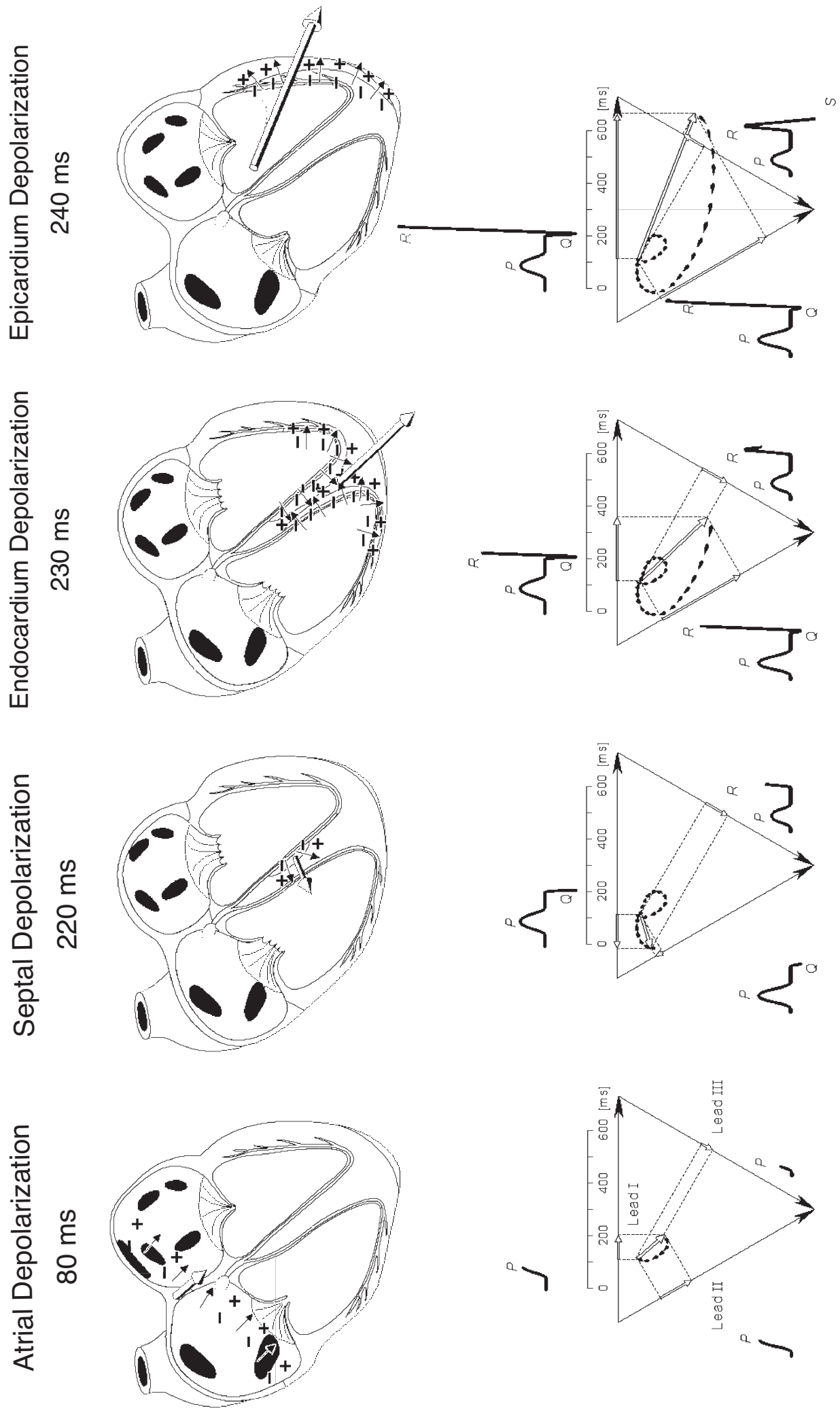

Figure 2.9. The generation of the ECG signal in the Einthoven limb leads [50]. 

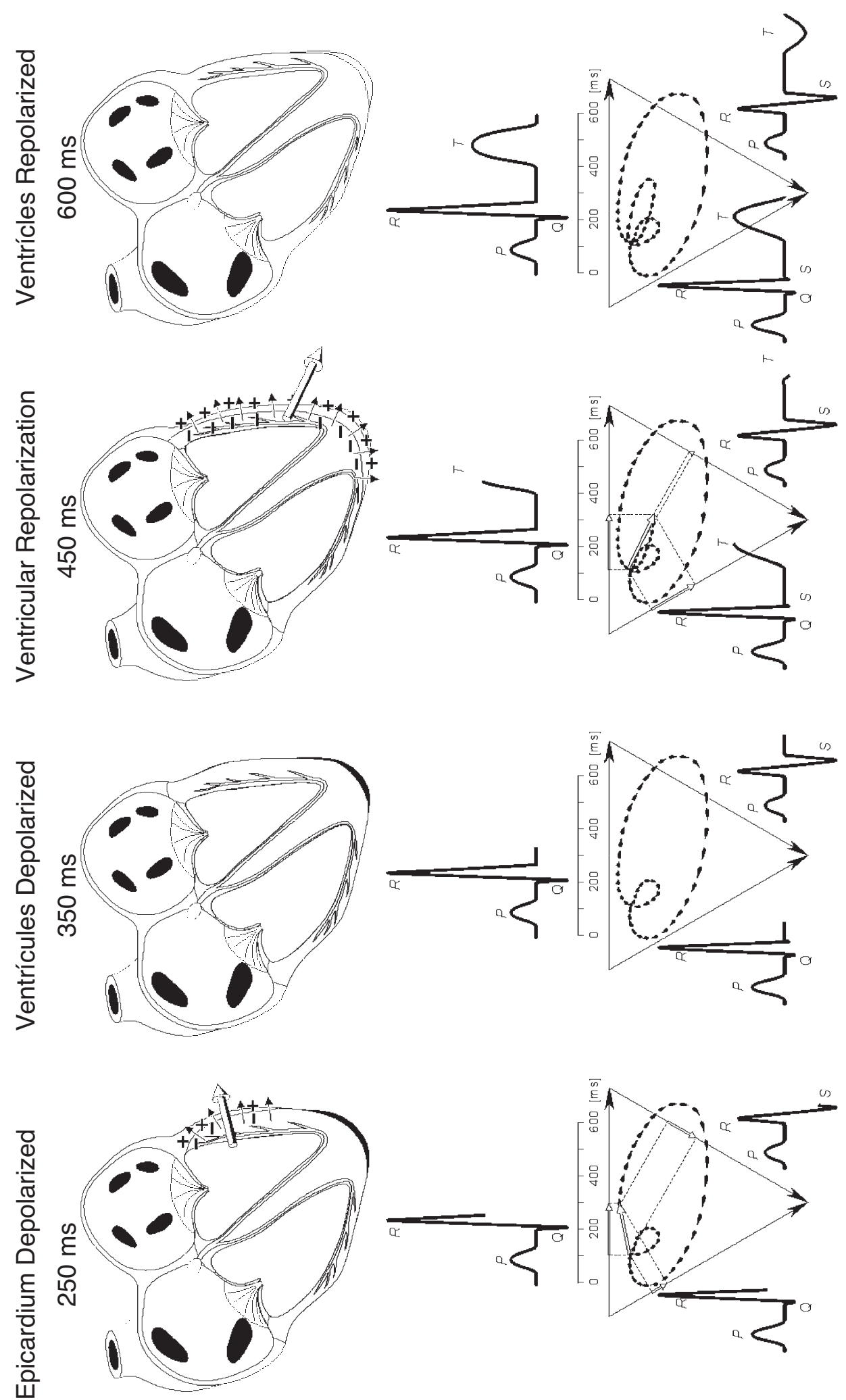

Figure 2.10. The generation of the ECG signal in the Einthoven limb leads (continued) [50]. 
- Injury: Injury to regions of the heart as a result of ischemia due to narrowing or occlusion of coronary arteries supplying the heart leads to changes in cellular action potentials and in the propagation of the impulse. The injury may be transient and reversible as during exercise or the early phases of myocardial infarction, or may become permanent after several hours. An example of the type of change observed in the ECG is a shift away from the baseline in the level of the ST segment which is the part of the waveform between the end of QRS and the beginning of T.

- Enlargement: Changes in the shape or position of the heart may result in ECG changes of diagnostic import. Of particular importance is enlargement or hypertrophy of one or more chambers of the heart. The atrial or ventricular cavity may enlarge, or the muscle wall may thicken.

- Conduction defects: Disease of any of the structures comprising the specialized conduction system, or presence of abnormal (accessory) pathways, may result in an alteration of the spread of excitation and the time course of recovery. Typical changes include slowed conduction and block of the impulse.

- Rhythm and rate: Information about heart rate is generally easily extracted from the ECG. Of immediate interest is whether the rate is too slow (bradycardia) or too fast (tachycardia), whether the ventricular rate is the same as the atrial rate, whether or not the rate is irregular, whether the disturbance is primarily atrial or ventricular. Disease of any portion of the heart can result in a disruption of its rhythm. The classification of cardiac arrhythmias and dysrhythmias involves more than 40 descriptors.

- Ionic effects: The cardiac action potential depends on the extracellular concentration of such ions as potassium and calcium, in addition to the presence of certain cardiotropic drugs such as digitalis. Abnormal levels may alter electrical activity and be manifest as changes in the ECG. 


\title{
Chapter 3 \\ Atrial Fibrillation and its Linear Model
}

\author{
3.1 Definition and Clinical Manifestation .......... 26

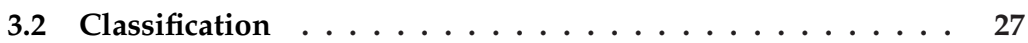 \\ 3.3 Electrophysiological Mechanisms . . . . . . . . . 28 \\ 3.4 Epidemiology . . . . . . . . . . . . . . . . 29 \\ 3.5 Atrial Fibrillation and Quality of Life . . . . . . . . . 33 \\ 3.6 Bioelectric Model of Atrial Fibrillation . . . . . . . . . 34 \\ 3.6.1 Potentials on the Body Surface . . . . . . . . . . . . 34 \\ 3.6.2 Independence and Nongaussianity of Atrial and Ven- \\ tricular Activities . . . . . . . . . . . . . . 40
}

After introducing the basic heart operation and the essential concepts on electrocardiography, a description of the atrial fibrillation will be made from a clinical point of view. Firstly, the definition of this arrhythmia and its clinical manifestation will be introduced. Secondly, the most extended classification of the atrial fibrillation will be established and the electrophysiological mechanisms involved in the occurrence of atrial fibrillation will be explained. Thirdly, the atrial fibrillation social impact will be portrayed by presenting the incidence, prevalence and effects on quality of life. Finally, the bioelectric model of atrial fibrillation used in the thesis, that validates the application of algorithms introduced in subsequent chapters, will be described. 


\subsection{Definition and Clinical Manifestation}

Atrial Fibrillation (AF) is the most commonly encountered arrhythmia in routine clinical practice. AF is a supraventricular tachyarrhythmia characterized by uncoordinated atrial activation with consequent deterioration of the atrial mechanical function. When AF occurs, the atria contract in a disorganized manner [8].

AF is capable of inducing electrophysiologic changes that promote further AF [57]. These include electrical, contractile and structural changes to the atria that have collectively become known as atrial remodelling. In the electrical remodelling process, the membrane channel responsible for calcium entry becomes less active. Eventually, the production of that channel is downregulated. These changes reduce the action potential duration and provoke the AF perpetuation [58].

On the other hand, studies conducted on animals and humans have demonstrated AF-related reductions in atrial contractile function and suggested that abnormal calcium handling at high rates may be responsible for remodelling [59, 60]. The presence of atrial fibrillation confers an alteration in hemodynamic state via two mechanisms: the loss of atrial systole and the irregular, fast ventricular response. Atrial fibrillation leads to a fall in stroke volume, varying from about $10 \%$ in normal individuals to over a third in the elderly. The reduction in stroke volume in atrial fibrillation is most marked at higher heart rates due to reduced left ventricular filling time [61].

AF is often related to increased atrial size, allowing a critical mass of anatomical substrate for the persistence of the disorganized electrical waveforms. Many conditions predisposing to AF are known to have effects on the left atrium, which are likely to be contributory to the initiation and maintenance of AF [61]. In addition, patchy areas of fibrotic change have been described on histological examination of atrial tissue from patients with AF [62]. There exist an association between atrial fibrosis and AF, although determining the casual importance of tissue fibrosis in AF occurrence and persistence remains an important challenge [63].

On the ECG, atrial fibrillation is described by the substitution of consistent $\mathrm{P}$-waves by rapid oscillations of fibrillatory waves ( $\mathrm{f}$-waves) that vary in size and shape and which rate typically ranges from 350 to 600 beats per minute [8]. The amplitude of $\mathrm{f}$-waves in AF is lower when compared to the amplitude of $\mathrm{P}$-waves during sinus rhythm [64]. Atrial fibrillation is also characterized by frequent irregular and rapid ventricular response [8]. The ECG provides a noninvasive way to study AF mechanisms. Analysis can be done through direct observation of the original signal or through methods used to obtain and analyze atrial activity (AA). The low amplitude of the AA in the surface ECG presents a challenge in analyzing f-waves characteristics [33]. The ventricular response to AF can vary with the electrophysiological properties of the AV node and the level of vagal and sympathetic tone [8]. Although the irregular ventricular response may be a clue for the presence of AF, this is a consequence of the arrhythmia and is not necessarily present [65]. Differences between normal ECG and atrial fibrillation ECG 
can be observed in Figure 3.1.

(a)

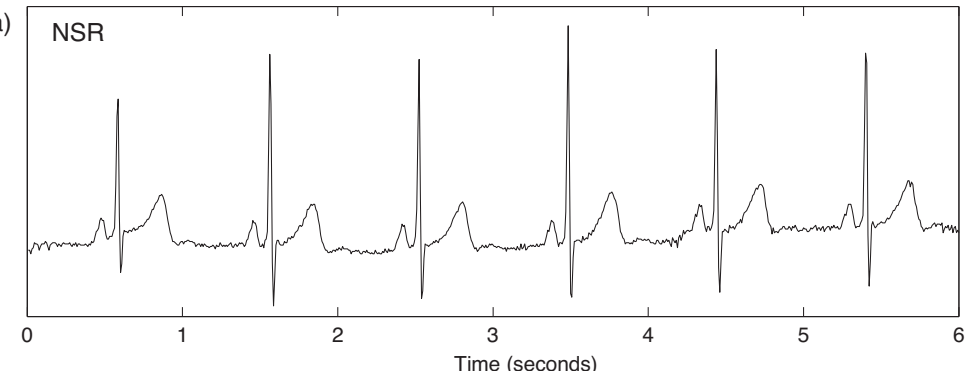

(b)

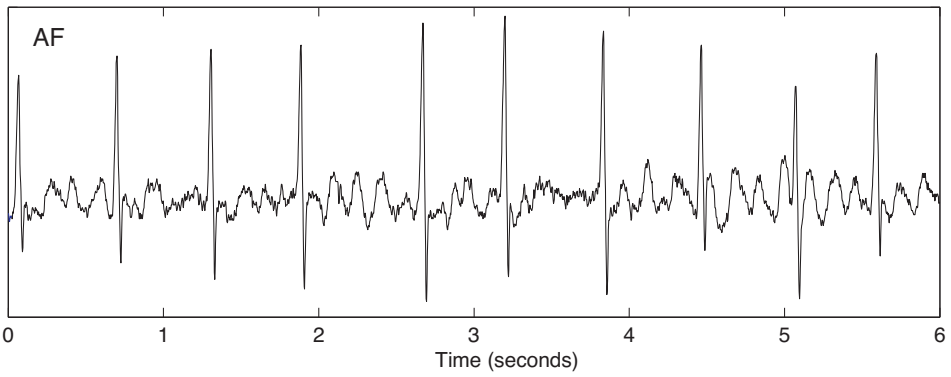

Figure 3.1. ECG of (a) Normal Sinus Rhythm (NSR) and (b) AF episode. In AF, the P waves are substituted by rapid oscillations of fibrillatory waves and the ventricular response becomes irregular.

\subsection{Classification}

In a general and summarized way, atrial fibrillation can be classified, according to the recommendations of the American College of Cardiology, as follows [8]:

- First detected: No other atrial fibrillation episodes were observed before in the patient.

- Recurrent: When two or more atrial fibrillation episodes are observed in the same patient.

- Paroxysmal: The arrythmia terminates spontanously. Atrial fibrillation episodes last less than seven days, and typically less than 48 hours.

- Persistent: At the contrary that in the previous case, persistent atrial fibrillation is sustained if no pharmacological therapy or electric cardioversion is done. Persistent AF may be either the first presentation of the arrhythmia or the culmination of recurrent episodes of paroxysmal AF. It usually lasts more than 7 days. 
- Permanent: Pharmacological therapy and electric cardioversion attempts failed to revert the heart to sinusal rhythm. It is the chronic state of AF and can last for years.

In figure 3.2 it is shown the relations between the different types of AF. Persistent AF may be either the first presentation of the arrhythmia or the culmination of recurrent episodes of paroxysmal atrial fibrillation.

Finally, the term lone atrial fibrillation is defined as AF in the absence of clinical or echocardiographic findings of cardiopulmonary disease. It generally applies to individuals under 60 years old who do not present any clinical evidence of cardiovascular pathology.

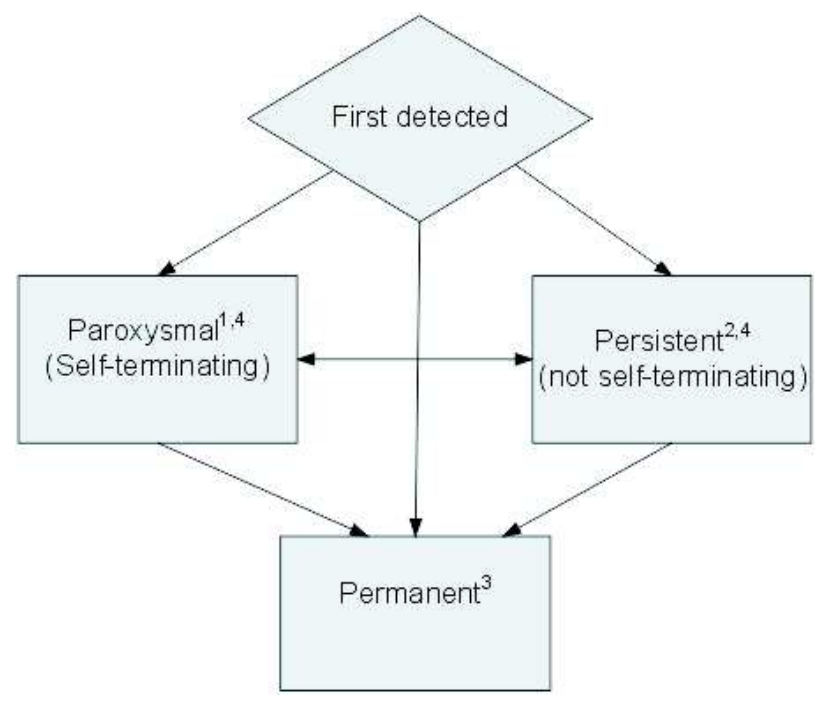

Figure 3.2. Patterns of atrial fibrillation. (1) episodes that generally last less than or equal to 7 days (most less than 24 h; (2) usually more than 7 days; (3) cardioversion failed or not attempted; and (4) either paroxysmal or persistent AF may be recurrent [8].

\subsection{Electrophysiological Mechanisms}

Theories of the mechanism of AF involve two main processes[8]: enhanced automaticity in one or several rapidly depolarizing foci (see Figure 3.3.a) and reentry involving one or more circuits (see Figure 3.3.b). In the first of the mechanisms, rapidly firing atrial foci, located in one or several of the superior pulmonary veins, can initiate atrial fibrillation in susceptible patients. Foci also occur in the right atrium and infrequently in the superior vena cava or coronary sinus. The focal origin of AF is supported by experimental models and appears to be more important in patients with paroxysmal AF than in those with persistent AF [8]. Nonetheless, the most extended and accepted theory is based on 
the reentry mechanism, which considers the existence of multiple wave propagation fronts. The multiple wavelet hypothesis as the mechanism of reentrant atrial fibrillation was advanced by Moe et al [66], who proposed that fractionation of the wave fronts as they propagate through the atria results in self-perpetuating "daughter wavelets." The number of wavelets present at any time depends on the refractory period, mass, and conduction velocity in different parts of the atria [8].

Reentry occurs when an impulse travels around an abnormal circuit repetitively. Let us consider two distinct areas of tissue (Figure 3.4), where area A is excited by a depolarizing wavefront. Once excited, cells in area A cannot be excited again until their cell membranes have repolarized and the cells have recovered; the depolarizing wavefront has left the cells in its wake refractory to further stimulus. A premature stimulus activating area B cannot excite area A if it occurs when the intervening tissue is still refractory. However, if that depolarizing wavefront travels to area A by an alternate route, allowing sufficient time for tissue in area A to recover, then area A may be re-excited. Under the right circumstances, areas A and B can then re-excite each other, which leads to sustained reentry [58].

There exist two different types of reentries: anatomic and functional reentries. In anatomic reentry, the boundaries of the circuit are physical cardiac structures. Alternatively, variations in the electrophysiologic properties of contiguous tissues, not anatomic obstacles, may serve as the boundaries of a functional reentry circuit. Functional reentry appears to be the most important mechanism in AF [58]. The likelihood that AF will terminate, either spontaneously or as a result of a medical intervention, is inversely related to the duration of the episode $[57,58]$. Recent studies have proved that, when an AF episode is near to its termination, the number of reentries decreases so that simpler waveforms are generated on the atrial tissue $[36,39,67]$. Consequently, $\mathrm{f}$-waves evolve to Pwaves while the AA becomes slightly more organized before AF termination [68].

\subsection{Epidemiology}

Atrial fibrillation is the most common arrhythmia encountered in clinical practice, accounting for approximately one third of hospitalizations for cardiac rhythm disturbance. It has been estimated that 2.2 million americans have paroxysmal or persistent atrial fibrillation[8]. The prevalence of AF that comes out from the Framingham Heart Study and the Cardiovascular Heart Study (CHS) is presented in Figure 3.5. The prevalence of atrial fibrillation is estimated at $0.4 \%$ of the general population, increasing with age. Atrial fibrillation is uncommon in childhood except after cardiac surgery. AF occurs in fewer than $1 \%$ of people under 60 years old but in more than $6 \%$ of those older than 80 years. The age-adjusted prevalence is higher in men than in women. Based on limited data, blacks have less than half the age-adjusted risk of developing atrial fibrillation that is seen in whites. The frequency of lone atrial fibrillation was less than $12 \%$ of all cases of 


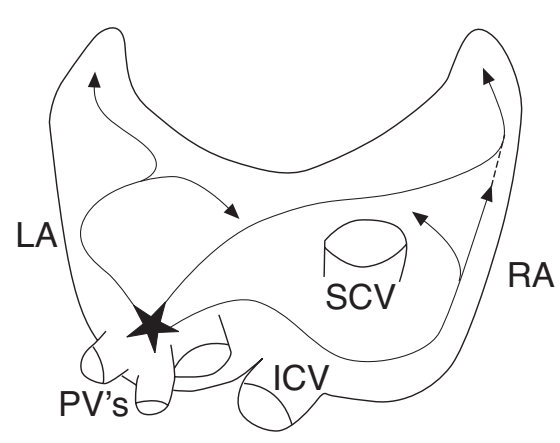

(a)

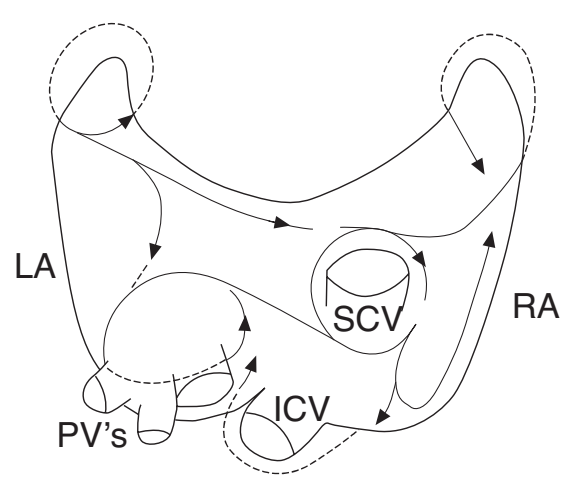

(b)

Figure 3.3. Principal electrophysiological mechanisms of atrial fibrillation. (a) Focal activation. The initiating focus (indicated by the asterisk) often lies within the region of the pulmonary veins. The resulting wavelets represent fibrillatory conduction, as in multiple-wavelet reentry. (b) Multiple-wavelet reentry. Wavelets (indicated by arrows) randomly reenter tissue previously activated by them or by another wavelet. The routes the wavelets travel vary. LA indicates left atrium; PV, pulmonary vein; ICV, inferior vena cava; SCV, superior vena cava; and RA, right atrium [1]

atrial fibrillation in some series but over 30\% in others. The prevalence of atrial fibrillation increases with the severity of congestive heart failure (HF) or valvular heart disease[8]. In the year 2000, roughly 2.25 million Americans had AF. By the year 2010, that number will go up to 2.66 million, which already is a third of a million increase, and it is projected to go up continuously and predicted to peak at the year 2050 at 5.5 million patients, what emerges from the ATRIA study (the AnTicoagulation and Risk Factors In Atrial Fibrillation) [69].

The incidence of AF emerged from the Framingham and CHS studies is show in 3.6. The incidence of AF increased from less than $0.1 \%$ per year in those under 40 years of age to greater than $1.5 \%$ per year in women over 80 years of age and greater than $2 \%$ per year in men over 80 years of age. The age-adjusted incidence increased over a 30-year period in the Framingham Study [8].

Figure 3.7 shows the clinical correlations between different risk factors that are observed in the cardiology clinical practice. Heart failure (HF) is very highly associated with the risk of developing AF, with a hazard ratio approaching 5 for men and almost 6 for women. The older the patients get, the more likely patients are at risk of developing AF. Clearly, the presence of valvular heart disease is also associated with an increased risk of developing AF. Hypertension and diabetes are really the main drivers for development down the road of AF. To some extent, myocardial infarction (MI) and coronary disease are associated with AF [70].

On the other hand, AF contributes to worsening the prognosis of patients who have other comorbidities. This is summarized in Table 3.1. In hypertensive patients, new onset AF nearly doubles the risk of cardiovascular events, triples the risk of stroke and increases the risk of hospitalization for HF by fivefold [6]. In patients with congestive heart failure (CHF), new onset $\mathrm{AF}$ increases the risk of 

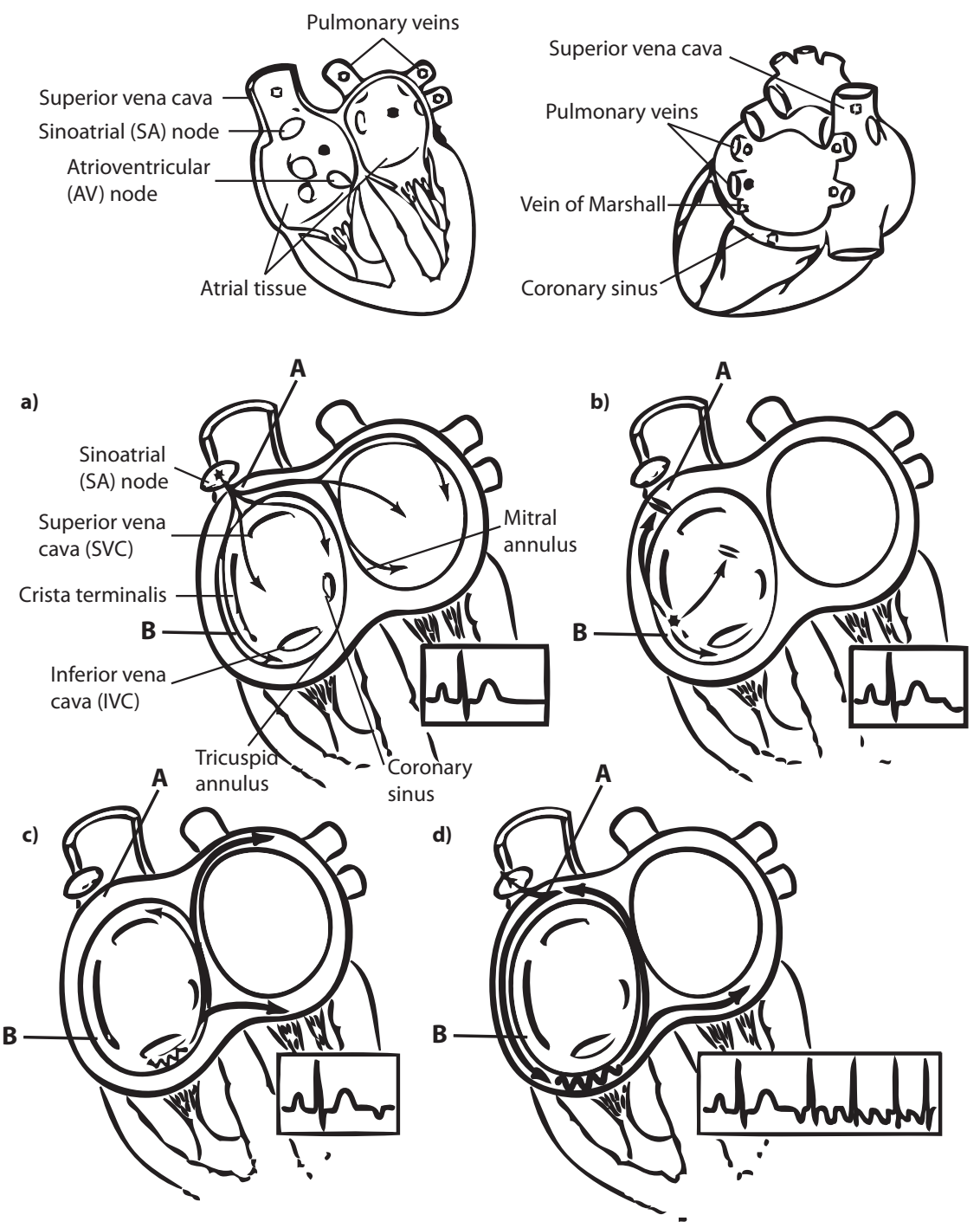

Figure 3.4. a) A sinus impulse activates area A. (b) A premature beat arising in area $B$ fails to reach area $A$ because the intervening tissue remains refractory from the preceding sinus beat. (c) The premature stimulus travels slowly via an alternative route back to area $A$, allowing enough time for area A to recover and be excited. (d) Area A re-excites area B, and the cycle sustains itself [58].

mortality by 1.6-fold in men and by 2.7 -fold in women [7]. In patients with myocardial infarction (MI), new onset AF nearly doubles the risk of in-hospital and long-term mortality [5]. Furthermore, AF increases the risk for stroke across all groups [3], and stroke associated with AF is typically more severe than stroke due to other etiologies [71, 72].

In sum, the prevalence and the incidence of the atrial fibrillation justify the 


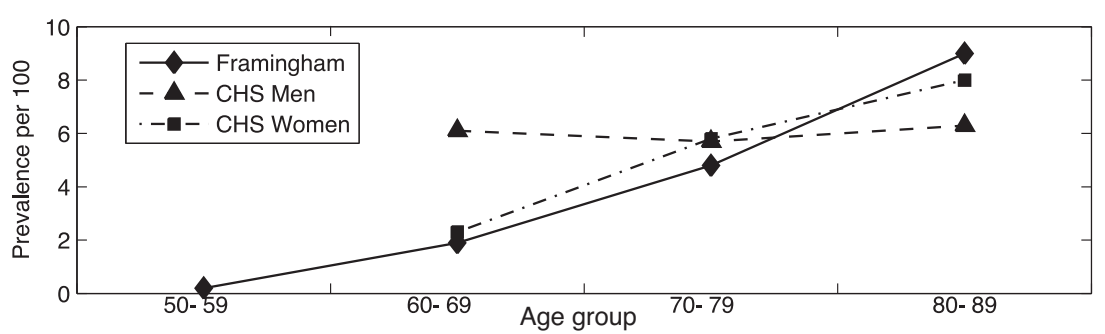

Figure 3.5. Prevalence of $\mathrm{AF}$ in two American epidemiological studies. Framingham indicates the Framingham Heart Study; CHS, Cardiovascular Health Study [1]

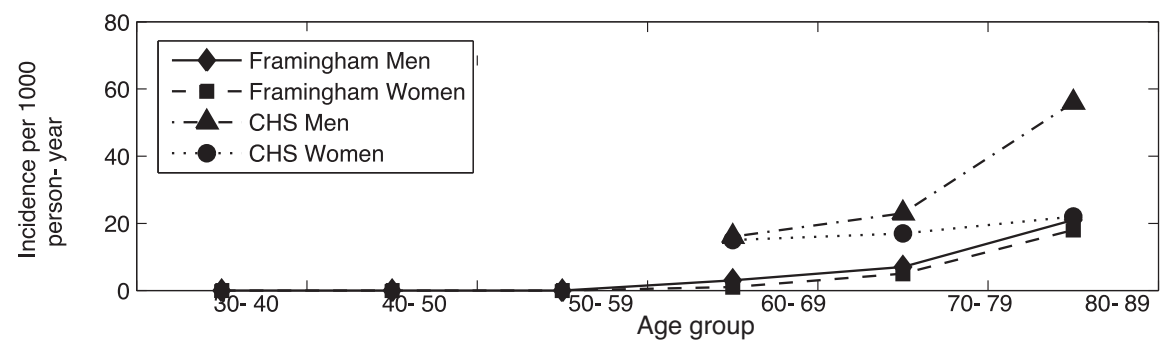

Figure 3.6. Incidence of atrial fibrillation in two American epidemiological studies. Framingham indicates the Framingham Heart Study, and CHS indicates Cardiovascular Health Study [1]

Men $(n=2090)$

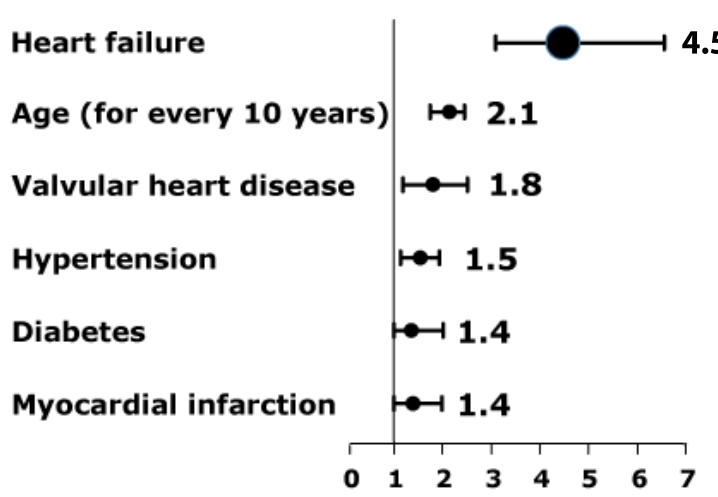

Women $(n=2641)$

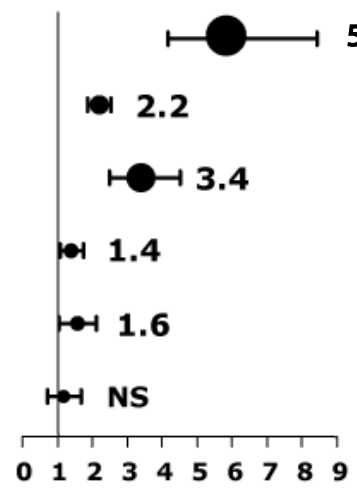

Figure 3.7. Independent risk factors for AF [70]

importance of seeking for new techniques that improve the characterization of the arrythmia in order to choose its proper diagnosis and management. It is particularly important the understanding of the mechanisms of spontaneous termination of PAF episodes because it could lead to improvements in treatment of sustained AF [21]. 


\begin{tabular}{l|l|l} 
Patients with new onset & \multicolumn{1}{|c|}{ Events } & Risk \\
AF & & \\
\hline \multirow{2}{*}{$\begin{array}{l}\text { Hypertension } \\
\text { ·n=8851 }\end{array}$} & Cardiovascular events & $\uparrow 1.88$ \\
\cline { 2 - 3 }$\cdot$ Follow-up: $4.8 \pm 1$ year & Fatal and nonfatal stroke & $\uparrow 3$ \\
\cline { 2 - 3 } CHF & Hospitalization for HF & $\uparrow 5$ \\
\hline ·n=1470, Follow-up: 5.6 years & Mortality in men & $\uparrow 1.6$ \\
\cline { 2 - 3 } $\begin{array}{l}\text { MI } \\
\cdot n=17,944, \cdot \text { Follow-up: } 4 \text { years }\end{array}$ & Mortality in women & $\uparrow 2.7$ \\
\cline { 2 - 3 } & Long-term mortality (4 years) & $\uparrow 1.78$
\end{tabular}

Table 3.1. Prognosis of patients with AF comorbidities. HF, heart failure; CHF, congestive heart Failure; Ml, myocardial infarction.

\subsection{Atrial Fibrillation and Quality of Life}

In addition to variables such as mortality and length of stay and other clinical variables, also quality of life (QoL) indicators must be considered The SF-36 score has been successfully validated and used quite frequently to describe some of these QoL concerns on the part of patients as they recover from different healthrelated experiences [4]. In figure 3.8 we show the results of this questionnaire for control patients, post-MI patients and AF patients. Unfortunately, the worst QoL parameter indicator is in patients with AF. Those patients, when they fill out the SF-36 questionnaire with regards to their general health, their social function, their mental health, and their physical function on a regular basis, reported that their QoL is actually worse than the QoL of patients who have recovered from a heart attack [4].

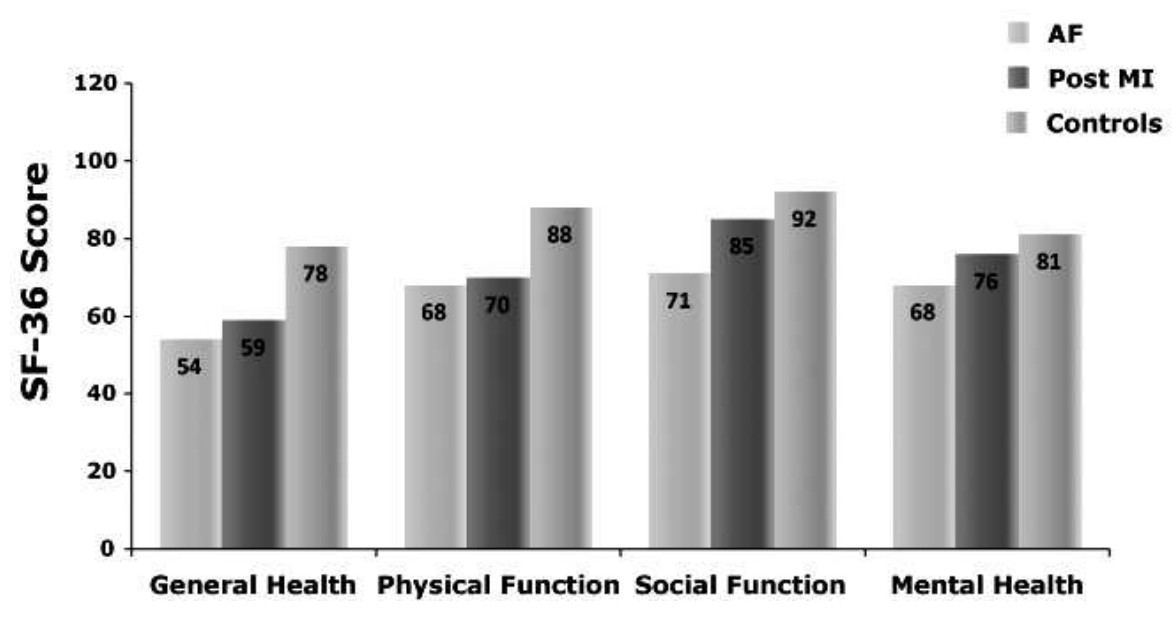

Figure 3.8. Adverse effects of AF on QoL. Lower scores mean poorer QoL [4]. 


\subsection{Bioelectric Model of Atrial Fibrillation}

\subsubsection{Potentials on the Body Surface}

Electrocardiography involves interpretation of the potentials recorded at the body surface due to electrical activity of the heart. To this end, the concept of an electrical representation of the heart's activity is used. That is, an equivalent source in conjunction with a specified volume conductor serve to model the torso [50].

There are several physical models to represent both the cardiac current sources and the enclosing torso shape and conductivity. Source models range from simple current dipoles to complex current surfaces. Torso shape and conductivity models range from infinite homogeneous conductors to finite element models. The combination of torso and source models to calculate the body surface potentials is known as the forward problem [54]. One of the most accepted solution for the forward problem relays on the computation, using surface methods, of the outer body surface potentials from the epicardial (external surface of the heart) surface potentials [73]. Surface methods are based on integral equations for the potential derived by applying Green's second identity in a torso model comprising the body surface $S_{B}$ and the heart surface $S_{H}[11,74,75]$. For solving the problem it is assumed that the region contained between the two surfaces is homogeneous. In that case, the body surface potential $\Phi_{B}$ at the $i_{t h}$ observation point can be expressed as [10, 54]:

$$
\Phi_{B}^{i}=\frac{1}{4 \pi}\left(\int_{S_{B}} \Phi_{B} d \Omega_{B B}^{i}-\int_{S_{H}} \frac{1}{r_{B H}^{i}} \nabla \Phi_{H} \cdot d \vec{S}_{H}-\int_{S_{H}} \Phi_{H} d \Omega_{B H}^{i}\right)
$$

and the heart surface potential $\Phi_{H}$ at the $i_{t h}$ observation point is:

$$
\Phi_{H}^{i}=\frac{1}{4 \pi}\left(\int_{S_{B}} \Phi_{B} d \Omega_{H B}^{i}-\int_{S_{H}} \frac{1}{r_{H H}^{i}} \nabla \Phi_{H} \cdot d \vec{S}_{H}-\int_{S_{H}} \Phi_{H} d \Omega_{H H}^{i}\right)
$$

where $r_{P Q}^{i}$ is the distance from the $i^{t h}$ arbitrary observation point on surface $P$ to one elemental source on surface $Q$, and $d \vec{S}_{H}$ is the outward differential normal vector to surface $S_{H}$. In addition, the differential solid angle $d \Omega_{P Q}^{i}$ is defined as that subtended, at the $i^{\text {th }}$ observation point on surface $P$, by an elemental area of the integration surface $Q$. Likewise, $\Phi_{P}^{i}$ is the electric potential at the $i^{\text {th }}$ observation point on surface $P$. This situations can be seen graphically for the $i^{t h}$ observation point located on surfaces $S_{B}$ and $S_{H}$ in Figures 3.9 and 3.10, respectively.

The general approach for finding solutions to this kind of integral equations is to discretize the problem and write one equation for a number of points on both 


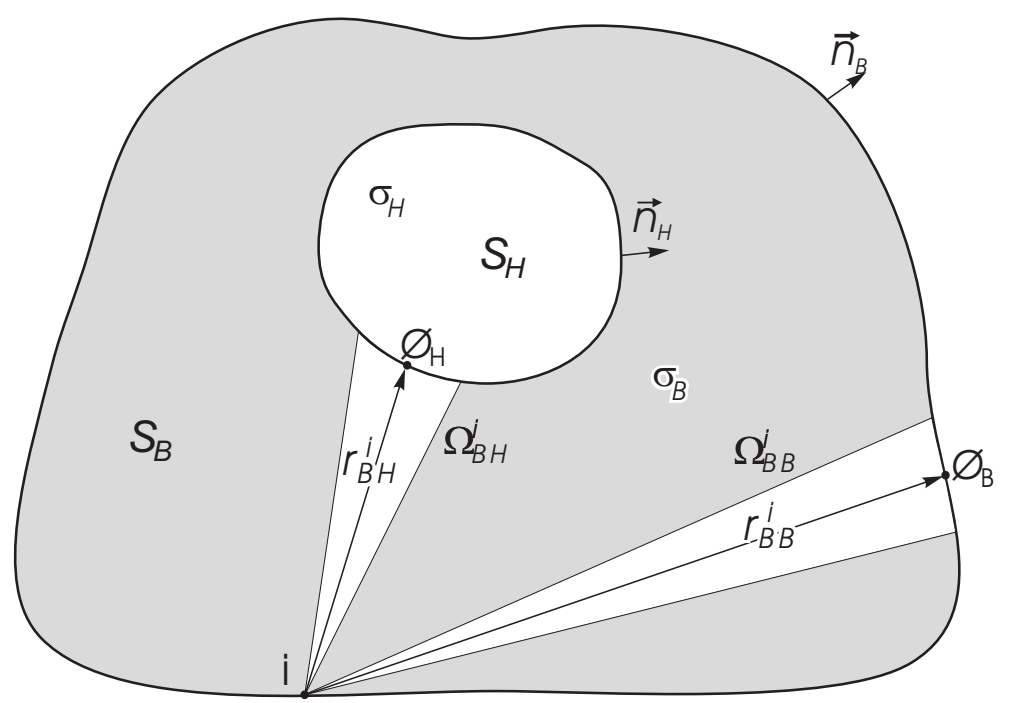

Figure 3.9. Integration surfaces and geometry outline involved in the forward problem solution when the observation point $i$ is placed on the inner bounding of the body surface $S_{B}$ [10].

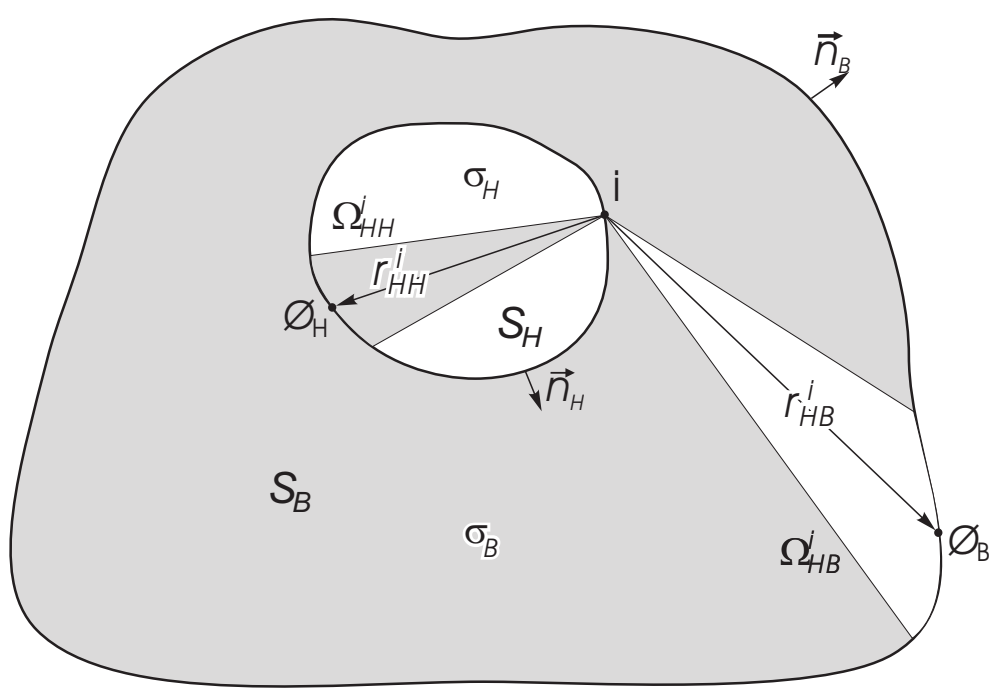

Figure 3.10. Integration surfaces and geometry outline involved in the forward problem solution when the observation point $i$ lies on the outer bounding of the heart surface $S_{H}$ [10].

surfaces and solve these equations simultaneously [54]. For $N_{B}$ points defined on the body surface, representing the field points (leads), and $N_{H}$ on the epicardium representing the source positions, it is possible to write the following set of discretized expressions as the observation point sweeps all the body and the heart surface [10, 54]: 


$$
\begin{aligned}
& \sum_{j=1}^{N_{B}} p_{B B}^{i j} \Phi_{B}^{j}+\sum_{j=1}^{N_{H}} p_{B H}^{i j} \Phi_{H}^{j}+\sum_{j=1}^{N_{H}} g_{B H}^{i j} \Gamma_{H}^{j}=0 \\
& \sum_{j=1}^{N_{B}} p_{H B}^{i j} \Phi_{B}^{j}+\sum_{j=1}^{N_{H}} p_{H H}^{i j} \Phi_{H}^{j}+\sum_{j=1}^{N_{H}} g_{H H}^{i j} \Gamma_{H}^{j}=0
\end{aligned}
$$

where $\Gamma_{H}^{j}$ is the normal component of the potential gradient for point $j$ on the heart surface. In general the $g_{P Q}^{i j}$ term links the potential at observation point $i$ on surface $P$ to the value of the potential gradient $\Gamma_{H}^{j}$ at point $j$ on surface $Q$, while $p_{P Q}^{i j}$ is the geometrical coefficient which weights the contribution in the observation point $i$ on surface $P$ of the potential at node $j$ on surface $Q$. Therefore, the equations can be separated into the product of a potential $\left(\Phi_{B}^{j}\right.$ or $\left.\Phi_{H}^{j}\right)$ or the gradient of a potential $\left(\Gamma_{H}^{j}\right)$ at a specific point $j$ on either one of the surfaces and a second factor (the terms with general form $p_{P Q}^{i j}$ and $g_{P Q}^{i j}$ ) based entirely on the geometry of the torso and the heart. $\Phi_{B}^{j}$ and $\Phi_{H}^{j}$ are the potential at node $j$ on the body and heart surfaces, respectively. Equations 3.4 and 3.4 can be rewritten in matrix form as:

$$
\begin{gathered}
\mathbf{P}_{B B} \boldsymbol{\Phi}_{B}+\mathbf{P}_{B H} \boldsymbol{\Phi}_{H}+\mathbf{G}_{B H} \boldsymbol{\Gamma}_{H}=0 \\
\mathbf{P}_{H B} \boldsymbol{\Phi}_{B}+\mathbf{P}_{H H} \boldsymbol{\Phi}_{H}+\mathbf{G}_{H H} \boldsymbol{\Gamma}_{H}=0
\end{gathered}
$$

where $\boldsymbol{\Phi}_{B}$ and $\boldsymbol{\Phi}_{H}$ are $N_{B}$ and $N_{H}$ potential column vectors, $\boldsymbol{\Gamma}_{H}$ is a column vector of $N_{H}$ epicardial potential gradients, and the various $\mathbf{P}$ and $\mathbf{G}$ coefficient matrices are determined solely by integrations involving the geometry of the epicardial and body surfaces. Here again, the first subscript of $\mathbf{P}$ (or $\mathbf{G}$ ) represents the surface containing the observation points, having as much rows as points $\left(N_{H}\right.$ or $\left.N_{B}\right)$, and the second one, the surface (heart or body) of integration with the number of columns equal to the number of points where the integration is computed $\left(N_{H}\right.$ or $\left.N_{B}\right)$. Solving Eq. (3.6) for the matrix of epicardial potential gradients $\Gamma_{H}$ and substituting the result into Eq. (3.5) yields [54, 10]:

$$
\mathbf{\Phi}_{\mathbf{B}}=\mathbf{T}_{B H} \boldsymbol{\Phi}_{H}
$$

with $\mathbf{T}_{B H}$ defined as

$$
\begin{aligned}
\mathbf{T}_{B H}= & \left(\mathbf{P}_{B B}-\mathbf{G}_{B H}\left(\mathbf{G}_{H H}\right)^{-1} \mathbf{P}_{H B}\right)^{-1} \\
& \cdot\left(\mathbf{G}_{B H}\left(\mathbf{G}_{H H}\right)^{-1} \mathbf{P}_{H H}-\mathbf{P}_{B H}\right)
\end{aligned}
$$


Eq. (3.7) and (3.8) define the solution to the forward problem. The elements of matrix $\mathbf{T}_{B H}$ are the transfer coefficients relating the potential at a particular point on the epicardial surface to that at a particular point on the body surface, and they depend solely of the geometry of the epicardial and body surfaces and the conductivity of the torso.

Eq. (3.7) shows that the electric potential in one point of the body surface can be obtained by adding the partial contributions of the heart potentials, weighted by a transfer coefficient. Obviously, Eq. (3.7) relates to a linear mixing model where a set of observations are obtained by linearly combining a set of sources. In our case, the sources are the set of bioelectric potentials in the epicardium and the observations the set of body-surface potentials.

The transfer (or mixing) matrix of Eq. (3.8), models the conductivity of the human torso and, in a first approximation, may be considered as an isotropic homogeneous volume conductor. A more realistic modeling of the torso can consider inhomogeneities of the volume conductor and the presence of different tissues. One can take such inhomogeneities into account by approximating the volume conductor by a collection of regions, each one of which is homogeneous, resistive, and isotropic but, at the same time retaining the results of Eq. (3.7) [73]. Hence, inhomogeneities and anisotropies in the human torso only modify the transfer coefficients, i.e. the elements of $\mathbf{T}_{B H}$, but do not affect the fulfillment of the model [76].

The solution of the forward problem attained in equation 3.8 is a linear expression and no other additional constraints can be inferred from it. Nonetheless, several constraints can be added to the mixing model within the condition of linearity. The most simple condition is to consider that time-varying bioelectric currents and voltages in the human body can be examined with the conventional quasi-static approximation so that the mixture of contributions to the potential in one point of the body surface occurs instantaneously. This quasi-estationarity model has been successfully used in previous works to study the ECG genesis in atrial fibrillation episodes [10]. A more complex model consists of considering that the linear mixtures described by equation 3.8 do not occur instantaneously but there exist some contribution delays in the generation of the ECG leads. In fact, time delays of several miliseconds are always perceived between the QRS maxima of different ECG leads, as exemplified in Figure 3.11. Accordingly to equation 2.4, the heart vector is a three-dimensional time-varying vector whose projections on the leads produce the electrocardiographic signals. As a consequence, the maximum projection value during the QRS complex is not obtained simultaneously in all leads. These time delays between fiducial points of ECG leads could be due to the fact that the maximum projection of the heart vector on each lead happens at different moments, which provokes a misalignment of QRS complexes.

On the other hand, let us consider the general formulation for the electric field in a infinite volume conductor due to an impressed current density source 
$\vec{J}_{s}$ whose temporal behavior is harmonic at an angular frequency $\omega$. The medium is assumed to be linear, homogeneous and isotropic and characterized by its magnetic permeability $\mu$, electric conductivity $\sigma$ and dielectric constant $\epsilon$. The electric and magnetic fields are found by solution of the inhomogeneous Helmholtz equations. The scalar potential $\Phi$ and the magnetic potential vector $\vec{A}$ are given by [17]:

$$
\begin{gathered}
\Phi\left(\vec{r}_{S}\right)=\frac{1}{4 \pi(\sigma+j \omega \epsilon)} \int_{V} \frac{1}{r} \rho\left(\vec{r}_{V}\right) \cdot e^{-j k r} d V \\
\vec{A}\left(\vec{r}_{S}\right)=\frac{\mu}{4 \pi} \int_{V} \vec{J}_{S}\left(\vec{r}_{V}\right) \cdot e^{-j k r} d V
\end{gathered}
$$

where $\vec{r}_{S}$ indicates the position where $\Phi$ and $\vec{A}$ are figured out, $\vec{r}_{V}$ indicates a point of the integration volume, $r$ is equal to $\left|\vec{r}_{V}-\vec{r}_{S}\right|$, and $k$ is the propagation constant defined as:

$$
k^{2}=\omega^{2} \mu \epsilon_{c}=\omega^{2} \mu(1+\sigma / j \omega \epsilon)
$$

and $\epsilon_{c}$ is a complex dielectric constant, implicitly defined in equation 3.11as $\epsilon_{c}=$ $\epsilon(1+\sigma / j \omega \epsilon)$, that includes the effects of conductivity and losses in its real and imaginary parts, respectively. An alternate form of writing equation 3.11 is:

$$
k^{2}=-j \omega \mu \sigma_{c}=-j \omega \mu \sigma(1+j \omega \epsilon / \sigma)
$$

where the complex conductivity is defined as $\sigma_{c}=\sigma(1+j \omega \epsilon / \sigma)$.

The time required for changes in the source to a field point is represented in equations 3.9 and 3.10 by the phase delay $e^{-j k r}$. This term can be developed as:

$$
\boldsymbol{e}^{-j k r}=1-j k r-j \frac{(k r)^{2}}{2 !}-j \frac{(k r)^{3}}{3 !} \cdots
$$

Therefore, the propagation delay of a bioelectric wave from the source point to the measure point can only be neglected when the condition $k r \ll 1$ is accomplished. In order to evaluate an upper bound of the magnitude $k r$, the typical propagation values for the human body are chosen, namely $r_{\max }=1 \mathrm{~m}, f_{\max }=1 \mathrm{kHz}, \mu=$ $4 \pi \cdot 10^{-7} \mathrm{H} / \mathrm{m}$, and $\sigma=0.02 \mathrm{~S} / \mathrm{m}$ [17]. On the other hand, setting the value of $1+j \omega \epsilon / \sigma)$ equal to the conservative value of $\sqrt{2}$ yields the maximum value of $k r$ as [17]:

$$
k r_{\text {max }}=(1-j) \sqrt{2 \pi \cdot 1000 \cdot 4 \pi 10^{-7} \cdot 0.2}=0.0397(1-j)
$$

That is, the value of $\cdot e^{-j k r}$ is near one, but there exits a certain error of 4 per cent. Plonsey assumes in [17] that this error is reasonably negligible, so that the instantaneous mixing model could be considered with a $4 \%$ error in the mixtures of 
bioelectric signals on the human body surface. As a consequence, the use of the instantaneous mixing model for the study of atrial fibrillation is validated in [10] by this low error. Nonetheless, the existence of this error along with the misalignment of QRS maxima, as a consequence of the different instants of maximum projection of the heart vector on every lead, justifies the study of a more complex mixing model than the instantaneous one. This new model is the convolutive linear mixing model, which is able to compensate the aforementioned time delays. The description of both mixing models, instantaneous and convolutive, will be later expanded in chapter 4

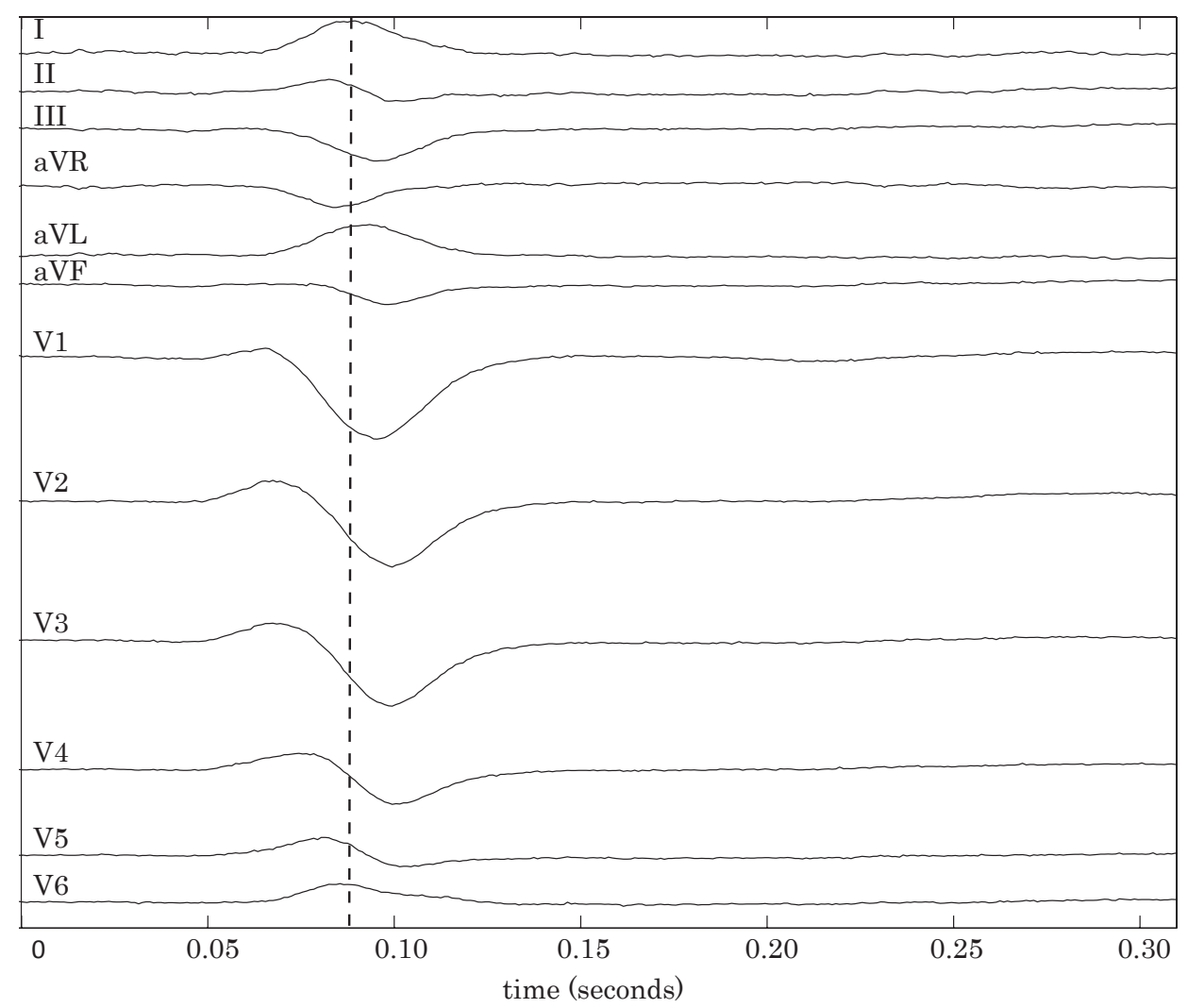

Figure 3.11. QRS complex detail of a 12-lead AF ECG. The maximum projection of the heart vector on each ECG lead occurs at different moments.

Given that the misalignment between fiducial points of ECG leads and the wave propagation delays can be taken into account in a convolutive model, this mixing model seems to be better adapted to the mixture of electrical heart sources in the human body than the instantaneous one. Furthermore, the movement of the heart during beating makes the location of sources vary and could affect the quasi-stationary assumptions considered in [10]. All these phenomena could affect in a different way to the AA extraction performance of algorithms when the instantaneous or the convolutive model are considered. In fact, one of the main 
objectives of this thesis is to study the eventual improvement of AA extraction when a model different from the instantaneous model, i.e. the convolutive model, is used to describe the mixture of heart potential sources. The study in this thesis of several convolutive algorithms will allow us to elucidate this question. The convolutive algorithms under test will be presented in chapter 4 and the results of test will be provided in chapter 7 .

\subsubsection{Independence and Nongaussianity of Atrial and Ventricu- lar Activities}

During an AF episode several independent wave fronts propagate simultaneously throughout the atria but only a reduced part of them reaches the AV node. Moreover, several properties of the AV node tend to limit strongly the ventricular activation. First, the excitability of cells within the AV node is significantly less than the atrial myocardium, thus meaning that the refractory period is considerably larger than in the atria [1]. Second, the AV node demonstrates decremental conduction properties; that is, the amplitude and rate of rise of cardiac action potentials decrease progressively from cell to cell. Because of this property, impulses may traverse only a portion of the AV node before blocking [77]. One clinical manifestation of this property is the phenomenon of concealed conduction, in which a atrial impulse that itself does not conduct to the ventricles may impair conduction of subsequent impulses, blocking the propagation of other impulses that otherwise would have conducted [77]. As a consequence of the aforementioned AV node properties most of the atrial wave fronts do not reach conduction and are not able to produce ventricular depolarization.

On the other hand, the physical origin of the atrial wave front that has been able to produce ventricular depolarization could be very variable. This uncoordinated operation of AA and VA during an AF episode makes it reasonable to regard both activities as physically independent and, in turn, as generated by statistically independent sources of cardioelectric activity. The validity of the atrio-ventricular statistical-independence assumption is in line with the findings reported by other authors in the field $[74,11,78]$.

With respect to nongaussianity, VA presents high values within the heart beat (QRS complex) and low values in the rest of the cardiac cycle. Hence, the histogram analysis of VA reveals an impulsive, i.e., supergaussian, behaviour [79] with typical kurtosis values above 15. On the other hand, AA of an AF episode has been accurately modeled as a saw-tooth signal consisting of a sinusoid with several harmonics [78], which behaves, statistically speaking, as a subgaussian random process. Moreover, when a QRS complex and T wave cancellation algorithm, like those described in [78, 79, 80, 81], is employed to cancel out VA over one ECG lead, it can be observed that the remaining ECG, mainly the AA, present a subgaussian behaviour with negative kurtosis values.

The independence and nongaussianity of source are necessary conditions for 
the applicability of the convolutive algorithms that will be later introduced in chapter 4 . This subject of applicability will be expanded in section 4.3.2. On the other hand, the consideration of QRS misalignment, the aforementioned wave propagation delay and the variable location of sources justify the performance study of convolutive algorithms. 



\section{Chapter 4 \\ Estimation Strategies of the Atrial Activity from the ECG}

4.1 Time-domain Based Techniques $\ldots \ldots \ldots \ldots \ldots 4$

4.2 Blind Source Separation . . . . . . . . . . . . . . 46

4.2.1 General Mixing Model . . . . . . . . . . . . . . . . . 46

4.2.2 Instantaneous Linear Mixing Model . . . . . . . . . . . . . . . 47

4.2.3 Convolutive Linear Mixing Model . . . . . . . . . . . . 50

4.2 .4 Global Matrix G . . . . . . . . . . . . . . . . 51

4.3 Applicability of BSS to Estimate the Atrial Activity . . . . . 53

4.3.1 Conditions of the Sources in BSS . . . . . . . . . . . . . . . . 53

4.3 .2 Separability and Identifiability . . . . . . . . . . . . . . 54

4.4 Separation Criteria for Convolutive BSS . . . . . . . . . 56

4.5 Convolutive BSS algorithms Under Test . . . . . . . . . 57

4.5 .1 Infomax . . . . . . . . . . . 58

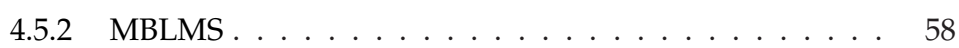

4.5 .3 TDD ........................ 60

4.5 .4 CoBliss ...................... 61

4.6 Atrial Activity Extraction from Holter Recordings . . . . . . 62

4.6.1 Inherent Problems . . . . . . . . . . . . . . . . 62

4.6.2 Wavelet Transform Principles ... . . . . . . . . 63

4.6.3 A New Algorithm for Atrial Activity Estimation: Convolutive Multiband Blind Separation . . . . . . . . 66

In the previous chapters, the basic concepts on electrocardiography and atrial fibrillation were concisely introduced. The present chapter is centered in the atrial activity (AA) extraction from ECG recordings as a necessary previous step to the exhaustive study of AF episodes. The chapter starts with a description of the 
time-domain extraction techniques used for AA extraction. Next, general concepts on Blind Source Separation (BSS) and mixing models of sources are introduced. Two main mixing models are considered. In the first place, the instantaneous mixing model is described, which has already been used for AA extraction. In the second place, the convolutive mixing model is introduced. This second model has never been used before for AA extraction, and one of the main goals of this thesis is disclosing its applicability. This goal will be accomplished by analyzing the performance of the most relevant BSS algorithms that take into account the convolutive mixing model. The algorithms which performance will be studied are presented in section 4.5. Finally, since convolutive BSS algorithms cannot be applied directly to extract the AA from Holter ECG recordings because of the lack of leads, a new algorithm for AA extraction based on the convolutive model that solves this problem is presented in section 4.6.

\subsection{Time-domain Based Techniques}

The earliest developed techniques of atrial activity estimation work in time domain, and they obtain the atrial activity by subtracting the average QRST waves from ECG signals. The most extensively used QRST Cancellation techniques in AF studies is Average Beat Subtraction (ABS) [12, 37, 79]. In ABS the successive cardiac cycles are assumed to be repetitions of the same process. Thus the registration of a heart beat can be considered as the result of an ergodic process [79]. That is, the recorded signal of a heart beat is highly correlated with the previous ones. Nevertheless, in AF episodes this regularity is only observed for ventricular components. On the contrary, since atrial depolarisation is produced by continuous and chaotic wavelet fronts, fibrillatory waves appear randomly and completely decoupled at each heart beat. As a consequence, the averaged waveform of the typical AA of AF asymptotically tends to zero. This allows to extract the average QRST template by averaging several QRST occurrences, given that when enough QRST complexes are considered, the template will be formed only by the ventricular activity [12].

ABS is performed in three main steps [79]:

1. Detection and alignment of the segments that contain the QRST wave.

2. Computation of the average beat.

3. QRST template substraction.

The problem of detection and alignment can be solved by means of thresholdbased algorithms $[82,83]$.

The ABS process is depicted in figure 4.1, which can be mathematically expressed as: 


$$
y_{a}=y-x
$$

where $y$ is one of the heart beats of the ECG, $x$ is the computed averaged beat, and $y_{a}$ is the residual signal estimated as the atrial activity.

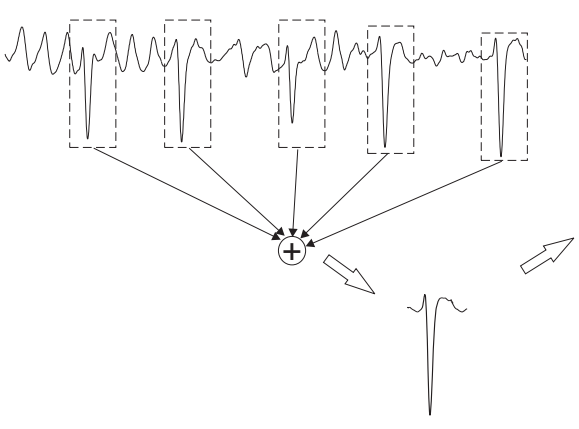

(a)

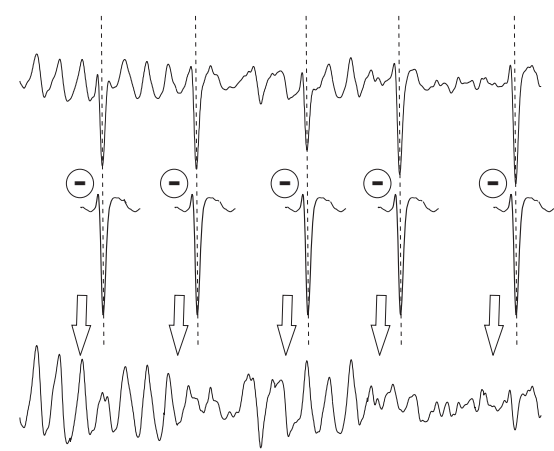

(b)

Figure 4.1. a) Computation of the QRST template of an AF episode by QRST beat averaging b) Estimation of the AA by aligning and subtracting the QRST template.

Usually, the leads that contain great atrial component are II, III, aVF and V1. V1 is frequently the lead selected to extract the atrial activity. The QRST cancellation algorithm is also referred as Template Matching and Subtraction (TMS). After the extraction of the AA by using these techniques, different measurements of autocorrelation [84] and non-uniformity [85] can be applied to the signal in order to characterize it.

In 2001, it was presented the Spatio-Temporal techniques for QRST cancellation [78], which improves the performance of the ABS method. The application of the Spatio-Temporal techniques implies to calculate a new scale factor before the subtraction of the computed averaged beat [78]. This method's performance is considerably better than the one of the straightforward ABS because it obtains a substantial reduction of the average QRST-related error. As a consequence, the quality of the extracted AA is also improved [78].

A recent modified version of ABS, the Adaptive Singular Value Cancellation (ASVC) [86, 87], studies the VA cancellation accuracy, when different complexes are selected to obtain the ventricular template. The results showed that the most correlation-similar complexes to the one under cancellation provided a more accurate ventricular cancellation template than the preceding and subsequent beats, thus providing a higher AA extraction quality. ASVC is the first cancelation strategy able to calculate the most suitable number of complexes prior to extracting the AA signal, considering the specific characteristics of the input ECG recording. In contrast, all the previously proposed ABS strategy variants considere all the ECG complexes to generate the ventricular template $[12,37,78,79,80,88,89,90]$. ASVC reaches a more accurate QRST cancellation template than those obtained 
with ABS and previous variants. In addition, ASVC is more robust in ECGs with variable QRST morphology and in the presence of ectopic beats than ABS.

\subsection{Blind Source Separation}

The aforementioned family of techniques has been well accepted and used in clinical applications [91]. Nonetheless, they are only applied to the leads where atrial fibrillation is more easily distinguishable, frequently V1. This means that, if QRST cancellation techniques is applied to $M$ different leads, $M$ different estimated atrial activities will result as well. Consequently, they do not make use of the information included in every lead in an unified way. On the contrary, Blind Source Separation (BSS) techniques [15] make a multi-lead statistical analysis by exploiting the spatial diversity introduced by multiple spatially-separated electrodes. Next, the most important models used in BSS will described.

\subsubsection{General Mixing Model}

BSS consists of retrieving a set of signals that cannot be observed directly from other set of signals that can be observed. A general scheme of the BSS problem is shown in Fig. 4.2. The signals to be retrieved are called sources, and those that can be observed are called observations. The observations are formed by the contribution of all the original sources. Two main conditions must be fulfilled in order to apply BSS techniques: 1) independence of the sources and 2) nongaussianity of the sources $[13,15,16]$. This last family of extraction techniques, when applied to the ECG, are able to obtain a set of independent sources that include a unified AA signal [11].

In the general BSS mixing model, a set o $N$ independent source signals, $\left\{s_{j} \in\right.$ $\mathbb{R}, \quad j=1,2 \ldots N\}$, are considered. After a transformation process, characterized by $M$ transformation functions $F_{i}\{\cdot\}, M$ observations, $\left\{s_{i} \in \mathbb{R}, \quad i=1,2 \ldots, M\right\}$, are recorded by $M$ sensors. That is, the general BSS model is a MIMO system (Multiple Input Multiple Output). The transformation functions $F\{\cdot\}$ are unknown, and no assumption is made on their possible linearity. Every observation can be expressed as:

$$
x_{i}=F_{i}\left(s_{1}, s_{2}, \ldots, s_{N}\right)
$$

The applicability of instantaneous BSS techniques for AA estimation in AF episodes is justified in [74]. Firstly, in AF episodes the bioelectric sources of the heart generating AA and VA are proved to be uncoupled and statistically independent, given the uncoordinated operation of AA and VA during AF episodes. Secondly, it is also proved that both activities present a non-Gaussian behavior 


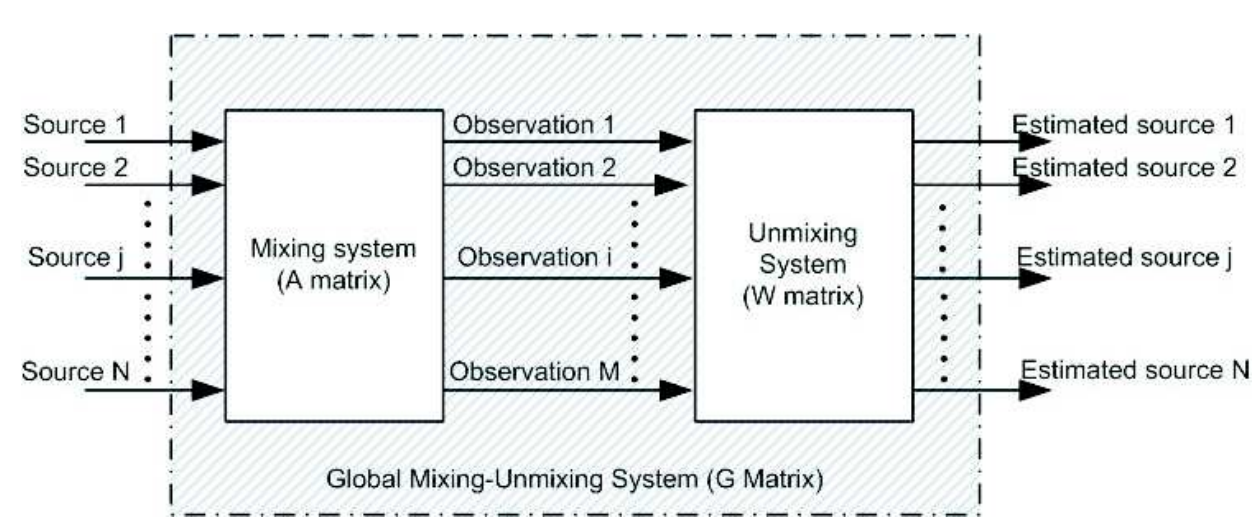

Figure 4.2. The Blind Source Separation problem. The $\bar{M}$ observed signals are function of $N$ independent sources. The sources and the transformation functions are unknown. BSS algorithms try to approximate the separation system that inverts the process. In linear models, the mixing system and the separation system are represented by the $\mathbf{A}$ and the $\mathbf{W}$ matrices respectively.

(AA has a subgaussian probability distribution whereas the VA is clearly supergaussian). Finally, it is demonstrated that ECG recordings are linear mixtures of bioelectric signals that depend on the position of the ECG electrodes. Therefore, the extraction of the AF from the ECG can be tackled as a BSS problem where the mixture of AA, VA, electric excitation due to muscular movement, undesired artifacts, noise and some other bioelectric signals produces the registered ECG signals of every lead [74], as depicted in Fig. 4.3. Two different mixing models of the bioelectric signals can be considered under the assumption of linearity. This models are described in the two following subsections.

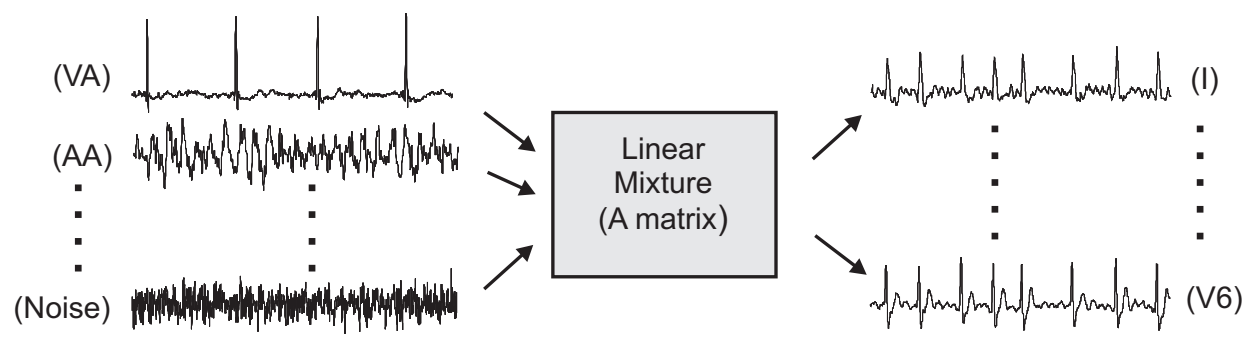

Figure 4.3. Genesis of the standard 12-leads ECG as the linear mixture of atrial activity (AA), ventricular activity (VA), noise, and other bioelectric sources.

\subsubsection{Instantaneous Linear Mixing Model}

The most frequent assumption is that the transformation functions $F\{\cdot\}$ of equation (4.2) are linear. Within the assumption of linearity, the simplest situation is the instantaneous linear model. In this case, the instantaneous mixture of the cardioelectric sources in the human body is assumed. Let us assume working with discrete-time signals and let $n$ be the sampleindex. Hence the observations $x_{i}$ can 
be expressed as [14]:

$$
\begin{aligned}
& x_{1}[n]=a_{11} s_{1}[n]+a_{12} s_{2}[n]+\cdots a_{1 N} s_{N}[n]+n_{1}[n] \\
& x_{2}[n]=a_{21} s_{1}[n]+a_{22} s_{2}[n]+\cdots a_{2 N} s_{N}[n]+n_{2}[n] \\
& \begin{array}{lll}
\vdots & \vdots & \vdots
\end{array} \\
& x_{N}[n]=a_{M 1} s_{1}[n]+\cdots \cdots+a_{M N} s_{N}[n]+n_{M}[n]
\end{aligned}
$$

where $\left\{s_{1}[n], \ldots, s_{N}[n]\right\}$ are the original sources, $\left\{x_{1}[n], \ldots, x_{M}[n]\right\}$ are the observations (i.e, the registered signals of every ECG lead), and $\left\{n_{1}[n], \ldots, n_{M}[n]\right\}$ are additive noise signals that contaminate the observations. That is, the observed signals are weighted additions of the sources plus additive noise.

This can be written in a more compact expression as [14]:

$$
x_{i}[n]=\sum_{j=1}^{N} a_{i j}[n] \cdot s_{j}[n]+n_{i}[n], \quad i=1,2, \ldots, M
$$

Using matrix notation, the previous expression can be written as [14]:

$$
\mathbf{x}[n]=\mathbf{A} \cdot \mathbf{s}[n]+\mathbf{n}[n]
$$

where $\mathbf{A}$ is a matrix that contains the $a_{i j}$ coefficients, $\mathbf{x}[n]=\left[x_{1}[n], x_{2}[n], \ldots\right.$, $\left.x_{M}[n]\right]^{T}$ is the column vector that contains the observations, and $\mathbf{s}[n]=\left[s_{1}[n], s_{2}[n]\right.$, $\left.\ldots, s_{N}[n]\right]^{T}$ contains the sources. This model can be simplified by considering the additive noise as an additional source. Hence equation 4.5 can be rewritten as:

$$
\mathbf{x}[n]=\mathbf{A} \cdot \mathbf{s}[n]
$$

The BSS algorithms based on the instantaneous linear mixing model try to estimate a $\mathbf{W}$ matrix that approximates the inverse of the mixing matrix $\mathbf{A}$ so that the independent sources can be estimated [14] as a linear transformation:

$$
\hat{\mathbf{s}}[n]=\mathbf{W} \cdot \mathbf{x}[n]
$$

where $\hat{\mathbf{s}}[n]$ is the set of estimated sources. The instantaneous linear mixing model is depicted in Fig. 4.4. The approach to the BSS problem exposed in equation 4.6, where instantaneous mixture is assumed, is known as Independent Component Analysis (ICA).

One important BSS algorithm that follows the instantaneous mixing model of ICA is the FastICA algorithm. The FastICA algorithm [14], based on the instantaneous mixing model, has already been applied to the extraction of the AA 
from ECGs of AF episodes [92]. In FastICA it is assumed that no propagation delay of the cardioelectric signals exists in the human body. The error introduced by FastICA as a consequence of assuming instantaneous mixtures resulted to be negligible and quite good results in the extraction of the AA have been obtained [74]. This algorithm has been combined with spatio-temporal techniques improving its results [93].

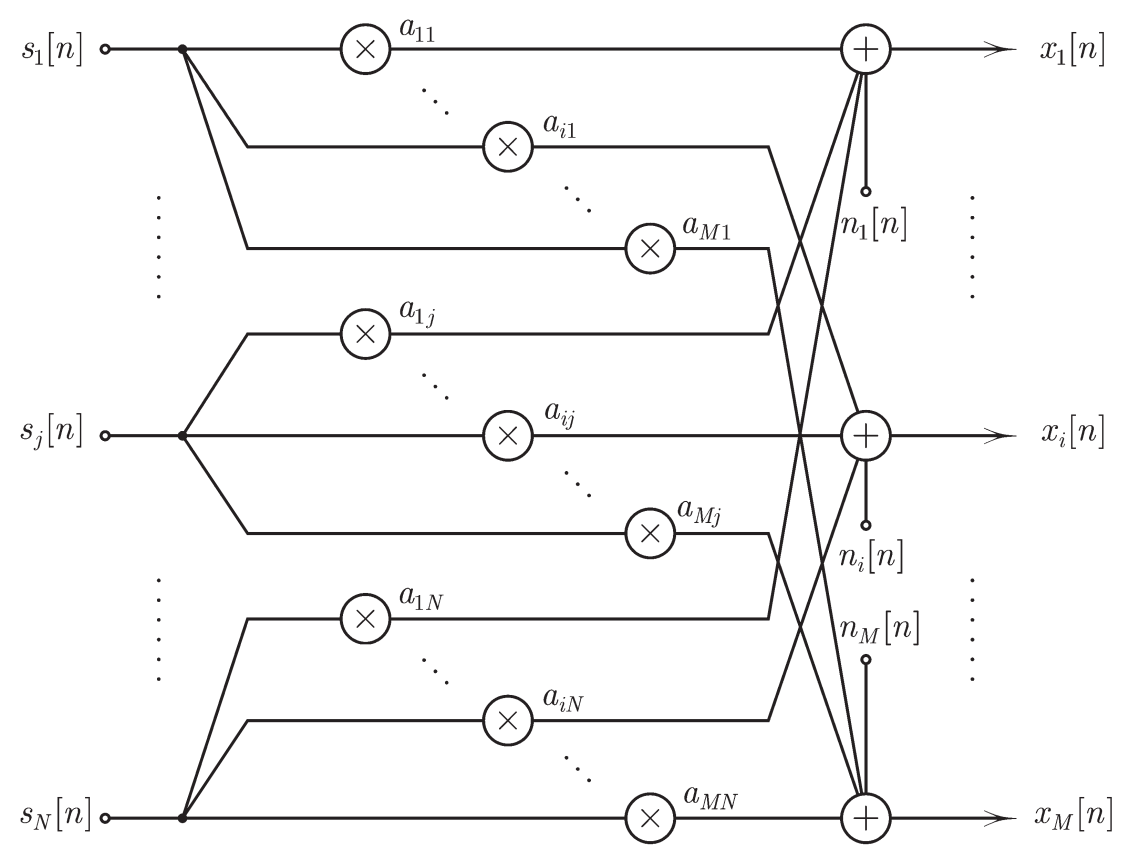

Figure 4.4. Instantaneous linear mixing model. Here the observations of the ECG are assumed to be instantaneous linear mixtures of the bioelectric sources.

The FastICA algorithm tries to find a direction as a unit vector $\mathbf{w}$ so that the projection $\mathbf{w} \mathbf{z}^{T}$ maximizes its nongaussianity. Nongaussianity is here measured by the approximation of negentropy [14]. FastICA is based on a fixed-point iteration scheme for finding a maximum of nongaussianity. FastICA uses negentropy to combine the superior algorithmic properties resulting from the fixed-point iteration with the preferable statistical properties due to negentropy [14]. The negentropy of a random variable $y$ can be approximated as[14]:

$$
J(y) \propto\left[\mathbf { E } \left[\{G(y)\}-\mathbf{E}[\{G(\nu)\}]^{2}\right.\right.
$$

where $\mathbf{E}\{\cdot\}$ is the expectation operator [94] and there is assumed that $y$ is of null mean and unit variance, $\nu$ is a gaussian variable of null mean and unit variance, and $G(\cdot)$ is a non-quadratic function. The following choices of $G(\cdot)$ have been proved to be very useful approximations: 


$$
\begin{gathered}
G_{1}(y)=\frac{1}{a_{1}} \log \cosh a_{1} y \\
G_{2}(y)=-\exp \left(\frac{-y^{2}}{2}\right)
\end{gathered}
$$

where $1 \leq a_{1} \leq 2$ is some suitable constant, often taken equal to one [14].

\subsubsection{Convolutive Linear Mixing Model}

A more realistic assumption on the mixture of sources is the so called convolutive linear mixing model. This is the model that the convolutive BSS (CBBS) algorithms presuppose. In this case, weighted and, besides, delayed contributions in the generation of the observed signals are taken into account $[95,96]$.

As shown in Fig. 4.5, the convolutive model is a more general linear mixture BSS model where previous coefficients $a_{i j}$ have been substituted by FIR (Finiteduration Impulse Response) filters $h_{i j}$ of length L. In fact, the previous instantaneous model could be considered as a particular case of the convoulutive model where L equals one. Hence, the convolutive linear mixing BSS model can be expressed as:

$$
x_{i}[n]=\sum_{j=1}^{N} h_{i j}[n] * s_{j}[n]+n_{i}[n], \quad i=1,2, \ldots, M
$$

where $*$ is the convolution operator. Also here, it is considered the presence of additive noise in the observations. Using matrix notation, equation 4.11 can be rewritten as:

$$
\mathbf{x}[n]=\mathbf{A} * \mathbf{s}[n]+\mathbf{n}[n]
$$

If the additive noise is considered as an additional source, equation 4.12 is rewritten as a simpler expression:

$$
\mathbf{x}[n]=\mathbf{A} * \mathbf{s}[n]
$$

Similarly to the instantaneous case, the linear transformation that estimates the original independent sources in the convolutive case is given by:

$$
\hat{\mathbf{s}}[n]=\mathbf{W} * \mathbf{x}[n]
$$

where $\mathbf{W}$ is an estimation of $\mathbf{A}^{-1}$. 


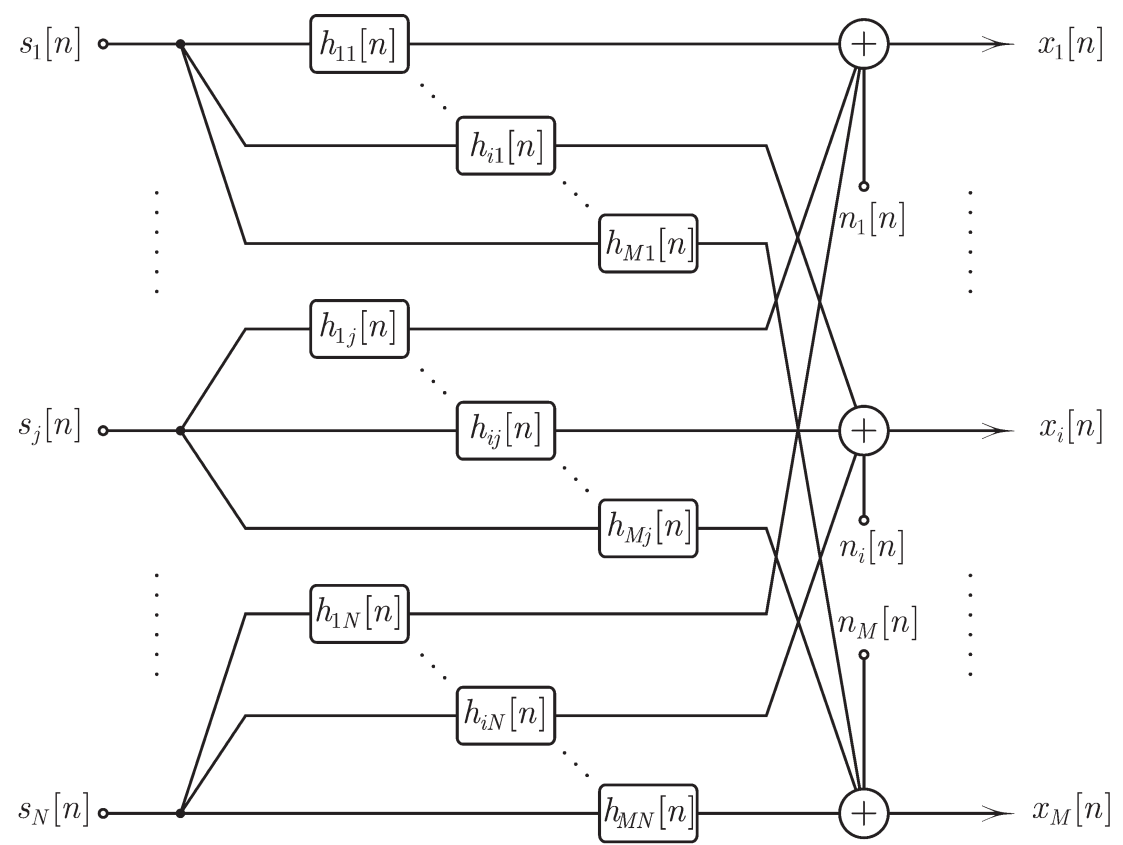

Figure 4.5. Convolutive linear mixing model. Here the observations of the ECG are assumed to be convolutive linear mixtures of the bioelectric sources.

\subsubsection{Global Matrix G}

Given that both the mixture and separation of signals are characterized by their respective matrices $\mathbf{A}$ and $\mathbf{W}$, the general process where the mixture and the separation processes are executed sequentially can be expressed as a global mixingunmixing matrix $\mathbf{G}$ which is the product of $\mathbf{A}$ and $\mathbf{W}$. In the case of the instantaneous mixture model, the matrix $\mathbf{G}$ is expressed as:

$$
\mathbf{G}=\mathbf{W} \cdot \mathbf{A}
$$

and the estimated sources are given by:

$$
\hat{\mathbf{s}}[n]=\mathbf{W} \cdot \mathbf{A} \cdot \mathbf{s}[n]=\mathbf{G} \cdot \mathbf{s}[n]
$$

The ideal solution of the BSS problem for the instantaneous model is to find the unmixing matrix $\mathbf{W}$ that equals the inverse of $\mathbf{A}$ so that $\mathbf{G}$ matches exactly the identity matrix I. In this case, the estimated sources would exactly match the original sources, since it is accomplished that:

$$
\hat{\mathbf{s}}[\mathbf{n}]=\mathbf{G} \cdot \mathbf{s}=\mathbf{I} \cdot \mathbf{s}=\mathbf{s}[n]
$$


where $\mathbf{s}[n]$ is the set of original sources, $\hat{\mathbf{s}}[n]$ is the set of estimated sources, and $\mathbf{x}[n]$ is the set of observations. Similar expressions describe the global mixingunmixing process when the convolutive mixture model is considered. In this case the global matrix is written as:

$$
\mathbf{G}=\mathbf{W} * \mathbf{A}
$$

and the estimated sources as:

$$
\hat{\mathbf{s}}[n]=\mathbf{W} * \mathbf{A} * \mathbf{s}[n]=\mathbf{G} * \mathbf{s}[n]
$$

In the convolutive case, the matrices $\mathbf{A}, \mathbf{W}$ and $\mathbf{G}$ are not constituted by unique coefficients but by FIR filters instead. The global matrix, $\mathbf{G}$, must ideally tend to a matrix where all FIR filters of the same row except one have null response. The ideal response of the non-null filter is a Dirac delta. Figure 4.6 shows an example of ideal $2 \times 2$ global matrix , G, with FIR filters length equal to 15 .
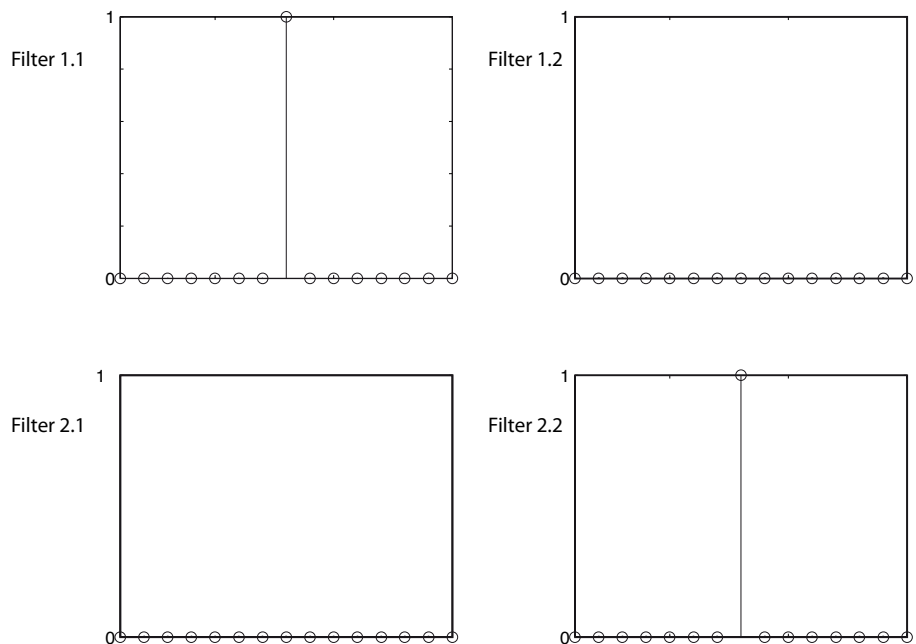

Figure 4.6. Ideal global matrix $\mathbf{G}$ with FIR filters length equal to 15 . The elements of the main diagonal, i.e. filters 1.1 and 2.2, are Dirac deltas. The rest of elements are filters of null response.

The mixing process is only reversible under certain conditions. Let us first consider the simplest case, when two sources and two observations are considered. In the instantaneous model, the ideal matrix $\mathbf{W}$, i.e. the inverse of $\mathbf{A}$, is given by:

$$
\mathbf{W}=\mathbf{A}^{-\mathbf{1}}=\frac{1}{h_{11} h_{22}-h_{21} h_{22}}\left[\begin{array}{lr}
h 22 & -h 21 \\
-h 12 & h 11
\end{array}\right]
$$

Thus $\mathbf{A}$ is not singular, i.e. its inverse exists, if and only if it is accomplished the condition: 


$$
h_{11} h_{22}-h_{21} h_{22} \neq 0
$$

In other words, the determinant of A must not be zero. Similarly, in the convolutive model, the inverse of the mixing matrix $\mathbf{A}$ is computed as [97]:

$$
\mathbf{W}=\mathbf{A}^{-1}=\frac{1}{h_{11} * h_{22}-h_{21} * h_{22}}\left[\begin{array}{lr}
h 22 & -h 21 \\
-h 12 & h 11
\end{array}\right]
$$

Here, $h_{i j}$ are FIR filters and $\mathbf{A}$ is not singular if and only if:

$$
h_{11} * h_{22}-h_{21} * h_{22} \neq 0
$$

If there are more observations than sources, as often happens in AA extraction, then $\mathbf{A}$ is not a square matrix. Hence $\mathbf{A}$ has not maximum rank so that its inverse does not exist. Consequently, $\mathbf{W}$ is figured out as a LMS (Least Minimum Square) approximation of the so called pseudoinverse matrix[14]:

$$
\mathbf{W}=\left(\mathbf{A}^{H} \mathbf{A}\right)^{-1} * \mathbf{A}^{T}
$$

where $\mathbf{A}^{H}$ is the transposed conjugated matrix of $\mathbf{A}$. The less restrictive condition that now must be fulfilled is that the inverse of $\left(\mathbf{A}^{H} \mathbf{A}\right)$ exists.

\subsection{Applicability of BSS to Estimate the Atrial Activ- ity}

\subsubsection{Conditions of the Sources in BSS}

The application of convolutive BSS is conditioned to the accomplishment of a set of underlying assumptions on the source signals. The principal assumptions on sources $\left\{s_{j}[n]\right\}, \quad j=1, \ldots, N$ are stationarity, null mean, and mutual independence [98]. Although most of signals do not comply with the condition of null mean, this condition of null mean can be always reached by signal preprocessing. The condition of independency implies that, for every pair of samples $s_{1}=s_{i}[n]$ and $s_{2}=s_{j}[l]$ of any pair of mixed source signals, the joint density function of $s_{1}$ and $s_{2}$ can be factorized as the product of the marginal density functions:

$$
p_{s_{1} s_{2}}=p_{s_{1}} p_{s_{2}}
$$

The statistic independence of every source signal with the other sources is a paramount requirement for most formulations of convolutive BSS problem. Nev- 
ertheless, the accomplishment of this condition does not guarantee the separation. Every separation method establishes additional requirements on the source signals.

\subsubsection{Separability and Identifiability}

When the BSS problem is tackled, the first subject to deal with is to know the conditions that a mixing matrix must obey to ensure the feasibility of the sources separation. This is related with the concept of separability [13] and exclusively depends on the mixing matrix $\mathbf{A}$. In the particular case where the number of observations is equal to the number of sources, separability is guaranteed if the mixing matrix is not singular. In this case, the lineal transformation associated to the mixture is a biyective relation so that $\mathbf{A}$ is regular and its inverse can always be computed as $\mathbf{W}=\mathbf{A}^{-1}$. On the contrary, if $\mathbf{A}$ is singular, the correspondence is not biyective and, therefore, the inverse of the mixing matrix can not be computed. A necessary and sufficient condition for the separability of the mixture is that its inverse system exist, and this is guaranteed if $\mathbf{A}(z)$ is of complete rank by columns in the unit circle $|z|=1$.[99]

Another important question is the identifiability of the mixture, also called principle of separation. Both terms refer to the possibility of determining the mixing matrix. It would never be possible to exactly determine the mixing matrix; instead, a certain ambiguity will always exist.

Two hypotheses have been fixed as principles of separation, accordingly to theorem of Darmois-Skitovich: [95]:

- Principle 1: If no more than one source is gaussian, the two-by-two independence property of the observations is sufficient to guarantee the separation.

- Principle 2: In the presence of more than one gaussian source, the twoby-two independence property only guarantees the separation of the nongaussian sources.

It is proved that the separation matrix $\mathbf{W}$, that obtains two-by-two independent outputs, is not unique [16]. The solution of the BSS problem has two inherent indeterminacies,namely:[95]

- Indeterminacy 1: The property of independence is preserved for any scaling of the outputs.

- Indeterminacy 2: The rearrangement of the outputs does not modify the property of independence. 
In the convolutive case [95], it is important to note that the mixing and separation processes are linear memory systems. This fact introduces a new indeterminacy in the solution of the BSS problem, that is, the impossibility of calculating the delay related to every extracted source.

In the case of AF episodes the sources that constitute the ECG can be considered independent, given that the atrial activity and the ventricular are generated by independent bioelectric phenomena [10]. Furthermore, neither the atrial activity nor the ventricular activity are gaussian sources, but they are subgaussian and supergaussian sources, respectively [10]. On the one hand, the condition of supergaussian probability density function (pdf) of the ventricular activity is easily demonstrable by the fact that ventricular components have high amplitudes within short segments that contain QRS complexes, whereas they have small amplitudes the rest of time. Consequently, the VA has a pointed probability density function, where most values are concentrated around small values and with long tails. The typical kurtosis of VA is around 30, which confirms the supergaussianity of this random variable $[10,14,93]$. On the other hand, the AA of AF episodes approaches a saw-tooth signal [78] and behaves as a typical subgaussian random variable with typical a kurtosis around -0.5. Figure 4.7 shows the histogram and the kurtosis of the VA and the AA of an AF episode. Therefore, none of the source concerning AF episodes, i.e. AA and VA, have a gaussian pdf. Accordingly to the aforementioned principle 2, the extraction of the atrial activity of an atrial fibrillation episode can be handled as a BSS problem.

Another important factor to take into account is the presence of noise in the ECG recordings. In general, the subgaussian behavior of the estimated atrial activity is not so far from the gaussianity. Nevertheless, this fact is not considered a problem for the separation of the AA from the gaussian noise, because the power of noise in ECG can be normally reduced by signal preprocessing to much smaller values than the AA power[74].
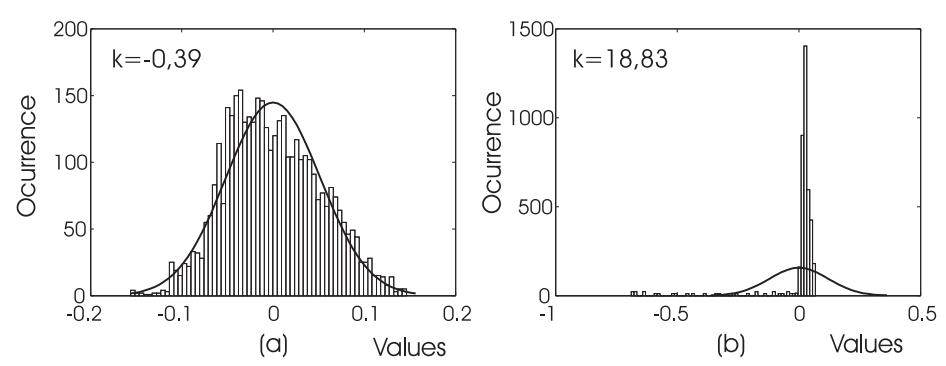

Figure 4.7. Example of histogram and kurtosis of a)AA source and b)VA source. The AA behaves as a subgaussian random variable with negative kurtosis. The VA behaves as a supergaussian random variable with possitive kurtosis. 


\subsection{Separation Criteria for Convolutive BSS}

The performance of any BSS technique is highly related with the criterion used for separation. This criterion depends on the initial assumptions on the source signals. Most of BSS methods consider that all source signals are statistically independent. Nevertheless, all methods use additional characteristic features of the source signals to reach their separation. The existing criteria for convolutive BSS can be divided into three groups: criteria based on density modeling, criteria based on contrast functions, and criteria based on correlation.

The density modelling based convolutive criteria are defined from the concepts of Information Theory (IT). The IT is applicable to a variety of fields as Communications, Economy, Neuroscience and Physics [100]. The Information Theory is useful for BSS because it can characterize the amount of information shared by a set of signals. Intuitively, the separation of signals is reached when no common information exists between any subset of source signals. The base of these methods is density modeling since they model the joint pdf of the output signal set, $\mathbf{y}[n]$, so that the separation system is adjusted to produce the most approximately independent output signal series [14]. In the last years, several mathematical formulations have been developed on the IT [101, 102]. Nevertheless, all these formulations can be unified by using the Kullback-Leibler divergence [13]. Considering the notation $\mathbf{y}[n]=\hat{\mathbf{s}}[n]$, the Kullback-Leibler divergence is expressed as:

$$
D\left(p_{y} \| \hat{p}_{y}\right)=\int p_{y}(y) \log \left(\frac{p_{y}(y)}{\hat{p}_{y}(y)}\right) d y
$$

where $p_{y}(y)$ and $\hat{p}_{y}(y)$ are the original and the estimated pdf of $\mathbf{y}[n]$. Equation 4.26 quantifies the distance between $p_{y}(y)$ and $\hat{p}_{y}(y)$. Equation 4.26 can be rewritten by using the expectation operator $\mathbf{E}\{\cdot\}$ so that:

$$
D\left(p_{y} \| \hat{p}_{y}\right)=\mathbf{E}\left\{\log \left(\frac{p_{y}(y)}{\hat{p}_{y}(y)}\right)\right\}
$$

The contrast functions are an alternative to the criteria based on density modeling [16]. The concept of a contrast function is similar to a LED of an electric or mechanic device that indicates the accomplishment of certain operation condition. Similarly, a contrast function indicate whether one output signal of a separation system, say $y_{i}[n]$, is contributed by a unique source signal, say $x_{j}[n]$. The key of this is determining the function that depends only on $y_{i}[n]$ but not on the mixture conditions. From equation 4.17 the i-th estimated source can be expressed as a function of the $g_{i j}$ elements of matrix $\mathbf{G}$ : 


$$
y_{i}[n]=\sum_{j=1}^{N} g_{i j} s_{j}[n] \quad i=1, \ldots, N
$$

A contrast function is a cost function $\Psi\left(y_{i}[n]\right)$ which is maximized when it is accomplished that [103]

$$
g_{i j}=\left\{\begin{array}{cc}
d_{l} & \text { for a unique value of } l \quad 1 \leqslant l \leqslant N \\
0 & \text { rest of cases }
\end{array}\right.
$$

This contrast function must be easy to evaluate and must identify the separation condition for the source signal statistics as a its own maximization. The normalized kurtosis is an example of contrast function able to identify a correct separation solution in the BSS problem. The normalized kurtosis $k_{y}$ of an aleatory variable $y$ is defined as [103]:

$$
k_{y}=\frac{E\left\{|y|^{4}\right\}}{E^{2}\left\{|y|^{2}\right\}}-3
$$

With reference to the correlation criteria, the decorrelation of observations was the first approach to solve the problem of convolutive mixture separation [104, 105]. The decorrelation is reached when the cross-correlation matrix of sources becomes null for any delay. Attending to this criterion, the following crosscorrelations must be cancelled when casual, L-length separation filters are considered:

$$
\mathbf{R}_{y_{i}, y_{j}}[k]=\mathbf{E}\left\{y_{i}[n] y_{j}[n-k]\right\}=0, \forall i,\left.j\right|_{i \neq j}, k=0, \ldots, L-1
$$

\subsection{Convolutive BSS algorithms Under Test}

In this section several convolutive BSS algorithms will be introduced, the AA extraction performance of which will be tested in this thesis. In order to study all range of separation criteria, four CBSS algorithms have been selected. These algorithms optimize the separation of audio sources in reverberant spaces where convolutive mixture is assumed [106] but have never been used for the extraction of bioelectric signals. First, the Infomax algorithm is described as an algorithm based on density modeling. Next the MBLMS algorithm is introduced, which is based on contrast functions. A first approach to the correlation criterion in BSS is the TDD algorithm. Finally, the CoBliSS algorithm will be described, which is also based on the correlation criterion and, in contrast to TDD, adjusts the weights alternatively in time and frequency domain. The code of all four tested algorithms was made free available for the scientific community by their respective authors. 


\subsubsection{Infomax}

In 1995, A. Bell and T. Sejnowski exposed the theory basis for using the IT in BSS [102]. The BSS algorithms based on the IT try to maximize the output entropy, or information flow, of a neural network with nonlinear outputs. This family of algorithms are referred as infomax algorithms [14].

Let us consider $\mathbf{x}$ as the input of a neural network. The outputs $y_{i}$ of the neural network are of the form:

$$
y_{i}=\phi_{i}\left(\mathbf{b}_{\mathbf{i}}^{\mathbf{T}} \mathbf{x}\right)
$$

where the $\phi_{i}$ are some nonlinear scalar functions, and the $\mathbf{b}_{\mathbf{i}}$ are the weight vectors of the neurons. $\phi_{i}$ are sigmoidal functions [107] that are used to provide the high order statistic (HOS) needed to establish the independence of sources. The objective of infomax is to maximize the entropy of the outputs [14]:

$$
H(\mathbf{y})=H\left(\phi_{1}\left(\mathbf{b}_{1}^{\mathbf{T}} \mathbf{x}, \ldots, \mathbf{b}_{\mathbf{n}}^{\mathbf{T}} \mathbf{x}\right)\right)
$$

where $H(\cdot)$ is the entropy operator. Two of the most frequently used sigmoidal functions are the logistic function

$$
\phi(y)=\frac{1}{1+\exp (-y)}
$$

and the hyperbolic function [14]

$$
\phi(y)=y \pm \tanh (y)
$$

K.Torkola extended the infomax algorithm of Bell and Sejnowski for use with delayed and convolved sources [108]. T. Amari proposed a more effective criterion of natural gradient [101], and Te-Won Lee et al extended the work of Torkola by using equations of a feedforward network architecture [109]. Asano [110] proposed a BSS algorithm for convolutive mixture that works in the time-frequency domain and wich is a combination of Principal Component Analysis (PCA) and infomax. This is a BSS algorithm that has been optimized for audio mixtures, and where PCA is employed for reducing room reverberation. This algorithm will be henceforth referred as the Infomax algorithm.

\subsubsection{MBLMS}

The LMS (Least Mean Square) algorithm is the simplest adaptive filtering algorithms that are currently used. The LMS algorithm is executed in three main 
steps [111]:

1. Filtering the input signal $x[n]$ by means of an adaptive filter.

2. Generation of the error signal $e[n]$, computed as a comparison of the filtered signal, $y[n]$, and the desired response, $d[n]$. In the case of blind deconvolution, the expected signal $d[n]$ is the original source, $s[n]$, and the filtered signal $y[n]$ is the estimated source $\hat{s}[n]$.

3. Adjustment of the filter coefficients according to the error signal.

In other words, the objective of the LMS algorithm is to minimize the meansquare error of the signal $e[n]$ that is obtained in step two. The process is illustrated in Figure 4.8 for multiple inputs and outputs. This minimization can be referenced as a cost function, $\Psi$, that evaluates the mean-square error [97]. That is:

$$
\Psi=\mathbf{E}\left\{|y[n]-s[n]|^{2}\right\}
$$

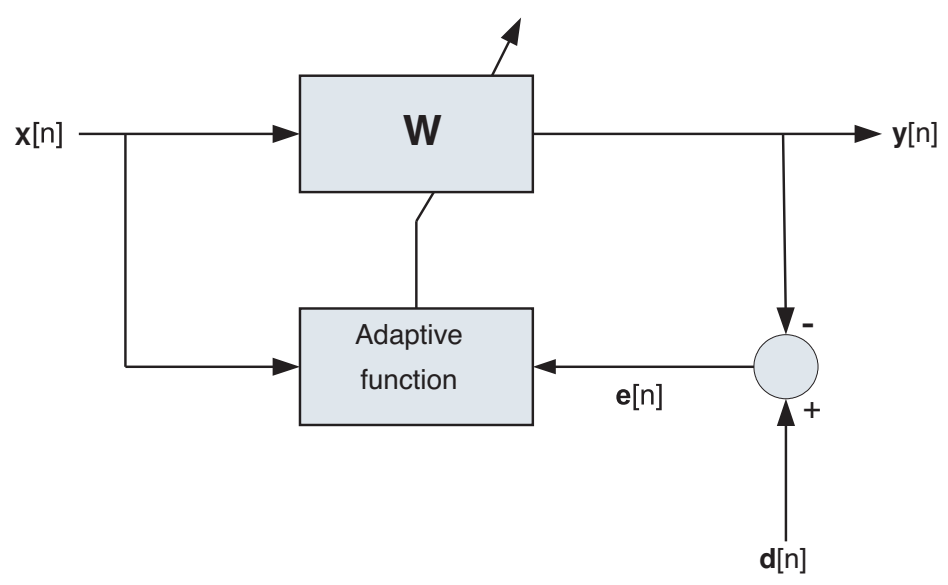

Figure 4.8. Standard LMS Algorithm

Nonetheless, the desired signal $\mathbf{d}[n]$ is unknown, given that it corresponds to a source signal of the BSS problem. Thus, the cost function $\Psi$ must be estimated. This can be done by means of the non-linear Bussgang function in order to provide the following cost function [111, 112]:

$$
\Psi=\mathbf{E}\left\{|y-g(y)|^{2}\right\}
$$

The nonlinear Bussgang function is defined as [111, 112]: 


$$
g(y)=\frac{-E\left\{y^{2}\right\} p^{\prime}(y)}{p(y)}
$$

where $p(y)$ is the density of $y$ and $p^{\prime}(y)$ its derivative.

The cost function $\Psi$ is minimized in order to adapt the deconvolution filter weights iteratively. Lambert has obtained a cost function that can be extended to the multiple inputs case $[97,113]$ :

$$
\Psi=\text { trace } \quad \mathbf{E}\left\{\left(\mathbf{y}-g(\mathbf{s})(\mathbf{y}-g(\mathbf{s}))^{H}\right\}\right.
$$

where the trace of an $N \times N$ square matrix is defined as the sum of the elements on the main diagonal. The algorithm that use the previous cost function is called the Multi-channel Blind Least-Mean Square (MBLMS) algorithm [97, 113].

\subsubsection{TDD}

The Time-Delayed Decorrelation (TDD) algorithm was first proposed by Molgedey and Schuster [104] for instantaneous mixtures, i.e. $\mathbf{x}[n]=\mathbf{A} \cdot \mathbf{x}[n]$, where $\mathbf{A}$ is a $N \times N$ matrix. The goal is to find a matrix $\mathbf{W}$ which is equivalent to the inverse matrix of $\mathbf{A}$ with the ambiguity of amplitude and permutation. The correlation matrix $\mathbf{R}_{x x}[n]$ of observations is written as:

$$
\mathbf{R}_{x x}[n]=\mathbf{A} \cdot \mathbf{E}\{\mathbf{s}[k] \mathbf{s}[k-n]\} \cdot \mathbf{A}^{T}=\mathbf{A} \cdot \mathbf{R}_{s s}[n] \cdot \mathbf{A}^{T}
$$

where $\mathbf{R}_{s s}[\tau]$ is the correlation matrix of sources.

Let us assume that the components $s[n]$ are independent and that $\mathbf{R}_{s s}[n]$ is a diagonal matrix for any value of $n$. Molgedey and Schuster demonstrated that, under these conditions, the BSS problem of finding $\mathbf{B}$ is reduced to solve the eigenvalue problem $[114,115]$ :

$$
\left(\mathbf{R}_{x x}\left[n_{1}\right] \mathbf{R}_{x x}\left[n_{2}\right]^{-1}\right) \mathbf{W}=\mathbf{W}\left(\boldsymbol{\Lambda}_{1} \boldsymbol{\Lambda}_{2}\right)
$$

This problem can also be solved by simultaneous diagonalization of time-delayed matrices. In this case, the instantaneous BSS problem is solved in two steps, namely sphering and rotation [114]. Ikeda and Murata [114, 115] proposed a BSS algorithm that extends the application of the TDD algorithm to the case of convolutive mixtures of signals. This algorithm uses the the spectrogram to transform mixed source signals into the time-frequency domain.

One main difficulty of BSS algorithms that work in time-frequency domain is the ambiguities of amplitude and permutation. In [114, 115] this is solved 
by using the inverse of the decorrelating matrices and the envelope of the signals. On the one hand, the problem of amplitude ambiguity is solved by putting back the separated independent components to the sensor input with the separation matrix $\mathbf{W}$. On the other hand, the problem of permutation is solved by the similarity among envelopes. As a result, the algorithm proposed by Ikeda and Murata $[114,115]$ obtains separated spectrograms, $S_{i}[f, n]$, which inverse Fourier transforms yield the estimated original time-domain signal sources $s_{i}[n]$. This last algorithm will be referred as the TDD algorithm that will be tested in our work.

\subsubsection{CoBlisS}

Schobben and Sommen worked in the BSS problem applied to the separation of multiple speakers in a room using multiple microphones [116, 117]. They presented a new BSS algorithm that was entirely based on Second Order Statistics (SOS), which was entitled Convolutive Blind Signal Separation (CoBliSS) algorithm. In this algorithm, the optimization is done by minimizing the crosscorrelation among the outputs of the multichannel separating filter. This criterion is transformed to the frequency domain in order to achieve a computationally inexpensive algorithm with fast convergence. The filter coefficients are figured out in the frequency domain so that the cross-correlation become equal to zero. While in TDD the approximation of coefficients is entirely made in the frequency domain, in CoBliSS, the approximation weights are adjusted iteratively in alternate time and frequency domains $[116,117]$.

Attending to equation 4.14 , the $i^{\text {th }}$ estimated source $s_{i}[n]$ of the separation process for convolutive mixtures can be expressed from the set of observations $\mathbf{x}$ as:

$$
\hat{s}_{i}[n]=\sum_{j=1}^{M} w_{i j}[n] * x_{j}[n] \quad i=1,2, \ldots, M
$$

with $\mathrm{M}$ the number of of observations and $w_{i j}$ the filters of the separation matrix W.

The cross-correlation among the outputs can be written as [116, 117]:

$$
R_{y_{i}, y_{j}}[k]=\mathbf{E}\left\{y_{i}[n] y_{j}[n+k]\right\}=\sum_{a=1}^{M} \sum_{c=1}^{M} \sum_{b=0}^{L-1} \sum_{d=0}^{L-1} w_{i a}[b] w_{j c}[d] r_{x_{a} x_{c}}[l+b-d]
$$

where 


$$
r_{x_{a} x_{c}}=\mathbf{E}\left\{x_{a}[n] x_{c}[n+d]\right\}
$$

A cost function can be formed directly from equation 4.43 using, for example, the sum of squares of the cross-correlation coefficients. The straightforward minimization of such a cost function is not eligible however due to the large number of filter coefficients involved. A typical example is that two sources and two observations are used. In that case, four FIR filters need to be calculated, each with several hundreds to thousands coefficients. Furthermore, all these coefficients are dependent on each other which makes the problem even more difficult. Therefore, an approach is required that solves for filter coefficients subsets which are as independent of each other as possible. In order to achieve this, equation 4.43 is transformed to the frequency domain, where the CoBliSS algorithm works as an iterative method [116, 117].

\subsection{Atrial Activity Extraction from Holter Recordings}

\subsubsection{Inherent Problems}

Ambulatory electrocardiography, namely Holter electrocardiography, is a widely used noninvasive test to evaluate ECG abnormalities in patients with various cardiac disease states. The clinical utility of the ambulatory ECG recording lies in the ability to continuously examine the patient's rhythm over an extended time, permitting ambulatory activity and facilitating examination of diurnal physical and physiologic changes. In contrast to the standard ECG, which provides a fixed picture of 12 leads that demonstrate cardiac electrical events over a brief period (typically less than 30 seconds), the 24-hours ambulatory ECG provides a more narrow view of 2 or 3 leads of ECG data but has the strength of recording changing dynamic cardiac electrical phenomena that often are transient and of brief duration. Ambulatory monitoring provides a record of past events, allowing detailed analysis of dynamic and transient ECG changes. Clinical experience has shown ambulatory ECG to be one of the most cost-effective clinical tools in the diagnosis and assessment of symptomatic and asymptomatic cardiac arrhythmias, prognostic assessment of risk stratification of various cardiac populations, and the evaluation of arrhythmia management modalities [118].

The main difficulty for applying BSS techniques to extract the AA from Holter recordings is the lack of ECG leads. The number of observation required to solve a BSS problem is, at least, the number of mixed sources [14]. Given that AA, VA, noise and other bioelectric signals are always present in the ECG generation, two Holter leads are not enough to determine the AA. That is, the limited numbers of leads recorded from a Holter system reduces excessively the necessary spacial diversity required by BSS techniques to accurately extract the AA. Consequently, other extraction techniques diferent from BSS, as Average Beat Substrac- 
tion (ABS) [12], are commonly prefered when multi-lead ECG recordings are not available and individual leads are used instead [31]. Therefore, if convolutive BSS algorithms will be used to extract the AA from ECG Holter recordings of AF episodes, the number of observations must be increased. One way to solve this difficulty is including a wavelet decomposition of the Holter leads. The wavelet analysis can transform a signal under investigation into a set of signals so that a multiplied number of observations is made available. These new signals are called detail and approximation coefficients, each of them carrying information of different frequency bands of the ECG [119]. The inclusion of a wavelet decomposition stage to increase the number of leads was first introduced by Sanchez et al as the Wavelet Blind Separation (WBS) method [41]. Next, a new convolutive BSS algorithm optimized for the AA extraction from Holter recordings of AF episodes will be introduced. This new method is based on wavelet decomposition to increase the number of observations and will be subsequently referred as Convolutive Multiband Blind Separation (CMBS). In the next two subsections the wavelet transform principles will be summarized and the CMBS algorithm will be described.

\subsubsection{Wavelet Transform Principles}

Wavelet analysis is used to transform the signal under investigation into another representation that presents the signal information in a more useful form, joining spectral and temporal analysis [119]. Mathematically speaking, the Wavelet Transform (WT) is the convolution of the analyzed signal, $s(t)$, with a set of wavelet functions of a wavelet family, $\Psi_{a, b}(t)$. All these functions derive from a unique wavelet mother or basis wavelet, $\Psi(t)$ and are dilated and displaced versions of it. The Continuous Wavelet Transform (CWT) of $s(t)$ is provided by [119]:

$$
C_{a}(b)=\int_{-\infty}^{\infty} s(t) \Psi_{a, b}^{*}(t) d t
$$

where $a, b \in \Re, \quad a \neq 0$, are the scale and translation parameters, respectively, and $t$ is the time. The relation between the wavelet functions $\Psi_{a, b}(t)$ and their mother wavelet or basis function is:

$$
\Psi_{a, b}(t)=|a|^{-1 / 2} \Psi\left(\frac{t-b}{a}\right)
$$

As $a$ increases, the wavelet becomes narrower. Thus, one has a unique analytic pattern and its replications at different scales and with variable time localization.

The wavelet mother used to generate all the basis functions is based on some desired characteristics associated with that function. The translation parameter $b$ relates to the location of the wavelet function as it is shifted through the signal. Thus, it corresponds to the time information in the Wavelet Transform. The 
scale parameter $a$ is defined as $1 /$ frequency and is related to frequency information. Scaling either dilates (expands) or compresses a signal. Large scales (low frequencies) dilate the signal and provide global information about the signal, while small scales (high frequencies) compress the signal and provide detailed information hidden in the signal. The above analysis becomes very useful as in most practical applications, high frequencies (low scales) do not last for a long duration, but instead, appear as short bursts, while low frequencies (high scales) usually last for entire duration of the signal [120]. Given the varying time and frequency resolution at different scales, the wavelet analysis is also known as Multi-Resolution Analysis (MRA) [119]. An example of convolution of an arbitrary signal with two functions of the same wavelet family for two different scale factors ( $a=1$ and 5), and four different translation values of $b$ is displayed in figure 4.9.

When discrete functions are considered, the discrete wavelet transform (DWT) results from discretizing scale and translation parameters. Particularly, the definition of parameters as $a=2^{j}$ and $b=k \cdot 2^{j}$ leads to the dyadic DWT (DyWT), expressed as [119]:

$$
c(j, k)=\sum_{n \in Z} x[n] \Psi_{j, k}[n]
$$

where

$$
\Psi_{j, k}[n]=2^{-j / 2} \Psi\left[2^{-j} n-k\right]
$$

and

$$
a=2^{j} ; \quad b=k \cdot 2^{j} ; \quad(j, k) \in Z^{2}
$$

The implementation of the DyWT can be done by executing a hierarchical structure decomposition of the analyzed signal, so that the DyWT is computed as successive lowpass and highpass filtering of the discrete time-domain signal. This version of MRA is called the Mallat algorithm or Mallat-tree decomposition [121]. This algorithm is schematized in figure 4.10, where the low pass filter is denoted by $G 0$, while the high pass filter is denoted by $H 0$. At each level, the high pass filter produces detail information, $d[n]$, while the low pass filter associated with scaling function produces coarse approximations, $a[n]$.

The possibility of reconstruction of the original signal from some of the obtained basic blocks without loss of information is other important advantage of this discrete transforms. An approximation to the signal at scale $m$ can be generated by adding a wavelet of mother functions at this scaled factored by approximation coefficients as follows $[119,120]$ : 

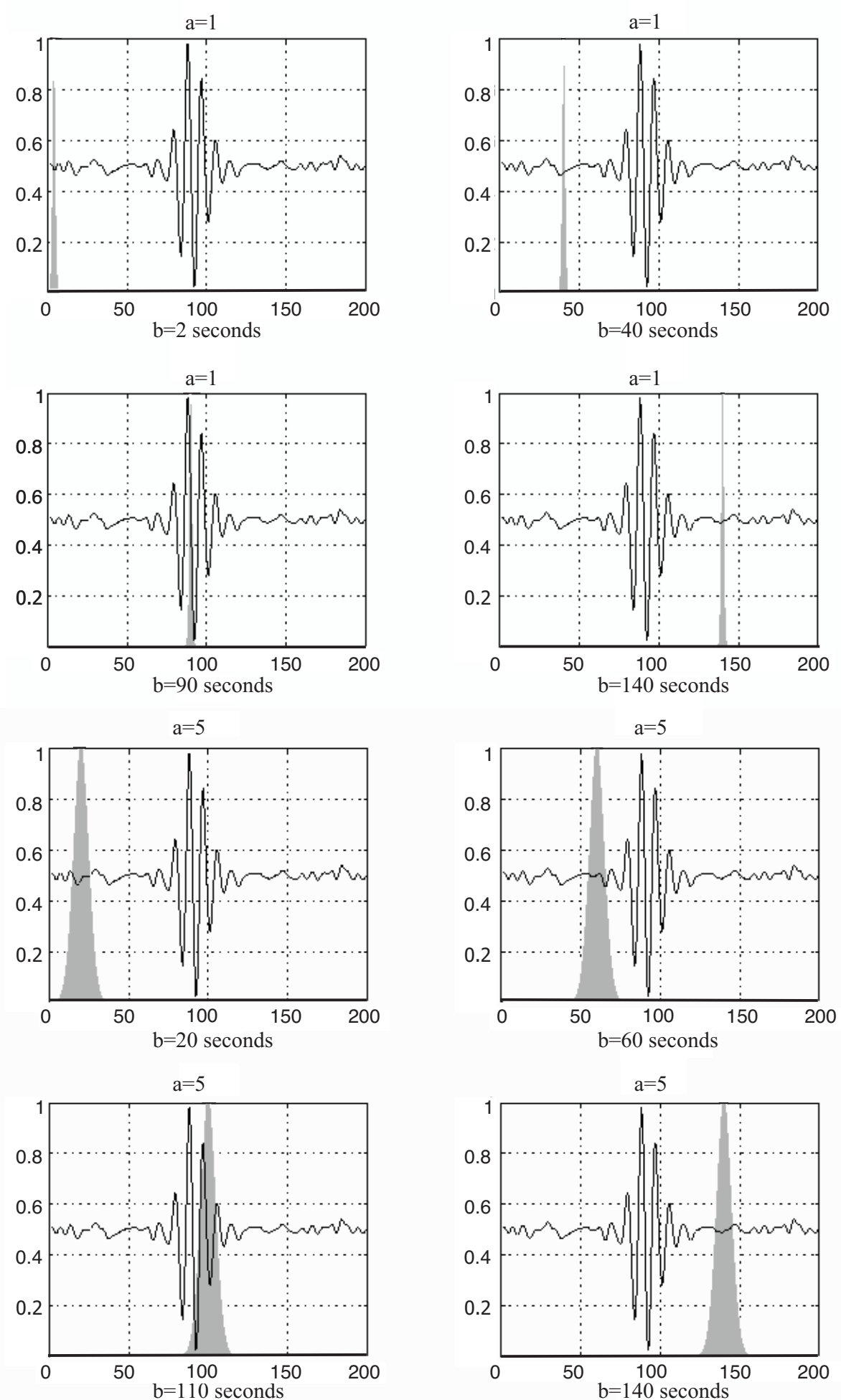

Figure 4.9. Example of convolution between an arbitrary signal and a wavelet function at two different scales, $a=1$ and $a=5$, and for different time shifts given by the translation parameter $b$. 


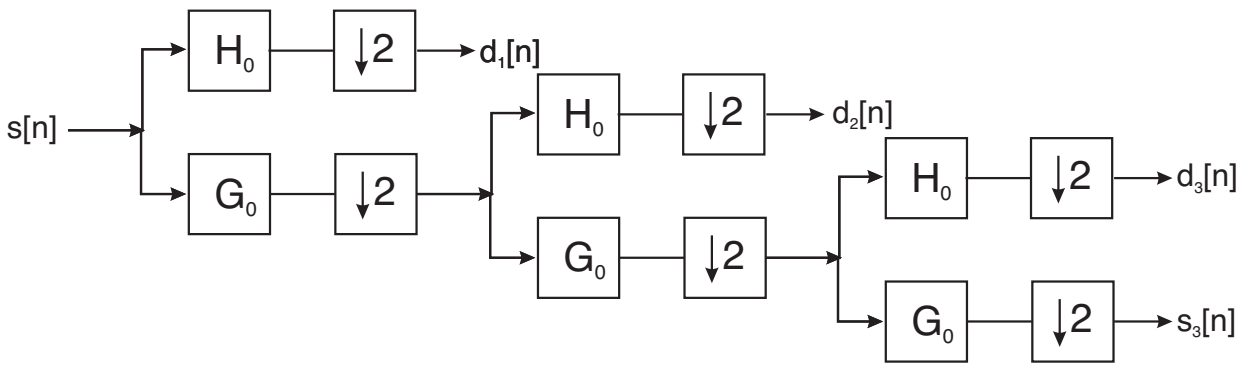

Figure 4.10. Multi-Resolution Analysis by successive lowpass and highpass filtering

$$
s_{m}(k)=\sum_{n} C_{m}(n) \cdot \Psi_{m, n}(k)
$$

and the original signal can be reconstructed back by adding its approximations at all scales. This mathematical synthesis is called the Inverse Discrete Wavelet Transform (IDWT) and has the next representation in the discrete domain[119, 120]:

$$
s(k)=\sum_{m} \sum_{n} C_{m}(n) \cdot \Psi_{m, n}(k)
$$

\subsubsection{A New Algorithm for Atrial Activity Estimation: Convolu- tive Multiband Blind Separation}

After describing the inherent problems of AA extraction from ECG Holter recordings, the CMBS algorithm can be already designed. It basically consists of wavelet decomposition stage plus a convolutive BSS stage. The general process of CMBS is schematized in figure 4.11. The Mallat-tree decomposition allows to increase the number of observations from Holter ECG recordings so that CBSS algorithms have enough entries to estimate the AA of AF episodes. Mathematically, if $x_{1}$ and $x_{2}$ are two leads of a Holter ECG recording, the wavelet stage will produce $N$ new signals from each lead that are observations inputs for the BSS stage, where $N$ is a natural number that depends on the level of wavelet decomposition. For instance, if the level of decomposition is six, $N$ is equal to seven since six detail and one approximation coefficients are considered. That is:

$$
\begin{aligned}
& x_{1} \longrightarrow\left[x_{11}, x_{12}, \ldots, x_{1 N}\right] \\
& x_{2} \longrightarrow\left[x_{21}, x_{22}, \ldots, x_{2 N}\right]
\end{aligned}
$$




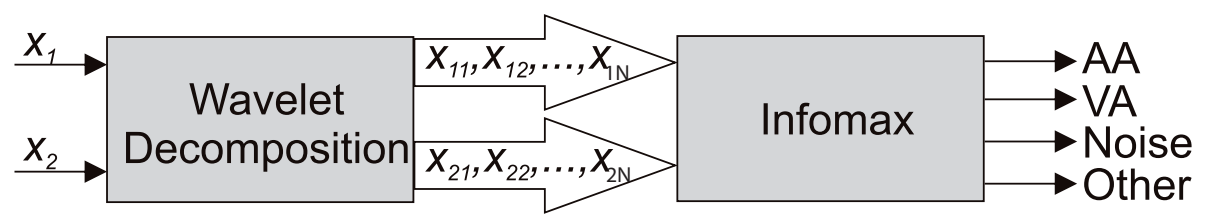

Figure 4.11. General CMBS process for AA extraction.

Hence the BSS stage must solve the following BSS problem:

$$
\left[\begin{array}{l}
x_{11} \\
x_{12} \\
\vdots \\
x_{1 N} \\
x_{21} \\
x_{22} \\
\vdots \\
x_{2 N}
\end{array}\right]=\mathbf{A} *\left[\begin{array}{l}
s_{1} \\
s_{2} \\
\vdots \\
s_{N} \\
s_{N+1} \\
x_{N+2} \\
\vdots \\
x_{2 \cdot N}
\end{array}\right]
$$

There still exist several unsolved questions of the algorithm design. Firstly, the optimal wavelet mother for the decomposition of Holter ECG recordings must be chosen. Secondly, the optimal level of decomposition for the selected wavelet mother must be selected too. The scheme of figure 4.11 is particularized for eight decomposition levels, and it can be anticipated that this value has not been arbitrarily chosen but is the result of a deep previous performance study of several wavelet mothers and decomposition levels. All the analyzed wavelet mothers belong to wavelet families that are available in Matlab code. The analyzed wavelet mothers and the decomposition levels at which they are used are detailed in Table 4.1. The symmlet(7), Daubechies(10), Symmlet(7) and Meyer wavelet mothers were used in [41] and [122], where the most suitable wavelet mother is adaptively selected in different fragments of the ECG according with the form of each QRS complex. Similarly, the wavelet mothers Haar, Daubechies(5), Coiflet(3), Biorthogonal (4.4), Reverse Biorthogonal(4.4) and Symmlet(5) were used in [123]. A detailed description of this functions can be found in [119]. Four of the studied wavelet mothers are depicted in Figure 4.12. The analyzed wavelet mothers and their levels of decomposition are presented in Table 4.1. A performance comparison of these wavelet mothers will be presented in chapter 7 and will allow to complete the algorithm design. The number of analyzed decomposition levels is different for every family because both the mother and the decomposition level were adjusted jointly and iteratively.

It can be anticipated too that the function symlet(8) with eight levels of decomposition was used as mother wavelet, given that it offered the best perfor- 


\begin{tabular}{l|l} 
Wavelet Family & Levels of Decomposition \\
\hline symmlet(6) & 5 and 6 \\
symmlet(7) & 5 to 7 \\
symmlet(8) & 5 to 8 \\
symmlet(9) & 5 to 9 \\
bior(4.4) & 7 and 8 \\
Daubechies(10) & 7 and 8 \\
Coiflet(3) & 8 \\
Harr(4) & 8 \\
\hline
\end{tabular}

Table 4.1. Analyzed wavelet mothers and their levels of decomposition.
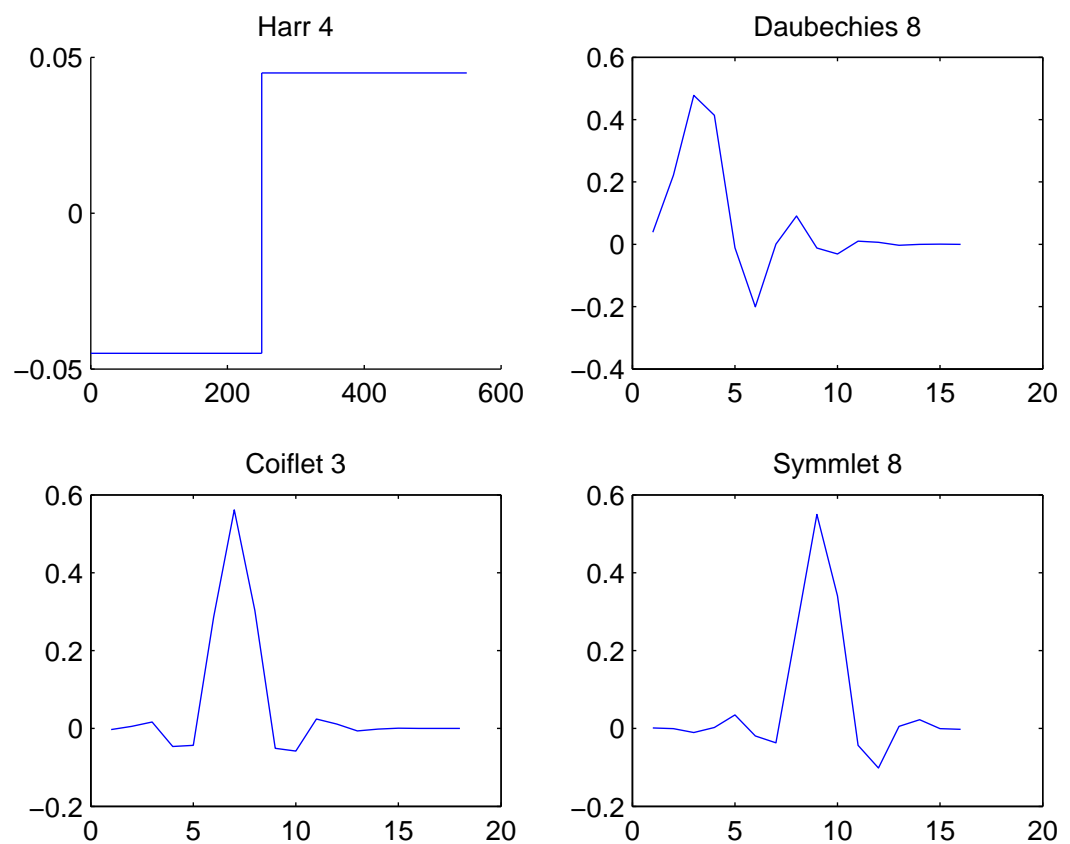

Figure 4.12. Several mother wavelet functions used to transform Holter leads into a higher number of observations 
mance among all the analyzed families. All detail coefficients and only one approximation coefficient of the lead decomposition stage are taken as inputs of the BSS stage. Thus nine observation signals resulted from each Holter lead. Consequently, eighteen signals were used as inputs of the BSS stage, as depicted in Figure 4.13. Note that the detail coefficients $d_{1}$ and $d_{2}$ do not contain significant information because of the spectral properties of Holter signals. Therefore, only seven useful wavelet signals from each lead are considered in practice. Hence the number of significant signals coming to the BSS stage is fourteen when two Holter leads are available.

The complete design of CMBS also requires to choose the optimal CBSS algorithm for AA extraction. It can be also anticipated that this optimal algorithm is the convolutive BSS algorithm Infomax [110], as will be corroborated by results given in chapter 7 . The results presented in chapter 7 will justify the use of a convolutive model as well.

Finally, a crucial decision is which one of the estimated sources $s_{1}, s_{2}, \ldots, s_{N}$ carries the information related to the AA. As for the before described convolutive BSS alogrithms (Infomax, MBLMS, TDD and CoBliSS), spectral analysis is used to identify the AA among the extracted signals. This will be explained in depth in chapter 6 . The selection of the AA from the sources estimated by convolutive Infomax is made regarding to the spectral morphology of the signals. The typical power spectrum of AA signals contains a sharp peak between 4 an $8 \mathrm{~Hz}$ and insignificant content in the rest of frequencies [124]. This selection can be made either by visual observation of the extracted sources spectra, or by using the Spectral Concentration $(S C)$ index, which automatizes the decision [93]. The $S C$ will be later defined in chapter 6 . 


Holter lead $(0$ to $500 \mathrm{~Hz})$

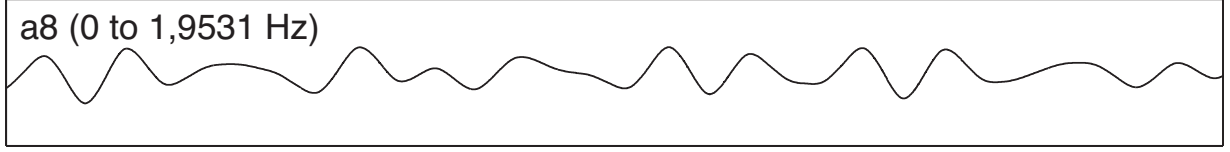

d8 (1,9531 to $3,9063 \mathrm{~Hz})$

d7 $(3,9063$ to $7,8125 \mathrm{~Hz})$

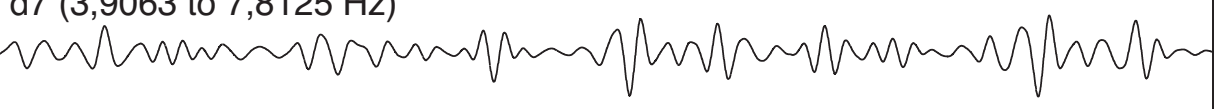

d6 $(7,8125$ to $15,625 \mathrm{~Hz})$

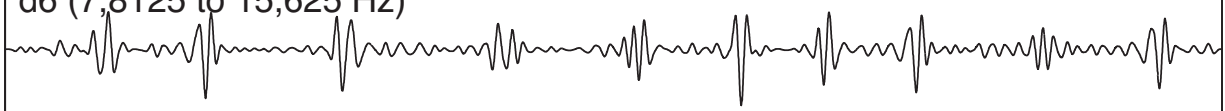

d5 (15,625 to $31,25 \mathrm{~Hz})$

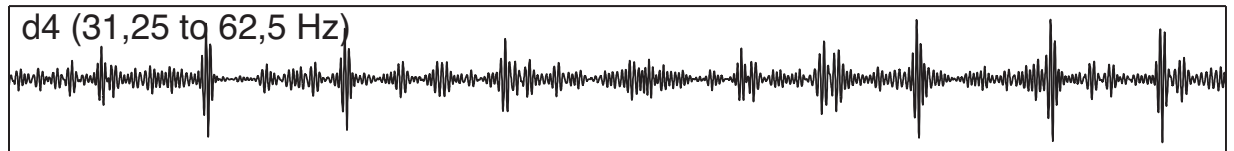

d3 $(62,5$ to $125 \mathrm{~Hz})$

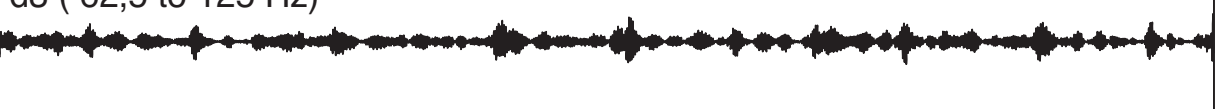

d2 $(125$ to $250 \mathrm{~Hz})$

d1 $(250$ to $500 \mathrm{~Hz})$

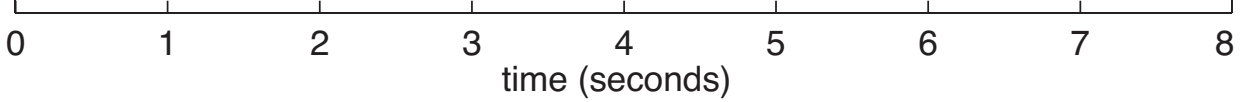

Figure 4.13. Decomposition of a Holter lead into 8 detail and one approximation coefficient by using symmlet 8 with 8 decomposition levels 


\section{Chapter 5 \\ Prediction of Paroxysmal Atrial Fibrillation Termination}

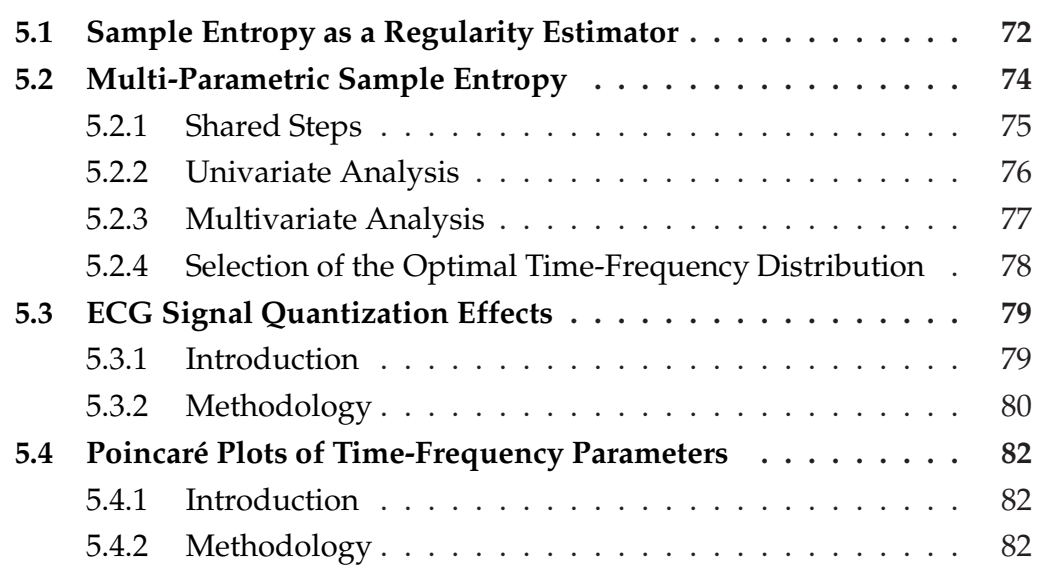

In this chapter, a new method based on the analysis of AA spectral features organization is presented with the aim to predict PAF episode termination. We have centered our work in disclosing the differences of spectral features organization between terminating and non-terminating PAF episodes. The analysis of spectral features is made in terms of mathematical regularity of their series. The organization is measured by using the entropy estimator Sample Entropy (SampEn) [43, 125]. This chapter is organized as follows. In section 5.1 the regularity estimator SampEn and its related nomenclature are introduced. A new method based on spectral features for predicting PAF termination is described in section 5.2. The effects of signals quantization on the suitability of spectral analysis are taken into consideration in section 5.3. Finally, a visual prediction method based on Poicaré plots that complements the analytic assessment of spectral features is presented in section 5.4. 


\subsection{Sample Entropy as a Regularity Estimator}

Given that the electrical remodeling present in the heart when AF occurs is a far-from linear process [91], the non-linear index SampEn was chosen to estimate the regularity of series. Other non-linear indices, as the Approximate Entropy $(A p E n)$ [125], which has been previously used in several biomedical applications [43], were also tested. Nevertheless, among the explored indexes, the SampEn was the one that achieved the higher percentage of correctly classified PAF episodes. The SampEn [43] measures the regularity level of series. That is, they quantify how predictable series are depending on the number of times that repetitive patterns are present in them. The $S a m p E n$ appears as a natural evolution of the Approximate Entropy $(A p E n)$ with the aim to reduce the bias of this estimator [43].

The main advantage of $S a m p E n$, in comparison with other nonlinear regularity indices, is its largely independency on the record length [43]. The SampEn operator explores numeric series in search of similar epochs and assigns a nonnegative number to the sequence. Large values of SampEn indicate high mathematical complexity or irregularity of data. Conversely, low values of SampEn indicate low complexity or high regularity of data. The computation of SampEn requires, besides the numeric series, two additional inputs: the length of patterns, $m$, and the tolerance window or criterion of similarity, $r$. The SampEn reflects the likelihood that similar patterns of observations will not be followed by additional similar observations. A time series containing many repetitive patterns has a relatively small SampEn. A less predictable, i.e. more complex, process has a higher SampEn. A major difference between SampEn and ApEn is that SampEn does not count self-matches and, consequently, has a reduced bias [43]. In a formal way, the SampEn is defined as follows:

Let $x[n]$ be a numeric series of length $N$ that consists of $N-m+1$ patterns $X_{m}(i), 1 \leq i \leq N-m+1$ of length $m$. That is:

$$
x[n]=\left[X_{m}(1), X_{m}(2), \ldots, X_{m}(N-m+1)\right]
$$

where

$$
X_{m}(i)=[x(i), x(2), \ldots, x(i+m-1)], \quad 1 \leq i \leq N-m+1
$$

The distance between any two patterns of the series, $X_{m}(i), X_{m}(j)$, of length $m$ is defined as:

$$
d\left[X_{m}(i), X_{m}(j)\right]=\max _{k=0, \ldots, m-1}(|x(i+k)-x(j+k)|)
$$

Let $B_{i}$ be the number of patterns of length $m$ that fulfill $d\left[X_{m}(i), X_{m}(j)\right]<r$ with $1 \leq j \leq N-m, j \neq i$, where $r$ is the feature that defines the criterion of similarity between patterns [125]. The fraction of patterns in $x[n]$ of length $m$ that 
resemble the pattern $X_{m}(i)$ is:

$$
B_{i}^{m}(r)=\frac{1}{N-m-1} B_{i}
$$

The mean of these $B_{i}^{m}(r)$ is the probability that two patterns in $x[n]$ of length $m$ accomplish that $d\left[X_{m}(i), X_{m}(j)\right]<r$ :

$$
B^{m}(r)=\frac{1}{N-m} \sum_{i=1}^{N-m} B_{i}^{m}(r)
$$

Similarly, considering $A_{i}$ as the number of patterns in $x[n]$ of length $m+1$ that fulfill $d\left[X_{m+1}(i), X_{m+1}(j)\right]<r$, with $1 \leq j \leq N-m, j \neq i, A_{i}^{m}(r)$ and $A^{m}(r)$ are defined as:

$$
\begin{gathered}
A_{i}^{m}(r)=\frac{1}{N-m-1} A_{i} \quad 1 \leq N-m \\
A^{m}(r)=\frac{1}{N-m} \sum_{i=1}^{N-m} A_{i}^{m}(r)
\end{gathered}
$$

where $A_{i}^{m}(r)$ is the probability that two patterns in $x[n]$ of length $m+1$ accomplish that $d\left[X_{m+1}(i), X_{m+1}(j)\right]<r$. Finally, the SampEn is defined as:

$$
\operatorname{Samp} \operatorname{En}(m, r)=\lim _{N \rightarrow \infty}\left\{-\ln \left[\frac{A^{m}(r)}{B^{m}(r)}\right]\right\}
$$

For a finite sequence of length $N$, its $\operatorname{SampEn}(m, r)$ is estimated by the statistic:

$$
\operatorname{SampEn}(m, r, N)=-\ln \left[\frac{A^{m}(r)}{B^{m}(r)}\right]
$$

The SampEn is usually computed for $m$ equal to 1 or 2, and $r$ between 0.1 and 0.25 times the standard deviation of the numeric sequence. This normalization produce good statistical validity of SampEn and makes it independent from any translation or scale invariance. In other words, the SampEn remains unchanged under uniform magnification or reduction processes and constant shift of values $[125,126]$. 


\subsection{Multi-Parametric Sample Entropy}

In this section we will describe the method to predict the evolution of PAF episodes. The differences of regularity in the time-frequency domain will be the key to distinguish between terminating and nonterminating PAF episodes. The regularity analysis is assessed by the non-linear regularity estimator SampEn. Given that we are interested in describing the AA properties in the time-frequency domain, some transformations on the Holter ECG recordings must be carried out before applying the SampEn estimator. An scheme of the proposed general process to classify PAF episodes can be seen in figure 5.1. This new method will be henceforth referred as Multi-Parametric Sample Entropy (MPSE). The MPSE is completed in six main steps: extraction of the AA, computation of the spectrogram, curve fitting, construction of spectral features series, SampEn computation, and the final classification. Next we will describe each of these steps. With reference to the last step, the classification can be assessed either by by using univariate analysis or discriminant analysis. This will define two different versions of the method depending on the type of classification. In other words, steps from one to five are common to both versions of the method and will be referred as shared steps, whilst step six, i.e. the final classification, will be different for each version.

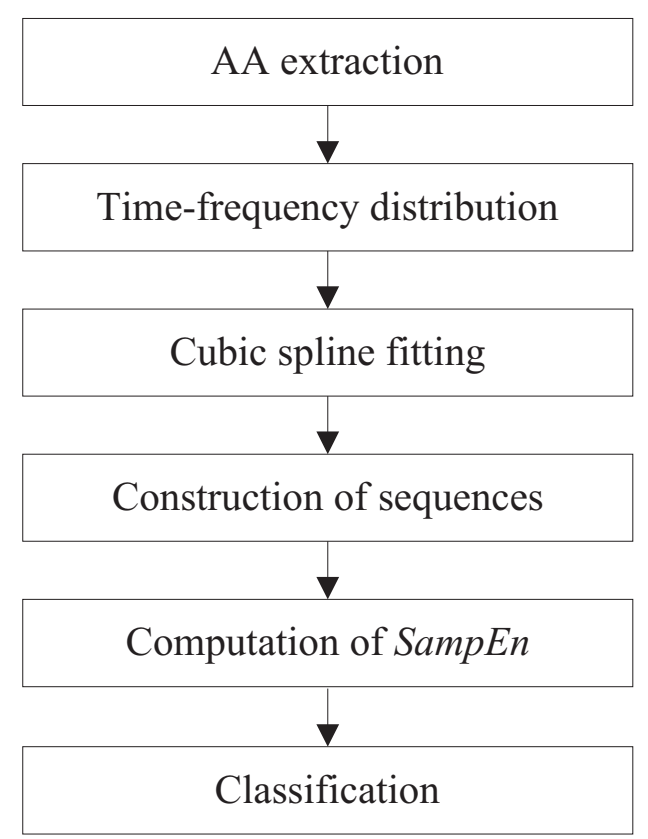

Figure 5.1. General process to classify a PAF episode as terminating or non-terminating 


\subsubsection{Shared Steps}

In order to use the ECG as a suitable tool for the analysis of AF, the AA must be separated from the other cardioelectric signals. The extraction of the AA during AF requires nonlinear signal processing since spectra of atrial and ventricular activities (VA) overlap and, in consequence, they cannot be separated by simple linear filtering [31]. In this sense, the CMBS method, which has been presented in chapter 4 , is fully optimized for the extraction of the AA when a reduced number of leads is available, as in Holter recordings, and a convolutive mixture of sources occurs, as it happens in the human body. Given that our database consist of Holter recordings, we have chosen the CMBS method to extract the AA as a previous step to the rest of the analysis. This selection will be fully justified in chapter 7.

After the extraction of the AA signal, its spectrogram [127] is computed with Hamming windows of 1024 samples in length and 75\% overlap. An example of AA spectrogram is depicted in $n$ Figure 5.2.a. How the spectrogram is chosen as the optimal time-frequency distribution to represent the AA in the timefrequency domain will be elucidated later in section 5.2.4. The results presented in chapter 7 will corroborate that the spectrogram is the best distribution for the study of AA regularity. Once the time-frequency distribution is computed, a cubic spline fitting is applied to each frequency slice that constitute the timefrequency distribution. This is exemplified in Figure 5.2.b for a spectrogram slice at $t=50$ seconds. In order to facilitate the spectral features extraction, the cubic spline fitting curve is interpolated so that the resulting frequency resolution is $0.01 \mathrm{~Hz}$. In this way, the spectral features of the AA are computed more accurately. The selection of the cubic spline model for spectral interpolation is justified because in several preliminary trials it provided the best fitting in comparison with Gaussian, polynomial, rational, Weibull, power and exponential models. A cubic spline is a piecewise function of third-order polynomials [128, 129]. Next, the local maxima and minima of the interpolated fitting curve are detected. Only singular points from the main peak to the end of the spectrum are taken into account in order to discard irrelevant maxima or minima. Several spectral features are then computed from these singular points. These spectral features will be introduced in subsection 6.4.2.

The next step consists of constructing the mathematical series of spectral features. Finally, the SampEn of all aforementioned series is computed in order to estimate their mathematical regularity. As it will be corroborated in chapter 7, some differences of signal regularity can be observed between terminating and non terminating episodes. The size of series is around 600 elements, what is large enough since the SampEn is meaningfully applied to more than 100 data points [125]. All SampEn values were computed with four significant digits. Given than the duration of the spectral features series is about a minute, the resultant series time resolution is 0.1 seconds. The values of $m=2$ and $r=0.25$ times the STD of signals were chosen for the computation of SampE, which are in the rank suggested by Pincus [126] $(m=1$ and $r$ between 0.1 and 0.25 times 
the STD). Other values of $m$ and $r$ were also evaluated, but the best results were reached with these values of $m$ and $r$. This selection will be later justified in chapter 7.

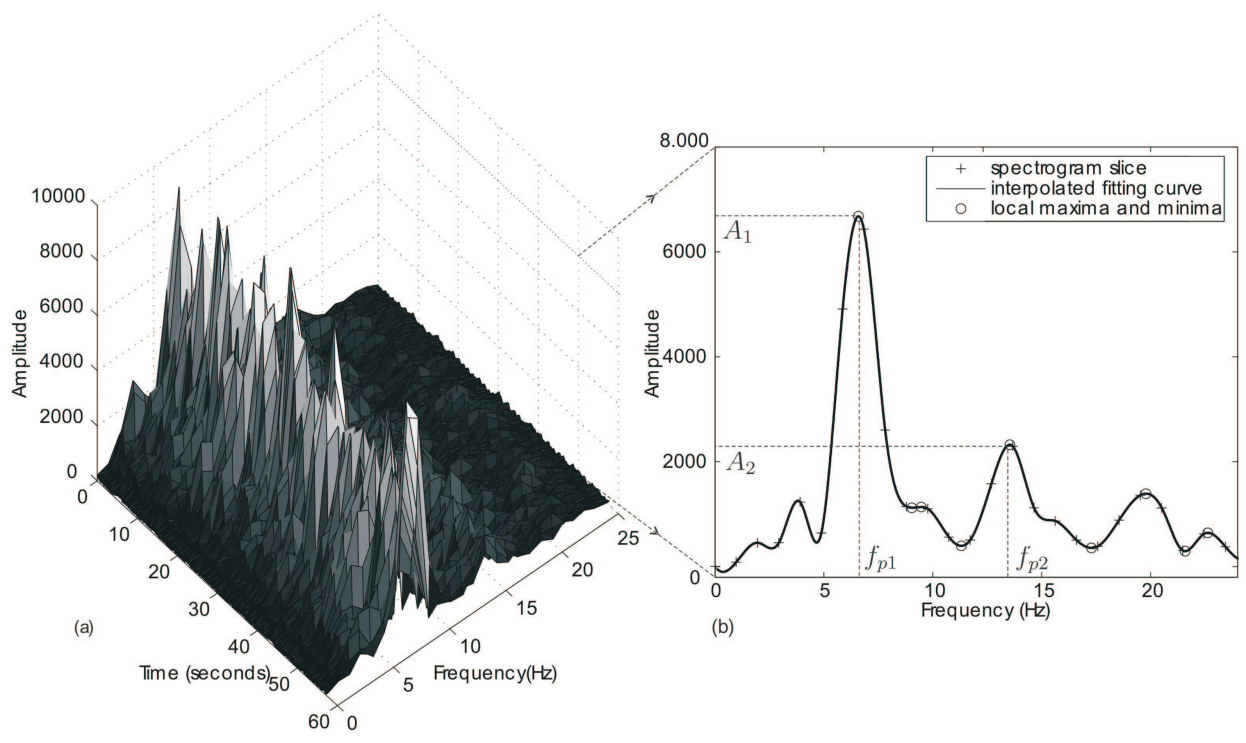

Figure 5.2. Time-frequency plot of a typical AA signal. a) AA spectrogram computed using Hamming windows of 1024 samples in length and 75\% overlap. b) Spectrogram slice at $t=50$ seconds, interpolated fitting curve, local maxima and minima, and spectral features $f_{p 1}, f_{p 2}, A_{1}$ and $A_{2}$.

\subsubsection{Univariate Analysis}

Once the SampEn of series is computed, we must be able to decide from this SampEn value whether the PAF episode is near to termination or, on the contrary, it is more likely to sustain. One straight choice for evaluating the SampEn values is fixing a threshold level of SampEn for every spectral feature. This threshold will be the limit between terminating and nonterminating PAF episodes. Since in this case the decision is made by regarding at only one variable each time, this type of decision is usually referred as univariate analysis. On the other hand, the complete procedure of the univariate analysis consists of two different phases that are highly related with the database structure, which will be introduced in chapter 6 . In a first stage, the threshold level must be fixed, which is carried out by considering a limited number of ECG recordings that constitute the so called learning set. After fixing the threshold level, which can be obviously different for every spectral feature, the suitability of the method is assessed by considering a different and independent group of recordings, which is referred as the test set. This procedure is depicted in Figure 5.3. In Figure 5.3.a the SampEn threshold level of a spectral feature is chosen by regarding at the learning set. Black circles indicate terminating episodes and white circles indicate nonterminating episodes 
This threshold is optimal since it provides the maximum number of correct classifications. Note that any displacement of this threshold up or down from its actual position would affect negatively to the total correct classifications. In Figure 5.3.b the recordings of the test set are classified by considering the threshold level previously fixed. The more correctly classified episodes of the test set the more suitable the classification method is.

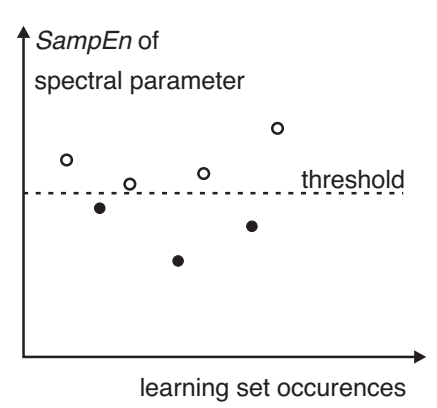

(a)

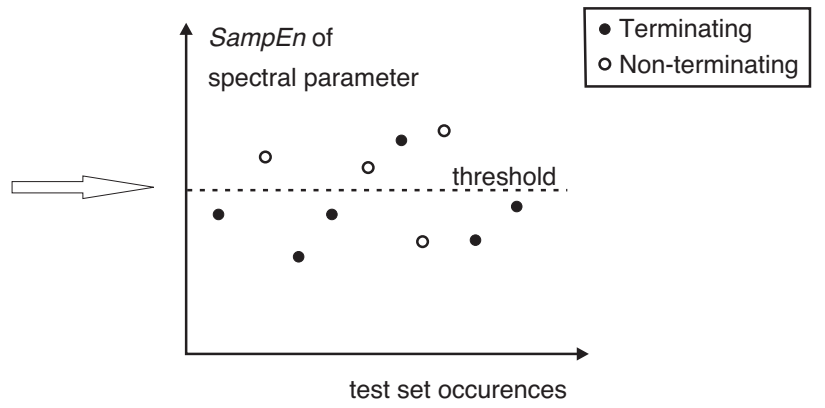

(b)

Figure 5.3. (a) The threshold of the univariate analysis is fixed as the optimal decision level of SampEn for the learning set (b) The method is finally assessed by considering the recordings of the test set and the threshold fixed by the learning set. Black circles indicate terminating episodes. White circles indicate nonterminating episodes.

\subsubsection{Multivariate Analysis}

In a univariate analysis the classification of a PAF episode as terminating or nonterminating is made by considering only a spectral feature at each time. Consequently, the final decision might be different for the same episode when different spectral feature are considered. In a multivariate analysis, several variables are considered at the same time so that the information carried by each variable is not regarded separately but all variables are jointly analyzed instead. Therefore, the final decision is not a function of a unique variable, but it is a function of several variables. In order to obtain this function of several variables we need to carry out a discriminant analysis where the SampEn of each spectral feature is taken into account. Thus we will subsequently refer to the multivariate analysis also as discriminant analysis. The objective pursued by the discriminant analysis is to know if there exists any combination of the aforementioned features which improves the decision reliability.

As in the univariate analysis, the discriminant analysis is carried out in two stages. First, the discriminant function is adjusted by considering the learning data set. This discriminant function is a linear transformation of the SampEn of spectral feature, which will be considered to be optimally adjusted when the maximum number of correctly classified learning PAF episodes is reached. Note that not necessarily all variables will be present in the final discriminant function because some carried information might be redundant. On the other hand, 
each variable present in the discriminant function will contribute it with a different weight. Then this discriminant function is assessed by the test set, as in the univariate case. An example of discriminant function of two input variables is depicted in Figure 5.4. Given that two variables contribute to the discriminant function, it is presented as a straight line. In Figure 5.4.a the multivariate function is adjusted from the recordings of the learning set. In Figure 5.4.b the multivariate function is assessed by the recordings of the test set. If three variables contributed to the discriminant function it would be a plane. More than three contributing variables implies a hyperplane as discriminant function.

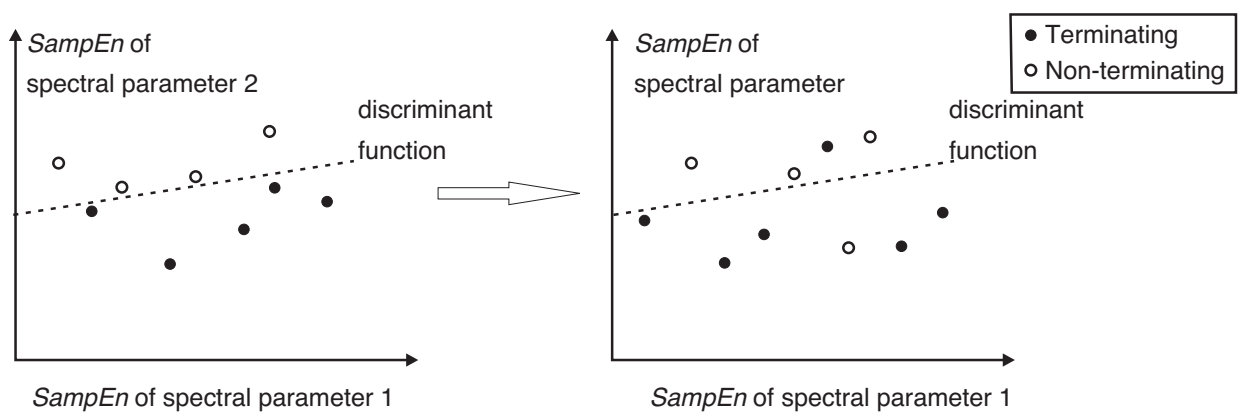

(a) Learning set

(b) Test set

Figure 5.4. (a) The discriminant function is adjusted from the learning set (b) The method is finally assessed by considering the recordings of the test set and the adjusted discriminant function. Black circles indicate terminating episodes. White circles indicate nonterminating episodes.

\subsubsection{Selection of the Optimal Time-Frequency Distribution}

Among the desirable properties of an energy time-frequency distribution, two of them are of particular importance : time and frequency covariance. Indeed, these properties guaranty that, if the signal is delayed in time and modulated, its time-frequency distribution is translated of the same quantities in the timefrequency plane. It has been shown that the class of energy time-frequency distributions verifying these covariance properties possesses the following general expression [127]:

$$
C_{x}(t, \omega)=\frac{1}{4 \pi} \iiint s^{*}\left(u-\frac{1}{2} \tau\right) s\left(u+\frac{1}{2} \tau\right) \Phi(\theta, \tau) \mathbf{e}^{-j \theta t-j \tau t+j \theta u} d u d \tau d \theta
$$

where $\Phi(\theta, \tau)$ is a two-dimensional function called the parameterization or kernel function. This class of distributions is known as the Cohen's class [127]. Several usual kernel functions are presented in table 5.1.

Although time-frequency distributions had been previously used in forecasting the evolution of AF episodes [23, 28], the SampEn is for the first time applied 


\begin{tabular}{cc} 
Name & Kernel: $\Phi(\theta, \tau)$ \\
\hline Wigner-Ville & 1 \\
Margenau-Hill & $\cos \frac{1}{2} \theta r$ \\
Page & $\mathbf{e}^{j \theta|\tau|}$ \\
Choi-Williams & $\mathbf{e}^{-\theta^{2} \tau^{2} / \sigma}$ \\
Spectrogram & $\int h^{*}\left(u-\frac{1}{2} \tau\right) \mathbf{e}^{-j \theta u} h\left(u+\frac{1}{2} \tau\right) d u$ \\
Zhao-Atlas-Marks & $g(\tau)|\tau| \frac{\sin a \theta r}{a \theta r}$
\end{tabular}

Table 5.1. Several usual kernel functions of time-frequncy distributions

to series of spectral features in the present work. Given that the optimal timefrequency distribution for the proposed method was a priori unknown, a preliminary study with the aim to choose it was advisable. This preliminary study was made by considering several time-frequency distributions and a reduced number of spectral features. The features chosen to do this prior survey were $f_{p 1}$ and $S C$. The $f_{p 1}$ was chosen because it is known from previous works that $f_{p 1}$ contains very relevant information on the AF termination $[22,23]$. The $S C$ was selected because it is a significant feature of real AF recordings [93]. Once the optimal time-frequency distribution was identified, the number of spectral features was extended to twelve.

The computed time-frequency distributions are those kernel functions are tabulated in Table 5.1 and other three derived distributions. All these time-frequency distributions belong to the Cohen's class $[127,130]$ and are the following: spectrogram (SP), Wigner-Ville (WV), pseudo-Wigner-Ville (PWV), Margenau-Hill (MH), pseudo-Margenau-Hill (PMH), Page (PG), pseudo-Page (PPG), Zhao-Atlas-Marks (ZAM) and Choi-Williams (CW). A in depth description of each of these distributions can be found in [127]. In order to use a unified criteria and avoid undesired sampling distortion on the selection of the optimal time-frequency distribution, all distributions were obtained with the same resolution of 1 second in time and $1 \mathrm{~Hz}$ in frequency. Results of this study will be shown in chapter 7 .

\subsection{ECG Signal Quantization Effects}

\subsubsection{Introduction}

In chapter 7 results will prove the suitability of MPSE for PAF termination prediction. In an ideal situation, our database would only consist of high resolution recordings. Nevertheless, these high resolution recordings are not always available in the regular clinical practice. Because of memory constraints of recorder devices, long duration recordings, as Holter systems, are normally sampled at a low frequency rate and with a low resolution, that is, the sampled values are 
stored by using a reduced number of bits. Since long duration recordings are frequently used as a diagnosis tool of AF, it would be interesting to know how Holter should be configured. In this section we are particularly concerned with the effects of quantization on MPSE. Here we present a methodology to assess the effects of ECG quantization on this PAF termination prediction method. We will analyze the effects of progressive decreasing signal resolution on several features used by MPSE.

\subsubsection{Methodology}

In order to analyze the quantization effects on MPSE, this study was particularized for two spectral features, namely the main peak frequency $\left(f_{p 1}\right)$ and the Spectral Concentration $(S C)$. On the other hand, the accuracy of the SampEn of the AA for different degrees of quantization is also computed. Hence, the effects of quantization on the estimation of signals regularity are also analyzed here. These features three will be later described in chapter 6 .

The general process is depicted in figure 5.5. The 16-bit original leads are quantized using from 15 to 4 bits. Then the CMBS algorithm is applied to extract the estimated AA from every signal. The digital post-BSS filter is a low-pass Chebyshev type II ninth order with cut-off frequency $70 \mathrm{~Hz}$ and $40 \mathrm{~dB}$ ripple in the stop-band and was applied to all the estimated AA signals . Next, the spectrograms of the filtered AA signals were computed using 1024 points Fast Fourier Transform (FFT), 1024 sample size Hamming window and $75 \%$ overlapping. Besides, sequences of the AA spectrograms characteristic features, i.e. the main peak frequency $\left(f_{p 1}\right)$ and the $S C$ ), were constructed. Furthermore, the $S a m p E n$ of quantized AA is also computed with the aim to measure the mathematical organization of time-domain signals $[43,125]$.

The results of this section will be presented in section 7.4. These results will allow us to make a recommendation on the minimum number of encoding bits. 
(a)

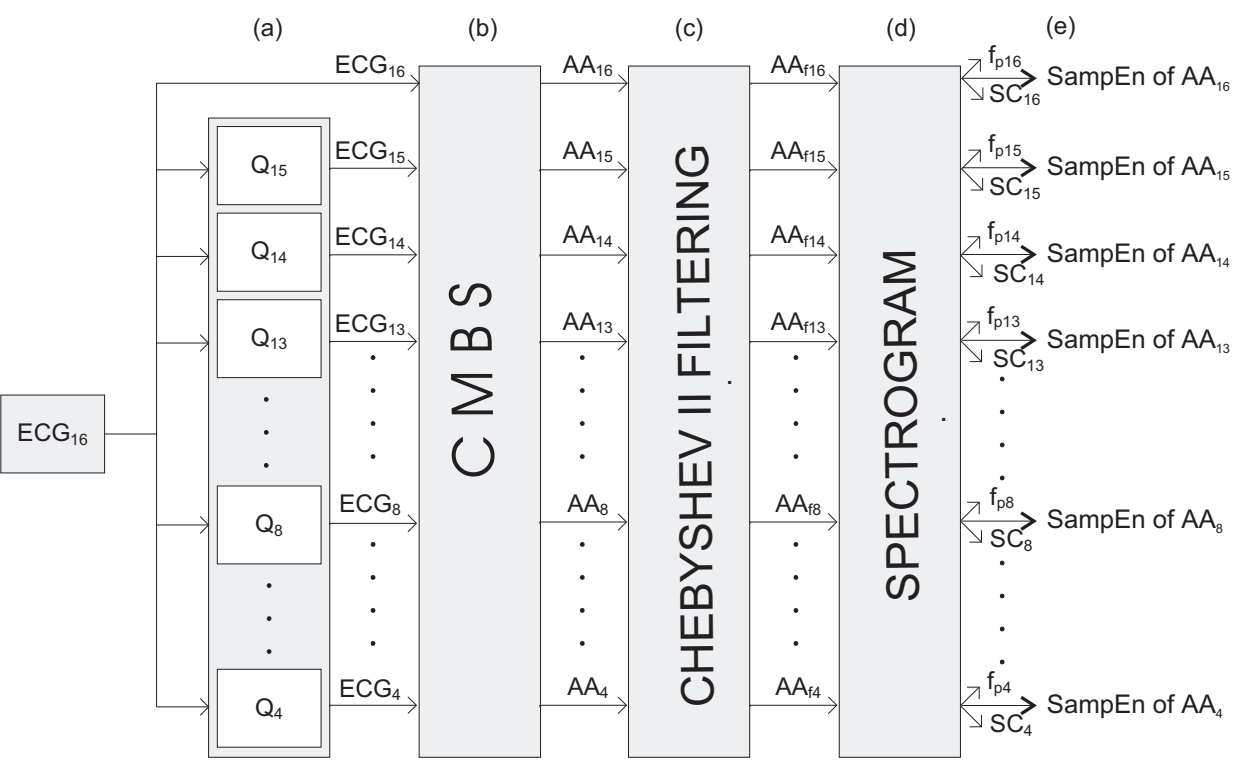

Figure 5.5. General process of analysis. Subindexes express the number of bits. (a) Quantization of leads, (b) AA extraction by the CMBS, (c) Low pass filtering, (d) Spectrogram of the quantized AA, (e) construction of $f_{p}$ and $S C$ sequences, and computation of the SampEn of AA. 


\subsection{Poincaré Plots of Time-Frequency Parameters}

\subsubsection{Introduction}

Given that in these critical situations a decision must be made with the utmost urgency, it would be desirable to have a visualization tool of easy interpretation that could provide a fast and reliable prediction of AF episode evolution. In this section, a new method to discriminate between terminating and nonterminating PAF episodes is presented. This new method is based on Poincaré plots and timefrequency analysis. The novelty of this method is the representation of timefrequency features on Poincaré $[131,132]$ plots as a new tool of decision. The main advantages of this new method are the low computational load and the visually easy interpretation of data.

\subsubsection{Methodology}

Firstly, the AA is extracted from the ECG recordings, given that the analysis of previously separated AA makes easier the study of AF [11] and improves the information provided by time-frequency distributions [37]. The AA activity is separated from the rest of cardioelectric sources by using the CMBS algorithm.

Secondly, the spectrograms of the extracted AA signals are computed using Hamming windows of 1024 samples in length and $75 \%$ overlap. In order to facilitate the extraction of spectrogram features, cubic spline fitting is applied to each of the Fourier transforms that comprises the spectrogram. The cubic spline model reached the best fitting in comparison with gaussian, polynomial, rational, Weibull, power and exponential models. The cubic spline fitting curve from the original data is interpolated so that the resulting frequency resolution is $0.01 \mathrm{~Hz}$ and the spectral features are hence extracted with higher accuracy. From four time-frequency features of every spectrogram we constructed four time-domain sequences. These features are the main peak frequency $\left(f_{p 1}\right)$, the second largest peak frequency $\left(f_{p 2}\right)$, and their respective peak magnitudes $\left(A_{1}\right.$ and $\left.A_{2}\right)$.

Finally, the phase portraits, i.e. the Poincaré maps with the stroboscopic view [131], of the aforementioned features were plotted. One phase portrait consists of plotting each data point of a periodically observed magnitude versus its predecessor [131, 132]). The formation of a stroboscopic view is exemplified in figure 5.6 for an arbitrary vector $x=\left[\begin{array}{lllllll}1 & 2 & 3 & 4 & 3 & 2 & 1\end{array}\right]$.

The visual inspection of clusters in the phase portraits, helped by the insertion of a cursor as a graphical threshold, was used to decide if the AF episode was of terminating or nonterminating nature. The results of this section will be presented in section 7.5 of chapter 7 , where the differences between stroboscopic views of terminating and nonterminating PAF episodes will be elucidated. The aforementioned overlap of $75 \%$ was necessary to have enough large numeric se- 


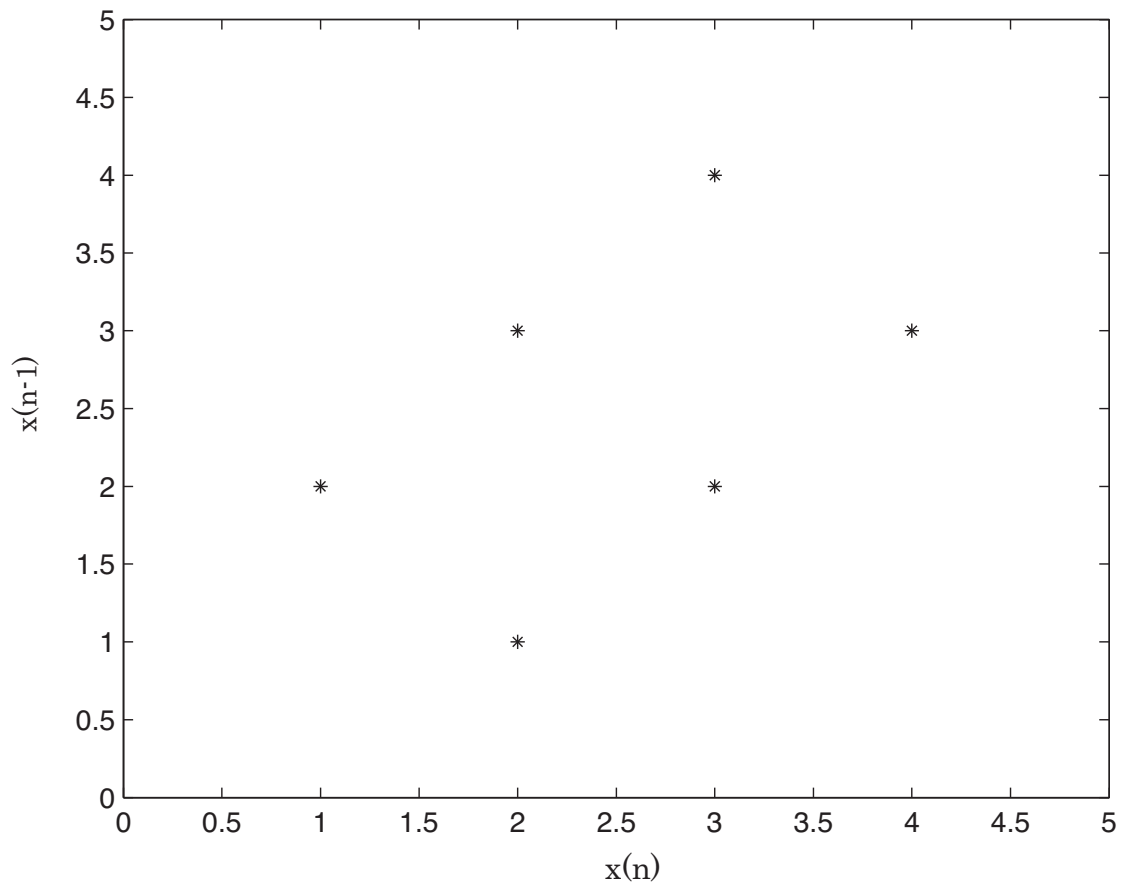

Figure 5.6. Example of the stroboscopic view of vector $x=\left[\begin{array}{lllllll}1 & 2 & 3 & 4 & 3 & 2 & 1\end{array}\right]$

quences, around 600 elements, so that the nature of PAF episodes could be deduced from the stroboscopic views. 



\section{Chapter 6 \\ Database and Performance Analysis}

6.1 Database Source and Signal Preprocessing ... . . . . . 86

6.2 Performance Analysis of CBSS Algorithms . . . . . . . 86

6.2 .1 Testing Environments . . . . . . . . . . . . . . . . . . 86

6.2 .2 Performance Indicators . . . . . . . . . . . . . . . . . . . . . . . . . . . . .

6.2 .3 Identification of the AA source . . . . . . . . . . . . . . . 94

6.3 Performance Analysis of CMBS . . . . . . . . . . . . . 94

6.3 .1 Testing environments . . . . . . . . . . . . . . . . . . . . 95

6.3 .2 Performance Indicators . . . . . . . . . . . . 96

6.4 Performance Analysis of AF Termination Prediction . . . . . 96

6.4.1 Testing Environment . . . . . . . . . . . . . . . . . . . . 97

6.4 .2 Spectral Features in MPSE . . . . . . . . . . . . 97

6.4.3 Performance Indicators of Quantization Effects . . . . . 98

In this chapter the employed database of signals and the indicators used for measuring the performance of methods are introduced. Furthermore, the use of performance indicators requires the definition of the appropriate testing environments. Each of the methods introduced in previous chapters will be assessed in one or two testing environments. This chapter is divided in three main subjects attending to the distribution of previous chapters: performance analysis of CBSS algorithms, performance analysis of CMBS, and performance analysis of AF termination prediction, which includes the analysis of MPSE, Poincaré plots, and quantization effects. 


\subsection{Database Source and Signal Preprocessing}

All signals in database are initially extracted from real ECG recordings. Several environments based on these original real recordings are defined in next sections. These original real signals are all preprocessed in order to reduce baseline wander, high frequency noise and powerline interference. The baseline wander is removed by bidirectional IIR Chebyshev high pass filtering with cut-off frequency of $0.5 \mathrm{~Hz}[133,134]$. The high frequency noise was reduced with an eight order bidirectional IIR Chebyshev low pass filtering with cut-off frequency of 70 $\mathrm{Hz}[135,136]$. The powerline interference is removed through adaptive filtering, which preserves ECG spectral information [137]. In addition, when the ECG recordings are registered at low frequency rate, they are upsampled to $1024 \mathrm{~Hz}$ with the aim to enhance the algorithms performance.

The initial real recordings come from two different sources. Firstly, twenty 12-leads ECG recordings of persistent AF episodes were made available by courtesy of the Clinic University Hospital of Valencia. These 12 recordings were captured at a frequency rate of $1 \mathrm{kHz}$ in the Cardiac Electrophysiology Laboratory of the aforementioned hospital by the diagnosis system Prucka Cardiolab 4000. An initial step previous to the study of CBSS algorithms performance is the the separation of AA and VA by the ABS algorithm so that they will be later used for the formation of synthetic ECGs. This will be later expanded in section 6.2 for each particular testing environment. Secondly, 50 real two-lead Holter recordings of PAF episodes were taken from the se of PhysioNet/Computers in Cardiology Challenge 2004. These signals were initially sampled at a frequency rate of 128 $\mathrm{Hz}$. A wider description of it will be given in section 6.4.1.

\subsection{Performance Analysis of CBSS Algorithms}

The performance analysis of CBSS algorithms comprises three main subjects: the description of the environments for testing the performance of algorithms, the performance indicators used to quantify the accuracy of the estimated AA, and the processes required to identify the AA among all the estimated sources. This three subjects are tackled in the next three subsections.

\subsubsection{Testing Environments}

In order to test the performance of convolutive BSS algorithms in the AA extraction two different test environments have been proposed. The signal database of each environment is constituted by synthetic ECG recordings. Nonetheless, there exist important differences between the synthesis process of both environments. The synthesis process of the first environment is a very controlled process so that 
all signal mixing features can be adjusted. This first environment does not represent a totally realistic mixture model but is very useful for the comparison of the AA extraction performance of convolutive BSS algorithms and the instantaneous algorithm FastICA. In a first step, the first environment is used to discard those convolutve algorithms which extraction performance is much lower than the performance of FastICA. By contrast, the synthesis process of the second environment is a more realistic one, since the resulting synthetic ECG recordings are effectively real ECG signals. This second environment provides a better approach to the analysis of real signals at the expense of less control of the synthesis process. Next, each testing environment is described separately.

\section{First Environment}

The signal database of the first environment consists of 15 pairs of separate AA and VA. The AA and the VA were previously extracted from real ECG recordings coming from the Cardiolab system by using the ABS algorithm. ABS was applied to lead V1 because this is the lead where the AA is more clearly distinguished. This extraction technique was chosen for this previous process because it is a widely used extraction technique which is based on QRST cancellation. Because of this, this previous process and the BSS techniques are made totally independent. In this way, this previous extraction process neither affects the performance study nor benefits the extraction performance of any algorithm under test BSS algorithm. All ECGs recordings are 10 seconds in length with a sampling rate of $1 \mathrm{kHz}$.

The separate AA and VA are are mixed by known linear functions in a controlled process. The synthesized ECG leads of AF are generated by combining the $\mathrm{AA}$ and the VA of the same AF episode by means of a randomized $2 \times 2$ mixing matrix A. The elements of the mixing matrix are FIR filters so that the mixture of $\mathrm{AA}$ and VA is made by regarding the mixture model given by equation 4.13 , that is, the convolutive mixture model. Henceforth, it will be said that these signals are mixed "convolutively". An example of synthetic leads formation from the original AA and VA can be seen in Figure 6.1 for a length of filters $h_{i j}$ equal to 2 . Attending to the size of matrix A, equation 4.13 can be rewritten as:

$$
\left[\begin{array}{l}
x_{1}[n] \\
x_{2}[n]
\end{array}\right]=\left[\begin{array}{ll}
h_{11} & h_{12} \\
h_{21} & h_{22}
\end{array}\right] *\left[\begin{array}{l}
s_{1}[n] \\
s_{2}[n]
\end{array}\right]
$$

where $s_{1}$ and $s_{2}$ are the mixed AA and VA, regardless of the appearing order, $x_{1}$ and $x_{2}$ are the synthetic leads, and $h_{i j}$ are FIR filters that constitute the mixing matrix $\mathbf{A}$. In the same way, 4.14 can be rewritten as:

$$
\left[\begin{array}{l}
\hat{s}_{1}[n] \\
\hat{s}_{2}[n]
\end{array}\right]=\left[\begin{array}{ll}
w_{11} & w_{12} \\
w_{21} & w_{22}
\end{array}\right] *\left[\begin{array}{l}
x_{1}[n] \\
x_{2}[n]
\end{array}\right]
$$




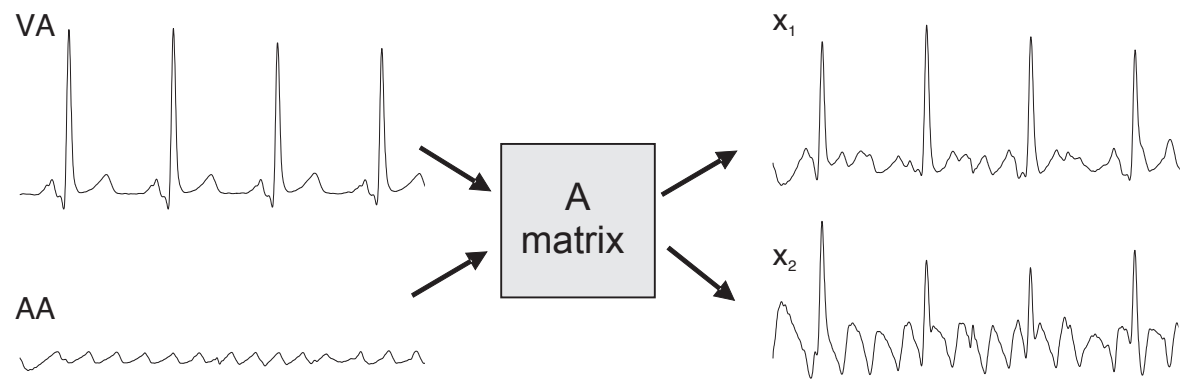

Figure 6.1. Example of synthetic leads $x_{1}$ and $x_{2}$ formation from the AA and the VA. The filters of matrix A take the following values in the example: $h_{11}=\left[\begin{array}{ll}1 & 0.5\end{array}\right], h_{12}=\left[\begin{array}{ll}2 & 1\end{array}\right], h_{21}=\left[\begin{array}{ll}1 & -0.5\end{array}\right]$, $h_{22}=\left[\begin{array}{ll}-2 & -1\end{array}\right]$.

where $\hat{s}_{1}$ and $\hat{s}_{2}$ represent the estimated AA and VA, regardless of the appearing order, and $w_{i j}$ are FIR filters that constitute the unmixing matrix $\mathbf{W}$.

In this context, the $h_{i j}$ filters length of equation 6.1 are varied in order to generate several types of convolutive mixtures, each of them contemplating a different maximum delay of sources. The length of $h_{i j}$ is represented by $N_{m}$ and has been changed from 1 to 8 . Consequently, the maximum considered signal delay of convolutive mixing is equal to 8 miliseconds, given that the sampling rate is $1 \mathrm{kHz}$. The selection of 8 as the maximum value of $N_{m}$ is justified because the delay between the QRS maxima of two arbitrary ECG leads is around a few miliseconds. Consequently, eight is a reasonable maximum value of the FIR filters length. Note that the instantaneous mixture is also taken into account in this environment, given that equation 4.13 matches equation 4.6 when $N_{m}$ is equal to one. Every value of $N_{m}$ defines a different experiment which results will be presented in chapter 7. As ten different mixing matrices are randomized for every $N_{m}, 150$ mixtures are generated for each experiment, seeing that 15 pairs of AA and VA signals are available.

In the separation process, the length of $w_{i j}$ filters is a feature that must be adjusted in all the tested CBSS algorithms. This new feature is referred as $N_{s}$ and is varied in each of the experiments. CoBliSS is the only tested CBSS algorithm that allows to adjust $N_{s}$ equal to one. In this case, the values $N_{s}=1,2,4,8,16$ and 32 were considered. In the rest of the algorithms, the lowest allowed value of $N_{s}$ is two, thus the values $N=2,4,8,16$ and 32 were considered instead. That is, the minimum of $N_{s}, 1$ or 2, is a constraint of every tested CBSS algorithm. On the other hand, the maximum of $N_{s}$, i.e. 32, was chosen so that the maximum controlled delay due to the mixing-unmixing process equals to $40 \mathrm{~ms}$ in duration, accordingly to the aforementioned maximum value of $N_{s}$ [97] and the sampling rate of $1 \mathrm{kHz}$. The tested lengths of $N_{m}$ and $N_{s}$ are shown in table 6.1. Not all values of the $N_{s}$ rank are tested but only those which are powers of two. The use of these $N_{s}$ values guarantees an optimal efficiency of the tested CBSS algorithms with regard to time execution, given that that all algorithms under test work in 
the frequency domain and are based on Fast Fourier Transform (FFT) computation.

\begin{tabular}{c|c|c} 
& CoBliSS & Rest of CBSS algorithms \\
\hline$N_{m}$ & $1,2,4,8$ & $1,2,4,8$ \\
\hline$N_{s}$ & $1,2,4,8,16,32$ & $2,4,8,16,32$ \\
\hline
\end{tabular}

Table 6.1. Tested filter lengths of the mixing and separation matrices in the first environment. $N_{m}$ is the length of the mixing matrix filters. $N_{s}$ is the length of the separation matrix filters.

In order to ensure the good working and utility of this first environment for testing BSS algorithm, several constraints were imposed on the generation of randomized matrices. The first restriction on the matrix randomizing is the invertibility of mixing matrices. That is, it must be accomplished the condition given by equation 4.21 , in the case of instantaneous mixture, or equation 4.23 , in the case of convolutive mixture. Furthermore, we require that FIR filters of the mixing matrix are minimum phase filters, since most of convolutive BSS algorithms are based on the neuromimetic Hérault-Jutten network and one restriction of this architecture is that FIR filters must have minimum phase [98]. This assumption is easily applicable to the mixture of biomedical signals in the human body, given that it is reasonable to think that the FIR filters are casual and stable, so that they obey the condition of minimum phase [14].

\section{Second Environment}

In the second environment, 12-leads ECGs of AF episodes are synthesized adding AA and VA of every lead. As in the first environment, the contributions to AA and VA of each lead were previously separated from real ECGs of AF episodes coming from the Cardiolab system by using QRST cancellation. The main difference with the first environment is that the synthetic ECG matches exactly the real ECG. In fact, the synthesized ECG is a reconstruction of the original ECG from the previously separated AA and VA of each lead. Other important difference from the first environment is the fact that now the mixing matrix is unknown. Although we know the AA and VA contributions on each lead, on the contrary, we do not know the mixture process nor the original AA and VA sources. Consequently, the mixing matrix of sources remains hidden. Therefore, this second environment is more realistic than the first one at expense of some control loss of the mixture process. This second environment comprises 20 synthetic ECGs. This process is depicted in Fig. 6.2 and can be mathematically expressed as [93]:

$$
\mathbf{x}_{\mathbf{i}}=\mathbf{x}_{\mathbf{i} A A}+\mathbf{x}_{\mathbf{i} A V},, \quad i=1, \ldots, 12
$$

where and $\mathbf{x}_{\mathbf{i}_{A A}}$ and $\mathbf{x}_{\mathbf{i}_{A V}}$ are the atrial and ventricular contribution of the $\mathrm{i}$-th lead respectively, and $x_{i}$ is the $i$-th synthesized lead. All resulting ECG recordings 


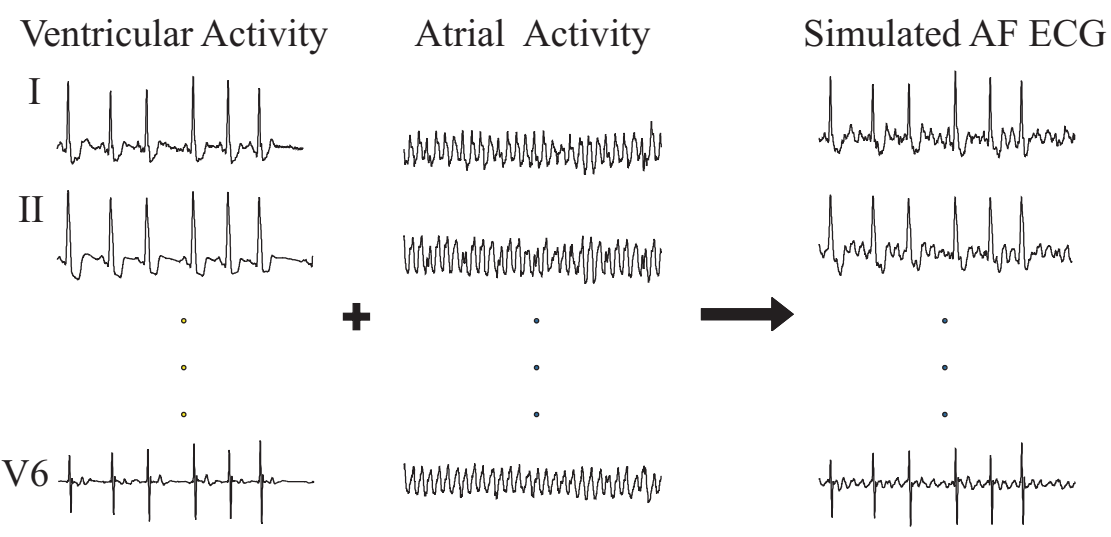

Figure 6.2. Second environment synthetic ECG generation. The AF 12-leads ECGs are synthesized by adding the AA and the VA of every lead, previously separated from real ECGs of AF episodes.

last for 10 seconds and are sampled at $1 \mathrm{KHz}$. This second environment comprises 20 synthetic ECGs.

With reference to the filter length of the mixing and unmixing matrices, the feature $N_{m}$ is unknown as well as the mixing process. On the contrary, the feature $N_{s}$ can still be fixed previously to the application of each CBSS algorithm. Since convolutive mixtures of bioelectric signals in the human body is assumed and the original mixing matrix $\mathbf{A}$ is unknown, we cannot determine the exact length of mixing matrix FIR filters in the second environment. In this second environment, $N_{s}$ varies from 1 to 128 or from 2 to 128 , depending on the CBSS algorithm. Not all values of the $N_{s}$ rank are tested but only those which are powers of two with the aim to facilitate the FFT computation.

\subsubsection{Performance Indicators}

For the study of the AA extraction performance reached by CBSS algorithms, two indicators have been employed, namely the Signal to Interference Ratio (SIR) and the index of cross-correlation between signals. Next both indicators are introduced along with the necessary adaptation to each testing environment.

\section{Signal to Interference Ratio (SIR)}

In an ideal separation process, the estimated sources match exactly the original sources. On the contrary, in a real separation process, the estimated sources are always contaminated by contributions of other sources to a certain extent. The SIR indicator measures this level of interference among the estimated sources as a ratio of signal powers. In a signal generation model of synthetic signals where both the original sources $s_{j}$ and the mixing matrix are known, as in the first en- 
vironment, this ratio can be easily computed. Nonetheless, our major interest is to know how CBSS algorithms improve the source separation. Therefore, the SIR must be computed before and after the application of BSS algorithms and then compared. This will define a new indicator that measures this improvement. Since our particular concern is the extraction of the AA, this indicator will be referred as $S I R_{A A}$, which measures the AA extraction performance as the increase of the SIR accomplished by BSS algorithms. A similar indicator for audio mixtures is defined in [138].

First, let us consider $x_{i}$ as the observation with the highest contribution of AA. The SIR ratio of $x_{i}$ is defined as [106, 138]:

$$
S I R_{A A}^{o}=10 \log \frac{\mathbf{E}\left\{\left(h_{i j} * s_{j}\right)^{2}\right\}}{\mathbf{E}\left\{\left(\sum_{\substack{k=1 \\ k \neq j}}^{N} h_{i k} * s_{k}\right)^{2}\right\}}
$$

where $h_{i j}$ are the FIR filters of the A mixing matrix, $*$ is the convolution operator, and the superscript $o$ stands for observed signal. In the same way, let us consider that $\hat{s_{p}}$ is the estimated source with the highest contribution of AA. The SIR ratio of $\hat{s_{p}}$ is $[106,138]$ :

$$
S I R_{A A}^{e}=10 \log \frac{\mathbf{E}\left\{\left(g_{p j} * s_{j}\right)^{2}\right\}}{\mathbf{E}\left\{\left(\sum_{\substack{k=1 \\ k \neq p}}^{N} g_{p k} * s_{k}\right)^{2}\right\}}
$$

where $g_{p k}$ are the FIR filters of the $\mathbf{G}$ global system matrix so that $\mathbf{G}=\mathbf{W} * \mathbf{A}$, $\mathbf{W}$ is the estimated separation matrix, and the superscript $e$ stands for estimated signal. Finally, the use of logarithmic units lead us to express $S I R_{A A}$ as:

$$
S I R_{A A}=S I R_{A A}^{e}-S I R_{A A}^{o}
$$

Now, let us consider the particular case of two sources and two observations of the first environment. In this case, since the matrix $\mathbf{A}$ is of size $2 \times 2$, the equation 4.13 , that describes the convolutive mixtures, can be rewritten as:

$$
\begin{aligned}
& x_{1}=h_{11} * s_{1}+h_{12} * s_{2} \\
& x_{2}=h_{21} * s_{1}+h_{22} * s_{2}
\end{aligned}
$$

In the same way, since the separation matrix is also of size $2 \times 2$, the equation 4.14, that describes the estimation of sources in the convolutive model, can be rewritten as: 


$$
\begin{aligned}
& \hat{s}_{1}=w_{11} * x_{1}+w_{12} * x_{2} \\
& \hat{s}_{2}=w_{11} * x_{1}+w_{12} * x_{2}
\end{aligned}
$$

The substitution of 6.7 into 6.8 yields:

$$
\begin{aligned}
& \hat{s}_{1}=w_{11} *\left(h_{11} * s_{1}+h_{12} * s_{2}\right)+w_{12} *\left(h_{21} * s_{1}+h_{22} * s_{2}\right) \\
& \hat{s}_{2}=w_{11} *\left(h_{11} * s_{1}+h_{12} * s_{2}\right)+w_{12} *\left(h_{21} * s_{1}+h_{22} * s_{2}\right)
\end{aligned}
$$

From equation 6.9, the $S I R_{1}^{o}$ can already be computed, seeing that all terms are known:

$$
S I R_{1}^{o}=10 \log \frac{\mathbf{E}\left\{\left(h_{11} * s_{1}\right)^{2}\right\}}{\mathbf{E}\left\{\left(h_{12} * s_{2}\right)^{2}\right\}}
$$

And the separation level of source $s_{1}$ in the estimated source $\hat{s_{1}}$ as:

$$
S I R_{1}^{e}=10 \log \frac{\mathbf{E}\left\{\left(w_{11} * h_{11} * s_{1}+w_{12} * h_{21} * s_{1}\right)^{2}\right\}}{\mathbf{E}\left\{\left(w_{11} * h_{12} * s_{2}+w_{12} * h_{22} * s_{2}\right)^{2}\right\}}
$$

Now the $S I R_{A A}$ is figured out by substituting equations 6.10 and 6.11 into equation 6.6. Note that, depending on the mixing matrix the subscripts of the source and the observed signal may not coincide.

In the second environment the mixing matrix is undefined as well as the original AA and VA sources. Only the partial contributions of AA and VA on every ECG lead are known. Despite this, the $S I R_{A A}$ defined in equation 6.6 can still be computed. In order to do this, the source and the observation with the highest contribution of AA are used for $S I R_{A A}$ computation. Let us assume that the lead with the greatest atrial activity contribution is V1. Therefore, $S I R_{A A}^{o}$ can be expressed as:

$$
S I R_{A A}^{o}=10 \log \frac{\mathbf{E}\left\{\left(x_{A A}^{V 1}\right)^{2}\right\}}{\mathbf{E}\left\{\left(x_{V A}^{V 1}\right)^{2}\right\}}
$$

When CBSS algorithms are applied, an unmixing matrix $\mathbf{W}$ is approximated so that the estimated atrial activity source can be expressed as:

$$
\hat{\mathbf{s}}=\mathbf{W} * \mathbf{x}
$$

Substituting equation 6.3 in 6.13 yields

$$
\hat{\mathbf{s}}=\mathbf{W} * \mathbf{x}_{A A}+\mathbf{W} * \mathbf{x}_{V A}
$$

Defining wAA $_{\text {A }}$ as the A row that provides the estimated atrial activity, the estimated AA signal, $\hat{s}_{A A}$, can be computed as: 


$$
\hat{s}_{A A}=\mathbf{w}_{A A} * \mathbf{x}
$$

Hence, the estimated atrial activity source, $\hat{s}_{A A}$, has a contribution of the original atrial activity signals, $s_{A A}$, and a spurious contribution of the VA signals, $s_{V A}$ :

$$
\hat{s}_{A A}=s_{A A}+s_{V A}=\mathbf{w}_{A A} * \mathbf{x}_{A A}+\mathbf{w}_{A A} * \mathbf{x}_{V A}
$$

where all terms are known. Then, $S I R_{A A}^{e}$ can be expressed as

$$
S I R_{A A}^{e}=10 \log \frac{\mathbf{E}\left\{\left(s_{A A}\right)^{2}\right\}}{\mathbf{E}\left\{\left(s_{V A}\right)^{2}\right\}}
$$

Finally, $S I R_{A A}$ is computed by using equation 6.6.

\section{Cross-correlation Index}

We define the cross-correlation index between two signals $x_{i}$ and $x_{j}$ as

$$
R_{i j}=\frac{\mathbf{E}\left\{x_{i} \cdot x_{j}\right\}}{\sqrt{\mathbf{E}\left\{x_{i}^{2}\right\} \mathbf{E}\left\{x_{j}^{2}\right\}}}
$$

which ranks between 0 and 1 . The nearer $R_{i j}$ to one the more similar the signals are. Conversely, the nearer $R_{i j}$ to 0 the more different the signals are.

Since we are interested in measuring the quality of AA extraction, for simplicity we will use in the future the following notation:

- $s_{A A}$ as the original atrial activity.

- $\hat{s}_{A A}$ as the estimated atrial activity.

- $R_{A A_{t}}$ as the cross-correlation between the original AA and the estimated AA. The subscript $t$ denotes that this cross-correlation index is computed in the time domain.

Hence equation 6.18 can be rewritten as:

$$
R_{A A t}=\frac{\mathbf{E}\left\{s_{A A} \cdot \hat{s}_{A A}\right\}}{\sqrt{\mathbf{E}\left\{s_{A A}^{2}\right\} \mathbf{E}\left\{\hat{s}_{A A}^{2}\right\}}}
$$

$R_{A A t}$ will be used, in addition to $S I R_{A A}$, to assess performance of CBSS algorithms in the AA extraction from AF episodes. 


\subsubsection{Identification of the AA source}

Considering that the typical spectral morphology for AA is characterized by a very pronounced peak in frequencies from 4 to $8 \mathrm{~Hz}$, with no harmonics and insignificant amplitudes above $15 \mathrm{~Hz}$ [139], it is possible to define a performance extraction index capable of evaluating AA extraction quality based on the spectral concentration. The spectral concentration $(S C)$ index is defined as [93]:

$$
S C=\frac{\sum_{f=0.82 f_{p 1}}^{1.17 f_{p 1}} P_{A A}(f)}{\sum_{f=0}^{0.5 f_{s}} P_{A A}(f)}
$$

where $P_{A A}$ is the power spectral density of the AA signal, $f$ is the independent variable of frequencies, $f_{s}$ is the sampling rate, and $f_{p 1}$ is the main frequency peak of the AA. Experimental observations prove that the AA is the estimated independent component with the highest $S C$ when the limits of the numerator summation are those specified in equation (6.20) [124]. Therefore, the $S C$ can be used as an indicator to select, in an automated way, the estimated source that best matches the AA. A wider distance between frequency limits introduces high frequency noise components in the calculation of the $S C$ indicator so that the probability of discriminating correctly the AA is reduced. A shorter distance between limits reduces the AA power considered in $S C$ so that the probability of AA discrimination is reduced too. The bandwidth considered for the SC computation is of $2 \mathrm{~Hz}$ for a typical $f_{p 1}$ of $6 \mathrm{~Hz}$, what justifies the selection of the lower and upper limits of the sum [93].

An additional strategy to identify the AA is the use of the fourth order cumulant, i.e. kurtosis, since the source separated from an AF episode that has the lower kurtosis is considered as the AA [11]. The kurtosis of a signal $y$ is defined as:

$$
\operatorname{kurt}(y)=\mathbf{E}\left\{y^{4}\right\}-3\left(\mathbf{E}\left\{y^{2}\right\}\right)^{2}
$$

\subsection{Performance Analysis of CMBS}

Two matters will be treated in the two following subsections. Firstly, the two environments where the CMBS algorithm is tested and compared with other extraction methods are described. Secondly, the performance indicators employed for the quantification of AA extraction performance are portrayed. 


\subsubsection{Testing environments}

As for CBSS algorithms, two different environments were proposed to test the AA extraction performance of CMBS. The first environment is a controlled mixture model. The second environment is more realistic but has less control on the mixture model. In order to study the effect of noise on the extraction performance of CMBS, tree levels of Additive White Gaussian Noise (AWGN) were considered in both scenarios: no noise, a Signal to Noise Ratio (SNR) of $5 \mathrm{~dB}$, and a SNR of $15 \mathrm{~dB}$. The SNR is defined as [140]:

$$
S N R=10 \log \left(\frac{\mathbf{E}\left(x^{2}[n]\right.}{\mathbf{E}\left(w^{2}[n]\right.}\right)
$$

where $x[n]$ is a free of noise signal and $w^{2}[n]$ is the AWGN added to $x[n]$.

\section{First Environment}

This environment is similar to the first environment described for the test of CBSS algorithms, given that the same 15 pairs of AA and VA signals are used here. The AA and VA are separated from each recording lead by ABS before the application of CMBS. Also the AA and VA are mixed convolutively by random matrices. Nonetheless, there exist some differences in the mixing and unmixing process. First, the length of the mixing matrix filters, $N_{m}$, is not varied now but is fixed to one. The reason is that now our objective is not the performance comparison of several CBSS algorithms. By contrast, we are concerned about the comparison of the best CMBS algorithm with BSS algorithms that follow the instantaneous mixture model, as FastICA. Since $N_{m}=1$ indicates the instantaneous model, this filter length is optimal for the comparison. Furthermore, CMBS is based in the Infomax algorithm, which optimizes results when $N_{m}$ equals one. These results for Infomax will be later detailed in chapter 7.

Another difference with the first environment for CBSS testing relates to the the length of the unmixing matrix filters, $N_{s}$. For similar reasons, the length of the unmixing matrix filters, $N_{s}$ was fixed to 4 . Although this first environment has a limited proximity to the real case, it will be very useful to study the extraction performance of CMBS in a very controlled scenario.

\section{Second Environment}

Every recording in the database of this second environment is an AF excerpt of one minute in length that has been extracted from a long term Holter recording. The database consists of 50 real two-lead Holter recordings of AF episodes. Although all recordings were initially sampled at a frequency rate of $128 \mathrm{~Hz}$, each of them was upsampled to a frequency rate of $1024 \mathrm{kHz}$ in order to improve the 
working conditions of the CMBS algorithm. In this second environment the feature, $N_{m}$ cannot be set because the mixing procedure is not controlled. On the contrary, $N_{s}$ can be fixed because it is an input feature of the CBSS algorithm. Attending to the results of the Infomax algorithm, $N_{s}$ was fixed to 4 .

\subsubsection{Performance Indicators}

Given that the CMBS algorithm includes a wavelet stage, the $S I R_{A A}$ index cannot be used to assess the AA extraction performance because the wavelet stage hinders its application. Note that neither the $w_{i j}$ elements of equation 6.9 nor $\mathrm{w}_{\mathrm{AA}}$ of equation 6.16 are known because they are hidden by the wavelet stage. For this reason, neither equation equation 6.11 nor equation 6.17 can be figured out. Consequently, other indicators were taken into account to test the CMBS performance.

In the first environment, four performance indicators were computed from the extracted AA. The first of these indicators is the cross-correlation index in the time domain, $R_{A A t}$, which was already introduced in equation 6.18. An additional indicator is the cross-correlation in the frequency domain, $R_{A A f}$, which evaluates the level of likelihood between the Fourier transforms of the original an the estimated AA. Besides, it is also evaluated whether the main peak frequency, $f_{p 1}$, is in the expected rank from 4 to $8 \mathrm{~Hz}$. Finally, the $S C$ indicator, previously defined in equation 6.20 is used here as a measure of the extraction performance, seeing that the $S C$ is highly correlated with $R_{A A_{t}}$ and with the mean square error (MSE) between the real and the estimated AA. $S C$ values higher than $60 \%$ ensure high values of $R_{A A_{t}}$ and low values of MSE, and denotes a proper PSD of the estimated AA [93].

In the second environment, only the two last indexes were applicable since the original AA is unknown. Since the $R_{A A_{t}}$ and the MSE cannot be computed, the $S C$ was deemed to be a good alternative to assess the AA extraction from real signals. On the other hand, the $f_{p 1}$ can be also computed in this environment and used as a criterium of AA extraction suitability based on the aforementioned expected rank.

\subsection{Performance Analysis of AF Termination Predic- tion}

The performance analysis of $\mathrm{AF}$ termination prediction requires, as the previous study of CBSS and CMBS, the definition of the testing environment, which will be described in subsection 6.4.1. On the other hand, the methodology of MPSE algorithm has been explained in section 5.2, but the spectral features have not been specified yet. In subsection 6.4.2, these spectral features are introduced. Finally, 
the assessment of quantization effects compelled the introduction of specific performance indicators, which will be defined in subsection 6.4.3.

\subsubsection{Testing Environment}

Only one environment and a unique database are used to evaluate the termination prediction. For the present work we have used the database of PhysioNet/Computers in Cardiology Challenge 2004, which consists of 50 two-lead Holter recordings of PAF episodes. Each recording in the database is a one minute segment of PAF that has been extracted from a long term Holter recording. The database is divided into a learning set and a test set. The learning set contains 10 segments of non-terminating AF (group N) and 10 segments of AF that terminates within a second after the end of the record (group T). The test set contains 30 records, approximately half of them belonging to group $\mathrm{N}$, and the rest to group T. The original sampling rate $\left(f_{s}\right)$ of the Holter system was 128 samples per second, but the ECG recordings were interpolated by zero-padding with a interpolation factor of 8 , so that a $f_{s}$ equal to 1024 resulted. This database is used to test the AA termination prediction performance of both MPSE and Poincaré plots, and the quantization effects as well.

\subsubsection{Spectral Features in MPSE}

The first considered spectral feature is the main peak frequency $\left(f_{p 1}\right)$, which is known to be highly relevant in the characterization of AF [68]. The second spectral feature is the main peak magnitude, $A_{1}$. The third and fourth features are the second largest frequency peak $\left(f_{p 2}\right)$ and its related peak magnitude, $A_{2}$. The extraction of these four features can be visualized in Figure 5.2. The fifth feature is the $(S C)$, which was already defined in equation 6.20 .

Other two features related to the width of the spectrum main lobe have been used: the 3-dB width of the peak, $w 3 d B$, and the power in the 3-dB band, $p b 3 d B$. This two last features have been used in [28] to characterize AF. Two derived features, $\Delta f_{p}$ and $\bar{A}_{2}$ are referred to the spectral shape of AA. Similar features are used in [141]. The first derived feature is the normalized distance between $f_{p 1}$ and $f_{p 2}$, which is expressed as:

$$
\Delta f_{p}=\frac{\left(f_{p 1}-f_{p 2}\right)}{f_{p 1}}
$$

The second derived feature is the normalized amplitude of the second largest peak, which is defined as:

$$
\bar{A}_{2}=\frac{A_{2}}{A_{1}}
$$


The deviation of the main and second peak frequencies from their respective mean values are also computed as a dispersion measurement:

$$
\begin{aligned}
& d_{1}=f_{p 1}-\boldsymbol{E}\left(f_{p 1}\right) \\
& d_{2}=f_{p 2}-\boldsymbol{E}\left(f_{p 2}\right)
\end{aligned}
$$

where $\boldsymbol{E}(\cdot)$ represents the mathematical expectation over the periodogram. Finally, the Median Frequency (MF) is obtained as the center of mass of the spectrum:

$$
M F=\frac{\sum_{f=0}^{0.5 f_{s}}\left|F T_{A A}(f)\right| \cdot f}{\sum_{f=0}^{0.5 f_{s}} f}
$$

where $F T_{A A}$ is the Fourier Transform of the AA. This feature was previously used in other works to characterize the ventricular fibrillation [142].

The SampEn of the constructed series from the aforementioned spectral features series is computed in the MPSE algorithm and used to predict the termination of PAF episodes, as previously described in section 5.2. It is important to remark that this prediction is not based on the raw spectral features, but on the SampEn of their series instead, which estimates their mathematical regularity. The performance of MPSE will be quantified in chapter 7 as the number or percentage of correctly classified AF episodes.

\subsubsection{Performance Indicators of Quantization Effects}

Two performance indicators are used to quantify the effects of signal quantization. On the first hand, the cross-correlation between the AA quantized with 16 bits $\left(A A_{16}\right)$ and the AA quantized with $q$ bits $\left(A A_{q}\right)$ was defined as:

$$
R_{q}=\frac{\boldsymbol{E}\left\{A A_{16} \cdot A A_{q}\right\}}{\sqrt{\boldsymbol{E}\left\{A A_{16}^{2}\right\} \boldsymbol{E}\left\{A A_{q}{ }^{2}\right\}}}
$$

$R_{q}$ was obtained as a measure of signal estimation quality since it quantifies the degree of similarity between signals. On the other hand, the Mean Absolute Error (MAE) index for two vectors $x_{1}$ and $x_{2}$, both of length $N$, is computed as:

$$
M A E=\frac{\sum_{i=1}^{N}\left|x_{1}(i)-x_{2}(i)\right|}{N}
$$

As the name suggests, the mean absolute error is a weighted average of the absolute errors. MAE provides a different point of view on the degree of similarity 
between signals. MAE has the advantage of preserving the same units than vectors $x_{1}$ and $x_{2}$ [94]. MAE will be later applied to the extracted features series $f_{p 1}$ and $S C$ and to the SampEn of AA signals as well. 



\section{Chapter 7}

Results

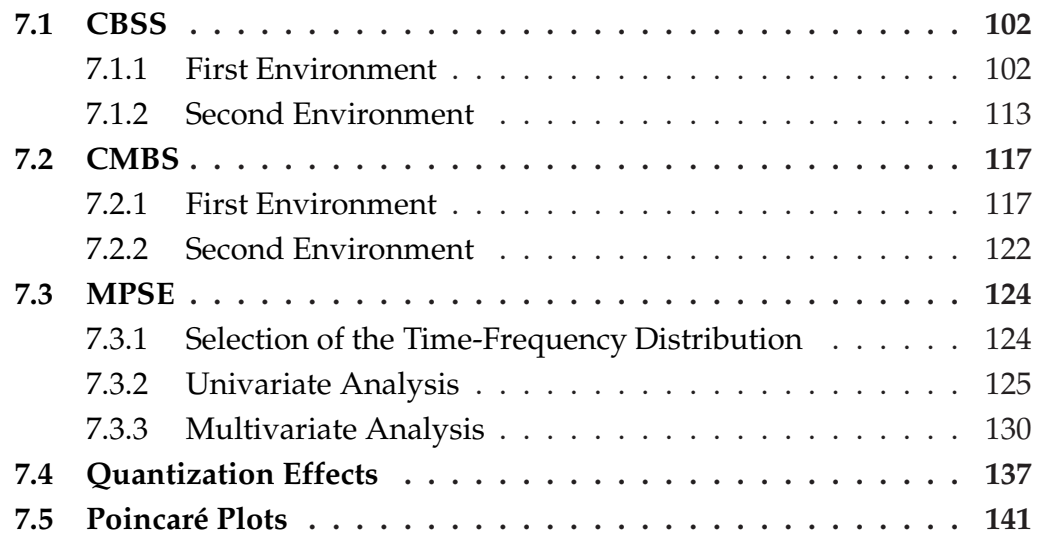

The presentation of results is divided in three main parts, accordingly to the methods arrangement in chapters 4 and 5. In the first place, the AA extraction performance of the analyzed CBSS algorithms is presented in section 7.1. In the second place, the performance of the new algorithm for AA extraction, CMBS, is presented in section 7.2. In the third place, the results of PAF termination prediction achieved by MPSE are presented in section 7.3. The results reached by the visual method based on Poincaré plots are given in section 7.5. Finally, the quantization effects are detailed in section 7.4. 


\subsection{CBSS}

This section is subdivided in two subsections. In subsection 7.1.1 the results of the first environment described in subsection 6.2.1 are presented. Similarly, the results of the second environment, also described in subsection 6.2.1, are exposed in subsection 7.1.2. Remember that the signals mixture of the first environment is a very controlled process and the signals mixture of the second environment is a more realistic one. In addition to the BSS algorithms results, the outcomes of the FastICA algorithm are also included in every figure and table in order to make a comparison between the convolutive and the instantaneous model.

\subsubsection{First Environment}

\section{Infomax}

The mean $R_{A A_{t}}$ - the correlation in time domain between the original and estimated AA - obtained by Infomax is depicted in Figure 7.1 for each $N_{m}$ and $N_{s}$ considered in the analysis. $N_{m}$ and $N_{s}$ were previously introduced in chapter 6 as the filter length of the mixing and separation matrices, respectively. At the same time, the mean $R_{A A_{t}}$ values are tabulated in table 7.1 with three significant digits. With regard to $N_{m}$, a clear trend can be deduced from Figure 7.1: as $N_{m}$ increases the $R_{A A_{t}}$ decreases, what denotes a worsening performance of the algorithm when the mixing process progressively separates from the instantaneous model. With reference to $N_{s}$, the higher values of $R_{A A_{t}}$ are reached for $N_{s}$ equal to one or two, depending on which value of $N_{s}$ is considered. This implies that, in terms of $R_{A A_{t}}$, the Infomax performance is benefitted when a weak convolutive mixing model, near the instantaneous mixing model, is contemplated.

Figure 7.2 shows the mean $S I R_{A A}$ for the Infomax algorithm. Keep in mind that $S I R_{A A}$ quantifies the improvement of Signal to Interference Ratio of AA accomplished by a extraction algorithm. These values of $S I R_{A A}$ are tabulated in table 7.2 with one significant decimal. With reference to $N_{m}$, a similar trend to $R_{A A_{t}}$ is reported, that is, the higher $N_{m}$ the lower $S I R_{A A}$. On the contrary, the maximum values of $S I R_{A A}$ are not reached for $N_{s}$ equal to one but for $N_{s}$ between 4 and 32, depending on the considered $N_{m}$. Since the mixed signals are originally extracted from real signals, this fact could hide a convolutive mixture of real sources so that the AA extraction process is enhanced when a convolutive unmixing process is carried out.

In comparison with FastICA, the mean $R_{A A_{t}}$ values for Infomax are slightly lower than those for FastICA and nearer to one when the optimal values of $N_{m}$ and $N_{s}$ are considered. Similarly, the mean $S I R_{A A}$ values for Infomax are a few decibels lower than the mean $S I R_{A A}$ for FastICA. The best mean $S I R_{A A}$ values are around $30 d B$ for Infomax, and around $35 d B$ for FastICA. This means that 
the AA extraction performance of Infomax is quite good but does not exceed the extraction performance of FastICA. This is interpreted as an adaptation necessity of the the Infomax algorithm to the particular problem of AA extraction.

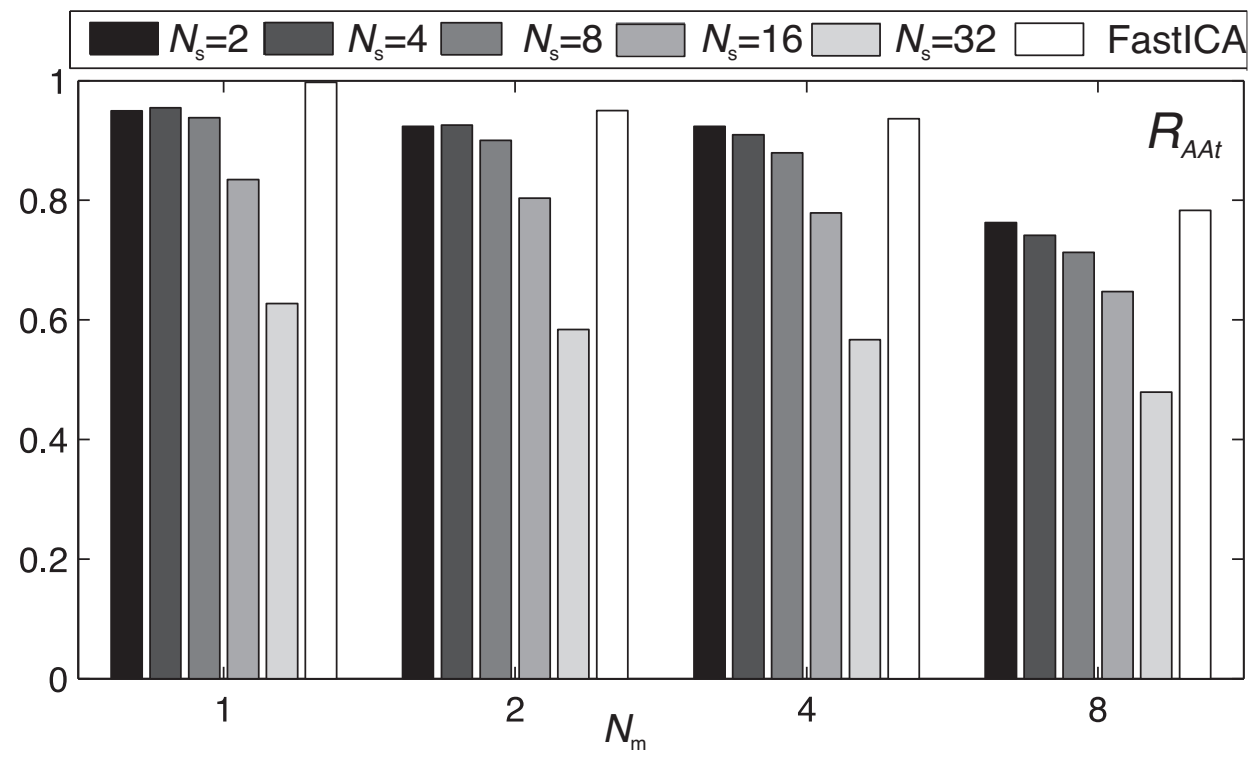

Figure 7.1. Mean $R_{A A_{t}}$ for the Infomax algorithm in the first environment. The length of the mixing matrix filters $N_{m}$ is varied from 1 to 8 and the length of the separation matrix filters $N_{s}$ from 2 to 32 . The values for the FastlCA algorithm are also included for comparison.

\begin{tabular}{l|c|c|c|c|}
\cline { 2 - 5 }$N_{s}$ & \multicolumn{4}{|c|}{$N_{m}$} \\
\hline 2 & $0.949 \pm 0.127$ & $0.924 \pm 0.141$ & $0.924 \pm 0.119$ & $0.763 \pm 0.247$ \\
4 & $0.955 \pm 0.091$ & $0.926 \pm 0.111$ & $0.910 \pm 0.119$ & $0.741 \pm 0.245$ \\
8 & $0.938 \pm 0.079$ & $0.900 \pm 0.115$ & $0.876 \pm 0.120$ & $0.713 \pm 0.238$ \\
16 & $0.835 \pm 0.134$ & $0.804 \pm 0.141$ & $0.779 \pm 0.150$ & $0.647 \pm 0.222$ \\
32 & $0.627 \pm 0.162$ & $0.584 \pm 0.193$ & $0.567 \pm 0.180$ & $0.479 \pm 0.203$ \\
\hline FastICA & $0.997 \pm 0.014$ & $0.930 \pm 0.113$ & $0.937 \pm 0.125$ & $0.783 \pm 0.247$ \\
\hline
\end{tabular}

Table 7.1. Infomax mean $R_{A A_{t}}$ of Figure 7.1 expressed with three significant digits.

\section{MBLMS}

Figure 7.3 shows the mean $R_{A A_{t}}$ of MBLS for $N_{m}$ equal to 1, 2, 4 and 8, and $N_{s}$ equal to 2,4 8,16 and 32. These mean values are tabulated in table 7.3 with three significant digits. The mean $R_{A A_{t}}$ decreases as $N_{m}$ increases and reaches a maximum for $N_{m}=1$ and $N_{s}=2$. This maximum of $R_{A A_{t}}$ is equal to $0.232 \pm$ 0.194, which denotes the low ability of MBLS to AA extraction. 


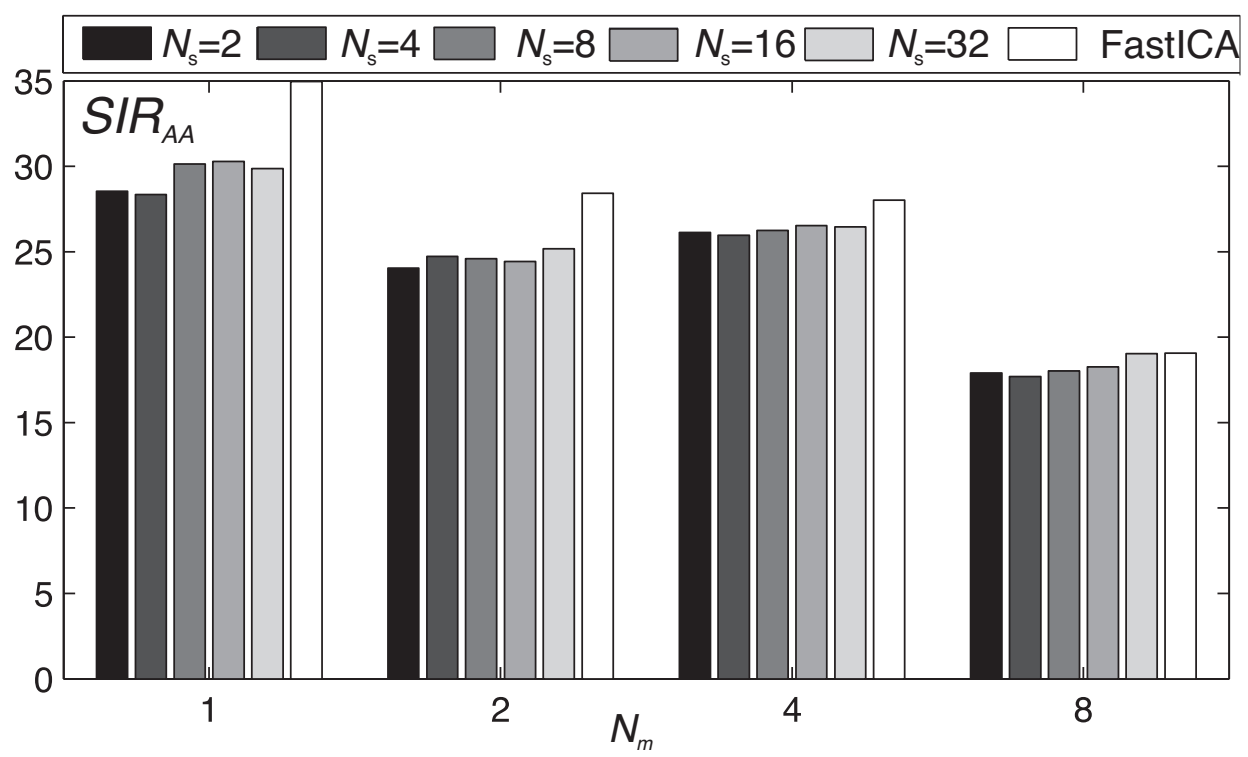

Figure 7.2. Mean $S I R_{A A}(d B)$ for the Infomax algorithm in the first environment. The length of the mixing matrix filters $N_{m}$ is varied from 1 to 8 and the length of the separation matrix filters $N_{s}$ from 2 to 32. The values for the FastlCA algorithm are also included for comparison.

\begin{tabular}{l|c|c|c|c|}
\cline { 2 - 5 }$N_{s}$ & \multicolumn{4}{|c|}{$N_{m}$} \\
\hline 2 & $28.5 \pm 14.2$ & $24.0 \pm 9.6$ & $26.1 \pm 6.7$ & $17.9 \pm 8.6$ \\
4 & $28.4 \pm 13.9$ & $24.7 \pm 10.0$ & $26.0 \pm 6.8$ & $17.7 \pm 8.7$ \\
8 & $30.1 \pm 15.5$ & $24.6 \pm 10.2$ & $26.3 \pm 6.9$ & $18.0 \pm 8.7$ \\
16 & $30.3 \pm 16.2$ & $24.4 \pm 10.4$ & $26.5 \pm 7.4$ & $18.3 \pm 8.7$ \\
32 & $29.9 \pm 15.1$ & $25.2 \pm 10.9$ & $26.5 \pm 8.0$ & $19.0 \pm 9.5$ \\
\hline FastICA & $35.0 \pm 9.9$ & $28.4 \pm 8.2$ & $28.0 \pm 7.3$ & $19.1 \pm 9.0$ \\
\hline
\end{tabular}

Table 7.2. Infomax mean $S I R_{A A}(d B)$ of Figure 7.2 expressed with one significant decimal. 
The mean $S I R_{A A}$ for MBLMS are depicted in Figure 7.4 and tabulated in table 7.4 with one significant decimal. A low performance of MBLSM is also observed in terms of $S I R_{A A}$, since this index is always lower than $5 \mathrm{~dB}$. More specifically, the maximum mean $S I R_{A A}$ is reached for $N_{m}$ equal to 2 and $N_{s}$ equal to 16 , and equals $2.7 \pm 3.2 \mathrm{~dB}$. In spite of these $S I R_{A A}$ low values, it can be perceived a slight performance improvement of MBLMS when a convolutive model is considered in the mixing and separation processes instead of the instantaneous model.

The performance comparison of MBLMS and FastICA algorithms lead to the same deduction either $R_{A A_{t}}$ or $S I R_{A A}$ are regarded. SIR $R_{A A}$ values obtained by FastICA algorithm always exceed those obtained by MBLMS algorithm. SIR $R_{A A}$ FastICA values are near to $40 \mathrm{~dB}$. Meanwhile, $S I R_{A A}$ MBLMS values are near to $0 \mathrm{~dB}$. With reference to $R_{A A_{t}}$, the mean values for FastICA are always near one. On the contrary, $R_{A A_{t}}$ for MBLMS always approximates zero. That is, the MBLMS algorithm is unable to reach a practical source signal separation from AF ECG.

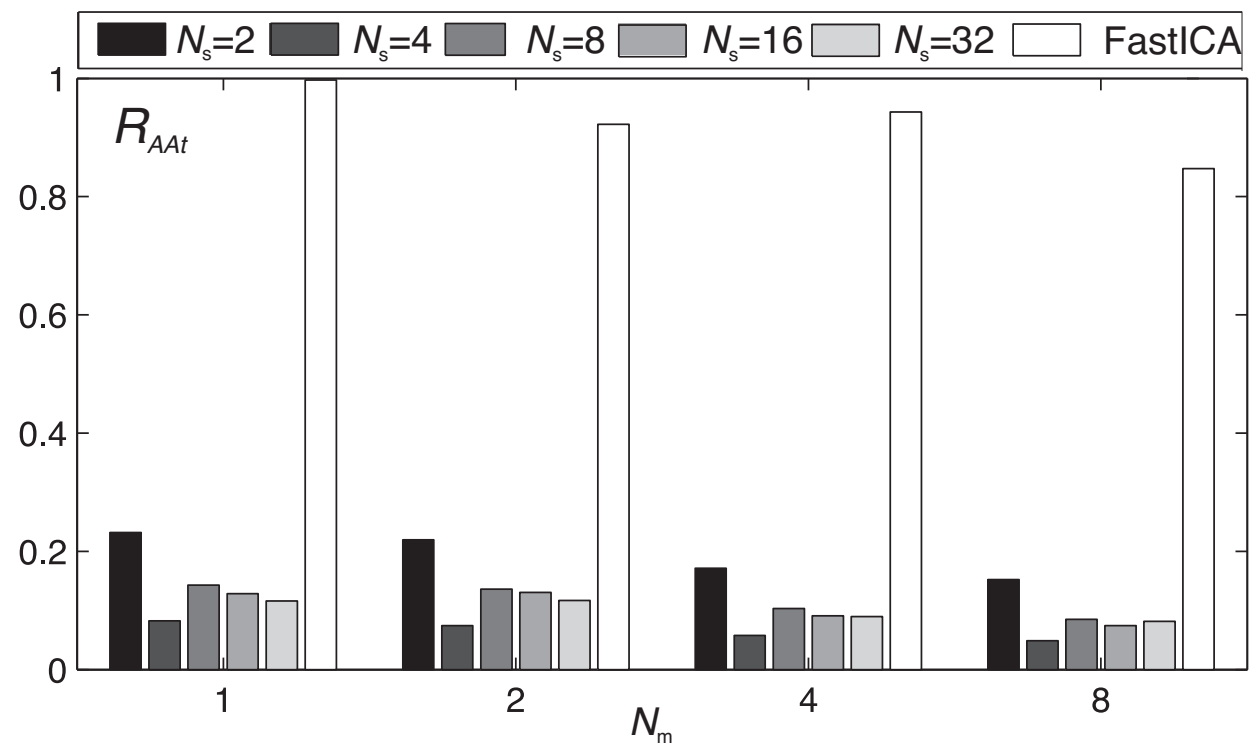

Figure 7.3. Mean $R_{A A_{t}}$ for the MBLMS algorithm in the first environment. The length of the mixing matrix filters $N_{m}$ is varied from 1 to 8 and the length of the separation matrix filters $N_{s}$ from 2 to 32 . The values for the FastlCA algorithm are also included for comparison.

\section{TDD}

The mean $R_{A A_{t}}$ reached by TDD is depicted in Figure 7.5 and tabulated with three significant digits in table 7.5 for the values of $N_{m}$ and $N_{s}$ considered in the analysis. The mean $R_{A A_{t}}$ values obtained by TDD are much higher than those obtained by MBLMS for every $N_{m}$ and $N_{s}$. The mean $R_{A A_{t}}$ for TDD is comparable 


\begin{tabular}{l|c|c|c|c|}
\cline { 2 - 5 }$N_{s}$ & \multicolumn{4}{|c|}{$N_{m}$} \\
\hline 2 & $0.232 \pm 0.194$ & $0.220 \pm 0.143$ & $0.172 \pm 0.118$ & $0.152 \pm 0.103$ \\
4 & $0.082 \pm 0.076$ & $0.074 \pm 0.048$ & $0.058 \pm 0.032$ & $0.049 \pm 0.019$ \\
8 & $0.143 \pm 0.126$ & $0.136 \pm 0.096$ & $0.103 \pm 0.088$ & $0.085 \pm 0.075$ \\
16 & $0.129 \pm 0.109$ & $0.131 \pm 0.081$ & $0.091 \pm 0.068$ & $0.074 \pm 0.046$ \\
32 & $0.116 \pm 0.080$ & $0.116 \pm 0.077$ & $0.089 \pm 0.070$ & $0.081 \pm 0.063$ \\
\hline FastICA & $0.997 \pm 0.002$ & $0.923 \pm 0.183$ & $0.943 \pm 0.115$ & $0.847 \pm 0.182$ \\
\hline
\end{tabular}

Table 7.3. MBLMS mean $R_{A A_{t}}$ of Figure 7.3 expressed with trhee significant digits.

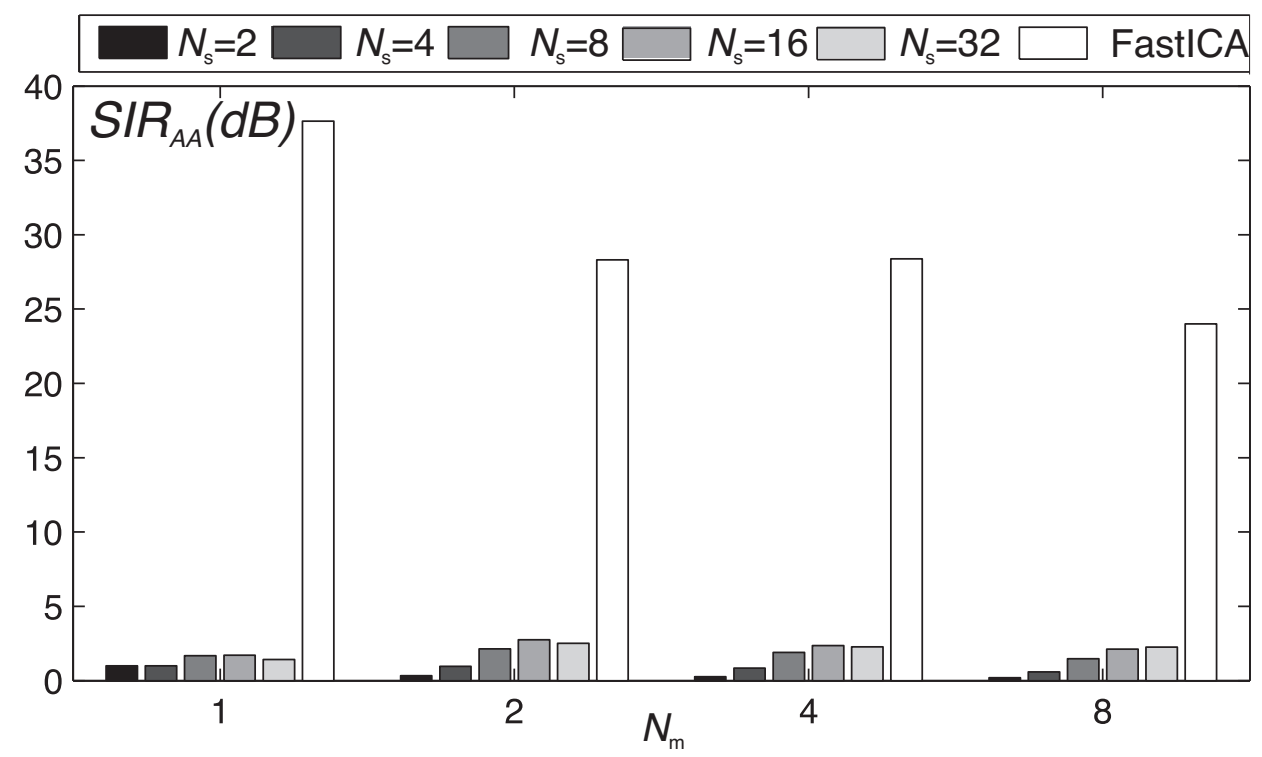

Figure 7.4. Mean $S I R_{A A}(d B)$ for the MBLMS algorithm in the first environment. The length of the mixing matrix filters $N_{m}$ is varied from 1 to 8 and the length of the separation matrix filters $N_{s}$ from 2 to 32. The values for the FastICA algorithm are also included for comparison.

\begin{tabular}{l|c|c|c|c|}
\cline { 2 - 5 }$N_{s}$ & \multicolumn{4}{|c|}{$N_{m}$} \\
\hline 2 & $1.0 \pm 2.3$ & $0.3 \pm 0.3$ & $0.3 \pm 0.3$ & $0.2 \pm 0.2$ \\
4 & $1.0 \pm 2.2$ & $1.0 \pm 0.9$ & $0.8 \pm 1.0$ & $0.6 \pm 0.7$ \\
8 & $1.7 \pm 4.5$ & $2.1 \pm 2.6$ & $1.9 \pm 2.5$ & $1.5 \pm 2.0$ \\
16 & $1.7 \pm 5.8$ & $2.7 \pm 3.2$ & $2.4 \pm 2.4$ & $2.1 \pm 3.0$ \\
32 & $1.4 \pm 6.6$ & $2.5 \pm 2.9$ & $2.3 \pm 2.6$ & $2.3 \pm 3.1$ \\
\hline FastICA & $37.6 \pm 17.0$ & $28.3 \pm 10.4$ & $28.4 \pm 7.5$ & $24.0 \pm 8.7$ \\
\hline
\end{tabular}

Table 7.4. MBLMS mean $S I R_{A A}(d B)$ of Figure 7.4 expressed with one significant decimal. 
to the mean $R_{A A_{t}}$ for Infomax since similar maximum values are achieved. Similarly to Infomax, the mean $R_{A A_{t}}$ decreases when $N_{m}$ increases. This indicates, in terms of the $R_{A A_{t}}$ index, an improvement of AA extraction for TDD when a weak convolutive model near to the instantaneous model is accomplished in the mixing process.

Figure 7.6 shows the mean $S I R_{A A}$ for the TDD algorithm. The same values are tabulated in table 7.6 with one significant decimal. As for $R_{A A_{t}}$, the mean values of $S I R_{A A}$ are comparable to those of Infomax, and reach values near 30 $\mathrm{dB}$. With reference to the mixing process, the higher $N_{m}$ the lower the $S I R_{A A}$ is. That is, the algorithm performance is helped by considering a instantaneous linear mixing model. Nonetheless, the effect on $S I R_{A A}$ of $N_{s}$ does not indicate the same. On the contrary the higher values of $S I R_{A A}$ are not reached for $N_{m}$ equal to one but for $N_{m}$ between 8 and 32. This suggests, as in the Infomax case, that the original mixed sources might be generated by a convolutive mixing so that a better AA extraction is accomplished when a convolutive unmixing process is executed.

From Figures 7.5 and 7.6 it can be concluded that both $R_{A A_{t}}$ values and $S I R_{A A}$ values are of the same order than those for FastICA. Specifically, the maximum mean $R_{A A_{t}}$ for TDD equals $0.960 \pm 0.091\left(N_{m}=1, N_{s}=2\right)$ and the maximum mean $R_{A A_{t}}$ for FastICA is equal to $0.997 \pm 0.002\left(N_{m}=1\right)$. The maximum mean $S I R_{A A}$ for TDD is equal to $32.8 \pm 15.9 \mathrm{~dB}\left(N_{m}=1, N_{s}=16\right)$ and the maximum mean $S I R_{A A}$ for FastICA is equal to $37.0 \pm 8.3 \mathrm{~dB}\left(N_{m}=1\right)$. As expected, the best values for FastICA are reached when $N_{m}$ is equal to one, that is, the instantaneous mixing model is considered. These results show that TDD is a good CBSS algorithm for the AA extraction but, as Infomax, needs to be adapted to this particular problem in order to reach the results obtained by algorithms that follow the instantaneous mixing model as FastICA.

\begin{tabular}{l|c|c|c|c|}
\cline { 2 - 5 }$N_{s}$ & \multicolumn{4}{|c|}{$N_{m}$} \\
\hline 2 & $0.960 \pm 0.091$ & $0.915 \pm 0.176$ & $0.900 \pm 0.144$ & $0.809 \pm 0.194$ \\
4 & $0.958 \pm 0.122$ & $0.915 \pm 0.195$ & $0.893 \pm 0.178$ & $0.813 \pm 0.233$ \\
8 & $0.922 \pm 0.200$ & $0.897 \pm 0.217$ & $0.864 \pm 0.215$ & $0.789 \pm 0.273$ \\
16 & $0.902 \pm 0.212$ & $0.839 \pm 0.255$ & $0.821 \pm 0.245$ & $0.742 \pm 0.291$ \\
32 & $0.827 \pm 0.264$ & $0.784 \pm 0.292$ & $0.737 \pm 0.300$ & $0.705 \pm 0.294$ \\
\hline FastICA & $0.997 \pm 0.002$ & $0.950 \pm 0.147$ & $0.929 \pm 0.134$ & $0.857 \pm 0.204$ \\
\hline
\end{tabular}

Table 7.5. TDD mean $R_{A A_{t}}$ of Figure 7.5 expressed with trhee significant digits. 


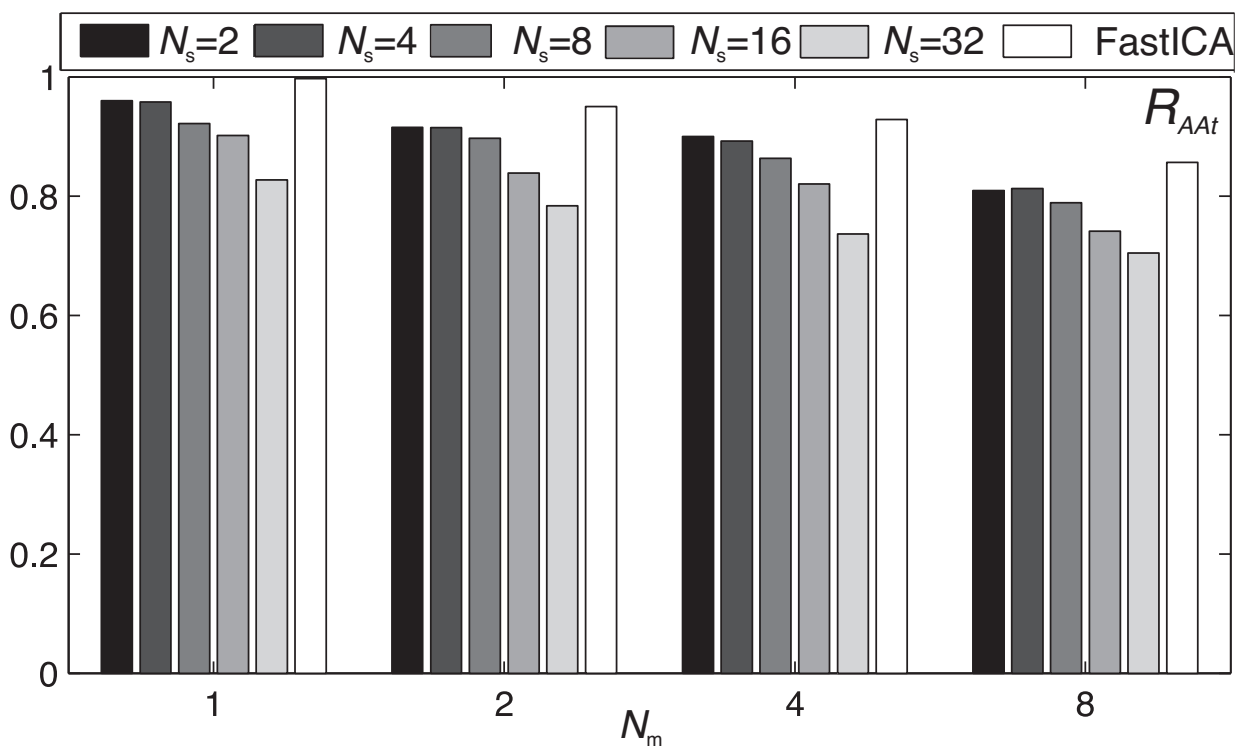

Figure 7.5. Mean $R_{A A_{t}}$ for the TDD algorithm in the first environment. The length of the mixing matrix filters $N_{m}$ is varied from 1 to 8 and the length of the separation matrix filters $N_{s}$ from 2 to 32 . The values for the FastICA algorithm are also included for comparison.

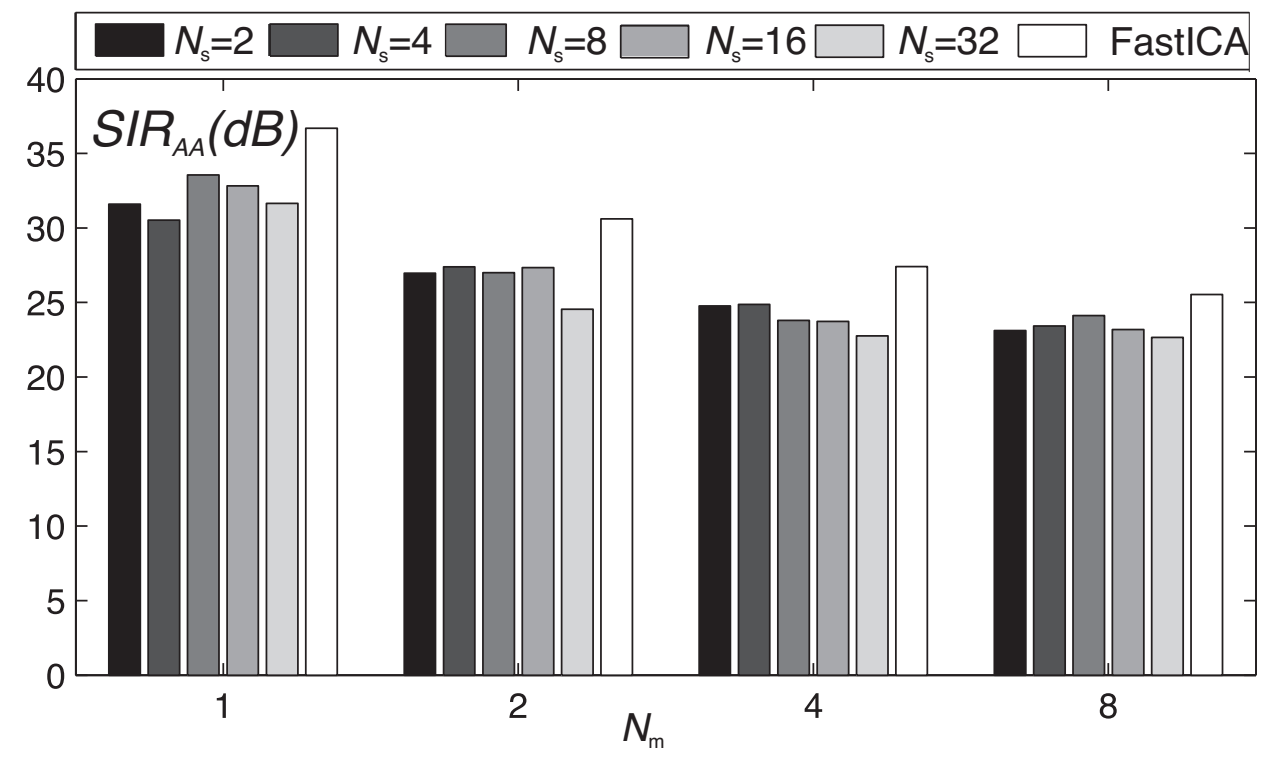

Figure 7.6. Mean $S I R_{A A}(d B)$ for the TDD algorithm in the first environment. The length of the mixing matrix filters $N_{m}$ is varied from 1 to 8 and the length of the separation matrix filters $N_{s}$ from 2 to 32 . The values for the FastICA algorithm are also included for comparison. 


\begin{tabular}{l|c|c|c|c|}
\cline { 2 - 5 }$N_{s}$ & \multicolumn{4}{|c|}{$N_{m}$} \\
\hline 2 & $31.6 \pm 12.6$ & $27.0 \pm 9.9$ & $24.8 \pm 9.0$ & $23.1 \pm 8.1$ \\
4 & $30.5 \pm 10.4$ & $27.4 \pm 8.0$ & $24.9 \pm 7.7$ & $23.4 \pm 8.2$ \\
8 & $33.6 \pm 19.4$ & $27.0 \pm 10.9$ & $23.8 \pm 9.3$ & $24.1 \pm 8.9$ \\
16 & $32.8 \pm 15.9$ & $27.3 \pm 10.7$ & $23.7 \pm 9.6$ & $23.2 \pm 9.1$ \\
32 & $31.7 \pm 14.0$ & $24.6 \pm 10.4$ & $22.8 \pm 9.4$ & $22.7 \pm 9.1$ \\
\hline FastICA & $37.0 \pm 8.3$ & $31.0 \pm 7.2$ & $27.4 \pm 7.5$ & $25.5 \pm 6.7$ \\
\hline
\end{tabular}

Table 7.6. TDD mean $S I R_{A A}(d B)$ of Figure 7.6 expressed with one significant decimal.

\section{CoBliss}

The mean $R_{A A_{t}}$ attained by the CoBliSS algorithm is depicted for $N_{m}=1,2,4,8$ and $N_{s}=2,4,8,16,32$ in Figure 7.7. These values are also tabulated in table 7.7 with three significant digits. Note that, on the contrary to the others tested CBSS algorithms, CoBliSS allows to set $N_{s}$ equal to one. The best $R_{A A_{t}}$ outcome is reached for $N_{m}=1$ and $N_{s}=1$ as well. In this case, the mean $R_{A A_{t}}$ equals 0.893 and is comparable to the maximum mean values of $R_{A A_{t}}$ reached by Infomax and TDD. In contrast, the $R_{A A_{t}}$ of CoBliSS decreases more rapidly when $N_{s}$ increases That is, the performance of CoBliSS, in terms of $R_{A A_{t}}$, is more damaged than the performance of Infomax and TDD when the unmixing process becomes separated by considering the instantaneous model. Nonetheless, an exception is found for $N_{m}=8$, where the maximum mean $R_{A A_{t}}$ is reached for $N_{s}=2$. This means that, for $N_{m}$ equal to 8 , the best estimated separation matrix is reached when the length of its filters is equal to 2 , what reveals a certain convolutive behavior of the sources mixture.

The mean $S I R_{A A}$ for CoBliSS is shown in Figure 7.8 and tabulated in table 7.8 for the aforementioned values of $N_{m}$ and $N_{s}$. The mean $S I R_{A A}$ for CoBliSS is higher than the mean $S I R_{A A}$ for MBLMS but lower than for Infomax an TDD. Meanwhile the maximum $S I R_{A A}$ values of Infomax and TDD are around $30 \mathrm{~dB}$, those of CoBliSS are around $20 \mathrm{~dB}$, that is, $10 \mathrm{~dB}$ lower. The CoBliSS performance occupies an intermediate position between MBLMS algorithm and Infomax and TDD algorithms. This means that CoBlisS is less reliable for the AA extraction from AF ECGs and an adaptation to this particular problem is more unlikely.

Finally, the comparison with FastICA leads to the same deduction either $R_{A A_{t}}$ or $S I R_{A A}$ is regarded. The reached quality of the extracted AA is always better for FastICA whatever the values of $N_{m}$ and $N_{s}$ are. 


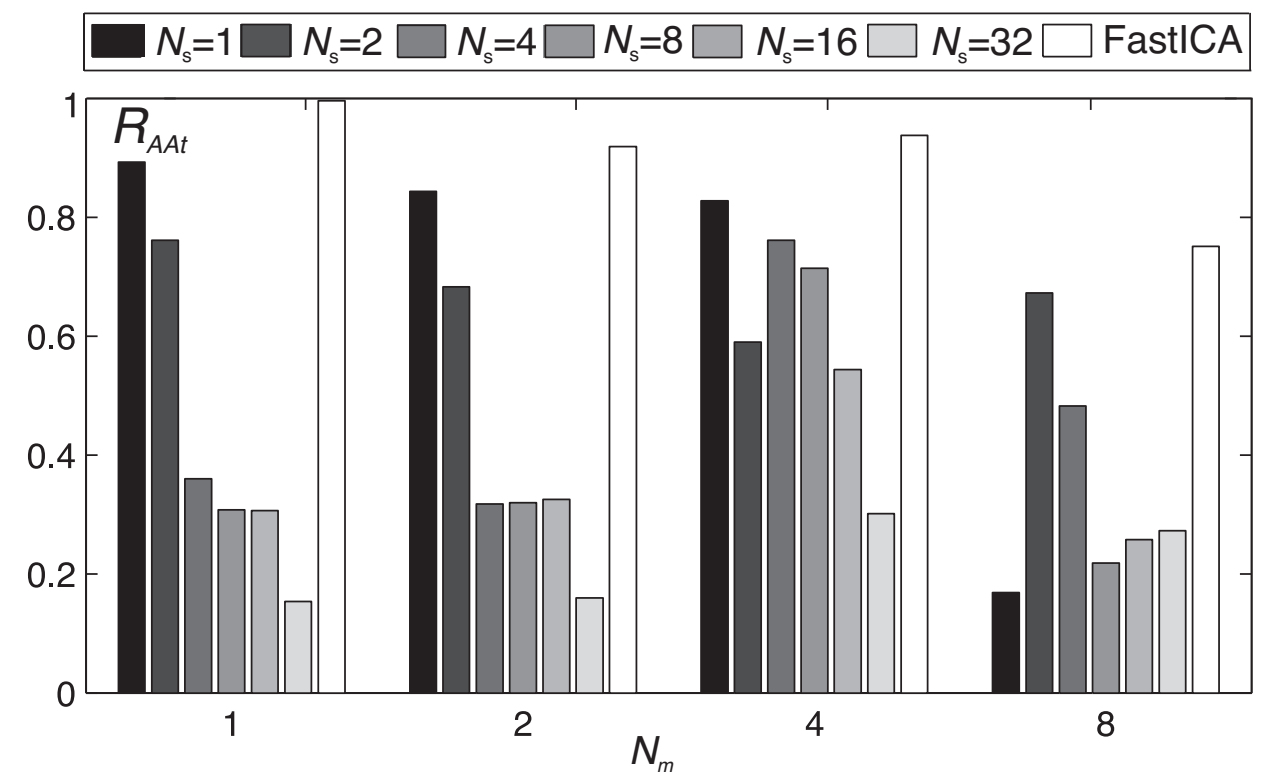

Figure 7.7. Mean $R_{A A_{t}}$ for the CoBliSS algorithm in the first environment. The length of the mixing matrix filters $N_{m}$ is varied from 1 to 8 and the length of the separation matrix filters $N_{s}$ from 1 to 32 . The values for the FastlCA algorithm are also included for comparison.

\begin{tabular}{l|c|c|c|c|}
\cline { 2 - 5 }$N_{s}$ & \multicolumn{4}{|c|}{$N_{m}$} \\
\hline 1 & $0.893 \pm 0.133$ & $0.844 \pm 0.197$ & $0.828 \pm 0.149$ & $0.169 \pm 0.097$ \\
2 & $0.762 \pm 0.104$ & $0.683 \pm 0.188$ & $0.590 \pm 0.266$ & $0.673 \pm 0.224$ \\
4 & $0.360 \pm 0.152$ & $0.318 \pm 0.160$ & $0.761 \pm 0.234$ & $0.482 \pm 0.194$ \\
8 & $0.308 \pm 0.160$ & $0.320 \pm 0.162$ & $0.714 \pm 0.144$ & $0.218 \pm 0.139$ \\
16 & $0.307 \pm 0.179$ & $0.326 \pm 0.175$ & $0.544 \pm 0.144$ & $0.258 \pm 0.147$ \\
32 & $0.154 \pm 0.130$ & $0.160 \pm 0.121$ & $0.301 \pm 0.149$ & $0.273 \pm 0.143$ \\
\hline FastICA & $0.996 \pm 0.020$ & $0.919 \pm 0.190$ & $0.938 \pm 0.126$ & $0.751 \pm 0.261$ \\
\hline
\end{tabular}

Table 7.7. CoBliSS mean $R_{A A_{t}}$ of Figure 7.7 expressed with trhee significant digits. 


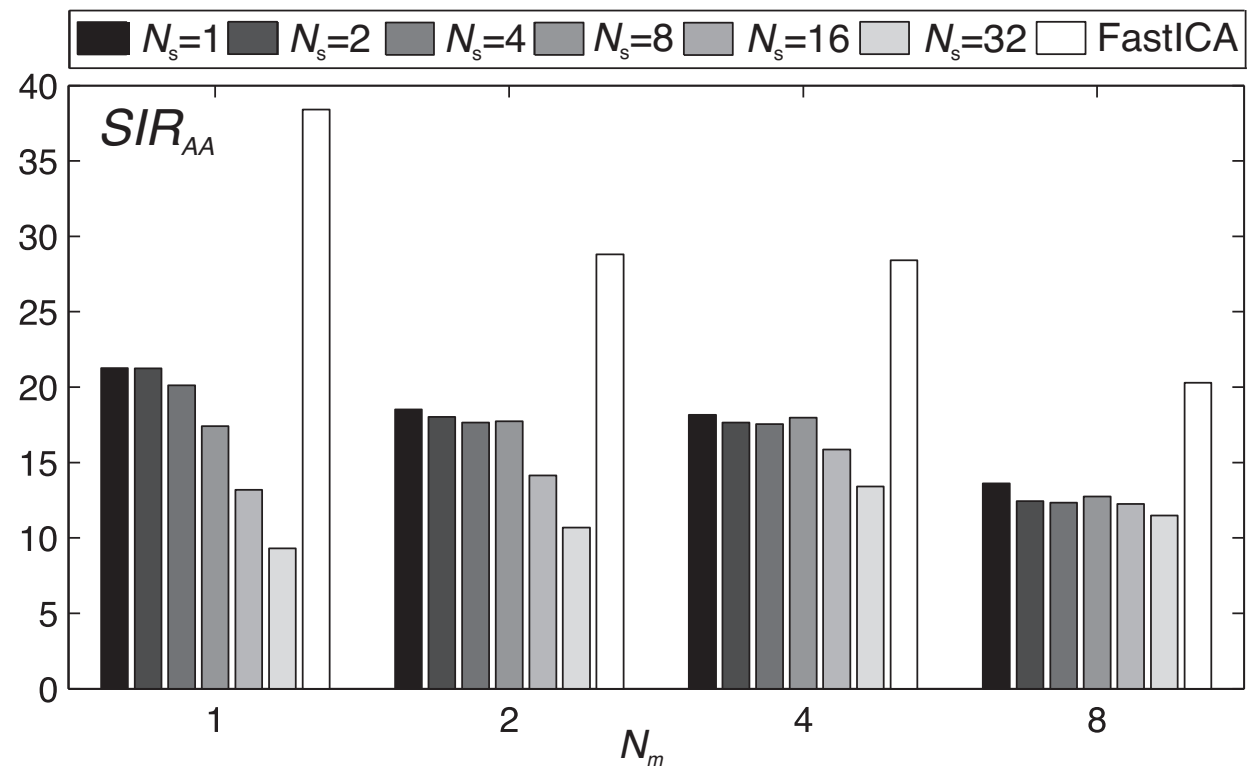

Figure 7.8. Mean $S I R_{A A}(d B)$ for the CoBliSS algorithm in the first environment. The length of the mixing matrix filters $N_{m}$ is varied from 1 to 8 and the length of the separation matrix filters $N_{s}$ from 1 to 32. The values for the FastlCA algorithm are also included for comparison.

\begin{tabular}{l|c|c|c|c|}
\cline { 2 - 5 }$N_{s}$ & \multicolumn{4}{|c|}{$N_{m}$} \\
\hline 1 & $21.3 \pm 10.6$ & $18.5 \pm 8.2$ & $18.2 \pm 5.9$ & $13.6 \pm 6.0$ \\
2 & $21.3 \pm 10.6$ & $18.5 \pm 8.2$ & $18.2 \pm 5.9$ & $13.6 \pm 5.6$ \\
4 & $21.2 \pm 9.6$ & $18.0 \pm 9.2$ & $17.7 \pm 5.9$ & $12.4 \pm 6.4$ \\
8 & $20.1 \pm 7.9$ & $17.7 \pm 7.4$ & $17.6 \pm 5.5$ & $12.3 \pm 6.7$ \\
16 & $17.4 \pm 8.4$ & $17.7 \pm 6.4$ & $18.0 \pm 5.2$ & $12.8 \pm 7.1$ \\
32 & $13.2 \pm 7.3$ & $14.1 \pm 6.8$ & $15.9 \pm 5.9$ & $12.3 \pm 6.4$ \\
\hline FastICA & $38.4 \pm 13.9$ & $28.8 \pm 10.7$ & $28.4 \pm 7.6$ & $20.3 \pm 10.1$ \\
\hline
\end{tabular}

Table 7.8. CoBliSS mean $S I R_{A A}(d B)$ of Figure 7.8 expressed with one significant decimal. 


\section{Joint Description}

Now a comparison of the CBSS algorithms performance is carried out. In order to facilitate the analysis, the output data were reorganized. Figure 7.9 shows the mean $S I R_{A A}$ and the mean $R_{A A_{t}}$ of the tested CBSS algorithms in the first environment as a function of the mixing matrix filters length $\left(N_{m}\right)$ regardless of the separation matrix filters length $\left(N_{s}\right)$. Conversely, Figure 7.10 shows the mean $S I R_{A A}$ and the mean $R_{A A_{t}}$ of the tested CBSS algorithms in the first environment as a function of $N_{s}$ regardless of $N_{m}$. These values are also tabulated in tables 7.9, $7.10,7.11$, and 7.12, respectively.

It can be appreciated in Figure 7.9.a that FastICA $S I R_{A A}$ mean values are higher than MBLMS SIR $A A$ mean values for any value of $N_{m}$. More specifically, maximum FastICA $S I R_{A A}$ mean values are around $40 \mathrm{~dB}$ whereas MBLMS $S I R_{A A}$ mean values are always lower than $5 \mathrm{~dB}$. In other words, the application of MBLMS to mixtures of AA an VA does not yield any source signal separation. On the contrary, the values obtained by TDD are much better than values obtained by MBLMS. In fact, $S I R_{A A}$ mean values of FastICA are only around $10 \mathrm{~dB}$ higher than TDD $S I R_{A A}$ mean values for instantaneous mixtures $\left(N_{m}=1\right)$. Furthermore, both values decrease and tend to be equal when $N_{m}$ increases. Infomax presents a similar behavior to TDD, that is, the $S I R_{A A}$ mean values are slightly lower than the respective FastICA values for instantaneous mixtures and much more similar when $N_{m}$ increases. Interestingly, the mean $S I R_{A A}$ of Infomax for $N_{m}=4(26.3 \pm 72 \mathrm{~dB})$ is is higher than for $N_{m}=2(24.6 \pm 10.2 \mathrm{~dB})$, which can be interpreted as a convolutive component of the sources mixture. Finally, CoBliSS $S I R_{A A}$ mean values are around two decades lower than FastICA SIR $R_{A A}$ mean values, that is, the performance of FastICA in the extraction of the AA is much better than the performance obtained by MBLMS but does not reach the performance obtained by TDD and Infomax in any case. The maximum mean $S I R_{A A}$ of CoBliSS occurs for $N_{m}=4(16.9 \pm 5.7 \mathrm{~dB})$. As for Infomax, this can be viewed as a convolutive mixture of sources.

In Figure $7.9 \mathrm{~b}$ similar trends of the AA extraction quality can be observed in terms of the $R_{A A_{t}}$ feature. TDD and Infomax are the CBSS algorithms which $R_{A A_{t}}$ mean values are nearer to one, and they tend to the FastICA $R_{A A_{t}}$ values when $N_{m}$ increases. The curve of CoBliSS stands out because the maximum $R_{A A_{t}}$ is reached for $N_{m}=4(0.626 \pm 0.181)$, what reaffirms the hypothesis of convolutive mixtures.

Figure 7.10 and tables 7.11 and 7.12 illustrate the influence of the length of the separation matrix filters $\left(N_{s}\right)$ in the quality of the extraction. $N_{s}$ varies in this figure and these tables from 2 to 32. The case $N_{s}=1$ is not included since it is only available for the CoBliSS algorithm. On the other hand, the mean values of FastICA are just included as a reference constant value, although FastICA does not match the convolutive model and, therefore, the filters length feature cannot be chosen. The mean $R_{A A_{t}}$ and mean $S I R_{A A}$ of TDD and Infomax are very close to those of FastICA. An interesting trend can be observed in Figure 7.10.a: the 
increasing mean Infomax $S I R_{A A}$ when $N_{s}$ increases, with a maximum for $N_{s}=$ $32(25.1 \pm 10.8 \mathrm{~dB})$. Another important detail is the maximum mean $S I R_{A A}$ for $\operatorname{TDD}(27.1 \pm 12.1 \mathrm{~dB})$, that occurs when $N_{s}$ is equal to 8 . Furthermore, although the $S I R_{A A}$ mean values for MBLMS are very low, they also increase accordingly to the values of $N_{s}$. Figure 7.11.b shows that the mean $R_{A A_{t}}$ of each CBSS algorithm approaches that of FastICA for $N_{s}=1$ but, conversely to $N_{m}$, these mean values decrease when $N_{s}$ increases.

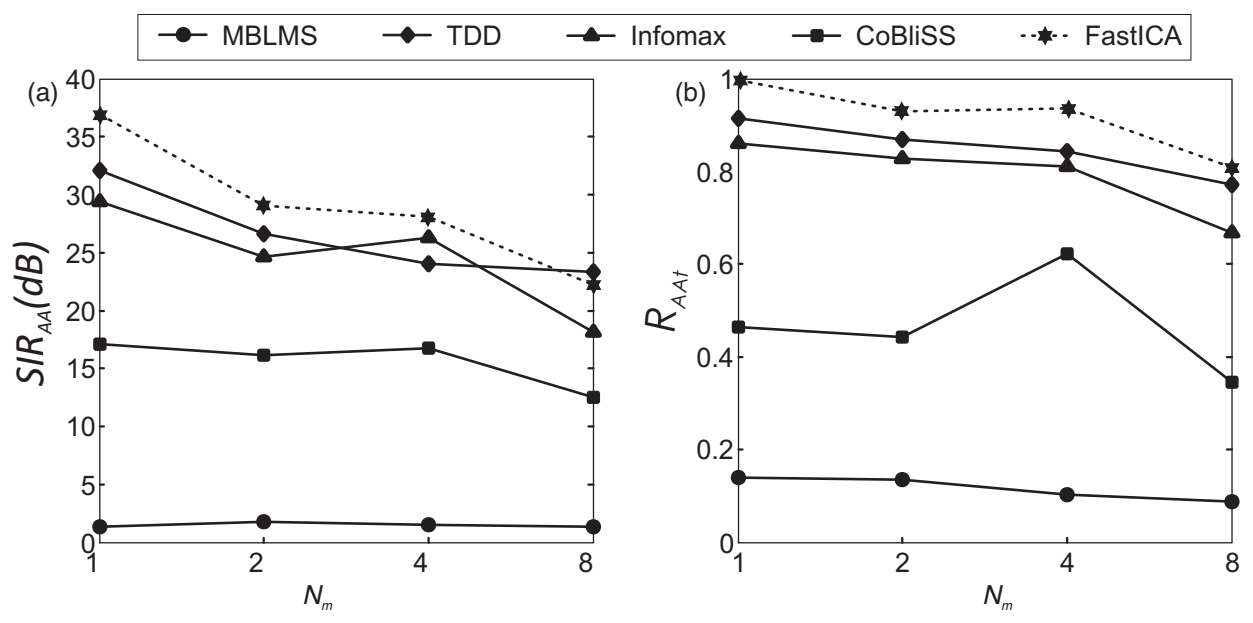

Figure 7.9. CBSS algorithms (a) $S I R_{A A}$ and (b) $R_{A A_{t}}$ mean values in the first environment as a function of the mixing matrix filters length $\left(N_{m}\right)$ regardless of the separation matrix filters length $\left(N_{s}\right)$.

\begin{tabular}{l|l|l|l|l|l|}
$N_{s}$ & MBLMS & TDD & Infomax & CoBliSS & FastICA \\
\hline 1 & $1.4 \pm 4.3$ & $32.0 \pm 14.5$ & $29.4 \pm 15.0$ & $17.1 \pm 8.2$ & $36.9 \pm 12.3$ \\
2 & $1.7 \pm 2.0$ & $26.6 \pm 10.0$ & $24.6 \pm 10.2$ & $16.1 \pm 7.3$ & $29.0 \pm 9.1$ \\
4 & $1.5 \pm 1.8$ & $24.0 \pm 9.0$ & $26.3 \pm 7.2$ & $16.8 \pm 5.7$ & $28.1 \pm 7.5$ \\
8 & $1.3 \pm 1.8$ & $23.3 \pm 8.7$ & $18.2 \pm 8.8$ & $12.5 \pm 6.3$ & $22.2 \pm 8.6$ \\
\hline
\end{tabular}

Table 7.9. CBSS algorithms mean $S I R_{A A}$ in the first environment as a function of $N_{m}$ regardless of $N_{s}$ expressed with one significant decimal.

\subsubsection{Second Environment}

From the results obtained in the first environment, it can be observed that only two of the analyzed algorithms, i.e. TDD and Infomax, accomplish an AA extraction performance close to the one provided by FastICA. Now, In the second environment, the concern is the performance of BSS algorithms when realistic 12-lead ECGs of AF episodes are considered.

The analysis of the first environment served to discard the two algorithms with bad performance in AA extraction, i.e. MBLMS and CoBliSS, for the se- 


\begin{tabular}{l|l|l|l|l|l|}
$N_{s}$ & MBLMS & TDD & Infomax & CoBliSS & FastICA \\
\hline 1 & $0.140 \pm 0.117$ & $0.914 \pm 0.178$ & $0.861 \pm 0.119$ & $0.464 \pm 0.143$ & $0.997 \pm 0.007$ \\
2 & $0.135 \pm 0.089$ & $0.870 \pm 0.227$ & $0.828 \pm 0.140$ & $0.442 \pm 0.167$ & $0.930 \pm 0.158$ \\
4 & $0.103 \pm 0.075$ & $0.843 \pm 0.216$ & $0.811 \pm 0.138$ & $0.623 \pm 0.181$ & $0.937 \pm 0.125$ \\
8 & $0.088 \pm 0.061$ & $0.772 \pm 0.257$ & $0.669 \pm 0.231$ & $0.345 \pm 0.157$ & $0.809 \pm 0.224$ \\
\hline
\end{tabular}

Table 7.10. CBSS algorithms mean $R_{A A_{t}}$ in the first environment as a function of $N_{m}$ regardless of $N_{s}$ expressed with three significant digits.
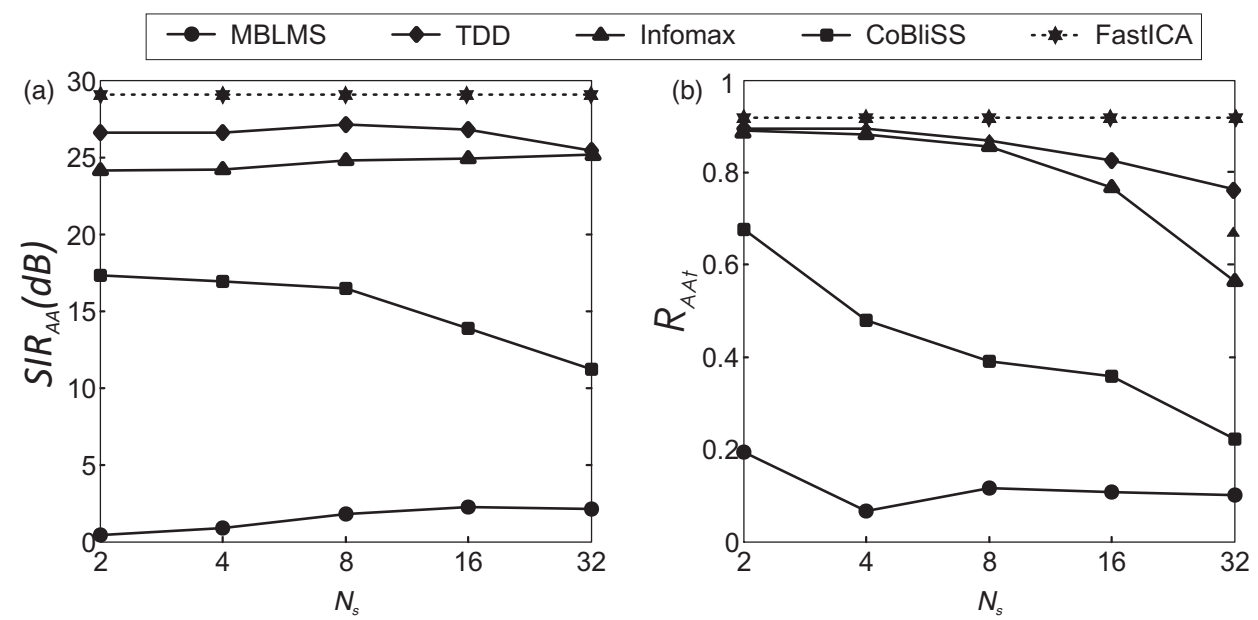

Figure 7.10. CBSS algorithms (a) $S I R_{A A}$ and (b) $R_{A A_{t}}$ mean values in the first environment as a function of the separation matrix filters length $\left(N_{s}\right)$ regardless of the mixing matrix filters length $\left(N_{m}\right)$.

\begin{tabular}{l|l|l|l|l|l|}
$N_{s}$ & MBLMS & TDD & Infomax & CoBliSS & FastICA \\
\hline 2 & $0.4 \pm 0.8$ & $26.6 \pm 9.9$ & $24.1 \pm 9.8$ & $17.3 \pm 7.7$ & $29.1 \pm 9.4$ \\
4 & $0.8 \pm 1.2$ & $26.6 \pm 8.6$ & $24.2 \pm 9.9$ & $16.9 \pm 6.9$ & $29.1 \pm 9.4$ \\
8 & $1.8 \pm 2.9$ & $27.1 \pm 12.1$ & $24.7 \pm 10.3$ & $16.5 \pm 6.8$ & $29.1 \pm 9.4$ \\
16 & $2.2 \pm 3.7$ & $26.8 \pm 11.3$ & $24.9 \pm 10.7$ & $13.9 \pm 6.6$ & $29.1 \pm 9.4$ \\
32 & $2.1 \pm 3.8$ & $25.4 \pm 10.7$ & $25.1 \pm 10.8$ & $11.2 \pm 5.6$ & $29.1 \pm 9.4$ \\
\hline
\end{tabular}

Table 7.11. CBSS algorithms mean $S I R_{A A}$ in the first environment as a function of $N_{S}$ regardless of $N_{m}$ expressed with one significant decimal. 


\begin{tabular}{l|l|l|l|l|l|}
$N_{s}$ & MBLMS & TDD & Infomax & CoBliSS & FastICA \\
\hline 2 & $0.194 \pm 0.139$ & $0.896 \pm 0.151$ & $0.890 \pm 0.159$ & $0.677 \pm 0.196$ & $0.918 \pm 0.128$ \\
4 & $0.066 \pm 0.044$ & $0.895 \pm 0.182$ & $0.883 \pm 0.142$ & $0.480 \pm 0.185$ & $0.918 \pm 0.128$ \\
8 & $0.117 \pm 0.096$ & $0.868 \pm 0.226$ & $0.857 \pm 0.138$ & $0.390 \pm 0.151$ & $0.918 \pm 0.128$ \\
16 & $0.106 \pm 0.076$ & $0.826 \pm 0.251$ & $0.766 \pm 0.162$ & $0.359 \pm 0.161$ & $0.918 \pm 0.128$ \\
32 & $0.101 \pm 0.073$ & $0.763 \pm 0.288$ & $0.564 \pm 0.184$ & $0.222 \pm 0.136$ & $0.918 \pm 0.128$ \\
\hline
\end{tabular}

Table 7.12. CBSS algorithms mean $R_{A A_{t}}$ in the first environment as a function of $N_{s}$ regardless of $N_{m}$ expressed with three significant digits.

cond environment analysis. Therefore, only TDD and Infomax were analyzed in this second environment. In the first environment, a simplified mixing-unmixing model of two sources and two observations is considered. Obviously, this twoby-two model does not suffice in a real AA extraction problem where the number of bioelectric sources is typically greater than two, since the AA, the VA, noise sources, and other bioelectric phenomena are always present. On the other hand, it would be illogical to discard the information brought by the rest of leads that a standard 12-leads ECG comprises. Hence, the CBSS algorithms must be adapted to a 12-by-12 model, that is, a model where twelve observations, i.e. twelve leads, and twelve independent sources can be contemplated.

The adaptation of CBSS algorithms to a 12-by-12 model was not an easy task since all of them were initially developed to solve a 2-by-2 problem. This adaptation had to be carried out subsequently in this work. This justifies the use of the 2-by-2 model in order to discard those algorithms which present a bad extraction performance. The adaptability of the convolutive algorithms to the 12-by-12 model was studied in every CBSS algorithm. It resulted that MBLMS, Infomax and CoBliSS could be adapted by modifying some input features on the available Matlab code. On the contrary, the TDD Matlab is constrained to a 2-by-2 model, and could not be adapted to the 12-by-12 model. As a consequence, in this second environment only the results of the Infomax algorithm are presented, given that is the only tested algorithm that accomplishes a quite good performance and is easily adaptable to the 12-by-12 model at the same time.

Results of the second environment are summarized in Figure 7.11 and in table 7.13, where the values of $S I R_{A A}$ and correlation are presented together. Seven different unmixing FIR filters length, i.e. $N_{s}=2,4,8,16,32,64$ and 128, were considered. Note that the feature $N_{m}$, i.e., the length of the filters of the mixing matrix, does not appear in the table because the mixing process of sources is unknown in this second environment.

Also in this environment, the maximum mean $S I R_{A A}$ obtained by FastICA algorithm $(22.8 \pm 4.9 \mathrm{~dB})$ is several decibels higher than the maximum mean $S I R_{A A}$ obtained by Infomax $(19.8 \pm 6.0 \mathrm{~dB})$. In the same way, the maximum mean $R_{A A}$ obtained by FastICA $(0.839 \pm 0.164)$ is slightly higher than the one obtained by Infomax $(0.765 \pm 0.155)$. In spite of this, it is interesting to note that the highest 

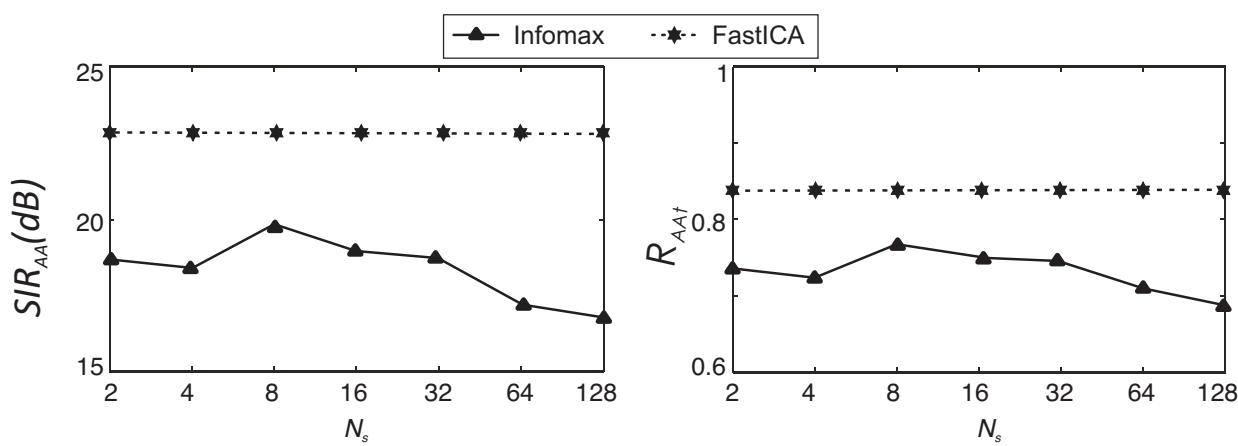

Figure 7.11. $S I R_{A A}$ and $R_{A A}$ mean values of the Infomax algorithm in the second environment as a function of the FIR filters length of the separation matrices $\left(\mathrm{N}_{s}\right)$. Seven different lengths of the filters were considered.

\begin{tabular}{l|c|c|}
$N_{s}$ & $S I R_{A A}(d B)$ & $R_{A A_{t}}$ \\
\hline 2 & $18.7 \pm 5.5$ & $0.737 \pm 0.163$ \\
4 & $18.4 \pm 5.6$ & $0.722 \pm 0.170$ \\
8 & $19.8 \pm 6.0$ & $0.765 \pm 0.155$ \\
16 & $19.0 \pm 5.3$ & $0.750 \pm 0.153$ \\
32 & $18.8 \pm 5.1$ & $0.743 \pm 0.163$ \\
64 & $17.3 \pm 4.9$ & $0.709 \pm 0.131$ \\
128 & $16.8 \pm 5.7$ & $0.688 \pm 0.176$ \\
\hline FastICA & $22.8 \pm 4.9$ & $0.839 \pm 0.164$ \\
\hline
\end{tabular}

Table 7.13. $S I R_{A A}$ and $R_{A A_{t}}$ mean values of the Infomax algorithm as a function of $N_{s}$ in the second environment. 
values of mean $S I R_{A A}$ and mean $R_{A A_{t}}$ of the Infomax algorithm do not occur for $N_{s}=1$ but for $N_{s}=8$. As in the first environment, this fact can be interpreted as a likely hidden convolutive mixture of the cardioelectric real sources that would explain this maximum of performance at $N_{s}=8$. These results encouraged us to continue the research on CBSS algorithms. This research has materialized in the development of a new AA extraction algorithm for convolutive mixtures, the CMBS, the results of which are presented in the next section.

\subsection{CMBS}

In spite of the lower performance of CBSS algorithms in comparison with FastICA, results of the previous section seem to beer out a convolutive factor of the mixing process of cardioelectric sources in AF ECGs, since a slight improvement of AA extraction performance is observed in all CBSS algorithms for some combinations of $N_{m}$ and $N_{s}$ different from the one that characterizes the instantaneous model $\left(N_{m}=1\right.$ and $\left.N_{s}=1\right)$. On the one hand, the CMBS algorithm was developed with the aim to solve the lack of ECG leads in Holter recordings. The number of leads is increased in CMBS by means of the frequency subband decomposition that the Wavelet transform provides. On the other hand, this subband decomposition was also viewed as a way to enhance the convolutive mixture of sources in different frequency bands, taking into account the results of CBSS algorithms. In other words, the aforementioned convolutive factor could be located only in some frequency bands so that a frequency band subdivision of the inputs could benefit the performance of convolutive CBSS algorithms.

In the next subsections, the results of CMBS are presented for both environments introduced in section 6.3. Three levels of noise, i.e. no noise, $15 \mathrm{~dB}$ and $5 \mathrm{~dB}$ of SNR, are presented for every performance index. The extraction performance of CMBS was compared with the performance of WBS and ICA in both scenarios.

\subsubsection{First Environment}

The results of the first environment are shown in Figures 7.12, 7.13, 7.14, and 7.15. All experiments of CMBS were executed for $N_{m}=1$ and $N_{s}=4$, since CMBS is based on the Infomax algorithm, which reaches the best outcomes for these values of $N_{s}$ and $N_{m}$. On the other hand, $N_{m}$ was fixed to one for WBS and FastICA, given that these algorithms follow the instantaneous model and $N_{m}=1$ is optimal in this case. For the same reason, $N_{s}$ had not to be chosen for WBS and FastICA, since feature $N_{s}$ is only meaningful for the instantaneous model.

Figure 7.12 shows the mean $R_{A A_{t}}$ between the original AA and the one extracted by FastICA, WBS and CMBS from the synthetic ECG recordings of the first environment at three levels of SNR. When no noise is added to the signal 
recordings, the mean CMBS $R_{A A_{t}}(0.955 \pm 0.113)$ is very close to he mean FastICA $R_{A A_{t}}(0.997 \pm 0.014)$. This value is very similar to the one obtained by Infomax for $N_{m}=1$ and $N_{s}=4(0.955 \pm 0.091)$. The mean WBS mean $R_{A A_{t}}$ for no noise $(0.880 \pm 0.354)$ is lower than those of CMBS and FastICA. In the same figure, a clear tend can be observed when the additive noise of leads increases or, in other words, the SNR decreases. For a SNR of $15 \mathrm{~dB}$, the highest $R_{A A_{t}}$ is attained by CMBS $(0.936 \pm 0.368)$, followed by WBS $(0.855 \pm 0.360)$ and then by FastICA $(0.563 \pm 0.447)$. It seems that FastICA is the worst affected algorithm by additive noise, maybe as a consequence of the lack of leads. This fact is confirmed when a SNR of $5 \mathrm{~dB}$ is considered. Also in this case, CMBS reaches the highest $R_{A A_{t}}(0.897 \pm 0.318)$, followed by WBS $(0.813 \pm 0.339)$ and FastICA $(0.389 \pm 0.091)$. The convolutive model seems to be more robust against additive noise given that mean CMBS $R_{A A_{t}}$ always exceeds mean WBS $R_{A A_{t}}$.

Similar conclusions can be drawn from the $R_{A A_{f}}$ outcomes presented in Figure 7.13. The mean CMBS $R_{A A_{f}}$ for no noise $(0.931 \pm 0.018)$ is high and very close to mean FastICA $R_{A A_{f}}(0.955 \pm 0.012)$. Again, FastICA is the worst affected algorithm when the level of noise increases. When the SNR is equal to 15 $\mathrm{dB}$, FastICA offers the lowest mean $R_{A A_{f}}(0.709 \pm 0.187)$ and CMBS the highest $(0.924 \pm 0.273)$. This effect is clearer for SNR equal to $5 \mathrm{~dB}$, where the mean $R_{A A_{f}}$ takes values of $0.771 \pm 0.348$ for CMBS, $0.740 \pm 0.331$ for WBS, and $0.602 \pm 0.255$ for FastICA. The intermediate values for WBS at the three levels of noise seem to confirm the deductions from $R_{A A_{t}}$, that is, the convolutive model gives the impression to be more robust against noise than the instantaneous one.

The results for $f_{p 1}$ in the first scenario are shown in Fig 7.14. The mean $f_{p 1}$ estimated by CMBS when no noise is added to signals is $5.49 \pm 1.40 \mathrm{~Hz}$, which is very close to the mean $f_{p 1}$ of the original mixed AA sources, i.e. $5.46 \pm 1.41 \mathrm{~Hz}$. As SNR decreases, the mean $f_{p 1}$ estimated by every extraction algorithm separates from the original $f_{p 1}$. The detrimental effects of noise for a SNR of $15 \mathrm{~dB}$ are worst to the performance of FastICA than to the performance of CMBS or WBS, since the FastICA mean $f_{p 1}$ takes a value of $5.20 \pm 1.37 \mathrm{~Hz}$, which is farther from the original value than the WBS mean $f_{p 1}(5.92 \pm 2.65)$ and the CMBS mean $f_{p 1}$ $(6.07 \pm 1.54)$. Therefore, the CMBS mean $f_{p 1}$ is the less affected by noise for a SNR of $15 \mathrm{~dB}$. When SNR is equal to $5 \mathrm{~dB}$, the worst affected algorithm in terms of $f_{p 1}$ is not FastICA, but WBS instead. Nonetheless, the less affected algorithm is still CMBS, with a mean $f_{p 1}$ of $5.80 \pm 2.25$. These results verify the higher robustness against noise of CMBS in comparison with WBS and FastICA.

As shown in Figure 7.15, also the mean $S C$ of the AA extracted by CMBS for no noise in the first environment $(62.82 \pm 8.01 \%)$ surpasses the results of WBS $(57.00 \pm 1.43 \%)$ and FastICA $(61.78 \pm 6.44 \%)$, in view of the fact that CBSS mean $S C$ is the best approach to the mean $S C$ of the original mixed AA sources $(62.92 \pm$ $11.11 \%)$. The increase of noise provokes a increasing separation of the estimated mean $S C$ from the original mean $S C$. At SNR equal to $15 \mathrm{~dB}$, the CMBS mean $S C$ decreases and takes a value of $61.44 \pm 13.07$, which exceeds the mean WBS $S C(55.71 \pm 14.04 \%)$ and the mean FastICA $S C(60.86 \pm 7.41 \%)$. At SNR equal to 


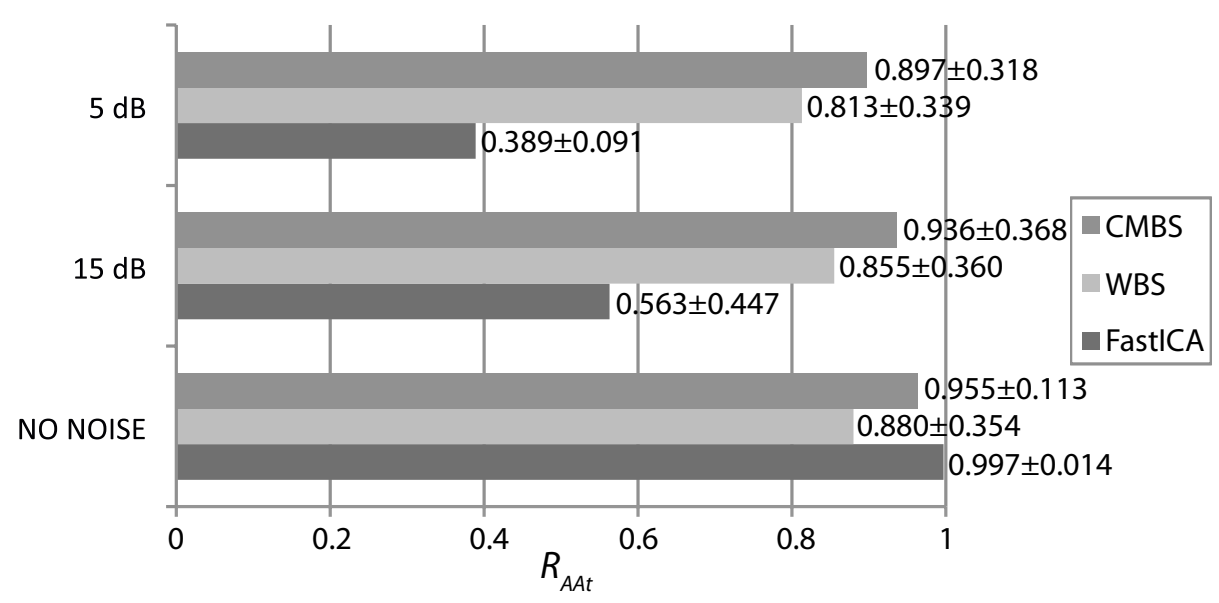

Figure 7.12. Mean $R_{A A_{t}}$ between the original AA and the one extracted by FastICA, WBS and CMBS from synthetic ECG recordings of the first environment at three levels of SNR.

$5 \mathrm{~dB}$, the mean CMBS mean $S C$ falls up to $60.04 \pm 14.16$. Despite this, the CMBS mean $S C$ is greater than the WBS mean $S C(47.61 \pm 14.14)$ and the FastICA mean $S C(44.68 \pm 16.11 \%)$ at this level of noise.

The selection of the optimal wavelet mother function for the CMBS algorithm is elucidated in table 7.14. This table summarizes the results of CMBS when different wavelet mother functions are used to implement the wavelet decomposition stage of the algorithm. The results are given as the mean values of $R_{A A_{t}}, R_{A A_{f}}, f_{p 1}(H z)$ and $S C$ obtained by CMBS in the first environment. Only the values for no noise are tabulated. The table presents the results of the wavelet mother function with best results of each wavelet family. Specifically, these wavelet mother functions are Symmlet(8), Bior(4.4), Coiflet(3) and Haar. All wavelet mother functions optimized the CMBS outcomes when eight decomposition levels were considered. Hence, all results exposed in table 7.14 are computed for this decomposition level. CMBS obtains the highest values of $R_{A A_{t}}$ $(0.955 \pm 0.113)$ and the highest $R_{A A_{f}}(0.931 \pm 0.018)$ when the decomposition stage is implemented with Symlet(8). The mean $R_{A A_{t}}$ and the mean $R_{A A_{f}}$ obtained by CMBS, when the wavelet mother Bior(4.4) is used, approach maximum values but do not reach them. The mean $R_{A A_{t}}$ and the mean $R_{A A_{f}}$ for the rest of wavelet mother functions are farther from the maximum values. With reference to $f_{p}$ and $S C, C M B S$ offers the best approximation to the original values, i.e. $5.46 \pm 1.41 \mathrm{~Hz}$ and $62.92 \pm 11.11 \%$. In short, the best CMBS outcomes are reached when the wavelet mother Symmlet(8) with eight decomposition level is chosen. Consequently, this option was selected for the final design of the CMBS algorithm in both environments. 


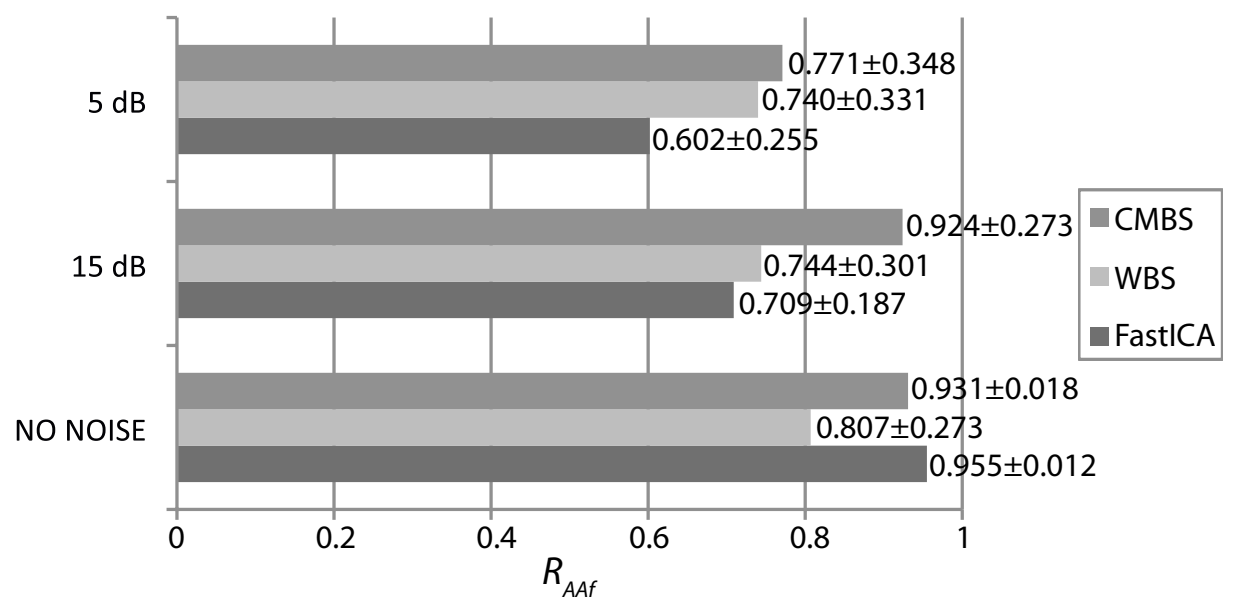

Figure 7.13. Mean $R_{A A_{f}}$ between the original AA and the one extracted by FastICA, WBS and CMBS from synthetic ECG recordings of the first environment at three levels of SNR.

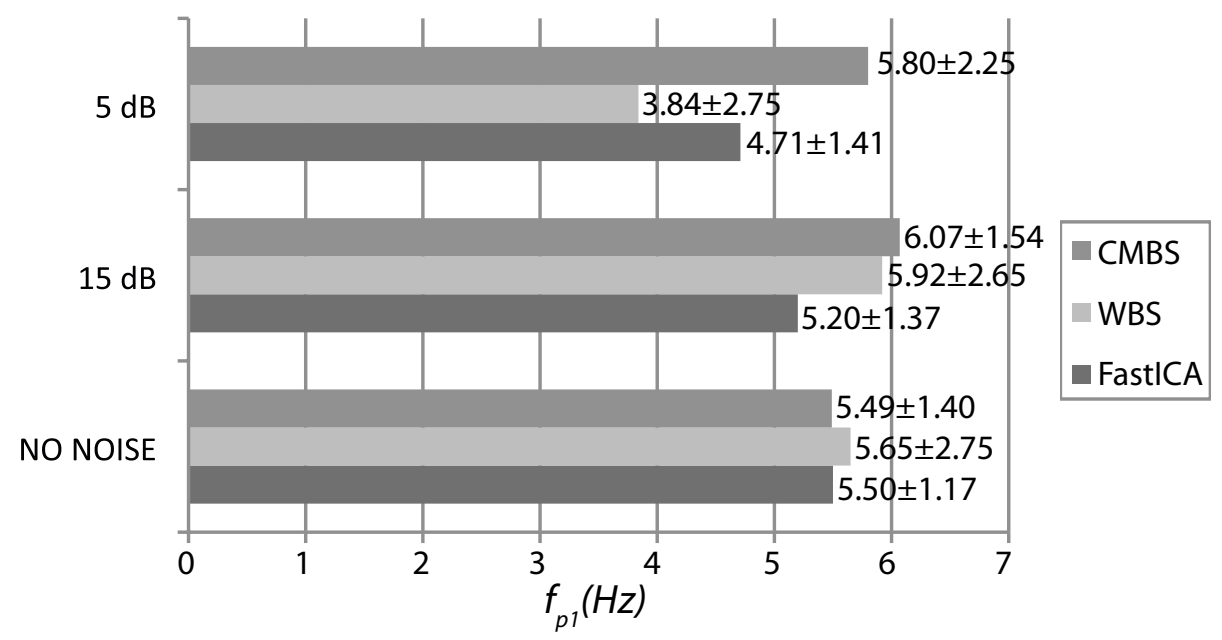

Figure 7.14. Mean $f_{p 1}$ of the AA extracted by FastICA, WBS and CMBS from synthetic ECG recordings of the first environment at three levels of SNR. 


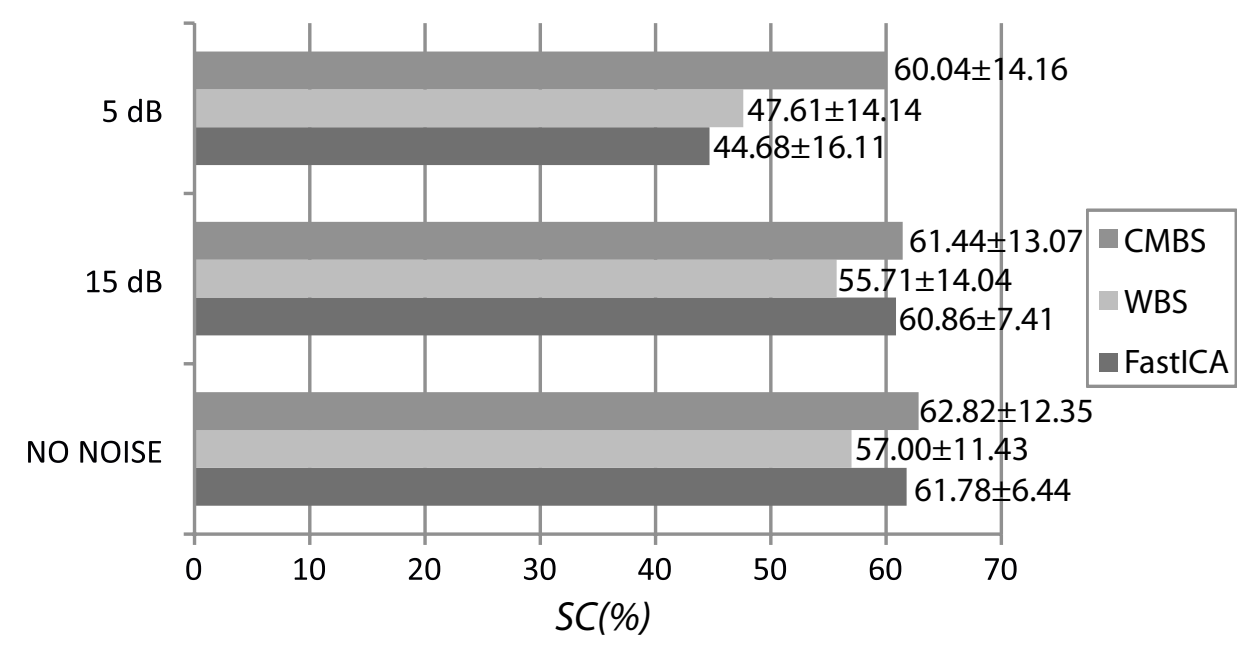

Figure 7.15. Mean $S C$ of the AA extracted by FastICA, WBS and CMBS from synthetic ECG recordings of the first environment at three levels of SNR.

\begin{tabular}{l|c|c|c|c|} 
& $R_{A A_{t}}$ & $R_{A A_{f}}$ & $f_{p 1}(H z)$ & $S C(\%)$ \\
\hline Symmlet 8 & $0.955 \pm 0.113$ & $0.931 \pm 0.018$ & $5.49 \pm 1.40$ & $62.82 \pm 12.35$ \\
Bior 6 & $0.895 \pm 0.021$ & $0.902 \pm 0.321$ & $6.01 \pm 2.16$ & $55.21 \pm 2.55$ \\
Daubechies 10 & $0.825 \pm 0.343$ & $0.880 \pm 0.225$ & $5.11 \pm 1.81$ & $50.34 \pm 10.36$ \\
Coiflet 3 & $0.771 \pm 0.254$ & $0.730 \pm 0.136$ & $4.15 \pm 2.23$ & $43.41 \pm 12.05$ \\
Harr 4 & $0.648 \pm 0.283$ & $0.692 \pm 0.301$ & $4.02 \pm 2.51$ & $39.18 \pm 14.42$ \\
\hline
\end{tabular}

Table 7.14. CMBS mean values of $R_{A A_{t}}, R_{A A_{f}}, f_{p 1}(H z)$ and $S C$ for different wavelet mother functions used in the implementation of the CMBS wavelet decomposition stage. 


\subsubsection{Second Environment}

In this second scenario, where the database consists of real Holter ECG recordings, only $f_{p 1}$ and $S C$ were computed as performance indexes of the AA extraction because the other two indexes could only be calculated for synthetic signals. On the other hand, a comparison with original values cannot be made in the case of real signals and it can be just verified that $f_{p 1}$ and $S C$ are within the expected bounds.

The mean $f_{p 1}$ values of the AA extracted by FastICA, WBS and CMBS from the real ECG recordings of the second environment are shown in Figure 7.16 at three levels of SNR. For no noise, all three algorithms have a mean $f_{p 1}$ in the expected rank from 5 to $8 \mathrm{~Hz}$. In this case, the mean $f_{p 1}$ takes a value of $5.46 \pm 1.47$ for CMBS, $5.24 \pm 2.05$ for WBS and $5.40 \pm 2.08$ for FastICA. Also when SNR is equal to $15 \mathrm{~dB}$, the mean $f_{p 1}$ of every algorithm is within the expected rank. For this level of SNR, the less affected algorithm is CMBS with a mean $f_{p 1}$ equal to $5.21 \pm 1.71$, and the worst affected is WBS with a mean $f_{p 1}$ of $4.52 \pm 1.64$. On the contrary, when SNR is equal to $5 \mathrm{~dB}$, the worst affected algorithm in terms of $f_{p 1}$ is FastICA, although CMBS is again the lowest affected algorithm with a mean $f_{p 1}$ of $5.35 \pm 1.14$.

Figure 7.17 shows the values of $S C$ in the second environment for FastICA, WBS and CMBS at three levels of SNR. When the case of no noise is considered, both CMBS and WBS reach mean $S C$ values that exceed the expected value of $60 \%$ (71.83 \pm 6.25 for CMBS and $67.13 \pm 9.43$ for WBS). On the contrary, the FastICA mean $S C$ takes a value of $43.23 \pm 9.55$. When the level of noise increases, the mean $S C$ decreases for every algorithm, as shown in Figure 7.17. Nevertheless, the highest mean $S C$ is reached by CMBS at the three levels of SNR, what can be seen as a highest accuracy of this algorithm for the AA extraction.

In brief, also in this second scenario, CMBS presents the best robustness against noise, either $f_{p 1}$ or $S C$ is regarded. The convolutive model of CMBS seems to be better adapted to the problem of AA extraction from real Holter ECG recordings than the instantaneous model of WBS. On the other hand, the low values of $S C$ of FastICA may be explained by the lack of inputs to the algorithm, i.e. two leads, in a context of real ECG recordings.

Finally, the otucomes of CMBS in the second environment are compared in the same scenario with the outcomes of ABS, as a widely extended and accepted time-domain technique for AA extraction. A comparison of mean $f_{p 1}$ can be made from the results shown in table 7.15. Very similar mean $f_{p 1}$ are obtained by both algorithms, what confirms the great accuracy of CMBS also for real ECG recordings. On the other hand, the increase of noise affects CMBS the less, since the least change of the mean $f_{p 1}$ is observed for CMBS and the greatest increment the $f_{p 1}$ standard deviation is suffered by ABS. The same lecture can be made as regards mean $S C$, the values of which are shown in table 7.16. 


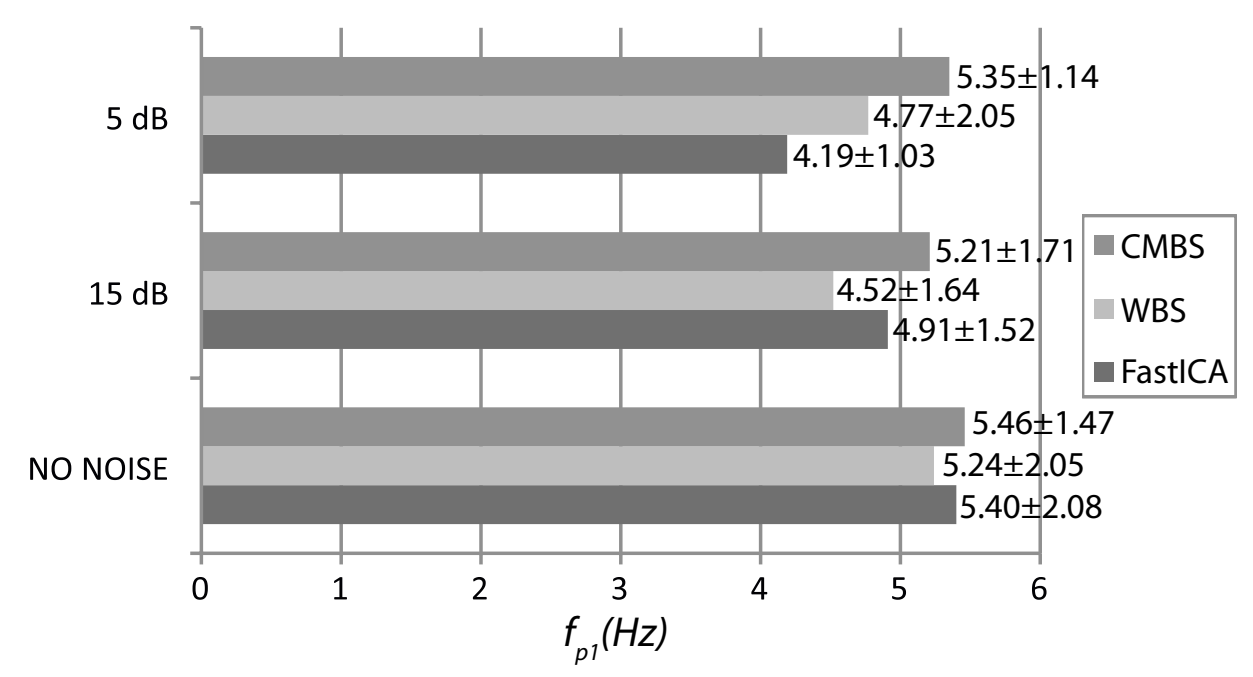

Figure 7.16. Mean $f_{p 1}$ of the AA extracted by FastICA, WBS and CMBS from real ECG recordings of the second environment at three levels of SNR.

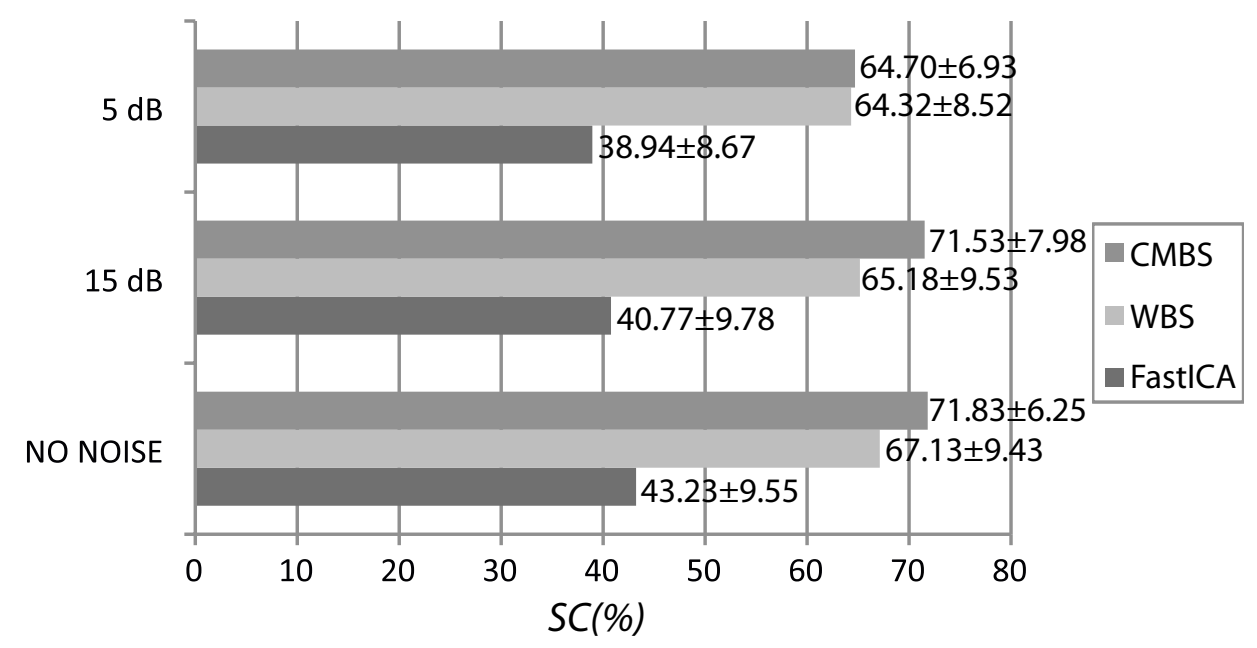

Figure 7.17. Mean $S C$ of the AA extracted by FastICA, WBS and CMBS from real ECG recordings of the second environment at three levels of SNR.

\begin{tabular}{l|c|c|c|} 
& No noise & $15 \mathrm{~dB}$ & $5 \mathrm{~dB}$ \\
\hline CMBS & $5.46 \pm 1.47$ & $5.21 \pm 1.71$ & $5.35 \pm 1.14$ \\
ABS & $5.25 \pm 2.15$ & $6.01 \pm 4.46$ & $6.56 \pm 5.02$ \\
\hline
\end{tabular}

Table 7.15. Mean $f_{p 1}$ of CMBS and ABS algorithms in the second environment. 


\begin{tabular}{l|c|c|c|} 
& No noise & $15 \mathrm{~dB}$ & $5 \mathrm{~dB}$ \\
\hline CMBS & $71.8 \pm 6.25$ & $71.53 \pm 7.98$ & $64.70 \pm 6.93$ \\
ABS & $68.32 \pm 12.02$ & $63.77 \pm 18.55$ & $61.21 \pm 25.37$ \\
\hline
\end{tabular}

Table 7.16. Mean $S C$ of $\mathrm{CMBS}$ and ABS algorithms in the second environment.

\subsection{MPSE}

The results of MPSE are exposed in the three following subsections. The selection of the optimal time-frequency distribution is justified in subsection 7.3.1. Results of the univariate analysis are presented in subsection 7.3.2 and results of the multivariate analysis in subsection 7.3.3.

\subsubsection{Selection of the Time-Frequency Distribution}

The selection of the optimal time-frequency distribution was made with regard to the preliminary study results shown in tables 7.17 and 7.18. Table 7.17 presents the $S a m p E n$ of $f_{p 1}$ and bilateral significance between groups $\mathrm{N}$ and T computed for the nine studied time-frequency distributions. Similarly, the SampEn of $S C$ and its bilateral significance for every time-frequency distribution are shown in table 7.18. The spectrogram was the one that reached the least bilateral significance for both $f_{p 1}$ and $S C$ spectral features, remaining lower than 0.001 . In view of the significance values obtained, the spectrogram was the time-frequency distribution chosen to face up to the rest of the study by considering twelve spectral features.

\begin{tabular}{l|c|c|c} 
t-f distribution & Group N & Group T & Bil. Sig. \\
\hline SP & $0.1311 \pm 0.0314$ & $0.0788 \pm 0.0346$ & $<0.001$ \\
WV & $0.0470 \pm 0.0150$ & $0.0566 \pm 0.0264$ & 0.239 \\
PWV & $0.0514 \pm 0.0182$ & $0.0579 \pm 0.0227$ & 0.394 \\
MH & $0.0360 \pm 0.0054$ & $0.0346 \pm 0.0071$ & 0.533 \\
PMH & $0.0178 \pm 0.0043$ & $0.0121 \pm 0.0051$ & 0.003 \\
PG & $0.0360 \pm 0.0054$ & $0.0346 \pm 0.0071$ & 0.533 \\
PPG & $0.0178 \pm 0.0043$ & $0.0121 \pm 0.0051$ & 0.003 \\
ZAM & $0.0398 \pm 0.0180$ & $0.0375 \pm 0.0169$ & 0.727 \\
CW & $0.0385 \pm 0.0171$ & $0.0352 \pm 0.0156$ & 0.576 \\
\hline
\end{tabular}

Table 7.17. SampEn of $f_{p 1}$ and bilateral significance between groups $\mathrm{N}$ and $\mathrm{T}$ computed for nine time-frequency distributions: spectrogram (SP), Wigner-Ville (WV), pseudo-Wigner-Ville (PWV), Margeneau-Hill (MH), pseudo-Margeneau-Hill (PMH), Page (PG), pseudo-Page (PPG), Zhao-AtlasMarks (ZAM) and Choi-Williams (CW). 


\begin{tabular}{l|c|c|c} 
t-f distribution & Group N & Group T & Bil. Sig. \\
\hline SP & $0.4822 \pm 0.0743$ & $0.4009 \pm 0.0976$ & $<0.001$ \\
WV & $0.2029 \pm 0.0354$ & $0.2297 \pm 0.0568$ & 0.142 \\
PWV & $0.2358 \pm 0.0370$ & $0.2500 \pm 0.0501$ & 0.390 \\
MH & $0.2256 \pm 0.0831$ & $0.2289 \pm 0.0675$ & 0.904 \\
PMH & $0.1932 \pm 0.0445$ & $0.1362 \pm 0.0766$ & 0.024 \\
PG & $0.2256 \pm 0.0831$ & $0.2289 \pm 0.0675$ & 0.904 \\
PPG & $0.1932 \pm 0.0445$ & $0.1362 \pm 0.0767$ & 0.024 \\
ZAM & $0.1493 \pm 0.0472$ & $0.1365 \pm 0.0500$ & 0.484 \\
CW & $0.1443 \pm 0.0313$ & $0.1332 \pm 0.0349$ & 0.368 \\
\hline
\end{tabular}

Table 7.18. SampEn of $S C$ and bilateral significance between groups $\mathrm{N}$ and $\mathrm{T}$ computed for nine time-frequency distributions: spectrogram (SP), Wigner-Ville (WV), pseudo-Wigner-Ville (PWV), Margeneau-Hill (MH), pseudo-Margeneau-Hill (PMH), Page (PG), pseudo-Page (PPG), Zhao-AtlasMarks (ZAM) and Choi-Williams (CW).

\begin{tabular}{c|c|c|c|c|}
\cline { 2 - 5 } & \multicolumn{4}{c|}{$r$} \\
& $0.1 S T D$ & $0.15 S T D$ & $0.2 S T D$ & $0.25 S T D$ \\
\hline $\mathrm{m}=1$ & 0.0058 & 0.0050 & 0.0097 & 0.0094 \\
$\mathrm{~m}=2$ & 0.0075 & 0.0061 & 0.0040 & 0.0010 \\
\hline
\end{tabular}

Table 7.19. SampEn of $f_{p 1}$ bilateral significance for different tested values of $m$ and $r$. The values $m=2$ and $r=0.25 S T D$ are used in the study for SampEn of $f_{p 1}$ regarding to the minimum associated bilateral significance.

\subsubsection{Univariate Analysis}

The results of the $t$-test applied to the SampEn of the numerical series for the learning set are summarized in Figure 7.18. These results reveal that it is possible to distinguish between terminating and non-terminating AF in six of the twelve features, considering a feature to be relevant when its bilateral significance is lower than 0.05. These six relevant features are $f_{p 1}, f_{p 2}, \Delta f_{p}, A_{1}, d_{1}$ and $S C$, which bilateral significances are, respectively, $0.001,0.005,0.003,0.004,0.015$, and 0.001 . These results were computed for $m=2$ and $r=0.25$ because the optimal classification was reached with these values. In order to choose $m$ and $r$, we compared the bilateral significance of every spectral feature for $m=1$ or $m=2$ and $r$ between 0.1 and 0.25 times the standard deviation (STD) of the time-series, as suggested by Pincus [125]. As an example, the SampEn of $f_{p 1}$ bilateral significance for the tested values of $m$ and $r$ is presented in Table 7.19, what justifies the aforementioned choice of $m$ and $r$. For the rest of spectral features, the best outcomes were also achieved for $m=2$ and $r=0.25$ STD. A more generic strategy for optimal selection of $m$ and $r$ is developed in [143].

The area under the Receiver Operative Characteristic (ROC) curve for the 


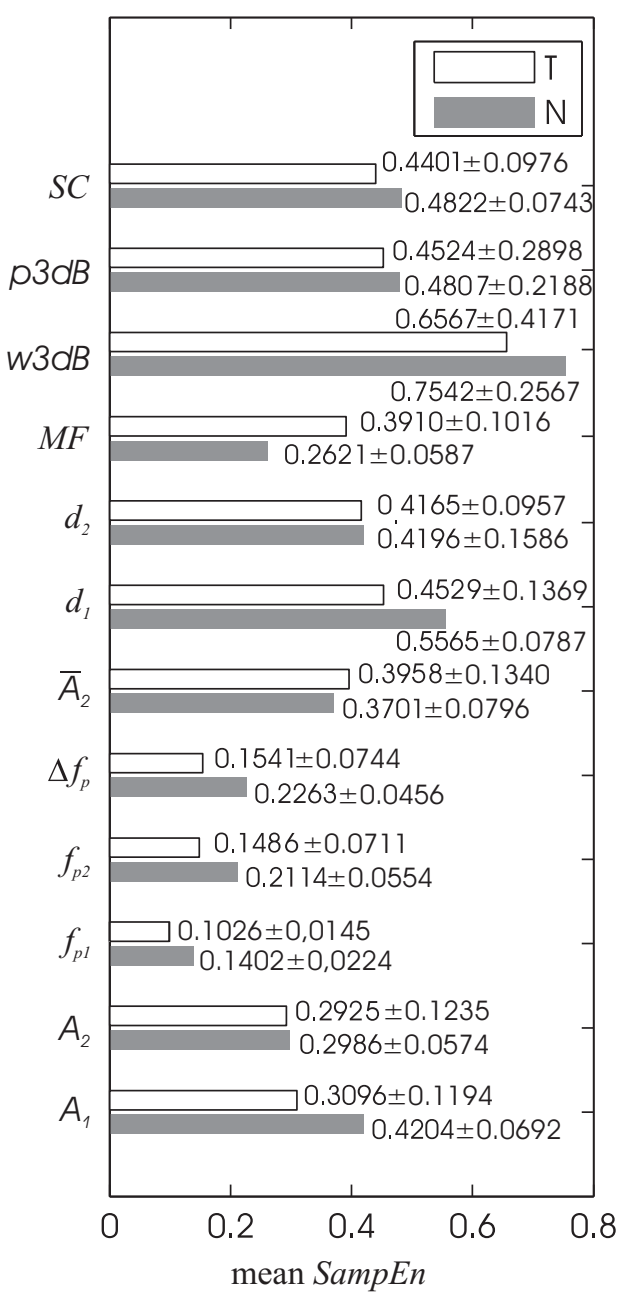

(a)

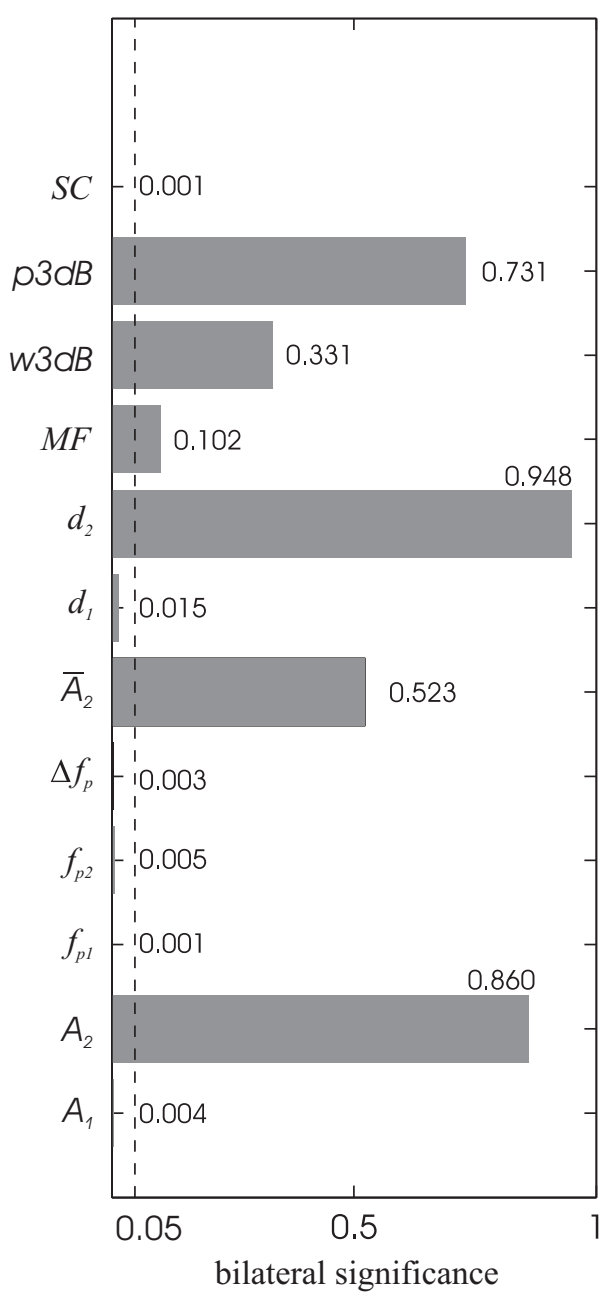

(b)

Figure 7.18. Results of the $t$-test for the $\operatorname{SampEn}$ of all the spectral features. a) Mean and standard deviation of $S a m p E n$ for groups N and T, b) SampEn bilateral significance between groups. A feature is considered relevant when its bilateral significance is lower than 0.05 . 


\begin{tabular}{l|r|r|r|r|r|r} 
& $f_{p 1}$ & $f_{p 2}$ & $\Delta f_{p}$ & $A_{1}$ & $d_{1}$ & $S C$ \\
\hline Area under ROC & $89.7 \%$ & $76.3 \%$ & $77.7 \%$ & $79.9 \%$ & $76.3 \%$ & $72.8 \%$ \\
\hline
\end{tabular}

Table 7.20. Area under ROC curve for the SampEn of the learning set relevant spectral features.

SampEn of the learning set relevant spectral features are shown in table 7.20, where SampEn of $f_{p 1}$ stands out because of its highest value. In the rest of features the bilateral significance obtained is higher than 0.05 , thus they are considered mathematically irrelevant. The mean SampEn in type N recordings is higher than in type $\mathrm{T}$ recordings for all these relevant features. This means that spectral features of $\mathrm{N}$ recordings are less regular than those of $\mathrm{T}$ recordings. An example of this can be seen in Figure 7.19 for feature $f_{p 1}$.
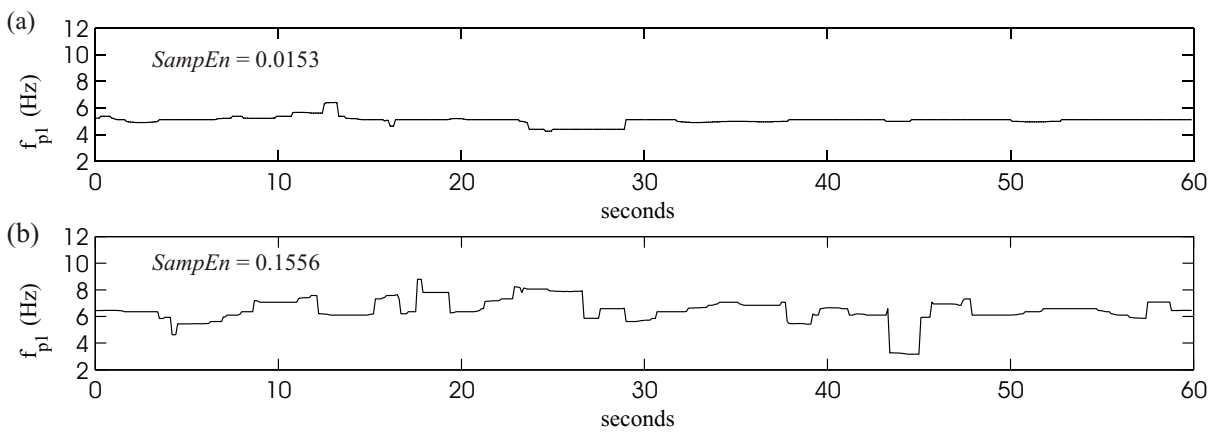

Figure 7.19. a) Type $\mathrm{T}$ and b) type $\mathrm{N} f_{p 1}$ series example with respective $S a m p E n$ values 0.0153 and 0.1556 . Higher SampEn indicates lower regularity of the signal.

The learning set ROC curve for the SampEn of $f_{p 1}$ is depicted in Figure 7.20, which has been fitted using the one-term exponential model. A decision threshold of 0.1173 has been chosen to optimize the result for the learning test. By considering this value of threshold, 19 out of 20 learning recordings have been classified correctly. This decision threshold results from the smoothed ROC curve in a sensitivity of $91 \%$ and 1 - specificity of $14 \%$. Taking the same threshold for the test set, 26 out of 30 recordings have been classified correctly. This resulted in a percentage of recordings properly classified equal to $95 \%$ for the learning set and equal to $86.67 \%$ for the test set. The results obtained by this classification are presented in Figure 7.21 for every recording. The results of this classification can also be viewed in tables 7.21 and 7.22, where misclassified recordings appear highlighted. A higher dispersion of results is observed for the test set than for the learning set. Therefore, we must remark that, despite the difference of dispersion between learning and test sets, the decision threshold of 0.1173 has been correctly chosen. 


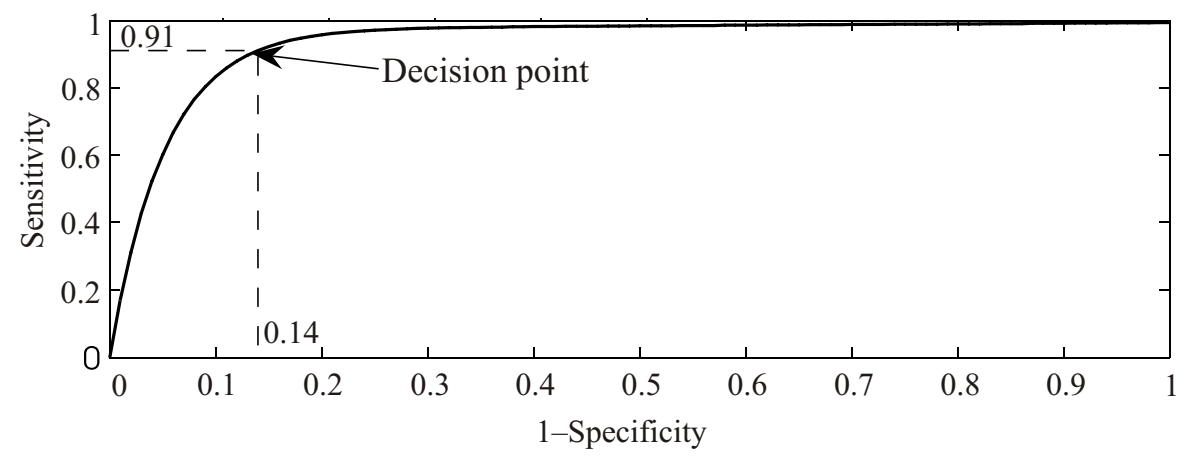

Figure 7.20. Learning set ROC curve fitted using the one-term exponential model for the SampEn of $f_{p 1}$. Decision point chosen for sensitivity $91 \%$ and 1 - specificity $14 \%$.

\begin{tabular}{cc|cc} 
Rec. & SampEn of $f_{p 1}$ & Rec. & SampEn of $f_{p 1}$ \\
\hline$a_{N} 1$ & 0.1256 & $a_{T} 1$ & 0.0928 \\
$a_{N} 2$ & 0.1211 & $a_{T} 2$ & 0.0872 \\
$a_{N} 3$ & 0.1501 & $a_{T} 3$ & 0.1092 \\
$a_{N} 4$ & 0.1333 & $a_{T} 4$ & 0.1114 \\
$a_{N} 5$ & 0.1243 & $a_{T} 5$ & 0.0746 \\
$a_{N} 6$ & 0.1868 & $a_{T} 6$ & 0.1262 \\
$a_{N} 7$ & 0.1319 & $a_{T} 7$ & 0.1118 \\
$a_{N} 8$ & 0.1317 & $a_{T} 8$ & 0.1158 \\
$a_{N} 9$ & 0.1771 & $a_{T} 9$ & 0.1016 \\
$a_{N} 10$ & 0.1206 & $a_{T} 10$ & 0.0955 \\
\hline Mean & 0.1402 & Mean & 0.1026 \\
\hline Std & 0.0224 & Std & 0.0145 \\
\hline
\end{tabular}

Table 7.21. SampEn of $f_{p 1}$ for each recording of the learning set. The $a_{N}$ recordings belong to the nonterminating group, and the $a_{T}$ recordings to the terminating group. A classification was made attending to the threshold value of 0.1173 . Highlighted recordings were misclassified. 


\begin{tabular}{ccc|ccc} 
Rec. & SampEn of $f_{p 1}$ & Original Type & Rec. & SampEn of $f_{p 1}$ & Original Type \\
\hline$b 1$ & 0.1097 & $\mathrm{~N}$ & $b 16$ & 0.1509 & $\mathrm{~N}$ \\
$b 2$ & 0.0778 & $\mathrm{~T}$ & $b 17$ & 0.1217 & $\mathrm{~N}$ \\
$b 3$ & 0.0550 & $\mathrm{~T}$ & $b 18$ & 0.0358 & $\mathrm{~T}$ \\
$b 4$ & 0.1322 & $\mathrm{~N}$ & $b 19$ & 0.1020 & $\mathrm{~T}$ \\
$b 5$ & 0.1265 & $\mathrm{~N}$ & $b 20$ & 0.1566 & $\mathrm{~N}$ \\
$b 6$ & 0.1146 & $\mathrm{~T}$ & $b 21$ & 0.1199 & $\mathrm{~N}$ \\
$b 7$ & 0.1451 & $\mathrm{~N}$ & $b 22$ & 0.1303 & $\mathrm{~N}$ \\
$b 8$ & 0.1540 & $\mathrm{~N}$ & $b 23$ & 0.0767 & $\mathrm{~T}$ \\
$b 9$ & 0.1359 & $\mathrm{~T}$ & $b 24$ & 0.0575 & $\mathrm{~T}$ \\
$b 10$ & 0.1003 & $\mathrm{~N}$ & $b 25$ & 0.1106 & $\mathrm{~T}$ \\
$b 11$ & 0.1147 & $\mathrm{~T}$ & $b 26$ & 0.0645 & $\mathrm{~N}$ \\
$b 12$ & 0.0106 & $\mathrm{~T}$ & $b 27$ & 0.0446 & $\mathrm{~T}$ \\
$b 13$ & 0.1791 & $\mathrm{~N}$ & $b 28$ & 0.1304 & $\mathrm{~N}$ \\
$b 14$ & 0.0744 & $\mathrm{~T}$ & $b 29$ & 0.0945 & $\mathrm{~T}$ \\
$b 15$ & 0.1448 & $\mathrm{~N}$ & $b 30$ & 0.1626 & $\mathrm{~N}$ \\
\hline
\end{tabular}

Table 7.22. SampEn of $f_{p 1}$ for each recording of the test set. A classification was made attending to the threshold value of 0.1173 . Highlighted recordings were misclassified.

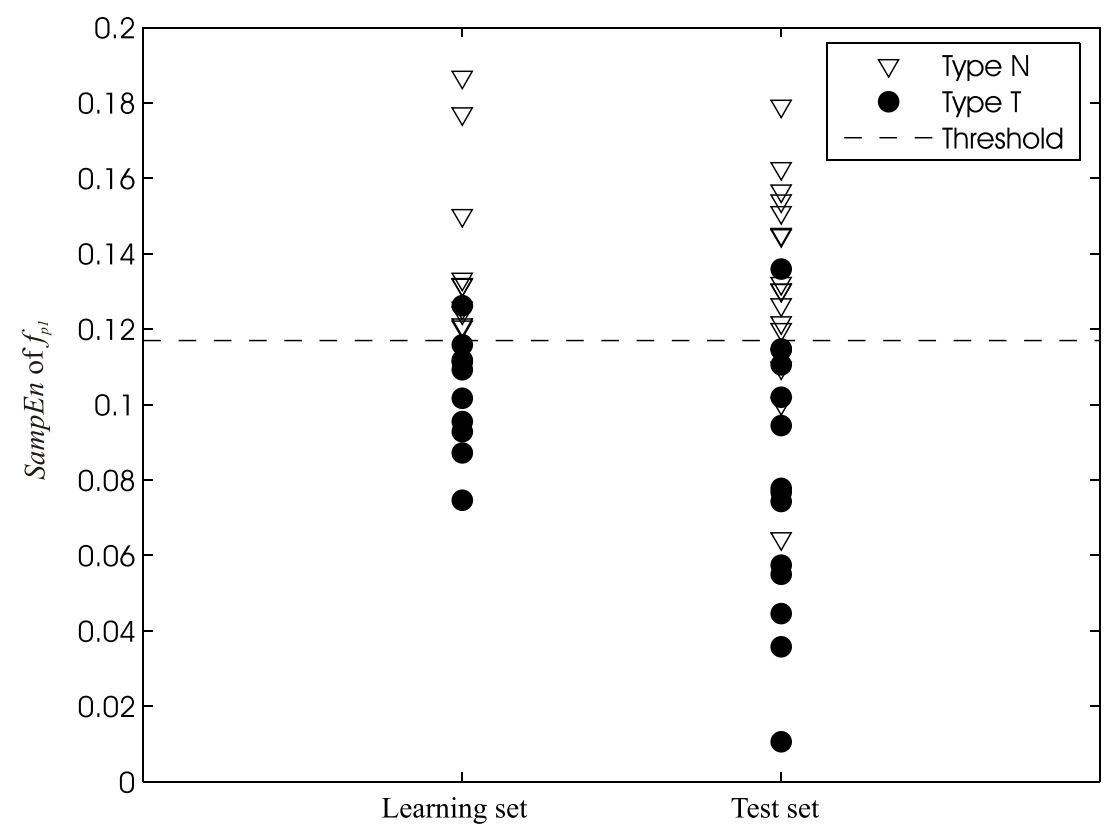

Figure 7.21. Classification of type $\mathrm{N}$ and T episodes using a threshold value for the SampEn of $f_{p 1}$ equal to 0.1173 . The $95 \%$ of the learning set and $86.67 \%$ of the test set recordings were correctly classified. 


\begin{tabular}{rrrrrrrr} 
& $f_{p 1}$ & $f_{p 2}$ & $\Delta f_{p}$ & $A_{1}$ & $d_{1}$ & $S C$ & $A A$ \\
\hline$f_{p 1}$ & 1,000 & $-0,569$ & $-0,446$ & $-0,014$ & $-0,236$ & 0,728 & $-0,384$ \\
$f_{p 2}$ & & 1,000 & 0,902 & 0,035 & 0,279 & $-0,574$ & 0,287 \\
$\Delta f_{p}$ & & & 1,000 & $-0,165$ & 0,126 & $-0,550$ & 0,076 \\
$A_{1}$ & & & & 1,000 & 0,795 & 0,136 & 0,229 \\
$d_{1}$ & & & & & 1,000 & $-0,049$ & 0,293 \\
$S C$ & & & & & & 1,000 & $-0,518$ \\
$A A$ & & & & & & & 1,000
\end{tabular}

Table 7.23. Correlations between the $\operatorname{SanpEn}$ of variables considered in the stepwise analysis.

\subsubsection{Multivariate Analysis}

The previous analysis revealed that the SampEn of the spectral features $f_{p 1}, f_{p 2}$, $\Delta f_{p}, A_{1}, d_{1}$ and $S C$ have a bilateral significance lower than 0.05 and, in consequence, all of them are suitable to be used in discriminant analysis. Subsequently, all these relevant features are considered for the discriminant analysis, being the rest discarded. Furthermore, the $S a m p E n$ of the raw atrial activity was also computed and a mean difference of 0.2508 , with the greatest mean value for the $\mathrm{N}$ group, and a bilateral significance equal to 0.004 were figured out by the $t$-test. This fact suggested to include this feature in the discriminant analysis along with the spectral features. This made possible, in addition, to combine the information obtained from both time and frequency domains. The values $m=2$ and $r=0.25 S T D$ were chosen for the SampEn computation, accordingly to the results of the univariate analysis.

The cross-correlations for the SampEn of spectral significant features and the AA are shown in table 7.23 and depicted as absolute values in Figure 7.22. The highest cross-correlation, 0.902 , occurs between the SampEn of $f_{p 2}$ and $\Delta f_{p}$. Also the pairs of variables $f_{p 1}$ and $S C, A_{1}$ and $d_{1}$ present high values of crosscorrelation, thus it is reasonable to expect in advance that this pairs of variables will not appear in the resultant discriminant model.

The homogeneity of covariance matrices was checked by the Box's $M$ test [144]. Since the significance of this test was low, 0.013, separate groups of covariance matrices were considered for the analysis. The variable selection was performed by forward stepwise analysis and minimization of the Mahalanobis' distance [145]. The process fulfilled by the stepwise analysis is described in table 7.24. This table display the variables that are in the analysis at each step.

The criterion carried out to select the variable to be added to the model in the next step is that this variable has the largest statistic $F$, with a minimum value equal to 3.84. The process was completed in three steps. The SampEn of $f_{p 1}, \Delta f_{p}$ and $A A$ were added to the model by the discriminant analysis, being the rest of features discarded because of their low $F$. As expected, none of the 

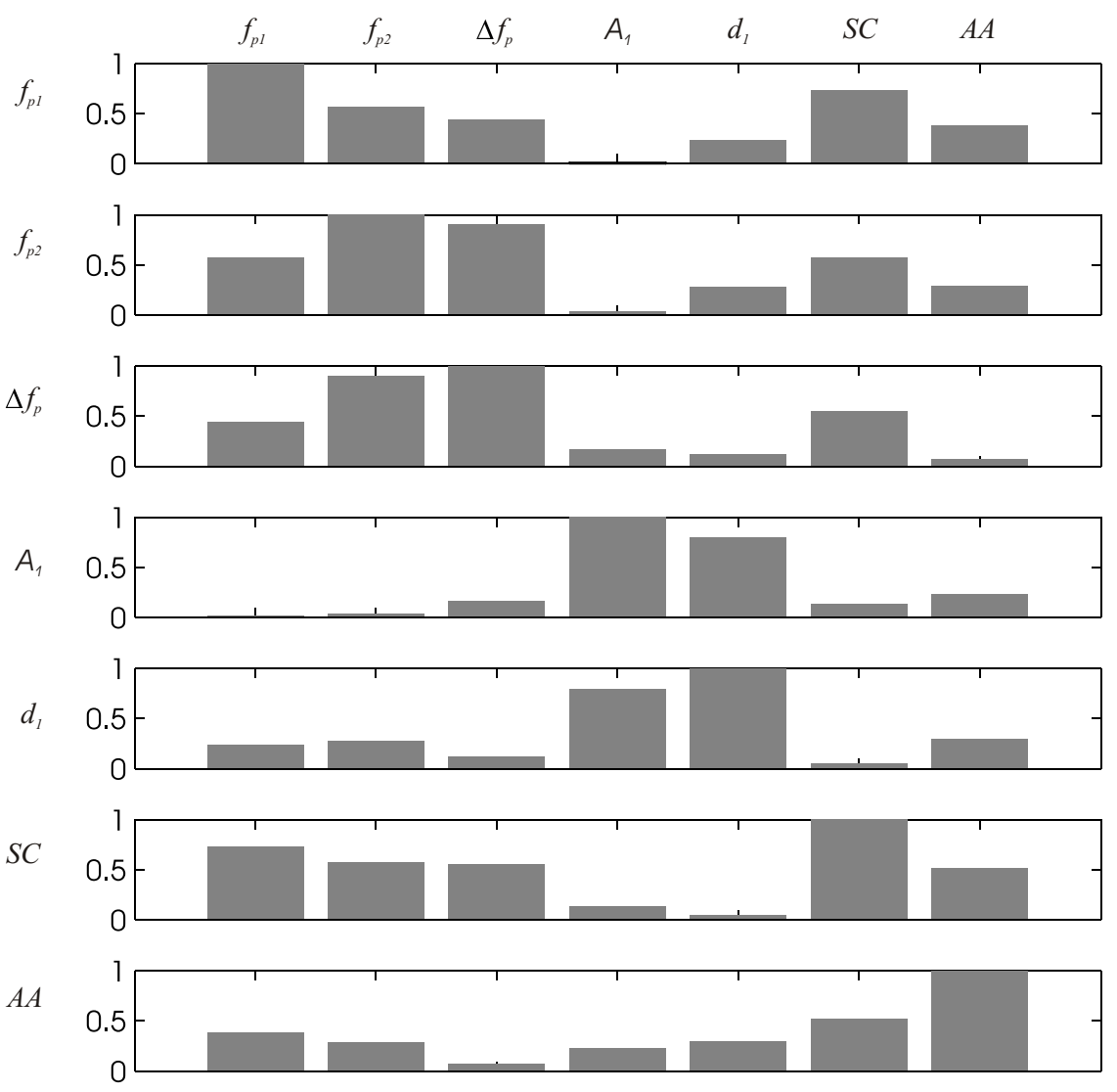

Figure 7.22. Cross-correlation absolute values for the SampEn of the spectral significant features and the AA. Pairs of variables with high cross-correlation do not contribute to the resultant discriminant model. 


\begin{tabular}{c|c|c|c} 
Step & & Tolerance & $F$ to Remove \\
\hline 1 & $A A$ & 1,000 & 5,272 \\
\hline 2 & $A A$ & 0,410 & 26,686 \\
& $f_{p 1}$ & 0,410 & 23,965 \\
\hline 3 & $A A$ & 0,321 & 35,491 \\
& $f_{p 1}$ & 0,259 & 39,760 \\
& $\Delta f_{p}$ & 0,627 & 7,138 \\
\hline
\end{tabular}

Table 7.24. Variables in the stepwise analysis. The statistic $F$ indicates which variable must be added to the model in each step.

\begin{tabular}{l|c} 
& Canonical coefficient \\
\hline SampEn of $f_{p 1}$ & 1,880 \\
SampEn of $A A$ & 1,662 \\
SampEn of $\Delta f_{p}$ & 0,816 \\
\hline
\end{tabular}

Table 7.25. Standardized canonical discriminant function coefficients from the stepwise analysis.

aforementioned pairs of variables with high cross-correlation appears in the final discriminant model. The variables in the stepwise analysis at each step are shown in table 7.24. This kind of multivariate analysis has been previously applied to the study of AF cardioversion [146].

The discriminant function is a plane given by the equation $x_{3}=-0.0355 \cdot x_{1}-$ $0.3380 \cdot x_{2}+0.2065$, where $x_{1}, x_{2}$ and $x_{3}$ represent the SampEn of $f_{p 1}, \Delta f_{p}$ and the AA, respectively. The standardized canonical coefficients of the discriminant function are 1.880 for $x_{1}, 0.816$ for $x_{2}$, and 1.662 for $x_{3}$. The standardized canonical coefficients of the discriminant function are presented in table 7.25, where they are ordered by their importance in the discriminant function. The discriminant function is depicted in Figure 7.23 along with the SampEn values of $f_{p 1}, \Delta f_{p}$, and $A A$ for each recording. These values are tabulated in table 7.26 for learning recordings and in table 7.27 for test recordings. The two misclassified recordings are highlighted. Only two of the recordings were misclassified by this function. A small value of Wilk's lambda test [144] significance $(p<0.001)$ was obtained, which indicates the great discriminatory ability of the function. All of the cases used to create the model, i.e. the learning set, were correctly classified. Regarding the test set, 15 out of 16 type $\mathrm{N}$ cases and 13 out of 14 type T cases were correctly classified (see Table 7.28). Expressing this results in percentages, the $100 \%$ of the learning set recordings were classified correctly. In the test test, $93.75 \%$ of $\mathrm{N}$ recordings and and $92.86 \%$ of $\mathrm{T}$ recordings were classified correctly. The global percentage of test recordings properly classified was $93.33 \%$.

The method was also tested when the SampEn of the AA was excluded from the discriminant analysis. Under these conditions, the discriminant analysis did 
not improved t-test results, what suggested combining time and frequency domains.
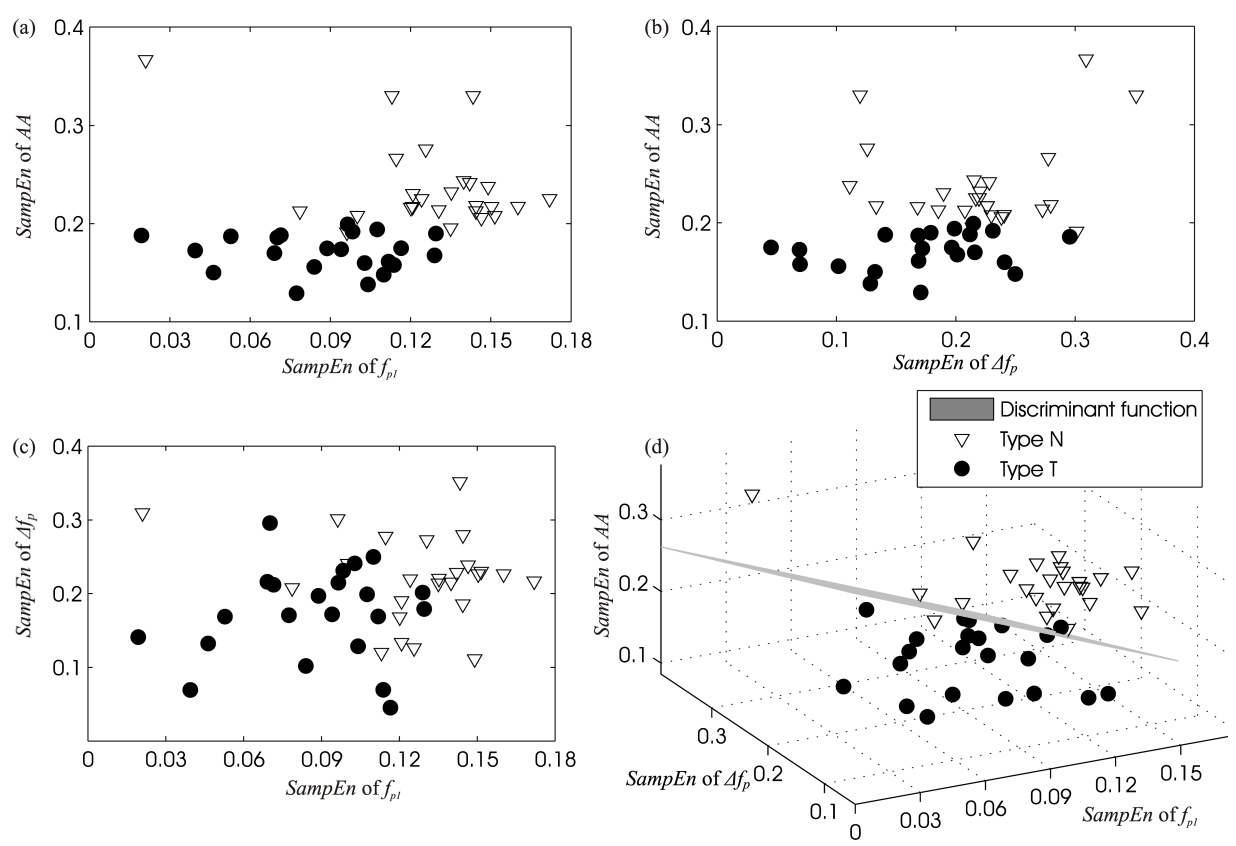

Figure 7.23. Two dimensional representations of the results taken by $\operatorname{SampEn}$ pairs of a) $A A$ and $f_{p 1}$, b) $A A$ and $\Delta f_{p}$, c) $f_{p 1}$ and $\Delta f_{p}$, and 3-D plot showing the hyperplane defined by the discriminant function. The $100 \%$ of the learning set recordings are classified correctly. In the test test, $93.75 \%$ of $\mathrm{N}$ recordings and $92.86 \%$ of $\mathrm{T}$ recordings are correctly classified. 


\begin{tabular}{c|c|c|c} 
Rec. & SampEn of $f_{p 1}$ & SampEn of $\Delta f_{p}$ & SampEn of $A A$ \\
\hline$a_{N} 1$ & 0.1256 & 0.2281 & 0.2993 \\
$a_{N} 2$ & 0.1211 & 0.1333 & 0.2671 \\
$a_{N} 3$ & 0.1501 & 0.1791 & 0.3112 \\
$a_{N} 4$ & 0.1333 & 0.2167 & 0.3404 \\
$a_{N} 5$ & 0.1243 & 0.2202 & 0.2907 \\
$a_{N} 6$ & 0.1868 & 0.2195 & 0.2923 \\
$a_{N} 7$ & 0.1319 & 0.1689 & 0.2750 \\
$a_{N} 8$ & 0.1317 & 0.1967 & 0.2845 \\
$a_{N} 9$ & 0.1771 & 0.2153 & 0.2890 \\
$a_{N} 10$ & 0.1206 & 0.1575 & 0.2716 \\
\hline$a_{T} 1$ & 0.0928 & 0.3512 & 0.3198 \\
$a_{T} 2$ & 0.0872 & 0.3091 & 0.1276 \\
$a_{T} 3$ & 0.1092 & 0.1719 & 0.2305 \\
$a_{T} 4$ & 0.1114 & 0.1260 & 0.1987 \\
$a_{T} 5$ & 0.0746 & 0.1988 & 0.1885 \\
$a_{T} 6$ & 0.1262 & 0.2159 & 0.2516 \\
$a_{T} 7$ & 0.1118 & 0.2498 & 0.2234 \\
$a_{T} 8$ & 0.1158 & 0.1837 & 0.1562 \\
$a_{T} 9$ & 0.1016 & 0.2310 & 0.1489 \\
$a_{T} 10$ & 0.0955 & 0.1383 & 0.1537 \\
\hline
\end{tabular}

Table 7.26. $S a m E n$ values of $f_{p 1}, \Delta f_{p}$, and $A A$ for each recording of the learning set used in the discriminant analysis. 


\begin{tabular}{|c|c|c|c|c|}
\hline Rec. & SampEn of $f_{p 1}$ & SampEn of $\Delta f_{p}$ & SampEn of $A A$ & Original Type \\
\hline$b 1$ & 0.1097 & 0.2263 & 0.1764 & $\mathrm{~N}$ \\
\hline$b 2$ & 0.0778 & 0.1323 & 0.1746 & $\mathrm{~T}$ \\
\hline$b 3$ & 0.0550 & 0.1686 & 0.2278 & $\mathrm{~T}$ \\
\hline$b 4$ & 0.1322 & 0.2383 & 0.299 & $\mathrm{~N}$ \\
\hline 65 & 0.1265 & 0.1111 & 0.2505 & $\mathrm{~N}$ \\
\hline$b 6$ & 0.1146 & 0.2148 & 0.1436 & $\mathrm{~T}$ \\
\hline$b 7$ & 0.1451 & 0.2726 & 0.3101 & $\mathrm{~N}$ \\
\hline$b 8$ & 0.1540 & 0.1680 & 0.2707 & $\mathrm{~N}$ \\
\hline$b 9$ & 0.1359 & 0.1706 & 0.2703 & $\mathrm{~T}$ \\
\hline$b 10$ & 0.1003 & 0.1897 & 0.2791 & $\mathrm{~N}$ \\
\hline$b 11$ & 0.1147 & 0.1446 & 0.1773 & $\mathrm{~T}$ \\
\hline$b 12$ & 0.0106 & 0.2410 & 0.1923 & $\mathrm{~T}$ \\
\hline$b 13$ & 0.1791 & 0.3011 & 0.3222 & $\mathrm{~N}$ \\
\hline$b 14$ & 0.0744 & 0.0691 & 0.1884 & $\mathrm{~T}$ \\
\hline$b 15$ & 0.1448 & 0.1854 & 0.2801 & $\mathrm{~N}$ \\
\hline$b 16$ & 0.1509 & 0.2795 & 0.3078 & $\mathrm{~N}$ \\
\hline$b 17$ & 0.1217 & 0.2138 & 0.2843 & $\mathrm{~N}$ \\
\hline$b 18$ & 0.0358 & 0.0453 & 0.2114 & $\mathrm{~T}$ \\
\hline$b 19$ & 0.1020 & 0.2013 & 0.2435 & $\mathrm{~T}$ \\
\hline$b 20$ & 0.1566 & 0.1198 & 0.2572 & $\mathrm{~N}$ \\
\hline$b 21$ & 0.1199 & 0.2076 & 0.2816 & $\mathrm{~N}$ \\
\hline$b 22$ & 0.1303 & 0.2405 & 0.2931 & $\mathrm{~N}$ \\
\hline$b 23$ & 0.0767 & 0.0695 & 0.2060 & $\mathrm{~T}$ \\
\hline$b 24$ & 0.0575 & 0.1409 & 0.1874 & $\mathrm{~T}$ \\
\hline$b 25$ & 0.1106 & 0.1284 & 0.2207 & $\mathrm{~T}$ \\
\hline$b 26$ & 0.0645 & 0.2774 & 0.3082 & $\mathrm{~N}$ \\
\hline$b 27$ & 0.0446 & 0.1018 & 0.1312 & $\mathrm{~T}$ \\
\hline$b 28$ & 0.1304 & 0.2263 & 0.2898 & $\mathrm{~N}$ \\
\hline$b 29$ & 0.0945 & 0.2956 & 0.2457 & $\mathrm{~T}$ \\
\hline$b 30$ & 0.1626 & 0.2299 & 0.2936 & $\mathrm{~N}$ \\
\hline
\end{tabular}

Table 7.27. SamEn values of $f_{p 1}, \Delta f_{p}$, and $A A$ for each recording of the test set used in the discriminant analysis. Highlighted recordings were misclassified. 


\begin{tabular}{llr|rr|r|}
\hline & & Original Type & \multicolumn{2}{|c|}{ Predicted Type } & Total \\
& & & $\mathrm{N}$ & $\mathrm{T}$ & \\
\hline Learning cases & Count & $\mathrm{N}$ & 10 & 0 & 10 \\
& & $\mathrm{~T}$ & 0 & 10 & 10 \\
\cline { 2 - 6 } & $\%$ & $\mathrm{~N}$ & 100 & 0 & 100 \\
& & $\mathrm{~T}$ & 0 & 100 & 100 \\
\hline Test cases & Count & $\mathrm{N}$ & 15 & 1 & 16 \\
& & $\mathrm{~T}$ & 1 & 13 & 14 \\
\cline { 2 - 6 } & $\%$ & $\mathrm{~N}$ & 93,75 & 6,25 & 100 \\
& & $\mathrm{~T}$ & 7,14 & 92,86 & 100 \\
\hline
\end{tabular}

Table 7.28. Classification of learning and test AF ECG recordings as type $\mathrm{N}$ or type $\mathrm{T}$ by using the discriminant analysis. 


\subsection{Quantization Effects}

Figure 7.24 illustrates the mean $R_{q}$, which represents the correlation between the original 16-bit AA, $A A_{16}$, and the $A A_{q}$ encoded with 4 to 15 bits. Furthermore, the mean $R_{q}$ is also shown in table 7.29 along with the STD. $R_{q}$ was computed for both the raw $A A_{q}$ and the low pass filtered $A A_{q}$. It can be observed the better estimation quality when $R_{A A}$ is applied to the low pass filtered $A A_{q}$. In consequence, the subsequent computing of MAE is applied to the filtered signals. Results reveal that $R_{A A}$ remains higher than 0.7 when the number of encoding bits is higher or equal to nine. For eight or seven bits, $R_{A A}$ approximates to 0.65 , decreasing significantly when the number of bits is equal to or lower than six.

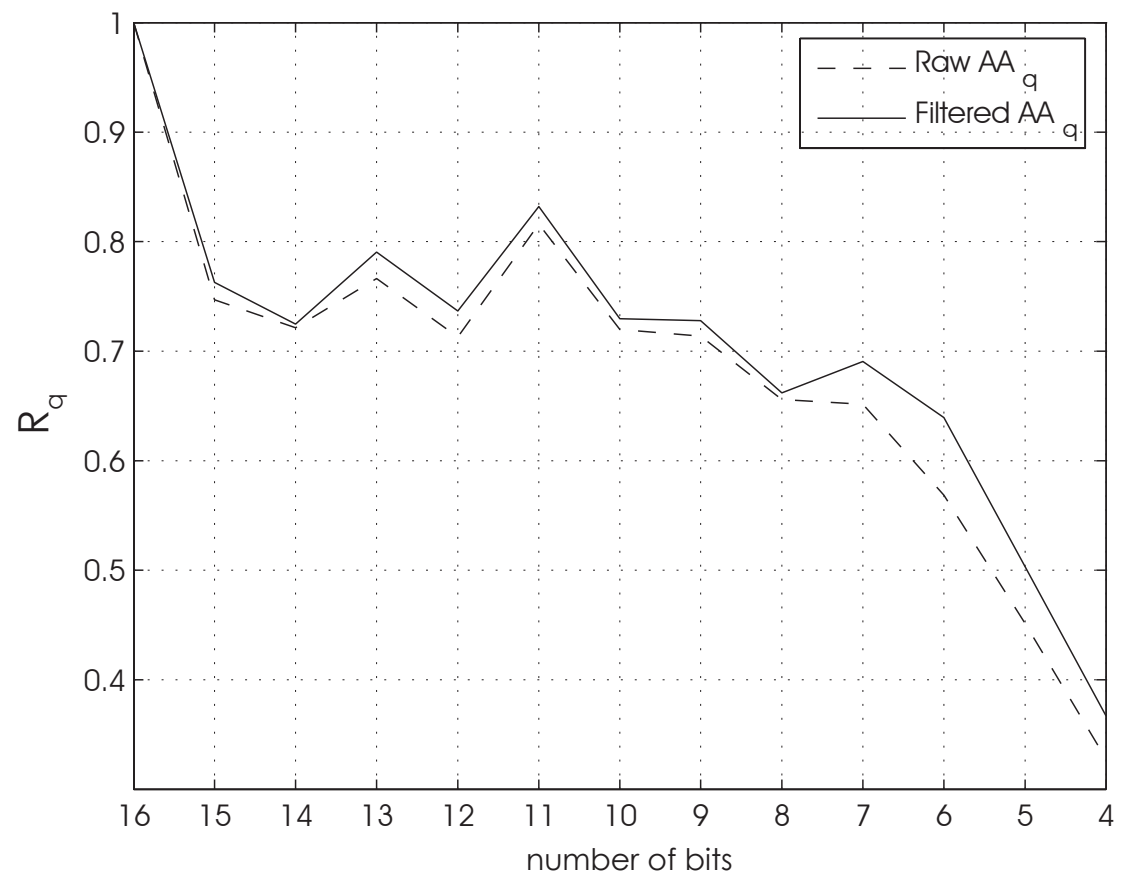

Figure 7.24. $R_{q}$ between the 16-bit AA signal $A A_{16}$ and $A A_{q}$ encoded with 4 to 15 bits. Results are given for non-filtered $A A_{q}$ and for low-pass filtered $A A_{q}$.

On the other hand, the MAE obtained for $f_{p 1}$ is represented in figure 7.25. It can be appreciated that MAE of $f_{p 1}$ does not exceed $1.3 \mathrm{~Hz}$ when the number of bits is higher or equal to seven but it increases drastically below seven bits. In the same way, as shown in figure 7.26, MAE of $S C$ remains lower than $6 \%$ when at least eight encoding bits are used, but higher values of $M A E$ are obtained when a more reduced number of bits are considered. Low values of MAE denote a good approximation of features. Conversely, high values of MAE indicate a bad approximation of features.

Finally, MAE of AA SampEn signals is depicted if figure 7.27. MAE of SampEn 


\begin{tabular}{c|c|c|}
\cline { 2 - 3 } Encoding bits & \multicolumn{2}{|c|}{$R_{q}$} \\
\hline 4 & $0.328 \pm 0.158$ & $0.367 \pm 0.173$ \\
5 & $0.451 \pm 0.207$ & $0.503 \pm 0.219$ \\
6 & $0.569 \pm 0.228$ & $0.639 \pm 0.225$ \\
7 & $0.652 \pm 0.298$ & $0.691 \pm 0.295$ \\
8 & $0.656 \pm 0.284$ & $0.662 \pm 0.301$ \\
9 & $0.714 \pm 0.252$ & $0.728 \pm 0.241$ \\
10 & $0.720 \pm 0.264$ & $0.730 \pm 0.281$ \\
11 & $0.816 \pm 0.202$ & $0.832 \pm 0.193$ \\
12 & $0.713 \pm 0.311$ & $0.737 \pm 0.287$ \\
13 & $0.767 \pm 0.282$ & $0.790 \pm 0.259$ \\
14 & $0.722 \pm 0.306$ & $0.725 \pm 0.326$ \\
15 & $0.747 \pm 0.285$ & $0.763 \pm 0.279$ \\
\hline
\end{tabular}

Table 7.29. Mean $R_{q}$ between $A A_{16}$ and the $A A_{q}$ encoded with 4 to 15 bits. Results are given for non-filtered $A A_{q}$ and for low-pass filtered $A A_{q}$.

denotes a quite good approximation of SampEn when seven or more bits are used with values lower than 0.04 . The graph does not clarify which is the optimum number of bits between 7 an 15 . Nonetheless, it is clearly shown that MAE of $A A$ SampEn increases considerably for a number of bits lower than seven. The values drawn in Figure 7.25, 7.26, and 7.27 are also tabulated in table 7.30 along with the STD. 


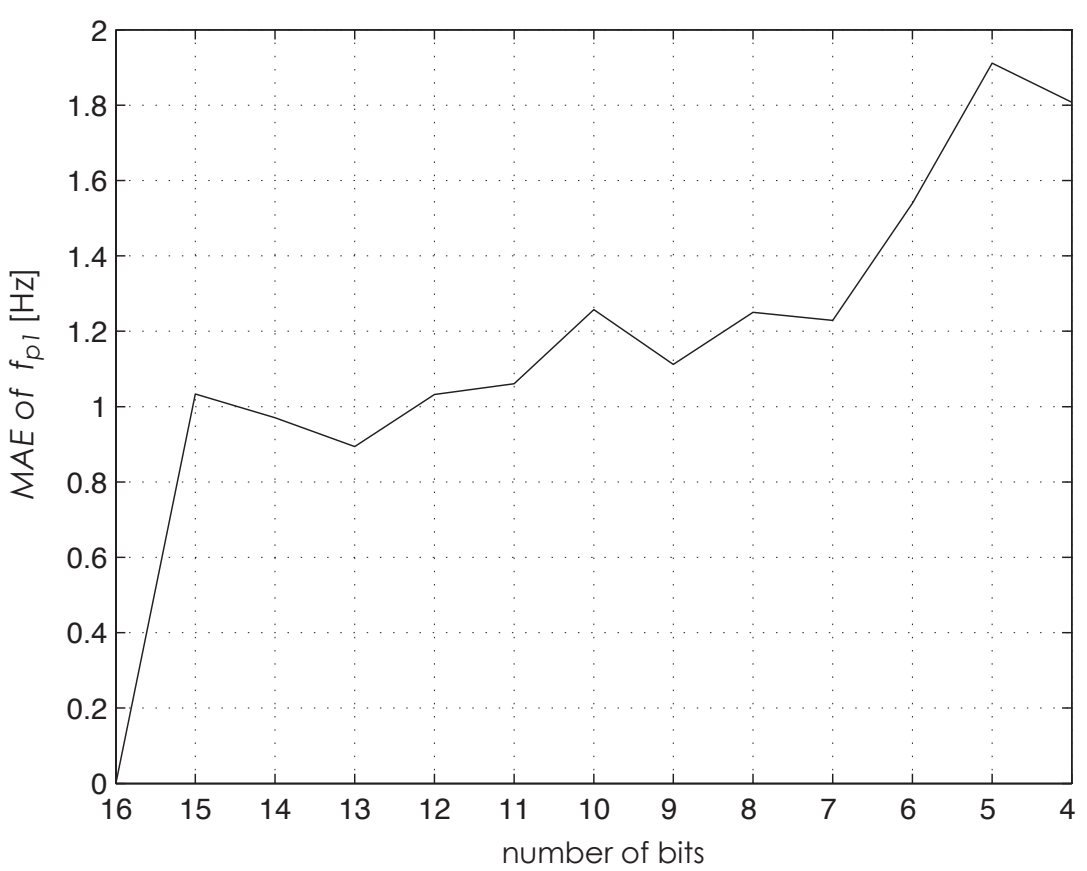

Figure 7.25. Mean MAE of $f_{p 1}$ for a number of encoding bits from 4 to 15 . Higher values of MAE denote a worse approximation of the spectral feature $f_{p 1}$.

\begin{tabular}{c|c|c|c|} 
Enconding bits & MAE of $f_{p 1}$ & MAE of $S C$ & MAE of AA SampEn \\
\hline 4 & $1.67 \pm 0.77$ & $8.36 \pm 2.63$ & $0.0573 \pm 0.0683$ \\
5 & $1.73 \pm 0.90$ & $8.07 \pm 2.31$ & $0.0419 \pm 0.0290$ \\
6 & $1.40 \pm 0.99$ & $6.56 \pm 2.77$ & $0.0432 \pm 0.0289$ \\
7 & $1.10 \pm 0.86$ & $6.20 \pm 3.49$ & $0.0349 \pm 0.0369$ \\
8 & $1.15 \pm 0.94$ & $5.50 \pm 2.66$ & $0.0325 \pm 0.0337$ \\
9 & $1.05 \pm 0.95$ & $4.84 \pm 2.79$ & $0.0277 \pm 0.0391$ \\
10 & $1.18 \pm 1.16$ & $4.89 \pm 3.22$ & $0.0227 \pm 0.0262$ \\
11 & $0.95 \pm 0.81$ & $4.35 \pm 2.44$ & $0.0287 \pm 0.0398$ \\
12 & $0.94 \pm 1.00$ & $4.72 \pm 3.84$ & $0.0216 \pm 0.0269$ \\
13 & $0.82 \pm 0.85$ & $4.59 \pm 3.61$ & $0.0233 \pm 0.0355$ \\
14 & $0.91 \pm 0.84$ & $4.92 \pm 3.93$ & $0.0330 \pm 0.0405$ \\
15 & $1.02 \pm 0.99$ & $4.51 \pm 2.86$ & $0.0352 \pm 0.0463$ \\
\hline
\end{tabular}

Table 7.30. Mean MAE of $f_{p 1}$, SC, and AA SampEn for $A A_{q}$ encoded with 4 to 15 bits. 


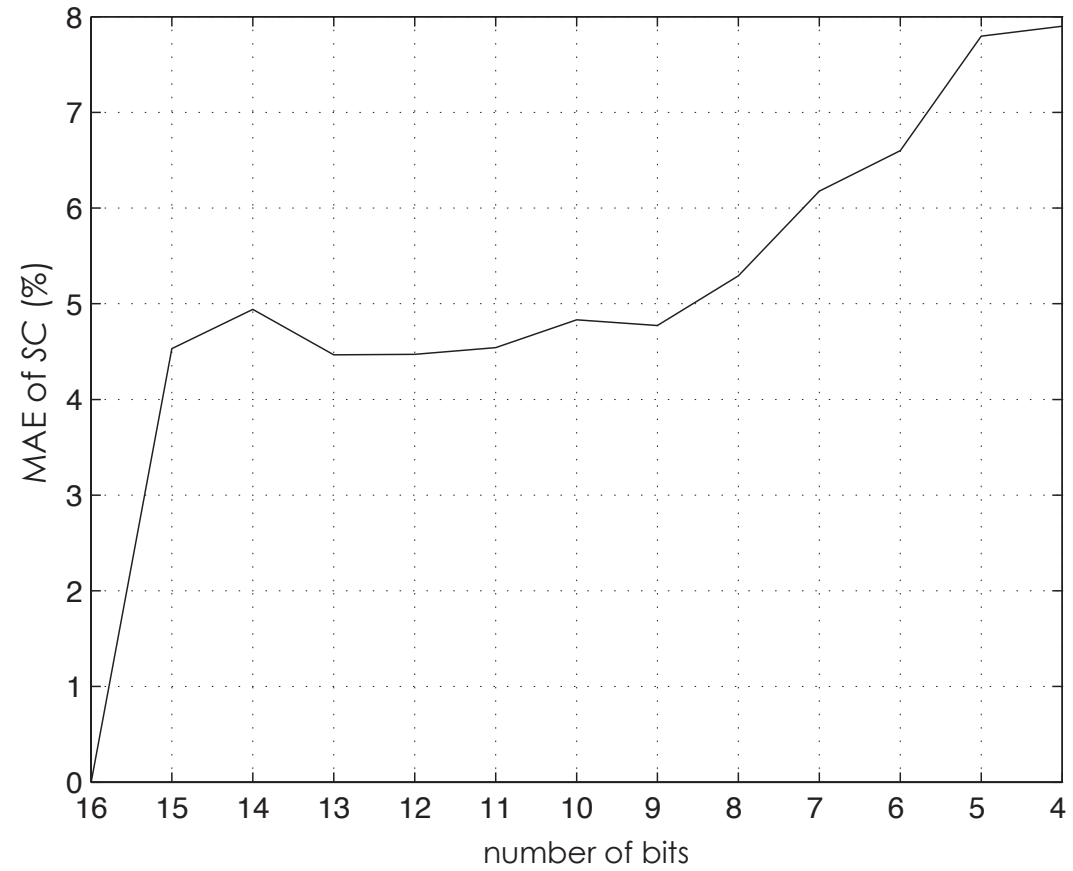

Figure 7.26. Mean MAE of $S C$ for a number of encoding bits from 4 to 15 . Higher values of MAE denote a worse approximation of the spectral feature $S C$.

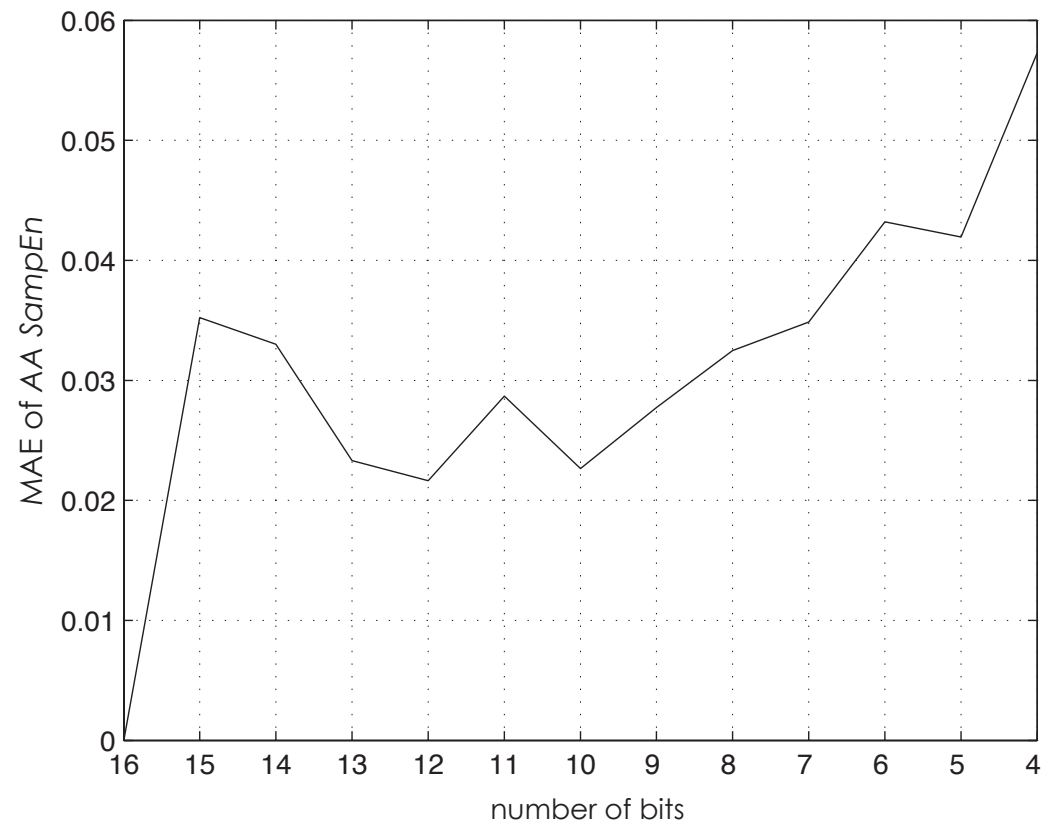

Figure 7.27. Mean $M A E$ of $A A S a m p E n$ for a number of encoding bits from 4 to 15 . Higher values of MAE denote a worse approximation of the AA SampEn. 


\subsection{Poincaré Plots}

Figures 7.28 to 7.35 show examples of phase portraits of PAF episodes which belong to type $\mathrm{N}$ or $\mathrm{T}$ groups of the database previously defined in section 6.4 . This phase portraits are depicted for the spectral features $A_{1}, f_{p 1}, A_{2}$ and $f_{p 2}$. Figures 7.28 and 7.29 show the $A_{1}$ phase portraits of a type $\mathrm{N}$ episode and a type T episode, respectively. Similarly, Figures 7.30 and 7.31 compare the $f_{p 1}$ phase portraits of he same type $\mathrm{N}$ and $\mathrm{T}$ episodes. The pair of Figures 7.32 and 7.33 and the pair of figures 7.34 and 7.35 make the comparison of $A_{2}$ and $f_{p 2}$ phase portraits, respectively, of these two PAF episodes. Each pair of figures where equally scaled for every pair of figures of all spectral features in order to facilitate the comparison. $A_{1}(n-1), f_{p 1}(n-1), A_{2}(n-1)$ and $f_{p 2}(n-1)$ stand for the value in the previous observation moment to $A_{1}(n), f_{p 1}(n), A_{2}(n)$ and $f_{p 2}(n)$, respectively. Remember that a cubic spline is applied to the spectrograms so that a resolution of $0.01 \mathrm{~Hz}$ is accomplished.

The visual inspection of Poincaré plots of $f_{p 1}$ (Figures 7.30 and 7.31) show that both terminating and non-terminating episodes present lineal discontinuous clustering along the diagonal of the graph. Nonetheless, the dot clusters are located close to the bottom left-hand corner of the graph in the case of terminating episodes (type T). On the contrary, in the case of non-terminating episodes (type N), it can be observed that dot clusters are far from the the bottom left-hand corner. As shown in Figures 7.30 and 7.31, when a cursor is located at the place determined by the frequency of $5.5 \mathrm{~Hz}$ as a graphical threshold, the subdivision of the graph allowed us to distinguish between type $\mathrm{N}$ and type $\mathrm{T}$ episodes. The $85 \%$ of learning set recordings (17 out of 20 ) and the $70 \%$ of the test set recordings (21 out of 30) were correctly classified using this process. Tables 7.31 and 7.32 show the results of classification by $f_{p 1}$ Poincare plots for both learning and test sets, respectively.

In the rest of features it could not be found any plot characteristic useful to differentiate between type $\mathrm{N}$ and type $\mathrm{T}$ episodes. In consequence, no threshold could be fixed so that the percentage of correct classifications exceeded $50 \%$. Therefore, these last features were considered to be useless for the characterization of AF through the use of Poincaré plots. 


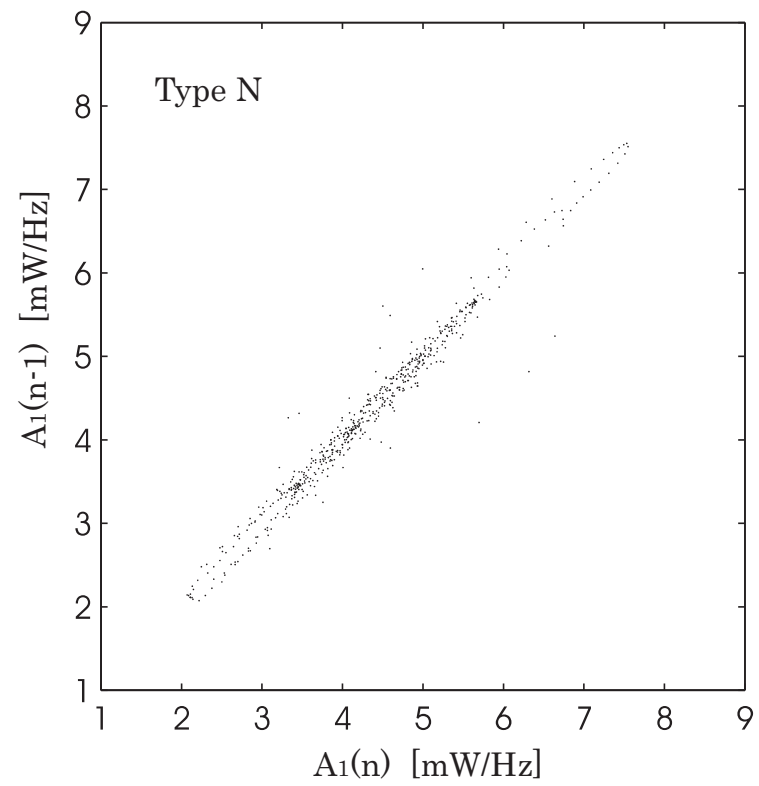

Figure 7.28. Example of $A_{1}$ phase portrait of a PAF episode belonging to type $\mathrm{N}$ group. No particular features of these plots can be attributed to the recording type.

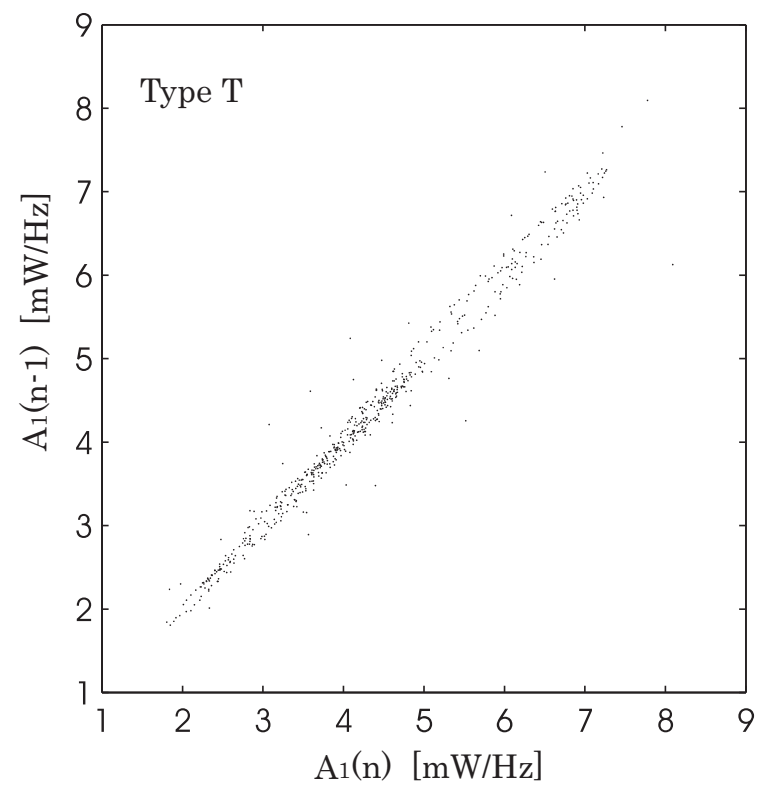

Figure 7.29. Example of $A_{1}$ phase portrait of a PAF episode belonging to type T group. No particular features of these plots can be attributed to the recording type. 


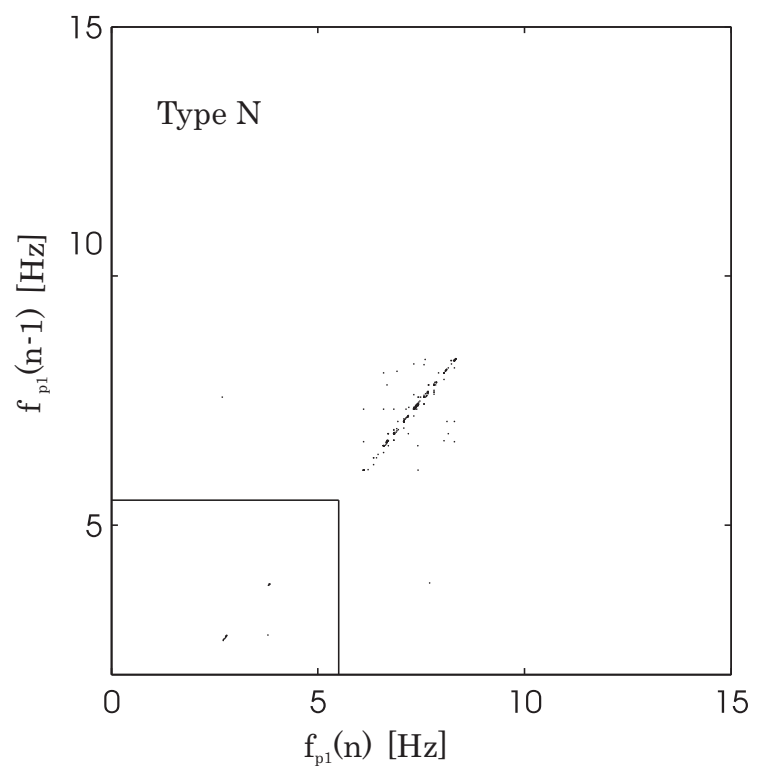

Figure 7.30. Example of $f_{p 1}$ phase portrait of a PAF episode belonging to type $\mathrm{N}$ group. Dot clusters are far from the bottom left-hand corner of the graph.

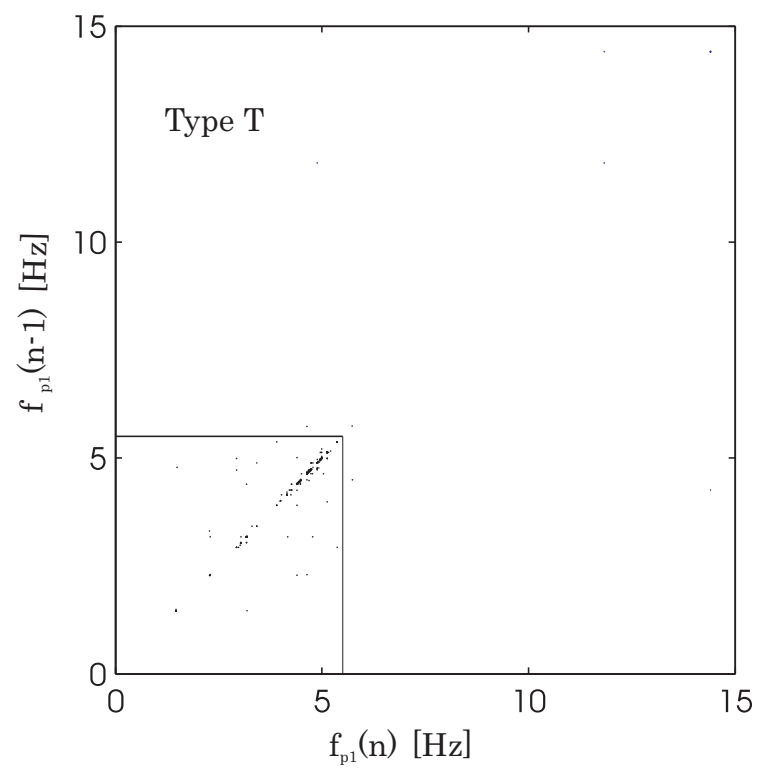

Figure 7.31. Example of $f_{p 1}$ phase portrait of a PAF episode belonging to type $\mathrm{T}$ group. Dot clusters are concentrated close to the bottom left-hand corner of the graph. 


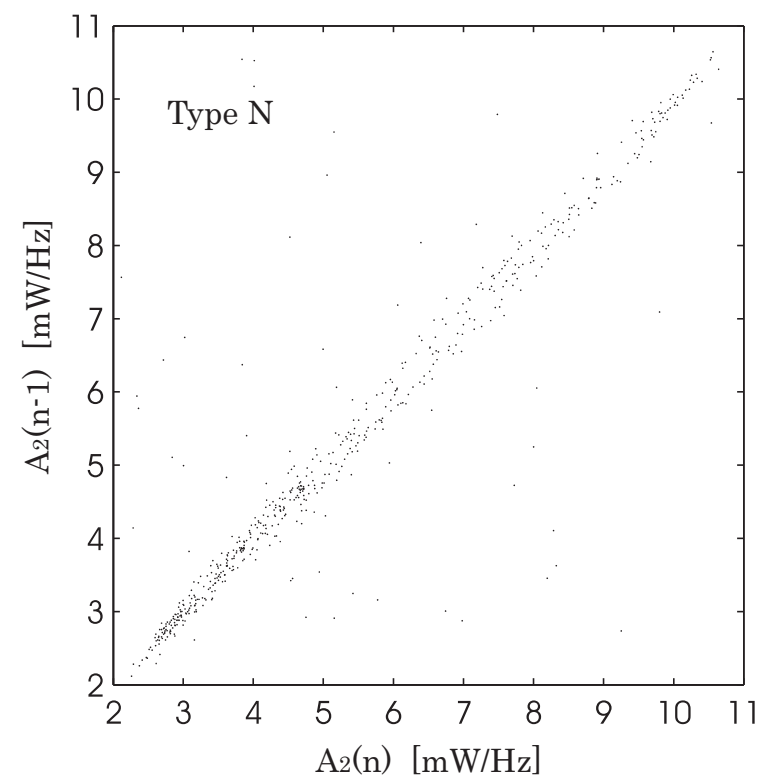

Figure 7.32. Example of $A_{2}$ phase portrait of a PAF episode belonging to type $\mathrm{N}$ group. No particular features of these plots can be attributed to the recording type.

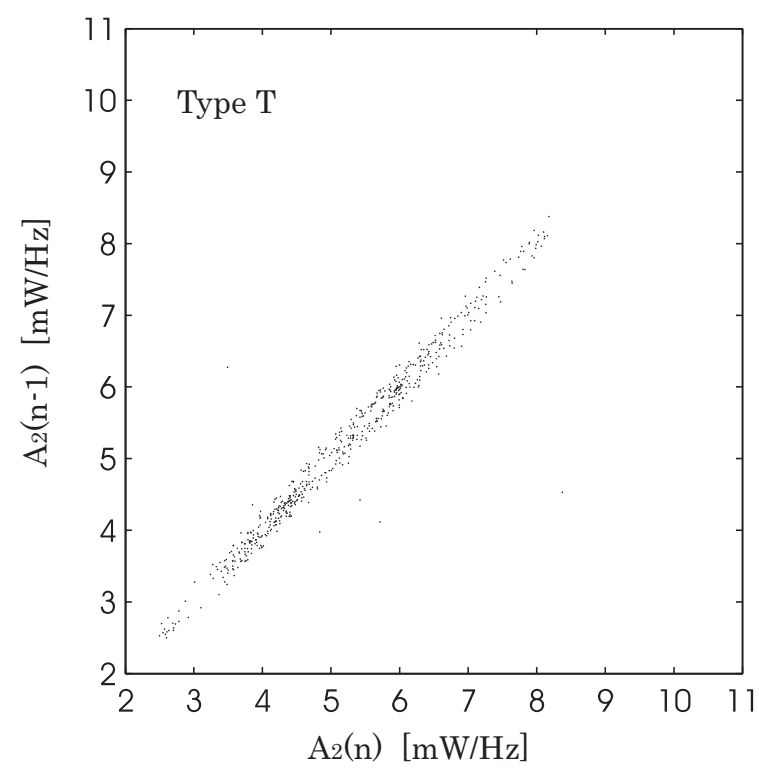

Figure 7.33. Example of $A_{2}$ phase portrait of a PAF episode belonging to $f$ the type $\mathrm{T}$ group. No particular features of these plots can be attributed to the recording type. 


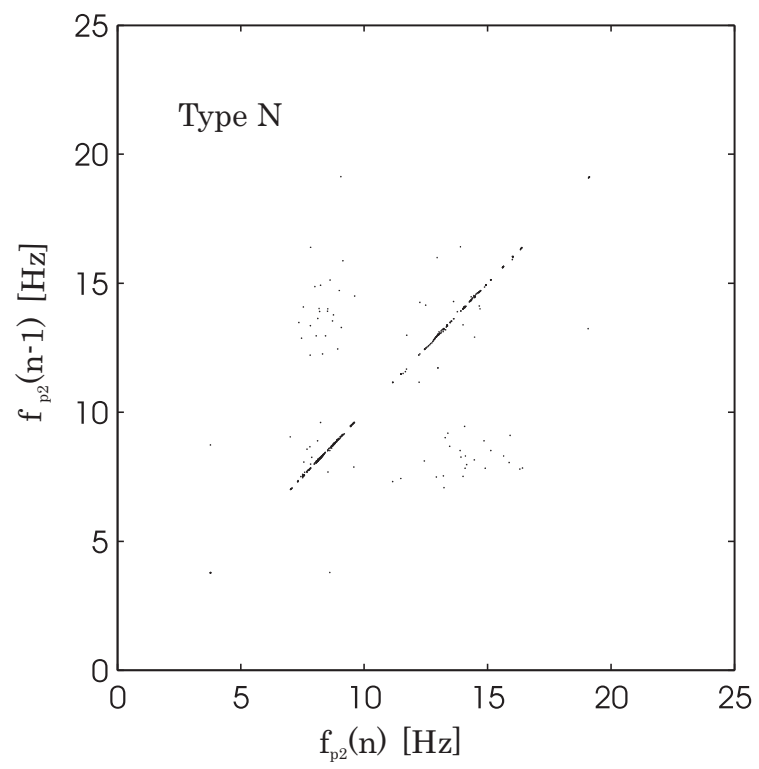

Figure 7.34. Example of $f_{p 2}$ phase portrait of a PAF episode belonging to type $\mathrm{N}$ group. No particular features of these plots can be attributed to the recording type.

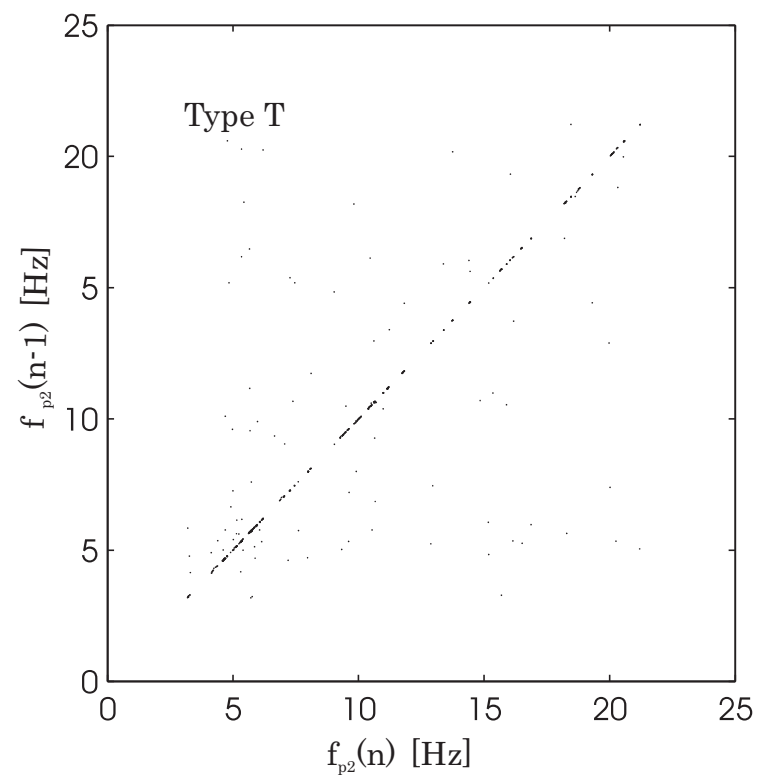

Figure 7.35. Example of $f_{p 2}$ phase portrait of a PAF episode belonging to type T group. No particular features of these plots can be attributed to the recording type. 


\begin{tabular}{c|c} 
Type N recordings & Type T recordings \\
\hline$a_{N} 1$ & $a_{T} 1$ \\
$a_{N} 2$ & $a_{T} 2$ \\
$a_{N} 3$ & $a_{T} 3$ \\
$a_{N} 4$ & $a_{T} 4$ \\
$a_{N} 5$ & $a_{T} 5$ \\
$a_{N} 6$ & $a_{T} 6$ \\
$a_{N} 7$ & $a_{T} 7$ \\
$a_{N} 8$ & $a_{T} 8$ \\
$a_{N} 9$ & $a_{T} 9$ \\
$a_{N} 10$ & $a_{T} 10$ \\
\hline
\end{tabular}

Table 7.31. Learning set recordings evaluated by Poincaré plots of $f_{p 1}$. Highlighted recordings were misclassified.

\begin{tabular}{cc|cc} 
Recording & Original Type & Recording & Original Type \\
\hline$b 1$ & $\mathrm{~N}$ & $b 16$ & $\mathrm{~N}$ \\
$b 2$ & $\mathrm{~T}$ & $b 17$ & $\mathrm{~N}$ \\
$b 3$ & $\mathrm{~T}$ & $b 18$ & $\mathrm{~T}$ \\
$b 4$ & $\mathrm{~N}$ & $b 19$ & $\mathrm{~T}$ \\
$b 5$ & $\mathrm{~N}$ & $b 20$ & $\mathrm{~N}$ \\
$b 6$ & $\mathrm{~T}$ & $b 21$ & $\mathrm{~N}$ \\
$b 7$ & $\mathrm{~N}$ & $b 22$ & $\mathrm{~N}$ \\
$b 8$ & $\mathrm{~N}$ & $b 23$ & $\mathrm{~T}$ \\
$b 9$ & $\mathrm{~T}$ & $b 24$ & $\mathrm{~T}$ \\
$b 10$ & $\mathrm{~N}$ & $b 25$ & $\mathrm{~T}$ \\
$b 11$ & $\mathrm{~T}$ & $b 26$ & $\mathrm{~N}$ \\
$b 12$ & $\mathrm{~T}$ & $b 27$ & $\mathrm{~T}$ \\
$b 13$ & $\mathrm{~N}$ & $b 28$ & $\mathrm{~N}$ \\
$b 14$ & $\mathrm{~T}$ & $b 29$ & $\mathrm{~T}$ \\
$b 15$ & $\mathrm{~N}$ & $b 30$ & $\mathrm{~N}$ \\
\hline
\end{tabular}

Table 7.32. Test set recordings evaluated by Poincaré plots of $f_{p 1}$. Highlighted recordings were misclassified. 


\title{
Chapter 8 \\ Discussion
}

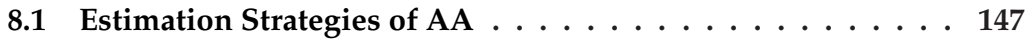 \\ 8.2 Prediction of PAF Termination $\ldots \ldots \ldots \ldots 151$
}

Two main subjects have guided the development of this thesis: the estimation strategies of AA, tackled in chapter 4, and the prediction of PAF termination, tackled in chapter 5. Therefore, the discussion is accordingly divided in two main sections. The discussion on estimation strategies is reported in section 8.1 and includes a analysis of results reached by CBSS algorithms and CMBS. The question of PAF termination prediction is raised in section 8.2, where results of MPSE are analyzed. This last section also comprises the topics of Poincaré plots and quantization effects.

\subsection{Estimation Strategies of AA}

The first environment for the study of CBSS algorithms, where the mixture process of AA and VA can be controlled, worked as a first approximation to the performance evaluation of these AA extraction methods. Infomax and TDD are the two CBSS algorithms that attained the best outcomes in the first environment in comparison with MBLMS and CoBliSS. The performance analysis of Infomax and TDD in terms of $R_{A A_{t}}$ - correlation between the original and the estimated AA in time domain - and $S I R_{A A}$ - improvement of the Signal to Interference Ratio of AA accomplished by BSS algorithms - indicate that both algorithms reach the best results when $N_{m}$, the length of mixing matrix filters, is near to one. That is, the highest quality of the extracted AA from synthetic AF ECGs is obtained when the original AA and VA are mixed according to an instantaneous model. Nonetheless, the analysis of results with regard to $N_{s}$, the length of the separation matrix filters, evidence the convolutive mixture of the original real signals used in the first environment. This fact is clearly highlighted when the $S I R_{A A}$ is 
regarded, since the maximum values of this index are not reached for $N_{s}$ equal to two, which is the closest approximation to the instantaneous separation model for Infomax and TDD. In contrast, the maximum mean values of $S I R_{A A}$ are reached for $N_{s}$ between 4 and 32 in the Infomax case, and for $N_{s}$ between 8 and 32 in the TDD case. A hidden convolutive mixture of the original sources could explain the better performance of Infomax and TDD at values of $N_{s}$ different from two. With respect to the comparison with FastICA, although Infomax and TDD can be considered as two good algorithms for AA extraction, results reveal that none of these CBSS algorithms is totally optimized for this purpose, in view that $R_{A A_{t}}$ and $S I R_{A A}$ values accomplished by FastICA are always higher.

The low values of $R_{A A_{t}}$ and $S I R_{A A}$ for MBLMS in the first environment makes clear that this CMBS algorithm is unsuitable for the AA extraction from AF ECGs. In the case of CoBliSS, the values of these indexes are not so low but are quite lower than those of Infomax and TDD. Hence, CoBliSS cannot be regarded as a good algorithm for AA extraction. In other words, neither MBLMS nor CoBliSS are able to achieve a sufficiently accurate AA to be considered in subsequent studies. Nevertheless, in spite of the low performance of these two CBSS algorithms, a slight performance improvement can still be observed in both algorithms when the convolutive model is used in the first environment to separate the AA from synthetic AF ECGs. As for Infomax and TDD, this effect was better perceived in the results when the attention is paid on the $R_{A A_{t}}$ index. The maximum values of this index were not reached for the closest approximation of these two CBSS algorithms to the instantaneous separation model, i.e. $N_{s}=2$ for TDD and $N_{s}=1$ for CoBliSS, which bears out a hidden convolutive mixture of the original AA and VA sources.

The joint description of the first environment results confirms the deductions extracted from the individual analysis of CBSS algorithms in this environment. Results corroborate that only two CBSS algorithms, namely Infomax and TDD, approximate the AA extraction performance of FastICA, attending to $R_{A A_{t}}$ and $S I R_{A A}$ indexes. The CBSS algorithm with the worst performance is MBLMS, and CoBliSS occupies an intermediate position with lower performance than TDD and Infomax. Some interesting cases of this joint description, as the maximum mean $S I R_{A A}$ of Infomax for $N_{s}=32$ or the maximum mean $S I R_{A A}$ of TDD for $N_{s}=8$, verify a main interpretation of the individual analysis, that is, the convolutive mixture of original sources.

The results of the second environment, where 12-leads ECGs of AF episodes are used, were only presented for the Infomax algorithm. There were two reasons for this. Firstly, the AA extraction performance reached by MBLMS and CoBliSS was so low that it was not worth analyzing these two CBSS algorithms in the second environment. Secondly, the TDD algorithm was not easily adaptable to the 12-lead ECGs of the second environment. One great advantage of this second environment is to be more realistic than the first and, consequently, its results are more functional for prospective clinical uses. On the other hand, one main difficult encountered in this second environment was to find a suitable AA signal as 
the original reference source for the computation of $R_{A A_{t}}$ and $S I R_{A A}$. The best available solution was that exposed in section 6.2 so that the AA is taken from the lead with the highest contribution of this source, usually lead V1. Given that this reference AA source is extracted by using ABS from a unique lead, its comparison with the AA extracted by CBSS algorithms could not to be totally appropriate. Since the AA extracted by CBSS algorithm draws together AA information from all leads, it would be more suitable to compare the estimated AA with a unified original AA, which is unavailable in the second environment. Despite this limitation, important remarks can be inferred from results of this environment. The comparison with FastICA shows that Infomax needs to be improved and adapted to the problem of AA extraction from real AF episodes. Nonetheless, although the $R_{A A_{t}}$ and the $S I R_{A A}$ of Infomax are several decibels lower than those of FastICA for any $N_{s}$ in the second environment, it can be appreciated a likely convolutive mixture of the cardioelectric sources that would explain the maximum of $S I R_{A A}$ with regard to $N_{s}=8$.

The lower AA extraction performance of CBSS algorithms in comparison with FastICA could have persuaded us to give up the study of these algorithms. Nonetheless, the performance results of CBSS algorithms also showed that the mixture of caridoelectric signals did not follow a totally instantaneous mixing model. This suggested the design of a new convolutive AA extraction algorithm that could improve the results of FastICA and, besides, be able to solve the lack of leads problem of Holter ECG recordings. This new algorithm, i.e. CMBS, is reasonably based on Infomax because of its higher AA extraction performance. The selection of TDD as a base for the CMBS design was also possible, given that a similar performance is obtained by this algorithm. However, the choice of TDD was early discarded in view of the problematic adaptation to 12-lead ECGs. Although CMBS has been applied in this thesis to 2-lead Holter ECGs, the number of leads of a Holter recording is usually three or even greater. Unlike TDD, Infomax allows that CMBS algorithm can be easily adapted to Holter recordings with a number of leads greater than two. Therefore, in order to exploit the information carried out by each Holter lead in future versions of CMBS, Infomax was the CBSS algorithm selected for the implementation of CMBS.

The performance of CMBS was analyzed in the two same environments for the other CBSS algorithms and was compared with the performance of FastICA and WBS. Both FastICA and WBS were built on the hypothesis of instantaneous mixture of sources. In short, CMBS can be viewed as an evolution of WBS where also the number of carioelectric sources is increased by using wavelet decomposition but the hypothesis of convolutive mixtures is used instead. The CMBS design and its performance analysis permitted us to know to what extent the AA extraction from Holter ECG recordings can be benefitted by considering the convolutive mixture model.

In the first environment, the performance comparison at three levels of SNR shows that FastICA is the worst affected algorithm by the AWGN. As a consequence of the least available observations, the performance of this algorithm is 
the worst affected by a contamination of signals with AWGN . On the other hand, results prove that CMBS is the robustest algorithm against AWGN. This effect is observed in all performance indicators, i.e. $R_{A A_{t}}, R_{A A_{f}}, f_{p 1}$, and $S C$. Therefore, it seems that the covolutive mixture model is best adapted to the AA extraction from Holter ECG and, besides, is better helped by an increase of observations via wavelet decomposition.

Results of $f_{p 1}$ - main frequency peak of AA - and $S C$ - spectral concentration of AA around $f_{p 1}-$ in the second environment confirm the superior robustness of CMBS against AWGN in comparison with FastICA and WBS. The convolutive mixture model seems to be better adapted to the AA extraction problem also in this scenario. Furthermore, FastICA is the worst affected algorithm by AWGN in this environment too, what bears out that the increase of observations from ECG recordings is a requirement to improve the accuracy of the extracted AA. On the other hand, the performance comparison of CMBS and ABS in this environment shows that similar estimations of $f_{p 1}$ are reached by both algorithms. This fact validates the results of CMBS in a scenario of real ECG recordings, attending to the wide acceptance of ABS as a time-domain technique for AA extraction. Besides, given that AWGN provokes a higher dispersion of estimated $f_{p 1}$ values in ABS, it can be considered that CMBS is robuster than ABS against noise. Since ABS works with a unique lead, the increase of observations explains why CMBS is stronger than ABS when sources are contaminated by AWGN. In sum, the higher robustness of CMBS against noise can be associated, in the one hand, to the upper diversity of observations and, in the other hand, to an enhancement of convolutive mixtures due to frequency band subdivision.

Our analysis shows up that CMBS improves the extraction performance of WBS and ICA in both scenarios so that a high accuracy of the estimated AA for synthetic and real AF ECG episodes is accomplished, what is proved by the high values of $R_{A A_{t}}$ - correlation between the original and the estimated AA in time domain. In addition, the high values of $R_{A A_{f}}$ - correlation between the original and the estimated AA in frequency domain - and $S C$ and the low error of $f_{p 1}$ estimation prove that the original spectral features of the AA are preserved in the AA estimated by CMBS from both synthetic and real signals. This fact enables CMBS as a suitable step previous to the analysis of AA signals in the time-frequency domain.

The main limitation of this study could be the reduced size of the recording database. In the first environment, this difficulty was overcame by using ten different mixing matrices for each filter length $N_{m}$. Consequently, 150 mixtures were analyzed in each experiment defined by $N_{m}$, attending to the fact that 15 pairs of AA and VA signals were available. This number of mixtures was deemed to be large enough for an accurate statistic analysis of results. In the second environment, the number of mixtures could not be augmented artificially, because this environment reproduces a working context of real ECG recordings. Therefore, only 20 mixtures could be analyzed for each separation matrix filter length $N_{s}$. In spite of this, important findings could be deduced from the analysis of CBSS 
algorithms in this context. At present, our research group is working on the construction of a new wider database of Holter ECG recordings from AF episodes in collaboration with the several hospitals.

\subsection{Prediction of PAF Termination}

In this thesis, we have introduced MPSE as a new method to predict the spontaneous termination of PAF episodes. This method is based on the spectral features regularity analysis of the AA extracted from Holter recordings of AF episodes. Accordingly to the best performance of CMBS for AA extraction from Holter ECG recordings, a subject discussed in the previous section, this algorithm was chosen as a previous step to PAF termination prediction. The $t$-test has revealed the existence of significant differences for six of the studied spectral features. Consequently, the selection of spectral features for the study, based on previous works on AF, can be considered appropriate. This test discloses that the mean SampEn of the relevant features is higher in the nonterminating than in the terminating $\mathrm{AF}$ episodes. Consequently, the nonterminating recordings seem to present more complex dynamics than the terminating ones. This corroborates the physiological organization increase of AA prior to AF termination, what was previously reported through invasive atrial electrograms [36, 147]. Furthermore, results indicate that clinical relevant information on AF organization can be obtained from the spectral features of the surface ECG.

Additionally, the $t$-test has revealed that the SampEn of $f_{p 1}$ has the highest predictive power among all the studied spectral features. In previous studies of AF termination $[22,24,25]$ the $f_{p 1}$ has been also revealed as a good predictor of AF termination. The main difference of our work with those studies is that we consider the mathematical regularity of spectral features in opposition to direct mean values.

The major relevancy of $S a m p E n$ for feature $f_{p 1}$ is confirmed by the discriminant analysis. The canonical coefficient for SampEn of $f_{p 1}(1.880)$ is the highest, thus it is the variable that contributes the most to the discriminant function. A slightly lower canonical coefficient is associated with the SampeEn of AA (1.662), what indicates that both variables, and consequently both time and frequency domains, have a similar weight in the prediction of $\mathrm{AF}$ termination. A smaller value of the canonical coefficients is related to the SampEn of $\Delta f_{p}(0.816)$. Nonetheless, we must not underestimate any of the variables. Since $\Delta f_{p}$ - normalized distance between the main and second spectral peaks of AA - is a description of the spectral shape, we can confirm that the variability of this shape along time contributes to the resultant discriminant function. On the other hand, all these three canonical coefficients have the same positive sign, what points up that the loss of mathematical regularity is an indicator of more likely maintenance of AF.

The discriminant analysis has provided an improvement of the results with 
respect to the classification by threshold (5\% for the learning set and $6.66 \%$ for the test set). For that reason, it is worth considering the discriminant analysis in predicting the evolution of AF because this improvement in the classification of AF could be of great importance in routine clinical practice. Finally, the crosscorrelation outcomes show that variables of the resultant discriminant function have cross-correlations between them that are not negligible. This means that these variables share information, that is, they are not totally independent. This would explain, to some extent, the high percentage of correctly classified episodes by the SampEn of $f_{p 1}$ and the additional percentage due to the discriminant analysis.

As MPSE is based on the mathematical regularity of spectral features, it is worth discussing now the most important observations on regularity. From results it can be firstly deduced that the future evolution of AF affects is not only reflected in the mean values of spectral features but also in their variability in time. Secondly, the SampEn of the spectral features is higher for the non-terminating than for the terminating episodes. Therefore, the spectral features mathematical regularity might be used to predict spontaneous paroxysmal AF termination and could provide information about the organization of atrial activation in AF. The good results make this new method a useful tool that can help clinicians in the management of AF. In comparison with previous classifiers of the Challenge, only the winner team [22] classified correctly more recordings than our method, one in the learning set. Nevertheless, the method presented in [22] was only based in the last second of the AF episode previous to AF termination, so that it cannot be easily extrapolated to databases different from the one of Challenge 2004. The results of the rest of classifiers have been enhanced by our method. Furthermore, MPSE is a new strategy that provides an assessment of spectral features regularity that is not present in previous works.

The study of the quantization effects on the accuracy of the extracted spectral features was developed in order to assess the minimum recommended number of encoding bits that allows a reliable analysis of AA signals by MPSE. This study revealed that eight bits can be considered as the minimum real resolution threshold than can provide acceptable results. However, this only can be affirmed when the effective dynamic range approximates to the nominal dynamic range. This would require a real-time adjustment of systems that is hardly available in Holter recorders. Therefore, taking into account the headroom margin that has to be considered to avoid recording overflow, the minimum recommended number of bits is twelve. Put differently, this study revealed the minimum criteria of Holter ECG recordings that are used as inputs of the MPSE algorithm. This will be mainly useful for establishing the desirable properties of the Holter ECG recordings that will constitute our database in construction. This implies a correct adjustment of the Holter ambulatory recorder, concretely a SEER Light Compact Digital recorder of GE Medical Systems.

The Poincaré plots are presented in this thesis as an additional method to MPSE for predicting the PAF termination by means of graphic analysis. The vi- 
sual interpretation of data and the low computational load are the main advantages of this method, which could be useful to the clinical practice. The results of this new method prove its suitability for PAF characterization when the spectral feature $f_{p 1}$ is used. Although the percentage of correctly classified PAF episodes is lower than the one reached by MPSE, this can be considered a useful method with acceptable results accordingly to the $85 \%$ of correct decisions for the test set and the $70 \%$ for the learning set. Further research based on Poincare plots and nonlinear time series analysis could improve present results. On the other hand, this lower percentage can be attributed to an intrinsic error of plot interpretation. Nevertheless, the Poincaré plots can be seen as a complementary way to examine the nonlinear spectral properties of AA signals. That is, the Poincare plots contribute a new perspective to the nonlinear analysis of AF ECGs. An in-depth analysis of Poincare plots with the aim to find a logical relation to MPSE results could through light on AA signal dynamics. Furthermore, this likely association between MPSE and Poincare plots could help to the improvement of the method, which is still in a initial development stage. While the method was thought for visual interpretation, an automatized examination of results would help to reduce lecture errors.

The prediction of PAF termination presents several limitations. Firstly, the database contains a reduced number of short recordings. A wider database would be advisable in order to validate the method's performance over a larger population. Longer recordings, which comprise AF onset and offset, would allow to study the AA organization evolution during the complete AF episode. As mentioned before, a wider database is being constructed in order to solve this problem. Secondly, no information about the medication of patients is provided in the database. Different drugs could have different effects on the registered wave form and consequently the results could be altered. The new database will be correctly noted and will include the necessary data to discriminate different clinical cases. Thirdly, although the Holter recordings have been oversampled to 1024 $\mathrm{Hz}$, we must take this with caution since this oversampling cannot be considered to be strictly the same as an original sampling rate of $1024 \mathrm{~Hz}$. The recordings of the new database will be all sampled at a original sample rate of $500 \mathrm{~Hz}$ so that the oversampling effect will be reduced to 2 . 



\section{Chapter 9 \\ Conclusions, Future Lines and Contributions}

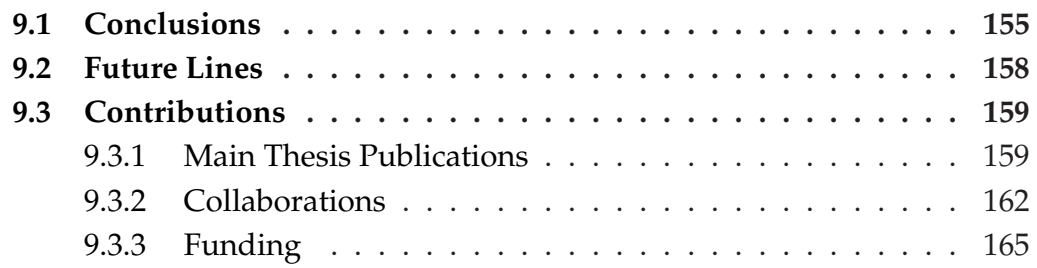

After the concise discussion on results of the previous chapter, here the main conclusions of the thesis are exposed in section 9.1. Given that the accomplishment of a research work always suggests undertaking additional tasks, likely future lines of development are mentioned in section 9.2. The main thesis contributions to scientific journals and the incentive research programs that partly funded the execution of this thesis are enumerated in section 9.3.

\subsection{Conclusions}

In chapter one, the four main objectives of this thesis were concisely exposed. The final conclusions are logically centered in the accomplishment of these initial objectives. With reference to the first objective, i.e. the assessment of the convolutive mixing model, several important conclusions can be extracted. Firstly, the performance analysis of the most relevant convolutive algorithms reveals that some of them approach the performance of the instantaneous algorithm for reference, i.e. FastICA. Nonetheless, convolutive algorithms need to be enhanced in order to reach the FastICA performance. Despite the convolutive model is a better description of the real mixture of cardioelectric sources, the AA extraction 
performance of instantaneous algorithms was not exceeded. These results are certainly surprising, and they can be explained by the fact that convolutive algorithms have been recently designed and need to be improved and adapted to the particular features of ECG signals. At first sight, solving the error provoked by the assumption of instantaneous source mixing in FastICA seems to be lower advantageous to CBSS algorithms accuracy than expected. This might be attributed to the low sampling rate of ECG recordings. Taking into account a maximum available sampling rates of $1 \mathrm{kHz}$, the time distance between ECG samples is $1 \mathrm{~ms}$, which is of the same order than the propagation error and the misalignment of QRS fiducial points. A sample rate around $10 \mathrm{kHz}$ or more would probably increase the performance because this would allow to better considerate both the propagation error and the QRS misalignment. Unfortunately, these sampling rates are not frequently available and would require much larger data storage capacities. Nevertheless, a second glance at results proved a certain convolutive behavior in the cardioelectric source mixture process. This permitted to progress in the study of CBSS algorithm and to design a new AA extraction method optimized for convolutive mixtures and Holter ECG recordings.

The second objective of this thesis is just related to the design of this new AA extraction algorithm, i.e. CBMS. The CMBS design was logically based on Infomax, given the good results of this algorithms in both first and second environments. The lack of leads problem for applying CBSS algorithms to the AA extraction from Holter ECG recordings was solved in CMBS by the band subdivision that the Wavelet transform figures out. One main decision of the CMBS design was the selection of the function used as a mother wavelet. The selected function was the symlet (8) with regard to its best results in comparison with other analyzed mother wavelets. Although the increase of leads by wavelet decomposition was firstly applied in [41], the utility of considering the convolutive model in this context of lack of leads is revealed as an important conclusion of the study. The increase of observations by wavelet transform produced an performance improvement of CMBS in comparison with WBS and FastICA and a higher robustness against noise. In other words, CMBS solves the lack of leads problem and, at the same time, its results prove the suitability of the convolutive mixing model for AA separation from Holter recordings. Furthermore, the comparison of CMBS with ABS confirms its appropriateness for AA extraction in noisy environments.

With reference to the third objective, that is, the assessment of the regularity estimator SampEn, several important conclusions could be deduced. Firstly, it was demonstrated that the $S a m p E n$ is able to find regularity differences in series of AA spectral features depending on the likeliness of a PAF episode to return to normal sinus rhythm. In other words, it was proved that SampEn can be used as an accurate estimator for predicting PAF termination by analyzing the spectral features regularity of the AA extracted from ECG recordings. Results showed that more regularity of spectral features series implies a higher likeliness of PAF episode termination. Conversely, lower regularity signifies a lower likeliness of the episode to evolve to normal sinus rhythm. Among the analyzed spectral fea- 
tures, $f_{p 1}$ was revealed as the most significant one, when the SampEn of series is computed, with a bilateral significance of 0.001 . Other relevant spectral features, namely $\Delta f_{p}, A_{1}, d_{1}$ and $S C$, were also revealed as significant for PAF termination prediction since the bilateral significance of their $S a m p E n$ is lower than 0.05 as well. This suggested the use of all these features in a multivariate analysis of the SampEn outcomes. Remember that $f_{p 1}$ is the main peak frequency of the AA, $A_{1}$ is the related magnitude of this peak, $d_{1}$ is the deviation of the main from its mean value, and $S C$ is the Spectral Concentration.

The systematic regularity estimation of spectral feature series for PAF termination prediction, when the AA is extracted from Holter ECG recordins by CMBS, constitutes the accomplishment of the last exposed objective, that is, the design of the MPSE algorithm. Since MPSE uses CMBS for AA extraction, one main advantage of this algorithm is that can be applied to Holter ECG recordings and that can consider a convolutive mixing model. The use of $f_{p 1}$ series as a unique input of MPSE or the use of several relevant spectral features defined two different versions of this new AA extraction method. The $t$-test applied to the SampEn of $f_{p 1}$ was referred to as the univariate version while the joint analysis of SampEn by considering several spectral features was referred to as the multivariate version. In the final design of the multivariate version, the joint application of SampEn to $f_{p 1}, \Delta f_{p}$ and $\mathrm{AA}$ in time domain was the best feature combination for PAF termination prediction. The AA in time domain was also included as a variable for analysis so that both time and frequency regularity features of signals could be taken into account. The rest of spectral features were not finally used as MPSE inputs since the high cross-correlations make them unuseful. As far as performance is concerned, the univariate analysis reaches good prediction results and has the advantage of being more easily applicable. On the other hand, the operation of the multivariate version is a little more complex but provides an increase of the correctly classified PAF episodes percentage. Although both versions of the algorithm can be considered suitable for PAF prediction, the improvement accomplished by the multivariate version might be crucial for a possible clinical use of this extraction method.

The visual analysis carried out in Poincaré plots, was used as an additional method for PAF termination prediction. This visual method offered a quite accurate prediction of PAF termination episodes, although does not reaches the performance of CMBS. Nevertheless, a further conclusion can be inferred from the Poincaré plots results: $f_{p 1}$ is revealed again as the most useful spectral feature for PAF characterization when used individually. Finally, the study of quantization effects served to make some advise on the minimum number of recommended bits for a correct use of MPSE. 


\subsection{Future Lines}

The work accomplished in this thesis is in line with previous and present studies of our research group. The AA extraction from ECG recordings by applying BSS was first introduced by Rieta in [10], where an instantaneous mixing model was considered. This thesis was initially conceived as a natural extension of [10] centered on the study of CBSS algorithms. The execution of the thesis has solved most of the initial questions and, at the same time, allows to discern new lines of future research. Some of these lines are enumerated next.

- An in-depth study of the sampling rate effects on the accuracy of CBSS algorithms. Higher sampling rates would allow a better consideration of propagation effects. A possible future work could consist of assessing the likely progressive improvement of AA extraction carried out by CBSS algorithms when increasing sampling rates are used. The accomplishment of this would depend on the availability of ECG recorders that work at high sampling rates.

- Exploration of the reasons that make the convolutive model robuster against noise than the instantaneous one for the AA extraction from Holter ECG recordings. This would allow to better understand the generation and mixing processes of cardioelectric sources in the human body.

- Implementation of a CMBS version for real-time operation. This would require to increase the execution speed and the total automatization of the process. Several criteria could be combined to select the correct AA among all separated sources, e.g. $S C$ and kurtosis of AA spectrum.

- Construction of a wider ECG database in order to make a more reliable evaluation of the current and new developed AA extraction techniques. Our research group is currently working on this new database in collaboration with several hospitals.

With respect to PAF termination prediction, this work is just a small part of a global line of our research group where other related works as [148] are included. Further future research as a continuation of this thesis work will be centered in the following items.

- Studying the organization evolution of AA spectral features during long term AF recordings. The study of AA regularity in the first minutes of PAF onset has been used in this thesis as a means of predicting PAF episodes evolution. An interesting future study could be the regularity analysis of spectral features along the whole PAF episode. This could be carried out by analyzing available Holter ECG recordings and could be helpful for taking appropriate decisions on PAF management. 
- A further interesting challenge could be to find a suitable relation between the SampEn of spectral features and the pathophysiological mechanisms of AF. Although the mathematical regularity of spectral features has served as a tool to discriminate between terminating and nonterminating PAF episodes, the physiological reasons that explain these differences on organization remain still unclear. The execution of this could help to disclose hidden processes of electric heart activation.

- Application of the methodologies presented here to other types of $\mathrm{AF}$, as persistent $\mathrm{AF}$, and the help to medical decisions, as whether to cardiovert the patient or not depending on the expected evolution of the arrhythmia. Moreover, the application of these new methodologies could be extended to other cardiac diseases different form AF.

- Exploration of alternative regularity indices, e.g. the Lempel-Ziv complexity or central tendency measure, to estimate the regularity of spectral features. This might contribute to reveal new aspects of spectral feature regularity useful for predicting the evolution of AF episodes.

- An in-depth study of the effects of drugs on the regularity of spectral features series and its relation with the likeliness of PAF episodes to terminate. This could help to the customized selection of drugs for each patient.

- As for CMBS, the real-time operation of MPSE and its assessment by using a wider database are also important matters of future development. As commented before, a database is currently being constructed in collaboration with the several hospitals.

\subsection{Contributions}

\subsubsection{Main Thesis Publications}

The work carried out in this thesis has been published in several scientific journals of international scope and has been presented in national and international conferences. Next these scientific contributions are enumerated.

The performance study of CBSS algorithms was published as an article of Lecture Notes in Computer Science:

- C Vayá, J J Rieta, C Sánchez, and D Moratal. Performance study of convolutive BSS algorithms applied to the ECG of atrial fibrillation. Lecture Notes in Computer Science 3889 (2006), 495-502.

and in the international journal IEEE Transactions on Biomedical Engineering: 
- C Vayá, J J Rieta, C Sánchez, and D Moratal. Convolutive blind source separation algorithms applied to the electrocardiogram of atrial fibrillation: Study of performance. IEEE Transactions on Biomedical Engineering 54, 8 (2007), 1530-1533.

Previous version of this part were contributed to the proceedings of national conferences in:

- C Vayá, J J Rieta, D Moratal, and C Sánchez. Rendimiento de los algoritmos BSS convolutivos en el estudio de la fibrilación auricular. In Simposium Nacional de la Unión Científica Internacional de Radio (2005), vol. 20, pages 1881-1884.

- C Vayá, J J Rieta, D Moratal, and C Sánchez. Estudio de la extracción de la fibrilación auricular mediante algoritmos BSS convolutivos. In Congreso Anual de la Sociedad Española de Ingeniería Biomédica (2005), vol. 23, pages 7-10.

and to the proceedings of an international conference in:

- C Vayá, J J Rieta, D Moratal, and C Sánchez. Feasibility and performance of methods based on statistical signal processing to study atrial fibrillation. In Conf Proc IEEE Computers in Cardiology (2005), vol. 32, pages 925-928

The initial versions of MPSE algorithm were presented in three international conferences:

- C Vayá, J J Rieta, R Alcaraz, C Sánchez, and R Cervigón. Prediction of atrial fibrillation termination by approximate entropy in the time-frequency domain. In Conf Proc IEEE Computers in Cardiology (2006), vol. 33, pages 589-592.

- C Vayá and J J Rieta. Analysis of spectrogram parameters organization applied to the characterization of atrial fibrillation. In Conf Proc IEEE Computers in Cardiology (2007), vol. 34, pages 509-512.

- C Vayá and J J Rieta. Combined analysis of time and frequency series regularity applied to the study of atrial fibrillation. In Conf Proc IEEE Computers in Cardiology (2008), vol. 35, pages 73-76.

and four national conferences:

- C Vayá, J J Rieta, R Alcaraz, J Mateo, and C Sánchez. Clasificación de la fibrilación auricular mediante análisis de complejidad en el dominio tiempofrecuencia. In Congreso Anual de la Sociedad Española de Ingeniería Biomédica (2006), vol. 24, pages 25-28. 
- C Vayá and J J Rieta. Caracterización de la fibrilación auricular mediante parametrización espectrográfica del electrocardiograma. In Congreso Anual de la Sociedad Española de Ingeniería Biomédica (2007), vol. 25, pages 214-217.

- C Vayá and J J Rieta. Análisis combinado de regularidad de series en tiempo y frecuencia aplicado al estudio de la fibrilación auricular. In Congreso Anual de la Sociedad Española de Ingeniería Biomédica, (2008), vol. 26, pages 182-185.

- C Vayá and J J Rieta. Predicción de terminación de la fibrilación auricular mediante medidas de regularidad de parámetros espectrales. In Simposium Nacional de la Unión Científica Internacional de Radio (2008), vol. 23, pages 49-52.

The final version was published in the journal Medical and Biological Engineering and Computing:

- C Vayá and J J Rieta. Time and frequency series combination for noninvasive regularity analysis of atrial fibrillation. Med Biol Eng Comput 47, 7 (2009), 687-696.

The method for PAF termination prediction based on Poincaré plots was presented as an international conference publication in:

- C Vayá, J J Rieta, J Mateo, and C Sánchez. Poincaré plots of time-frequency parameters applied to the prediction of atrial fibrillation termination. In Conf Proc IEEE Computers in Cardiology (2007), vol. 34, pages 569-572.

The effects of quantization on MPSE were contributed to the same conference in:

- C Vayá and J J Rieta. ECG signal quantization effects inthe analysis of atrial fibrillation. In Conf Proc IEEE Computers in Cardiology (2007), vol. 34, pages 477-480.

The CMBS algorithm was presented in two international conferences:

- C Vayá, J J Rieta, and R Alcaraz. Convolutive multiband blind separation to dissociate atrial from ventricular activity in atrial fibrillation. In Conf Proc IEEE Computers in Cardiology (2009), vol. 36, pages 713-716.

- C Vayá, and J J Rieta. Combination of covolutive blind signal separation and wavelet decomposition to extract the atrial activity in atrial fibrillation. In International Conference on Bio-inspired Systems and Signal Processing (2010), vol. 3, p. in press. 
and in a national conference:

- C Vayá, J J Rieta, and R Alcaraz. Extracción de la actividad auricular en fibrilación auricular mediante separación ciega multibanda de mezclas convolutivas. In Congreso Anual de la Sociedad Española de Ingeniería Biomédica (2009), vol. 27, pages 429-432.

The final version is currently under review to be published in the journal Biomedical Signal Processing and Control:

- C Vayá, and J J Rieta. Atrial Activity Extraction form Holter ECG by Convolutive Multiband Blind Separation in Atrial Fibrillation. Biomed Signal Process Control

\subsubsection{Collaborations}

Other contributions less related with the subjects of this thesis are the result of collaborating with other researchers on biomedical engineering. A main topic of collaboration was the study of ICA as a previous step to the analysis of CBSS algorithms. The applicability of ICA to the analysis of atrial tachyarrhythmias was studied in depth in a conference paper:

- J J Rieta, C Vayá, C Sánchez, F Castells, and J Millet. Aplicabilidad de la separación ciega de fuentes para el analisis de taquiarrtimias auriculares. In Congreso Anual de la Sociedad Española de Ingeniería Biomédica, volume 22, pages 391-394, 2004.

A derivation of atrial surface reentries in AF patients by surface ICA-estimated AA is presented as a journal article of Lecture Notes in Computer Science in:

- J J Rieta, F Hornero, C Sánchez, C Vayá, D Moratal, and J M Sanchis. Derivation of atrial surface reentries applying ICA to the standard ECG of patients in postoperative atrial fibrillation. Lecture Notes in Computer Science, 3889:478-485, 2006.

An assessment of epicardial atrial activation in atrial fibrillation by ICA was presented to two national conferences in:

- J J Rieta, C Sánchez, C Vayá, and D Moratal. Verificación no invasiva de la activación auricular epicárdica en episodios de fibrilación auricular. In Congreso Anual de la Sociedad Española de Ingeniería Biomédica, volume 23, pages 335-338, 2005. 
- J J Rieta, C Sánchez, C Vayá, D Moratal, and F Hornero. Estudio de reentradas en la superficie auricular mediante el electrocardiograma estándar en episodios de fibrilación auricular. In Simposium Nacional de la Unión Científica Internacional de Radio, volume 20, pages 1521-1524, 2005.

and as international contribution in:

- J J Rieta, C Hornero, C Sánchez, C Vayá, and D Moratal. Epicardial atrial activation assessment from the surface ECG in atrial fibrillation. In Conf Proc IEEE Computers in Cardiology, volume 32, pages 941-944, 2005.

The effects of filtering on the applicability of ICA to AA extraction is documented in:

- J J Rieta, F Hornero, C Sánchez, C Vayá, and D Moratal. Efecto del filtrado paso bajo en el análisis electrocardiográfico mediante técnicas basadas en ICA. In Congreso Anual de la Sociedad Española de Ingeniería Biomédica, volume 23, page 447.450, 2005.

Previously to the development of CMBS, the author of the thesis collaborated in other works concerned with the AA extraction by using the wavelet transform. These collaborations resulted in three presentations in national conferences:

- C Sánchez, J J Rieta, D Moratal, C Vayá, and J Millet. Comparación de técnicas wavelet para la extracción de actividad auricular en taquiarritmias supraventriculares. In Simposium Nacional de la Union Científica Internacional de Radio, volume 20, pages 409-411, 2005.

- C Sánchez, J J Rieta, D Moratal, C Vayá, J M Blas, and J Millet. Algortimo wavelet para la mejora del rendimiento en la extracción de actividad auricular mediante separación ciega de fuentes. In Congreso Anual de la Sociedad Española de Ingeniería Biomédica, volume 23, pages 109-112, 2005.

- C Sánchez, J J Rieta, D Moratal, C Vayá, J M Blas, and J Millet. Evaluación de técnicas wavelet para la extracción de actividad auricular en taquiarritmias supraventriculares. In Congreso Anual de la Sociedad Española de Ingeniería Biomédica, volume 23, pages 455-458, 2005.

in an international conference:

- D Sánchez, J J Rieta, C Vayá, R Cervigón, and J Millet. Atrial activity enhancement by blind source sequential separation. In Conf Proc IEEE Computers in Cardiology, volume 32, pages 937-940, 2005. 
and as a journal article of Lecture Notes in Computer Science:

- C Sánchez, J J Rieta, C Vayá, R Zangróniz, and J Millet. Wavelet denoising as preprocessing stage to improve ICA performance in atrial fibrillation analysis. Lecture Notes in Computer Science, 3889:486-494, 2006.

The author also collaborated in the prediction of PAF termination by means of series regularity predictors as contributions to national symposiums in:

- R Alcaraz, C Sánchez, C Vayá, R Cervigón, and J J Rieta. Aplicación de la entropía muestral en el dominio wavelet para predecir la terminación de arritmias cardíacas supraventriculares. In Simposium Nacional de la Unión Científica Internacional de Radio, volume 21, pages 1260-1263, 2006.

- R Alcaraz, J J Rieta, J Mateo, C Vayá, and C Sánchez. Anáalisis de reversión en episodios de fibrilación auricular mediante entropía muestral wavelet. In Congreso Anual de la Sociedad Española de Ingeniería Biomédica, volume 24, pages 339-342, 2006.

and and as contributions to an international conference in:

- R Alcaraz, C Vayá, Cervigón R, C Sánchez, and J J Rieta. Wavelet sample entropy: A new approach to predict termination of atrial fibrillation. In Conf Proc IEEE Computers in Cardiology, volume 33, pages 597-600, 2006.

An study of successful cardioversion in atrial fibrillation is treated in:

- R Cervigón, R Alcaraz, C Vayá, J Mateo, J Millet, and C Sánchez. Aplicación de la entropía muestral para la prediccion de recurrencias en episodios de fibrilación auricular. In Congreso Anual de la Sociedad Española de Ingeniería Biomédica, volume 24, pages 451-454, 2006.

- R Cervigón, R Alcaraz, C Vayá, J Mateo, J Millet, and C Sánchez. Prediction of successful cardioversion in atrial fibrillation using wavelet analysis parameters and sample entropy. In Conf Proc IEEE Computers in Cardiology, volume 33, pages 593-596, 2006.

Finally, a collaboration on ECG signal denoising resulted in a national conference presentation:

- J Mateo, C Sánchez, R Alcaraz, C Vayá, and J J Rieta. Métodos de preprocesado en análisis electrocardiográfico: Problemática y evaluación de soluciones. In Congreso Anual de la Sociedad Española de Ingeniería Biomédica, volume 24, pages 543-546, 2006. 
two contributions to international conferences:

- J Mateo, C Sánchez, C Vayá, R Cervigón, and J J Rieta. A new adaptive approach to remove baseline wander from ECG recordings using madeline structure. In Conf Proc IEEE Computers in Cardiology, volume 34, pages 533536, 2007.

- J Mateo, C Sánchez, R Alcaraz, C Vayá, and J J Rieta. Neural networks based approach to remove baseline drift in biomedical signals. In 11th Mediterranean Conference on Medical and Biological Engineering and Computing, volume 16, pages 90-93, 2007.

and an international journal article:

- J Mateo, Sánchez C, C Vayá, R Cervigón, and J J Rieta. A learning based Widrow-Hoff delta algorithm for noise eduction in biomedical signals. Lecture Notes in Computer Science, 4527:377-386, 2007.

\subsubsection{Funding}

This work was partly funded by the following research incentive programs:

- From 01/01/2004 to 01/01/2006. "Clasificación y caracterización de taquiarritmias auriculares mediante distribuciones tiempo-frecuencia". Consellería de Empresa, Universidad y Ciencia de la Generalitat Valenciana. Amount: $26.400 €$.

- From 01/12/2006 to 01/12/2007. "Estudio de las contracciones normales y prematuras para la prevención de arritmias supraventriculares postoperatorias de cirugía cardíaca". Consellería de Empresa, Universidad y Ciencia de la Generalitat Valenciana. Amount: $13.200 €$.

- From 12/31/2006 to 12/31/ 2007. "Aplicación del Procesado Avanzado de Señal en la Extracción, Caracterización y Predicción de la Fibrilación Auricular Postoperatoria". Vicerrectorado de I+D+i de la Universidad Politécnica de Valencia (UPV20070086). Amount: $3.000 €$.

- From 10/01/2007 to 10/01/2010. "Sinergia de Metodos Lineales y No Lineales para el Analisis, Estratificacion y Prediccion del Comportamiento de la Fibrilacion Auricular". Ministerio de Educación y Ciencia -D.G. Investigacion (TEC2007-64884). Amount: 79.013€.

- From 09/16/2008 to 09/16/2009. "Ayuda cofinanciación UPV: Sinergia de Metodos Lineales y No Lineales para el Analisis, Estratificacion y Prediccion del Comportamiento de la Fibrilacion Auricular". Universidad Politécnica de Valencia (PAID-05-08). Amount: $3.500 €$. 



\section{Bibliography}

[1] V Fuster, L E Ryden, R W Asinger, and et al. CC/AHA/ESC guidelines for the management of patients with atrial fibrillation. Journal of the American College of Cardiology, 38(4):1266/I-1266/LXX, 2001.

[2] M Allessie, K Konings, and M Wijffels. Atrial Arrhytmias - State of the Art: Electrophysiological Mechanism of Atrial Fibrillation. J.P. DiMarco and E. N. Prystowsky, Eds. Armonk NY: Futura, 1995. ISBN 0-879-936-04-5.

[3] P A Wolf, R D Abbott, and W B Kannel. Atrial fibrillation as an independent risk factor for stroke: the Framingham Study. Stroke, 22:983-988, 1991.

[4] P Dorian, W Jung, and et al. The impairment of health-related quality of life in patients with intermittent atrial fibrillation: implications for the assessment of investigational therapy. J Am Coll Cardiol, 36:1303-1309, 2000.

[5] F Pizzetti, F M Turazza, M F Franzosi, and et al. Incidence and prognostic significance of atrial fibrillation in acute myocardial infarction: the GISSI-3 data. Heart, 86:527-532, 2001.

[6] K Wachtell, M Lehto, E Gerdts, and et al. Angiotensin II receptor blockade reduces new-onset atrial fibrillation and subsequent stroke compared to atenolol. The Losartan Intervention for End Point Reduction in Hypertension (LIFE) Study. J Am Coll Cardiol, 45:712-719, 2005.

[7] T J Wang, M G Larson, D Levy, and et al. Temporal relations of atrial fibrillation and congestive heart failure and their joint influence on mortality. The Framingham Heart Study. Circulation, 107:2920-2925, 2003.

[8] V Fuster, L E Rydén, D S Cannom, and et al. CC/AHA/ESC guidelines for the management of patients with atrial fibrillation-executive summary. European Heart Journal, 27:1979-2030, 2006.

[9] P Langley, J J Rieta, M Stridh, J Millet, L Sörnmo, and A Murray. Comparison of atrial signal extraction algorithms in 12-lead ECGs with atrial fibrillation. IEEE Transactions on Biomedical Engineering, 53(2):343-346, 2006. 
[10] J J Rieta. Atrial activity estimation in atrial fibrillation episodes by means of Blind Source Separation. PhD thesis, Universidad Politécnica de Valencia, Valencia, Spain, 2003.

[11] J J Rieta, F Castells, C Sánchez, and V Zarzoso. Atrial activity extraction for atrial fibrillation analysis using blind source separation. IEEE Transactions on Biomedical Engineering, 51(7):1176-1186, 2004.

[12] J Slocum, A Sahakian, and S Swiryn. Diagnosis of atrial fibrillation from surface electrocardiogram based on computer-detected atrial activity. Journal of Electrocardiology, 25(1):1-8, 1992.

[13] J F Cardoso. Blind signal separation: Statistical principles. Proceedings of the IEEE, 9(10):2009-2025, 1998.

[14] A Hyvärinen, J Karhunen, and E Oja. Independent Component Analysis. John Wiley \& Sons, New York, 2001.

[15] V Zarzoso and A K Nandi. Blind source separation. In A K Nandi, editor, Blind Estimation Using Higher-Order Statistics, pages 167-252. Boston, MA: Kluwer, 1999.

[16] P Comon. Independent components analysis - A new concept? Signal Processing, 36(3):287-314, 1994.

[17] R Plonsey and D B Heppner. Considerations of quasi-stationarity in electrophysiological systems. Bulletin of Mathematical Biophysics, 29(4):657-664, 1967.

[18] C R Kerr, K H Humphries, and et al. Progression to chronic atrial fibrillation after the initial diagnosis of paroxysmal atrial fibrillation: Results from the canadian registry of atrial fibrillation. American Heart Journal, 149(3):489496, 2005.

[19] S M Al-Khatib and et al. Observations on the transition from intermittent to permanent atrial fibrillation. Am Heart J, 140(1):142-145, 2000.

[20] C Blomström-Lundqvist, M M Scheinman, E M Aliot, J S Alpert, and et al. ACC/AHA/ESC guidelines for the management of patients with supraventricular arrhythmias-executive summary. Circulation, 108(15):1871-1909, 2003.

[21] G B Moody. Spontaneous termination of atrial fibrillation: A challenge from PhysioNet and Computers in Cardiology 2004. In Proc.Int.Conf on Computers in Cardiology, pages 101-104, 2004.

[22] S Petrutiu, A V Sahakian, and J Ng Swiryn. Analysis of the surface electrocardiogram to predict termination of atrial fibrillation: The 2004 Computers in Cardiology/PhysioNet Challenge. In Proc.Int.Conf on Computers in Cardiology, pages 105-108, 2004. 
[23] C Mora, F Castells, R Ruiz, J J Rieta, J Millet, and C Sánchez. Prediction of spontaneous termination of atrial fibrillation using time frequency analysis of the atrial fibrillatory wave. In Proc.Int.Conf on Computers in Cardiology, pages 109-112, 2004.

[24] D Hayn, K Edegger, P Scherr, and P Lercher. Automated prediction of spontaneous termination of atrial fibrillation from electrocardiograms. In Proc.Int.Conf on Computers in Cardiology, pages 117-120, 2004.

[25] F Cantini, F Conforti, M Varanini, F Chiarugi, and G Vrouchos. Predicting the end of an atrial fibrillation episode: The physionet challenge. In Proc.Int.Conf on Computers in Cardiology, pages 121-124, 2004.

[26] F Nilsson, M Stridh, A Bollman, and L Sörnmo. Predicting spontaneous termination of atrial fibrillation with time-frequency information. In Proc.Int.Conf on Computers in Cardiology, pages 657-660, 2004.

[27] M Lemay, Z Ihara, J M Vesin, and L Kappenberger. Computers in cardiology/physionet challenge 2004 : AF classification based on clinical features. In Proc.Int.Conf on Computers in Cardiology, pages 669-672, 2004.

[28] F Chiarugi, M Varanini, F Cantini, F Conforti, and G Vrouchos. Noninvasive ECG as a tool for predicting termination of paroxysmal atrial fibrillation. IEEE Transactions on Biomedical Engineering, 54(8):1399-1406, 2007.

[29] R Alcaraz and J J Rieta. Sample entropy of the main atrial wave predicts spontaneous termination of paroxysmal atrial fibrillation. Medical engineering and physics, 31(8):917-922, 2009.

[30] D Husser, M Stridh, L Sörnmo, I Toepffer, H U Klein, S B Olsson, and A Bollmann. Electroatriography - time-frequency analysis of atrial fibrillation from modified 12-lead ECG configurations for improved diagnosis and therapy. Medical Hypotheses, 68(6):568-573, 2007.

[31] A Bollmann, D Husser, L Mainardi, F Lombardi, P Langley, A Murray, J J Rieta, J Millet, S B Olsson, M Stridh, and Sornmo L. Analysis of surface electrocardiograms in atrial fibrillation: Techniques, research and clinical applications. Europace, 8(11):911-926, 2006.

[32] R Plonsey and R C Barr. Bioelectricity: a quantitative approach. Third Ed. Kluwer Academic Pub, 2007.

[33] S Petrutiu, N G Jason, and et al. Atrial fibrillation and waveform characterization. A time domain perspective in the surface ECG. IEEE engineering in medicine and biology, 25(6):24-30, 2006.

[34] F Ravelli, M Masè, M D Greco, L Faes, and M Disertori. Deterioration of organization in the first minutes of atrial fibrillation: A beat-to-beat analysis of cycle length and wave similarity. J Cardiovasc Electrophysiol, 18(1):60-65, 2007. 
[35] R Alcaraz and J J Rieta. Non-invasive organization variation assessment in the onset and termination of paroxysmal atrial fibrillation. Comput Methods Programs Biomed, 93(2):148-154, 2009.

[36] J Kneller and et al. Mechanisms of atrial fibrillation termination by pure sodium channel blockade in an ionically-realistic mathematical model. Cir Res, 96(5):e35-e47, 2005.

[37] M Holm, S Pehrson, M Ingemansson, L Sörmno, R Jahansson, L Sandhall, M Sunemark, B Smideberg, C Olsson, and S B Olsson. Non-invasive assessment of the atrial cycle length during atrial fibrillation in man : Introducing, validating and illustrating a new ECG method. Cardiovascular Research, 38:69-81, 1998.

[38] J G Akar, T H Everett, L C Kok, J R Moorman, and D E Haines. Effect of electrical and structural remodeling on spatiotemporal organization in acute and persistent atrial fibrillation. Cardiovascular Electrophysiology, 13(10):1027-1034, 2002.

[39] Y Takashaky, P Sanders, P Jaïs, and et al. Organization of frequency spectra of atrial fibrillation: Relevance to radiofrequency catheter ablation. $J$ Cardiovasc Electrophysiol, 17(4):382-388, 2006.

[40] P Sanders, O Berenfeld, M Hocini, P Jaïs, R Vaidyanathan, L-F Hsu, S Garrigue, Y Takahashi, M Rotter, F Sacher, C Scavée, R Ploutz-Snyder, J Jalife, and M Haïssaguerre. Spectral analysis identifies sites of high-frequency activity maintaining atrial fibrillation in humans. Circulation, 112(6):789-797, 2005.

[41] C Sánchez, J J Rieta, F Castells, R Alcaraz, and J Millet. Wavelet blind separation: A new methodology for the analysis of atrial fibrillation from holter recordings. In Conf Proc IEEE Computers in Cardiology, volume 31, pages 417-420, 2004.

[42] S A Chen and C T Tai. Is analysis of fibrillatory waves useful for treatment of atrial fibrillation? J Cardiovasc Electrophysiol, 15(8):918-919, 2004.

[43] J S Richman and J R Moorman. Physiological time-series analysis using approximate entropy and sample entropy. Am J Physiol Heart Circ Physiol, 278(6):H2039-H2049, 2000.

[44] A Bollmann. Quantification of electrical remodeling in human atrial fibrillation. Cardiovascular Research, 47(2):207-209, 2000.

[45] A Goette, C Honeycutt, and J J Langberg. Electrical remodeling in atrial fibrillation. Time course and mechanisms. Circulation, 94(11):2968-2974, 1996.

[46] R Hornero, D Abasolo, N Jimeno, Sanchez C I, J Poza, and M Aboy. Variability, regularity and complexity of time series generated by schizophrenic patients and control subjects. IEEE Transactions on Biomedical Engineering, 53(2):210-218, 2006. 
[47] Jiun Tuan, Faizel Osman, Mohamed Jeilan, Suman Kundu, Rajkumar Mantravadi, Peter J. Stafford, and G. AndrÃ (c) Ng. Increase in organization index predicts atrial fibrillation termination with flecainide post-ablation: spectral analysis of intracardiac electrograms. Europace, 2009.

[48] C Starr and B McMillan. Human Biology. Thomson Learning, 2002.

[49] R E Klanbunde. Cardiovascular Physiology Concepts. Lippincott Williams and Wilkins, 2004.

[50] J Malmivuo and R Plonsey. Bioelectromagnetism: Principles and Applications of Bioelectric and Biomagnetic Fields. Oxford University Press, 1995.

[51] J M Ferrero, J Saiz, and A Arnau. Bioelectrónica. Señales bioelectrónicas. Servicio de Publicaciones UPV, 1994.

[52] R Paine. Generation and interpretation of the electrocardiogram. Lea \& Febiger, Philadelphia, 1988.

[53] S Díaz, J M Lobos, P Conthe, J Ortigosa, and L Silva. Electrocardiografía. Biblioteca multimedia semFYC, Madrid, 2003.

[54] R M Gulrajani. The forward and the inverse problem of electrocardiography. IEEE Engineering in Medicine and Biology Magazine, 17(5):84-101, 1998.

[55] D Kilpatric and P Johnston. Origin of the electrocardiogram. IEEE Engineering in Medicine and Biology Magazine, 13(4):479-486, 1994.

[56] D B Geselowitz. On the theory of the electrocardiogram. Proceedings of the IEEE, 77(6):857-876, 1989.

[57] M Wijffels, C Kirchhof, R Dorland, and M Allessie. Atrial fibrillation begets atrial fibrillation. A study in awake chronically instrumented goats. Circulation, 92(7):1954-1968, 1995.

[58] G D Veenhuyzen, C S Simpson, and H Abdollah. Atrial fibrillation. Can Med Assoc, 171:755-760, 2004.

[59] E Leistad, G Aksnes, E Verburg, and G Christensen. Atrial contractile dysfunction after short-term atrial fibrillation is reduced by verapamil but increased by BAY K86. Circulation, 93(9):1747-1754, 1996.

[60] E G Daoud, P Marcovitz, B P Knight, and et al. Short-term effect of atrial fibrillation on atrial contractile function in humans. Circulation, 99(23):30243027, 1999.

[61] D S G Conway and G Y H Lip. Cardiac Arrhythmias, chapter Atrial physiology. Mosby Publishing, 2003.

[62] D V Unverferth, R H Fertel, and et al. Atrial fibrillation in mitral stenosis: histologic, hemodynamic and metabolic factors. Int J Cardiol, 5(2):143-154, 1984. 
[63] B Burstein, B Sc Stanley, and M D Nattel. Atrial fibrosis: Mechanisms and clinical relevance in atrial fibrillation. Journal of the American College of Cardiology, 51(8):802-809, 2008.

[64] S Rush and H Larsen. A practical algorithm for solving dynamic membrane equations. IEEE Trans Biomed Eng, 25(4):389-392, 1978.

[65] A Bollmann, D Husser, M Stridh, L Sörnmo, M Majic, H U Klein, and S B Olsson. Frequency measures obtained from the surface ECG in atrial fibrillation research and clinical decisssion-making. Cardiovascular Electrophysiology, 14:S154-S161, 2003.

[66] G K Moe. On multiple wavelet hypothesis of atrial fibrillation. Archives Internationales de Pharmacodynamie et de Therapiel, 140:1-2, 1964.

[67] Z Qu and J N Weiss. Dynamics and cardiac arrhythmias. J Cardiovasc Electrophysiol, 17(9):1042-1049, 2006.

[68] A Bollmann and F Lombardi. Electrocardiology of atrial fibrillation. Current knowledge and future challenges. IEEE engineering in medicine and biology, 6(25):15-23, 2006.

[69] A S Go, E M Hylek, K A Philips, and et al. Prevalence of diagnosed atrial fibrillation in adults: national implications for rhythm management and stroke prevention: the AnTicoagulation and Risk Factors in Atrial Fibrillation (ATRIA) Study. JAMA, 285(18):2370-2375, 2001.

[70] E D Benjamin, D Levy, and et al. Independent risk factors for atrial fibrillation in a population-based cohort. JAMA, 271:840-844, 1994.

[71] D A Dulli, H Stanko, and R L Levine. Atrial fibrillation is associated with severe acute ischemic stroke. Neuroepidemiology, 22:118-123, 2003.

[72] E J Benjamin, P A Wolf, and et al. Impact of atrial fibrillation on the risk of death: the Framingham Heart Study. Circulation, 98:946-952, 1998.

[73] R C Barr, T C Pikington, J P Boineau, and M S Spach. Determining surface potentials from current dipoles, with application to electrocardiography. IEEE Transactions on Biomedical Engineering, 13:88-92, 1966.

[74] J J Rieta, F Castells, C Sánchez, D Moratal-Pérez, and J Millet. Bioelectric model of atrial fibrillation: applicability of blind source separation techniques for atrial activity estimation in atrial fibrillation episodes. In Conf Proc IEEE Computers in Cardiology, volume 30, pages 525-528, 2003.

[75] R C Barr, M Ramsey, and M S Spach. Relating epicardial to body surface potential distribution by means of transfer coefficients based on geometry measurements. IEEE Transactions on Biomedical Engineering, 24(1):1-11, 1977. 
[76] R N Klepfer, C R Johnson, and R S MacLeod. The effects of inhomogeneities and anisotropies on electrocardiographic fields: A 3-d finite-element study. IEEE Transactions on Biomedical Engineering, 44(8):706-719, 1997.

[77] S Levy, G Breithardt, R W F Campbell, A J Camm, and et al. Atrial fibrillation: current knowledge and recommendations for management. European Heart Journal, 19(9):1294-1320, 1998.

[78] M Stridh and L Sörnmo. Spatiotemporal QRST cancellation techniques for analysis of atrial fibrillation. IEEE Transactions on Biomedical Engineering, 48(1):19-27, 2001.

[79] S Shkurovich, A Sahakian, and S Wirkyn. Detection of atrial activity from high-voltage leads of implantable ventricular defibrillators using a cancellation technique. IEEE Transactions on Biomedical Engineering, 45:229-234, 1998.

[80] A Bollmann, K Kanuru, K K McTeague, and et al. Frequency analysis of human atrial fibrillation using the surface electrocardiogram and its response to ibutilide. American Journal of Cardiology, 81(12):1439-1445, 1998.

[81] N V Thakor and Y S Zhu. Applications of adaptive filtering to ECG analysis: Noise cancellation and arrhytmia detection. IEEE Transactions on Biomedical Engineering, 38(8):785-794, 1991.

[82] W.J. Tomkins. Biomedical digital signal processing: C language examples and laboratory experiments for the IBM PC. Prentice Hall, 1993. ISBN 0-13-0672165.

[83] W A H Engelse and C Zeelemberg. A single scan algorithm for QRSdetection and feature extraction. In Proc.Int.Conf on Computers in Cardiology, volume 6, pages 37-42, 1979.

[84] J Slocum. Use of the autocorrelation function to detect atrial fibrilllatory activity on the surface electrocardiogram. In Proceedings of the Annual International Conference of the IEEE Engineering in Medicine and Biology Society, volume 13, pages 732-733, 1991.

[85] J Slocum and P Wang. Preliminary study on the discrimination of atrial fibrillation and regular tachycardias using a measure of non-uniformity in intra-atrial leads. In Conf Proc IEEE Computers in Cardiology, volume 22, pages 231-233, 1992.

[86] R Alcaraz and J J Rieta. Optimal beat selection study for QSRT cancellation methods in the ECG of atrial fibrillation. In Proc Int Conf on Computers in Cardiology, pages 1045-1048, 2008.

[87] R Alcaraz and J J Rieta. Adaptive singular value cancellation of ventricular activity in single-lead atrial fibrillation electrocardiograms. Physiological Measurement, 29(12):1351-1369, 2008. 
[88] Q Xi, A V Sahakian, and S Swiryn. The effect of QRS cancellation on atrial fibrillatory wave signal characteristics in the surface ECG. J Electrocardiol, 36(3):243-249, 2003.

[89] F Castells, C Mora, J J Rieta, D Moratal-Pérez, and J Millet. Estimation of atrial fibrillatory wave from single-lead atrial fibrillation electrocardiograms using principal component analysis concepts. Med Biol Eng Comput, 43(5):557-560, 2005.

[90] M Lemay, J M Vesin, A Oosterom, V Jacquemet, and L Kappenberger. Cancellation of ventricular activity $n$ the ECG: evaluation of novel and existing methods. EEE Trans Biomed Eng, 54(5):542-546, 2007.

[91] A Bollmann. Quantification of electrical remodeling in human atrial fibrillation. Cardiovasc Res, 47(2):207-209, 2000.

[92] J J Rieta, F Castells, C Sánchez, and J Igual. ICA applied to atrial fibrillation analysis. In International Symposium on Independent Component Analysis and Blind Signal Separation, pages 59-64, 2003.

[93] F Castells, J J Rieta, J Millet, and V Zarzoso. Spatiotemporal blind source separation approach to atrial activity estimation in atrial tachyarrhythmias. IEEE Transactions on Biomedical Engineering, 52(2):258-267, 2005.

[94] J A Gubner. Probability and Random Processes for Electrical and Computer Engineers. Cambridge University Press, 2006.

[95] S A Cruces and L Castedo. Stability analysis of adaptive algorithms for blind source separation of convolutive mixtures. Signal Processing: the Official Publication of the European Association for Signal Processing (Eurasip), 78(3):265-275, 1999.

[96] A Cruces and A Cichocki. Combining blind source extraction with joint approximate diagonalization: Thin algorithms for ICA. In International Symposium on Independent Component Analysis and Blind Signal Separation, volume 4, pages 463-468, 2003.

[97] R H Lambert. Multichanel Blind Deconvolution: FIR matrix algebra and separation of multipath mixtures. PH.D, University of Southern California, 1996.

[98] J M Sanchis. Evaluación de las condiciones del sistema de mezcla en la separación ciega de fuentes de mezcla convolutiva para aplicaciones de audio. PhD thesis, Universidad Politécnica de Valencia, Valencia, Spain, 2003.

[99] R W Liu and Y Inouye. Direct blind signal separation of convolutive mixtures of white spatially independent non-gaussian signals. In International Symposium on Independent Component Analysis and Blind Signal Separation, pages $233-238,1999$.

[100] T Cover and J Thomas. Elements of Information Theory. John Wiley \& Sons, New York, 1991. 
[101] S Amari, A Cichocki, and H Yang. A new learning algorithm for blind source separation. Advances in Neural Information Processing Systems, 8:757763, 1996.

[102] A J Bell and T J Sejnowski. An information maximization approach to blind separation and blind deconvolution. Neural Computation, 7(6):1129-1159, 1995.

[103] A Cichocki and S Amari. Adaptive Blind Signal Separation and Image Processing. John Wiley \& Sons, New York, 2002.

[104] L Molgedey and H G Schuster. Separation of a mixture of independent signals using time delayed correlations. Physical Review Letters, 72(23):36343637, 1994.

[105] S Van Gerden and D Van Compernolle. Parameter sensitivity in adaptive blind signal separation. In Proceeding Workshop on Adaptive Algorithms in Communications, pages 53-57, Bayonne, France, 1994.

[106] J M Sanchis, F Castells, and J J Rieta. Convolutive acoustic mixtures approximation to an instantaneous model using a stereo boundary microphone configuration. Lecture Notes in Computer Science, 3195:816-823, 2004. ISBN 4-540-23056-4.

[107] T M Mitchell. Machine Learning. McGraw-Hill International, 1997.

[108] K Torkola. Blind separation of convolved sources based on information maximization. In Proceeding of the IEEE Workshop on Neural Networks for Signal Processing, pages 343-346, 1996.

[109] T W Lee, M Girolami, and A J Sejnowski. A unifying information-theoretic framework for independent component analysis. Computers and Mathematics with Applications, 39:1-21, 2000.

[110] F Asano, S Ikeda, M Ogawa, H Asoh, and N Kitawaki. A combined approach of array processing and independent component analysis for blind separation of acoustic signals. In Proceedings of the IEEE Conference on Acoustics, Speech and Signal Processing, Salt Lake City, USA, 2001.

[111] S Haykin. Blind Deconvolution. Englewood Cliffs: Prentice Hall, 1994.

[112] G panci, P Campisi, S Colonnese, and G Scarano. Multichannel blind image deconvolution using the Bussgang algorithm: spatial and multiresolution approaches. IEEE Transactions on Image Processing, 12(11):1324 - 1337, 2003.

[113] R H Lambert. The FIR matrix toolbox. University of Southern California, 2001.

[114] S Ikeda and N Murata. A method of blind separation based on temporal structure of signals. In Proceedings of The Fifth International Conference on Neural Information Processing (ICONIP'98), pages 737-742, Kitakyushu, Japan, 1998. 
[115] N Murata, S Ikeda, and A Ziehe. An approach to blind source separation based on temporal structure of speech signals. Neurocomputing, 41(1-4):1$24,2001$.

[116] D Schobben and P Sommen. A new convolutive blind signal separation algortithm based on second order statistics. In Proc.Int.Conf on Signal and Image Processing, pages 564-569, 1998.

[117] D W E Schobben and P W Sommen. A frequency domain blind signal separation method based on decorrelation. IEEE Transactions on Signal Processing, 50(8):1855 - 1865, 2002.

[118] P J Podrid and P R Kowey. Cardiac Arrhythmia: Mechanisms, diagnosis and management. Lippincott Williams \& Wilkins, second edition, 2001.

[119] P S Addison. The Illustrated Wavelet Transform Handbook. Introductory Theory and Applications in Science, Engineering, Medicine and Finance. Institute of Physics Publishing, 2002.

[120] A Graps. An introduction to wavelets. IEEE Computational Science and Engineering, 2(2):1-18, 1995.

[121] S A Mallat. A Wavelet Tour of Signal Processing. Academic Press, second edition, 1999.

[122] C Sánchez, J J Rieta, F Castells, J Ródenas, and J Millet. Atrial activity extraction in holter registers using adaptive wavelet analysis. In Conf Proc IEEE Computers in Cardiology, volume 30, pages 569-572, 2003.

[123] R Alcaraz and J J Rieta. Wavelet bidomain sample entropy analysis to predict spontaneous termination of atrial fibrillation. Physiological Measurement, 29(1):65-80, 2008.

[124] A Bollmann, K Sonne, H D Esperer, I Toepffer, J J Langberg, and H U Klein. Non-invasive assessment of fibrillatory activity in patients with paroxys$\mathrm{mal}$ and persistent atrial fibrillation using the Holter ECG. Cardiovascular Research, 44:60-66, 1999.

[125] S M Pincus. Approximate entropy (ApEn) as complexity measure. Chaos, 5(1):110-117, 1995.

[126] S M Pincus. Assessing serial irregularity and its implications for health. Amn N Y Acad Sci, 954:245-267, 2001.

[127] F Auger, P Flandrin, P Gonçalvès, and O Lemoine. Time-Frequency Toolbox for use with MATLAB. Centre National de la Recherche Scientifique, 1996.

[128] R Bartels, J C Beatty, and B Barsky. An Introduction to Splines for Use in Computer Graphics and Geometric Modeling. Morgan Kauffman, London, 1987.

[129] E Nielson, J H Ahlberg, , and J Walsh. The Theory of Splines and Their Applications. Academic Press: New York, 1967. 
[130] L Cohen. Time-frequency distributions - a review. Proc. IEEE, 77:941-981, 1989.

[131] H Kantz and T Schreiber. Nonlinear time series analysis. Cambridge University Press, second edition, 2003.

[132] G L Baker and J P Gollub. Chaotic Dynamics. An introduction. Cambridge University Press, second edition, 1996.

[133] B Boucheham, Y Ferdi, and M C Batouche. Piecewise linear correction of ECG baseline wander: A curve simplification approach. Comput Methods Programs Biomed, 78(1):1-10, 2005.

[134] I Dotsinsky and T Stoyanov. Optimization of bidirectional digital filtering for drift suppression in electrocardiogram signals. J Med Eng Technol, 28(4):178-180, 2004.

[135] P S Hamilton, M Curley, and R Aimi. Effect of adaptive motion-artifact reduction on QRS detection. Biomed Instrum Technol, 34(3):197-202, 2000.

[136] Y Sun, K Chan, and S M Krishnan. ECG signal conditioning by morphological filtering. Comput Biol Med, 32(6):465-479, 2002.

[137] M Ferdjallah and R E Barr. Adaptive digital notch filter design on the unit circle for the removal of powerline noise from biomedical signals. IEEE Trans Biomed Eng, 41(6):529-536, 1994.

[138] S Araki and et al. The fundamental limitation of frequency domain blind source separation for convolutive mixture of speech. IEEE Transactions on Speech and Audio Processing, 11(2):109-116, 2003.

[139] P Langley, M Stridh, J J Rieta, L Sörnmo, J Mille-Roig, and A Murray. Comparison of atrial rhythm extraction techniques for the estimation of the main atrial frequency from the 12-lead electrocardiogram in atrial fibrillation. In Proc In .Conf on Computers in Cardiology, pages 29-32, 2002.

[140] A B Carlson. Communication Systems. An introduction to signal and noise in electrical communication. Mc-Graw-Hill International, third edition, 1986.

[141] M Stridh, A Bollmann, S B Olsson, and L Sörnmo. Detection and feature extraction of atrial tachyarrhythmias. IEEE engineering in medicine and biology, 25(6):31-39, 2006.

[142] R H Clayton and A Murray. Comparison of techniques for time-frequency analysis of the ECG during human ventricular fibrillation. IEE Proc.-Sei. Meas. Technol, 145(6):301-306, 1998.

[143] D E Lake, J S Richman, M P Griffin, and J R Moorman. Sample entropy analysis of neonatal heart rate variability. Am J Physiol, 283(3):R789-R797, 2002. 
[144] C J Huberty. Applied Discriminant Analysis. John Wiley and sons, Toronto, 1994.

[145] W J Krzanowski. Principles of Multivariate Analysis. Oxford University Press, 1988.

[146] G Boriani, I Diemberger, M Biffi, G Domenichini, C Martignani, C Valzania, and A Branzi. Electrical cardioversion for persistent atrial fibrillation or atrial flutter in clinical practice: predictors of long-term outcome. Int J Clin Pract, 61(5):748-756, 2007.

[147] Y Takahashi, P Sanders, and et al. Organization of frequency spectra of atrial fibrillation: Relevance to radiofrequency catheter ablation. J Cardiovasc Electrophysiol, 17(4):382-388, 2006.

[148] R Alcaraz. Application of Sample Entropy in the noninvasive study of atrial activity organization in atrial fibrillation. PhD thesis, Universidad Politécnica de Valencia, Valencia, Spain, 2008. 


\section{List of Figures}

2.1 (a) Location of the heart in the human body and (b) structure of the heart. . . . . . . . . . . . . . . . . . 10

2.2 Parts of the heart conduction system and their activation rates. . . 12

2.3 Different waveforms generated by the specialized cells heart conduction system in a normal ECG and the resultant body surface potential [50]. . . . . . . . . . . . . . . . . . . 13

2.4 Leads I, II and II constitute the Einthoven's triangle or 3-lead axial reference system. The augmented leads, $a V_{R}, a V_{L}$ and $a V_{F}$, in conjunction with I, II and III constitute the 6-lead axial reference system $[50] \ldots \ldots \ldots \ldots \ldots$

2.5 Location of the precordial leads on the chest. . . . . . . . . 16

2.6 Formation of the Wilson Central Terminal . . . . . . . . . . . . 17

2.7 Projection of the 12-lead ECG system in three orthogonal planes: frontal, transverse and sagital [50]. . . . . . . . . . . . . 18

2.8 Waves of the normal ECG at lead I [50]. . . . . . . . . . . . . 19

2.9 The generation of the ECG signal in the Einthoven limb leads [50]. 22

2.10 The generation of the ECG signal in the Einthoven limb leads (continued) $[50] . \ldots \ldots \ldots$

3.1 ECG of (a) Normal Sinus Rhythm (NSR) and (b) AF episode. In $\mathrm{AF}$, the $\mathrm{P}$ waves are substituted by rapid oscillations of fibrillatory waves and the ventricular response becomes irregular. . . . . . . 27 
3.2 Patterns of atrial fibrillation. (1) episodes that generally last less than or equal to 7 days (most less than $24 \mathrm{~h}$; (2) usually more than 7 days; (3) cardioversion failed or not attempted; and (4) either paroxysmal or persistent AF may be recurrent [8].

3.3 Principal electrophysiological mechanisms of atrial fibrillation. (a) Focal activation. The initiating focus (indicated by the asterisk) often lies within the region of the pulmonary veins. The resulting wavelets represent fibrillatory conduction, as in multiple-wavelet reentry. (b) Multiple-wavelet reentry. Wavelets (indicated by arrows) randomly reenter tissue previously activated by them or by another wavelet. The routes the wavelets travel vary. LA indicates left atrium; PV, pulmonary vein; ICV, inferior vena cava; SCV, superior vena cava; and $\mathrm{RA}$, right atrium $[1] \ldots \ldots \ldots$

3.4 a) A sinus impulse activates area A. (b) A premature beat arising in area $B$ fails to reach area $A$ because the intervening tissue remains refractory from the preceding sinus beat. (c) The premature stimulus travels slowly via an alternative route back to area A, allowing enough time for area $A$ to recover and be excited. (d) Area A re-excites area $B$, and the cycle sustains itself [58]. . . . . . . . .

3.5 Prevalence of AF in two American epidemiological studies. Framingham indicates the Framingham Heart Study; CHS, Cardiovascular Health Study [1] . . . . . . . . . . . . . . . . . . .

3.6 Incidence of atrial fibrillation in two American epidemiological studies. Framingham indicates the Framingham Heart Study, and CHS indicates Cardiovascular Health Study [1] . . . . . . . . . . . . 32

3.7 Independent risk factors for $\mathrm{AF}[70] \ldots \ldots \ldots \ldots$

3.8 Adverse effects of AF on QoL. Lower scores mean poorer QoL [4]. . 33

3.9 Integration surfaces and geometry outline involved in the forward problem solution when the observation point $i$ is placed on the inner bounding of the body surface $S_{B}[10] \ldots \ldots \ldots \ldots$

3.10 Integration surfaces and geometry outline involved in the forward problem solution when the observation point $i$ lies on the outer bounding of the heart surface $S_{H}[10] \ldots \ldots \ldots \ldots \ldots$

3.11 QRS complex detail of a 12-lead AF ECG. The maximum projection of the heart vector on each ECG lead occurs at different moments .

4.1 a) Computation of the QRST template of an AF episode by QRST beat averaging $b$ ) Estimation of the AA by aligning and subtracting the QRST template. . . . . . . . . . . . . . . . . . 
4.2 The Blind Source Separation problem. The $M$ observed signals are function of $N$ independent sources. The sources and the transformation functions are unknown. BSS algorithms try to approximate the separation system that inverts the process. In linear models, the mixing system and the separation system are represented by the $\mathbf{A}$ and the $\mathbf{W}$ matrices respectively. . . . . . . . . . . . .

4.3 Genesis of the standard 12-leads ECG as the linear mixture of atrial activity (AA), ventricular activity (VA), noise, and other bioelectric sources. . . . . . . . . . . . . . . . . . .

4.4 Instantaneous linear mixing model. Here the observations of the ECG are assumed to be instantaneous linear mixtures of the bioelectric sources. . . . . . . . . . . . . . . . . . . . . .

4.5 Convolutive linear mixing model. Here the observations of the ECG are assumed to be convolutive linear mixtures of the bioelectric sources. . . . . . . . . . . . . . . . . . . . . .

4.6 Ideal global matrix $\mathbf{G}$ with FIR filters length equal to 15 . The elements of the main diagonal, i.e. filters 1.1 and 2.2, are Dirac deltas. The rest of elements are filters of null response. . . . . . . . . . . 52

4.7 Example of histogram and kurtosis of a)AA source and b)VA source. The AA behaves as a subgaussian random variable with negative kurtosis. The VA behaves as a supergaussian random variable with possitive kurtosis. . . . . . . . . . . . . . . . . 55

4.8 Standard LMS Algorithm . . . . . . . . . . . . . . . . . . . 59

4.9 Example of convolution between an arbitrary signal and a wavelet function at two different scales, $a=1$ and $a=5$, and for different time shifts given by the translation parameter $b . \ldots \ldots 6$

4.10 Multi-Resolution Analysis by successive lowpass and highpass fil-

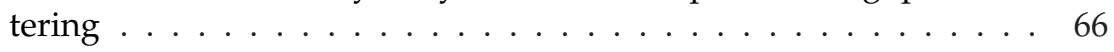

4.11 General CMBS process for AA extraction. . . . . . . . . . . . 67

4.12 Several mother wavelet functions used to transform Holter leads into a higher number of observations . . . . . . . . . . . 68

4.13 Decomposition of a Holter lead into 8 detail and one approximation coefficient by using symmlet 8 with 8 decomposition levels . . . 70

5.1 General process to classify a PAF episode as terminating or non-

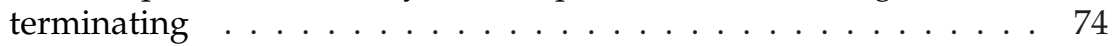


5.2 Time-frequency plot of a typical AA signal. a) AA spectrogram computed using Hamming windows of 1024 samples in length and $75 \%$ overlap. b) Spectrogram slice at $t=50$ seconds, interpolated fitting curve, local maxima and minima, and spectral features $f_{p 1}$, $f_{p 2}, A_{1}$ and $A_{2} \ldots \ldots \ldots \ldots \ldots \ldots \ldots \ldots \ldots \ldots$

5.3 (a) The threshold of the univariate analysis is fixed as the optimal decision level of SampEn for the learning set (b) The method is finally assessed by considering the recordings of the test set and the threshold fixed by the learning set. Black circles indicate terminating episodes. White circles indicate nonterminating episodes.

5.4 (a) The discriminant function is adjusted from the learning set (b) The method is finally assessed by considering the recordings of the test set and the adjusted discriminant function. Black circles indicate terminating episodes. White circles indicate nonterminating episodes. . . . . . . . . . . . . . . . . .

5.5 General process of analysis. Subindexes express the number of bits. (a) Quantization of leads, (b) AA extraction by the CMBS, (c) Low pass filtering, (d) Spectrogram of the quantized AA, (e) construction of $f_{p}$ and $S C$ sequences, and computation of the SampEn

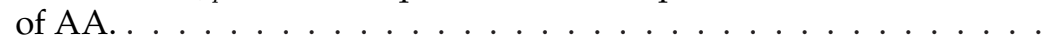

5.6 Example of the stroboscopic view of vector $x=\left[\begin{array}{lllllll}1 & 2 & 3 & 4 & 3 & 2 & 1\end{array}\right] \ldots \ldots$

6.1 Example of synthetic leads $x_{1}$ and $x_{2}$ formation from the AA and the VA. The filters of matrix $\mathbf{A}$ take the following values in the example: $h_{11}=\left[\begin{array}{ll}1 & 0.5\end{array}\right], h_{12}=\left[\begin{array}{ll}2 & 1\end{array}\right], h_{21}=\left[\begin{array}{ll}1 & -0.5\end{array}\right], h_{22}=$ $[-2-1] \ldots \ldots \ldots \ldots \ldots \ldots \ldots$

6.2 Second environment synthetic ECG generation. The AF 12-leads ECGs are synthesized by adding the AA and the VA of every lead, previously separated from real ECGs of AF episodes. . . . . . . . .

7.1 Mean $R_{A A_{t}}$ for the Infomax algorithm in the first environment. The length of the mixing matrix filters $N_{m}$ is varied from 1 to 8 and the length of the separation matrix filters $N_{s}$ from 2 to 32 . The values for the FastICA algorithm are also included for comparison.

7.2 Mean $\operatorname{SIR}_{A A}(d B)$ for the Infomax algorithm in the first environment. The length of the mixing matrix filters $N_{m}$ is varied from 1 to 8 and the length of the separation matrix filters $N_{s}$ from 2 to 32 . The values for the FastICA algorithm are also included for comparison. . . . . . . . . . . . . . . . . . . . . 104 
7.3 Mean $R_{A A_{t}}$ for the MBLMS algorithm in the first environment. The length of the mixing matrix filters $N_{m}$ is varied from 1 to 8 and the length of the separation matrix filters $N_{s}$ from 2 to 32. The values for the FastICA algorithm are also included for comparison. . . . . 105

7.4 Mean $S I R_{A A}(d B)$ for the MBLMS algorithm in the first environment. The length of the mixing matrix filters $N_{m}$ is varied from 1 to 8 and the length of the separation matrix filters $N_{s}$ from 2 to 32 . The values for the FastICA algorithm are also included for com-

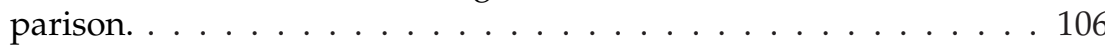

7.5 Mean $R_{A A_{t}}$ for the TDD algorithm in the first environment. The length of the mixing matrix filters $N_{m}$ is varied from 1 to 8 and the length of the separation matrix filters $N_{s}$ from 2 to 32. The values for the FastICA algorithm are also included for comparison. . . . . 108

7.6 Mean $S I R_{A A}(d B)$ for the TDD algorithm in the first environment. The length of the mixing matrix filters $N_{m}$ is varied from 1 to 8 and the length of the separation matrix filters $N_{s}$ from 2 to 32 . The values for the FastICA algorithm are also included for comparison. 108

7.7 Mean $R_{A A_{t}}$ for the CoBliSS algorithm in the first environment. The length of the mixing matrix filters $N_{m}$ is varied from 1 to 8 and the length of the separation matrix filters $N_{s}$ from 1 to 32. The values for the FastICA algorithm are also included for comparison. . . . . 110

7.8 Mean $S I R_{A A}(d B)$ for the CoBliSS algorithm in the first environment. The length of the mixing matrix filters $N_{m}$ is varied from 1 to 8 and the length of the separation matrix filters $N_{s}$ from 1 to 32 . The values for the FastICA algorithm are also included for com-

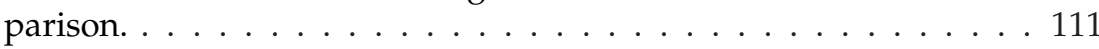

7.9 CBSS algorithms (a) $S I R_{A A}$ and (b) $R_{A A_{t}}$ mean values in the first environment as a function of the mixing matrix filters length $\left(N_{m}\right)$ regardless of the separation matrix filters length $\left(N_{s}\right) \ldots \ldots 113$

7.10 CBSS algorithms (a) $S I R_{A A}$ and (b) $R_{A A_{t}}$ mean values in the first environment as a function of the separation matrix filters length $\left(N_{s}\right)$ regardless of the mixing matrix filters length $\left(N_{m}\right) \ldots \ldots 114$

7.11 $S I R_{A A}$ and $R_{A A}$ mean values of the Infomax algorithm in the second environment as a function of the FIR filters length of the separation matrices $\left(\mathrm{N}_{s}\right)$. Seven different lengths of the filters were considered. . . . . . . . . . . . . . . 116

7.12 Mean $R_{A A_{t}}$ between the original AA and the one extracted by FastICA, WBS and CMBS from synthetic ECG recordings of the first environment at three levels of SNR. . . . . . . . . . . . . . . . . . . . 119 
7.13 Mean $R_{A A_{f}}$ between the original AA and the one extracted by FastICA, WBS and CMBS from synthetic ECG recordings of the first environment at three levels of SNR. . . . . . . . . . . . . . . 12

7.14 Mean $f_{p 1}$ of the AA extracted by FastICA, WBS and CMBS from synthetic ECG recordings of the first environment at three levels of SNR. . . . . . . . . . . . . . . . . 120

7.15 Mean $S C$ of the AA extracted by FastICA, WBS and CMBS from synthetic ECG recordings of the first environment at three levels of SNR.

7.16 Mean $f_{p 1}$ of the AA extracted by FastICA, WBS and CMBS from real ECG recordings of the second environment at three levels of SNR.

7.17 Mean $S C$ of the AA extracted by FastICA, WBS and CMBS from real ECG recordings of the second environment at three levels of SNR.

7.18 Results of the $t$-test for the $S a m p E n$ of all the spectral features. a) Mean and standard deviation of SampEn for groups N and T, b) $S a m p E n$ bilateral significance between groups. A feature is considered relevant when its bilateral significance is lower than 0.05 .

7.19 a) Type $\mathrm{T}$ and b) type $\mathrm{N} f_{p 1}$ series example with respective $S a m p E n$ values 0.0153 and 0.1556 . Higher $S a m p E n$ indicates lower regularity of the signal. . . . . . . . . . . . . . . . . 127

7.20 Learning set ROC curve fitted using the one-term exponential model for the SampEn of $f_{p 1}$. Decision point chosen for sensitivity $91 \%$ and 1 - specificity $14 \%$. . . . . . . . . . . . . . 12

7.21 Classification of type $\mathrm{N}$ and $\mathrm{T}$ episodes using a threshold value for the SampEn of $f_{p 1}$ equal to 0.1173 . The $95 \%$ of the learning set and $86.67 \%$ of the test set recordings were correctly classified. . . . . . . 129

7.22 Cross-correlation absolute values for the SampEn of the spectral significant features and the AA. Pairs of variables with high crosscorrelation do not contribute to the resultant discriminant model.

7.23 Two dimensional representations of the results taken by SampEn pairs of a) $A A$ and $f_{p 1}$, b) $A A$ and $\Delta f_{p}$, c) $f_{p 1}$ and $\Delta f_{p}$, and 3-D plot showing the hyperplane defined by the discriminant function. The $100 \%$ of the learning set recordings are classified correctly. In the test test, $93.75 \%$ of $\mathrm{N}$ recordings and $92.86 \%$ of $\mathrm{T}$ recordings are correctly classified. . . . . . . . . . . . . . . . . . 133 
7.24 $R_{q}$ between the 16-bit AA signal $A A_{16}$ and $A A_{q}$ encoded with 4 to 15 bits. Results are given for non-filtered $A A_{q}$ and for low-pass filtered $A A_{q}$. . . . . . . . . . . . . . . . . . . 137

7.25 Mean MAE of $f_{p 1}$ for a number of encoding bits from 4 to 15 . Higher values of MAE denote a worse approximation of the spectral feature $f_{p 1}$.

7.26 Mean MAE of $S C$ for a number of encoding bits from 4 to 15 . Higher values of MAE denote a worse approximation of the spectral feature $S C$.

7.27 Mean $M A E$ of $A A$ SampEn for a number of encoding bits from 4 to 15 . Higher values of MAE denote a worse approximation of the AA SampEn. . . . . . . . . . . . . . . . . 140

7.28 Example of $A_{1}$ phase portrait of a PAF episode belonging to type $\mathrm{N}$ group. No particular features of these plots can be attributed to the recording type. . . . . . . . . . . . . . . . 14

7.29 Example of $A_{1}$ phase portrait of a PAF episode belonging to type $\mathrm{T}$ group. No particular features of these plots can be attributed to the recording type. . . . . . . . . . . . . . 142

7.30 Example of $f_{p 1}$ phase portrait of a PAF episode belonging to type $\mathrm{N}$ group. Dot clusters are far from the bottom left-hand corner of the graph. . . . . . . . . . . . . . . . 143

7.31 Example of $f_{p 1}$ phase portrait of a PAF episode belonging to type $\mathrm{T}$ group. Dot clusters are concentrated close to the bottom left-hand corner of the graph. . . . . . . . . . . . . . . . . . . . 143

7.32 Example of $A_{2}$ phase portrait of a PAF episode belonging to type $\mathrm{N}$ group. No particular features of these plots can be attributed to the recording type. . . . . . . . . . . . . . 14

7.33 Example of $A_{2}$ phase portrait of a PAF episode belonging to $\mathrm{f}$ the type T group. No particular features of these plots can be attributed to the recording type. . . . . . . . . . . . . . . . 144

7.34 Example of $f_{p 2}$ phase portrait of a PAF episode belonging to type $\mathrm{N}$ group. No particular features of these plots can be attributed to the recording type. . . . . . . . . . . . . 145

7.35 Example of $f_{p 2}$ phase portrait of a PAF episode belonging to type $\mathrm{T}$ group. No particular features of these plots can be attributed to the recording type. . . . . . . . . . . . . . 145 



\section{List of Tables}

3.1 Prognosis of patients with AF comorbidities. HF, heart failure; CHF, congestive heart Failure; MI, myocardial infarction. . . . . . . 33

4.1 Analyzed wavelet mothers and their levels of decomposition. . . . 68

5.1 Several usual kernel functions of time-frequncy distributions . . . . 79

6.1 Tested filter lengths of the mixing and separation matrices in the first environment. $N_{m}$ is the length of the mixing matrix filters. $N_{s}$ is the length of the separation matrix filters. . . . . . . . . . . . . 89

7.1 Infomax mean $R_{A A_{t}}$ of Figure 7.1 expressed with three significant

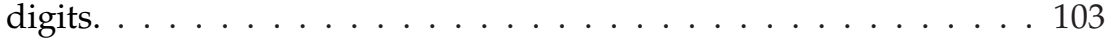

7.2 Infomax mean $S I R_{A A}(d B)$ of Figure 7.2 expressed with one significant decimal. . . . . . . . . . . . . . . . . . . . . . . . . 104

7.3 MBLMS mean $R_{A A_{t}}$ of Figure 7.3 expressed with trhee significant digits. . . . . . . . . . . . . . 106

7.4 MBLMS mean $S I R_{A A}(d B)$ of Figure 7.4 expressed with one significant decimal. . . . . . . . . . . . . . . . . . 106

7.5 TDD mean $R_{A A_{t}}$ of Figure 7.5 expressed with trhee significant digits.107

7.6 TDD mean $S I R_{A A}(d B)$ of Figure 7.6 expressed with one significant decimal. . . . . . . . . . . . . . . . . . . . . . 109

7.7 CoBliSS mean $R_{A A_{t}}$ of Figure 7.7 expressed with trhee significant digits. . . . . . . . . . . . . . . 110

7.8 CoBliSS mean $S I R_{A A}(d B)$ of Figure 7.8 expressed with one significant decimal. . . . . . . . . . . . . . . . . . . 111 
7.9 CBSS algorithms mean $S I R_{A A}$ in the first environment as a function of $N_{m}$ regardless of $N_{s}$ expressed with one significant decimal. 113

7.10 CBSS algorithms mean $R_{A A_{t}}$ in the first environment as a function of $N_{m}$ regardless of $N_{s}$ expressed with three significant digits. . . 114

7.11 CBSS algorithms mean $S I R_{A A}$ in the first environment as a function of $N_{s}$ regardless of $N_{m}$ expressed with one significant decimal. 114

7.12 CBSS algorithms mean $R_{A A_{t}}$ in the first environment as a function of $N_{s}$ regardless of $N_{m}$ expressed with three significant digits. . . 115

7.13 SIR $R_{A A}$ and $R_{A A_{t}}$ mean values of the Infomax algorithm as a function of $N_{s}$ in the second environment. . . . . . . . . . 116

7.14 CMBS mean values of $R_{A A_{t}}, R_{A A_{f}}, f_{p 1}(H z)$ and $S C$ for different wavelet mother functions used in the implementation of the CMBS wavelet decomposition stage. . . . . . . . . . . . . . . . . 121

7.15 Mean $f_{p 1}$ of CMBS and ABS algorithms in the second environment. 123

7.16 Mean $S C$ of CMBS and ABS algorithms in the second environment. 124

7.17 SampEn of $f_{p 1}$ and bilateral significance between groups $\mathrm{N}$ and $\mathrm{T}$ computed for nine time-frequency distributions: spectrogram (SP), Wigner-Ville (WV), pseudo-Wigner-Ville (PWV), Margeneau-Hill (MH), pseudo-Margeneau-Hill (PMH), Page (PG), pseudo-Page (PPG), Zhao-Atlas-Marks (ZAM) and Choi-Williams (CW) . . . . . . . . 124

7.18 SampEn of $S C$ and bilateral significance between groups $\mathrm{N}$ and $\mathrm{T}$ computed for nine time-frequency distributions: spectrogram (SP), Wigner-Ville (WV), pseudo-Wigner-Ville (PWV), Margeneau-Hill (MH), pseudo-Margeneau-Hill (PMH), Page (PG), pseudo-Page (PPG), Zhao-Atlas-Marks (ZAM) and Choi-Williams (CW) . . . . . . . 125

7.19 SampEn of $f_{p 1}$ bilateral significance for different tested values of $m$ and $r$. The values $m=2$ and $r=0.25 S T D$ are used in the study for $S a m p E n$ of $f_{p 1}$ regarding to the minimum associated bilateral significance.

7.20 Area under ROC curve for the SampEn of the learning set relevant spectral features. . . . . . . . . . . . . . . . . 127

7.21 SampEn of $f_{p 1}$ for each recording of the learning set. The $a_{N}$ recordings belong to the nonterminating group, and the $a_{T}$ recordings to the terminating group. A classification was made attending to the threshold value of 0.1173 . Highlighted recordings were misclassified. . . . . . . . . . . . . . . . . . . 128 
7.22 SampEn of $f_{p 1}$ for each recording of the test set. A classification was made attending to the threshold value of 0.1173 . Highlighted recordings were misclassified. . . . . . . . . . . . . . . . . . 129

7.23 Correlations between the SanpEn of variables considered in the stepwise analysis. . . . . . . . . . . . . . . . . . . 130

7.24 Variables in the stepwise analysis. The statistic $F$ indicates which variable must be added to the model in each step. . . . . . . . . . . 132

7.25 Standardized canonical discriminant function coefficients from the stepwise analysis. . . . . . . . . . . . . . . . . 132

7.26 SamEn values of $f_{p 1}, \Delta f_{p}$, and $A A$ for each recording of the learning set used in the discriminant analysis. . . . . . . . . . . . 134

7.27 SamEn values of $f_{p 1}, \Delta f_{p}$, and $A A$ for each recording of the test set used in the discriminant analysis. Highlighted recordings were misclassified. . . . . . . . . . . . . . . . . . . . . 135

7.28 Classification of learning and test AF ECG recordings as type $\mathrm{N}$ or type $\mathrm{T}$ by using the discriminant analysis. . . . . . . . . . . 136

7.29 Mean $R_{q}$ between $A A_{16}$ and the $A A_{q}$ encoded with 4 to 15 bits. Results are given for non-filtered $A A_{q}$ and for low-pass filtered $A A_{q} .138$

7.30 Mean MAE of $f_{p 1}, \mathrm{SC}$, and AA SampEn for $A A_{q}$ encoded with 4 to 15 bits. . . . . . . . . . . . . . . . . . . . 139

7.31 Learning set recordings evaluated by Poincaré plots of $f_{p 1}$. Highlighted recordings were misclassified. . . . . . . . . . . . 146

7.32 Test set recordings evaluated by Poincaré plots of $f_{p 1}$. Highlighted recordings were misclassified. . . . . . . . . . . . . . 146 



\title{
Mathematical Notation
}

\author{
$a_{i j}$ Coefficients of the mixing matrix in the instantaneous mixing \\ model \\ $a_{i} \quad i^{\text {th }}$ approximation coefficient in Wavelet transform \\ $\vec{A} \quad$ Magnetic potential vector \\ A Mixing matrix \\ $A A_{q} \quad$ AA quantized with $q$ bits \\ $A A_{16} \quad$ AA quantized with 16 bits \\ $A_{1} \quad$ Main peak magnitude \\ $A_{2} \quad$ Second largest peak magnitude \\ $\bar{A}_{2} \quad$ Normalized amplitude of the second largest peak \\ $a V_{R}, a V_{L}, a V_{F} \quad$ Voltage of augmented limb leads \\ $C_{a}(b) \quad$ Continuous wavelet transform of scale $a$ and translation $b$ \\ $C_{x}(t, \omega) \quad$ Any Cohen's class time-frequency distribution \\ $d S \quad$ Differential surface \\ $d_{i} \quad i^{\text {th }}$ detail coefficient in Wavelet transform \\ $d_{1}, d_{2}$ Deviation of the main and second peak frequencies from their \\ respective mean values \\ $d\left(X_{m}(i), X_{m}(j)\right) \quad$ Distance between patterns $X_{m}(i)$ and $X_{m}(j)$ \\ $d V \quad$ Differential volume \\ $\Delta f_{p} \quad$ Normalized distance between $f_{p 1}$ and $f_{p 2}$ \\ $e, \mathbf{e} \quad$ Error signal and vector of error signals
}




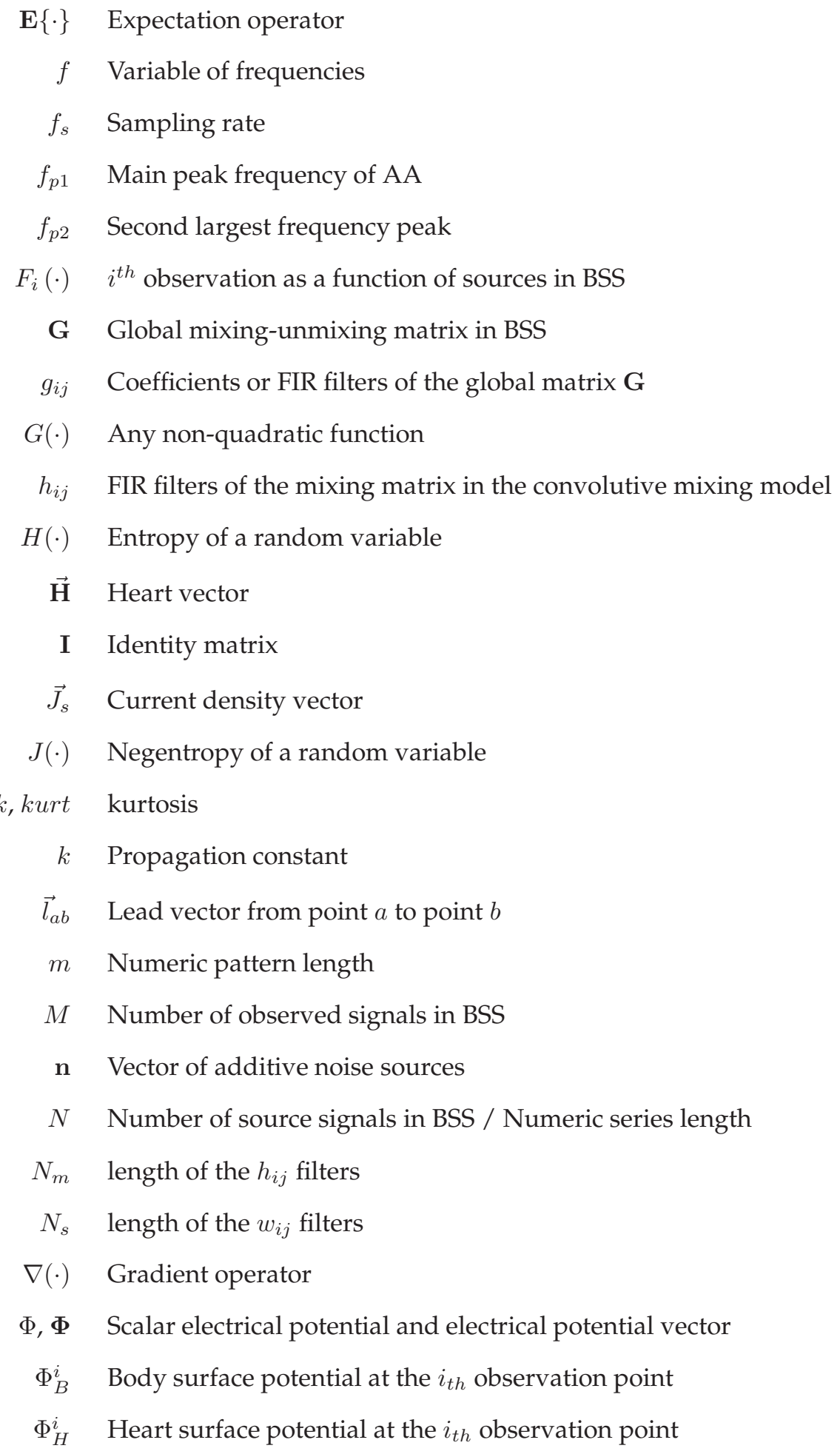


$\Phi_{L}, \Phi_{R}, \Phi_{L} \quad$ Potential of left arm, right arm, and left foot, respectively

$\Phi_{C T} \quad$ Potential of the Wilson Central Terminal

$\Phi(\theta, \tau) \quad$ Any Cohen's class kernel function

$\Psi(t) \quad$ Mother wavelet function

$\Psi_{a, b}(t) \quad$ Wavelet function of scale $a$ and translation $b$

$\Psi\left(y_{i}(n)\right) \quad$ Cost function of $y_{i}(n)$

$\Gamma_{H}, \Gamma_{H}$ Scalar electric potential gradient and potential gradient vector

$\mu \quad$ Magnetic permeability

$\vec{n}_{S} \quad$ Normal unitary vector on surface $S$

$p b 3 d B$ power in the $3-\mathrm{dB}$ band

$r$ Distance for the criterion of similarity between patterns

$\vec{r}_{P Q} \quad$ Position vector between a point in surface $\mathrm{P}$ and a point in surface $Q$

$R, \mathbf{R} \quad$ Correlation index and correlation matrix

$R_{A A_{t}} \quad$ Correlation between $s_{A A}$ and $\hat{s}_{A A}$ in time domain

$R_{A A_{f}} \quad$ Correlation between $s_{A A}$ and $\hat{s}_{A A}$ in frequency domain

$R_{q} \quad$ Cross-correlation between $A A_{16}$ and $A A_{q}$

$s_{j}, \hat{s}_{j} \quad$ Original and estimated $j^{\text {th }}$ source BSS

$s_{A A} \quad$ Original atrial activity signal

$\hat{s}_{A A} \quad$ Estimated atrial activity

$\mathbf{s}, \hat{\mathbf{s}} \quad$ Original and estimated vector of sources in BSS

$S_{B} \quad$ Body surface

$S_{H} \quad$ Heart surface

$d \vec{S}_{H} \quad$ Outward differential normal vector to $S_{H}$

$S I R_{A A}$ Improvement of the Signal to Interference Ratio of AA accomplished by BSS algorithms

$\mathbf{T}_{B H} \quad$ Matrix of potential transfer coefficients between heart and body surfaces

$u_{a b} \quad$ Potential measured in an arbitrary lead that comprises points $a$ and $b$ 


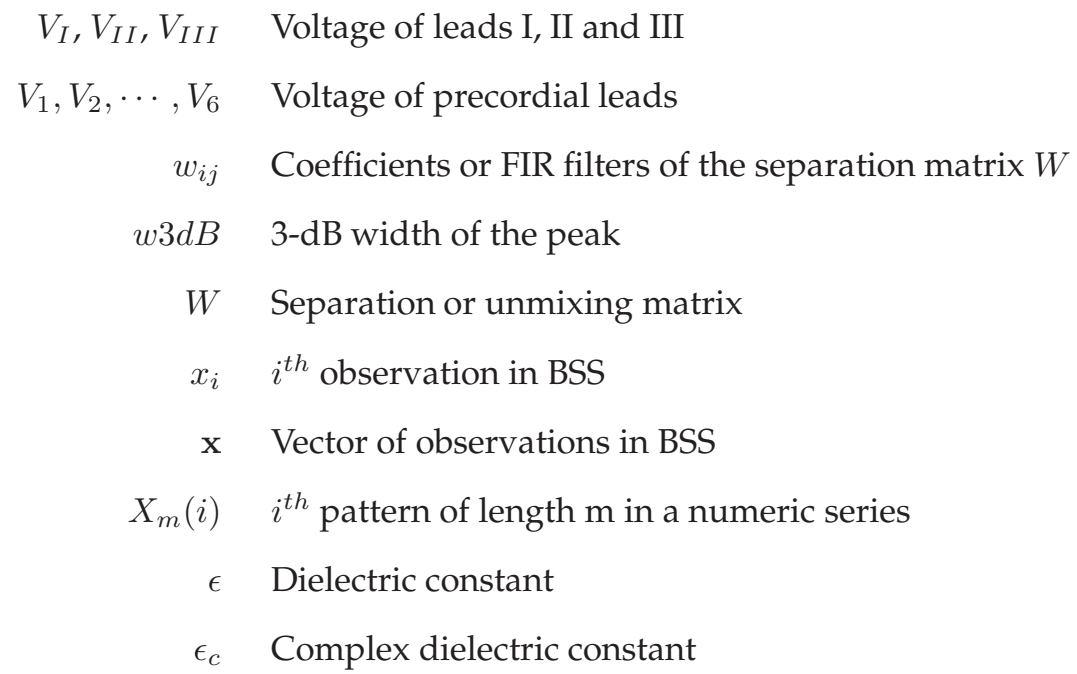

$\Lambda \quad$ Eigenvalues matrix

$\rho \quad$ Electric charge density

$\sigma \quad$ Electric conductivity

$\sigma_{c} \quad$ Complex electric conductivity

$\omega$ Pulsation

$\Omega \quad$ Solid angle

* Convolution operator 


\section{List of Acronyms}

\begin{tabular}{|c|c|}
\hline AA & Atrial Activity \\
\hline ABS & Average Beat Subtraction \\
\hline $\mathrm{AF}$ & Atrial Fibrillation \\
\hline ApEn & Approximate Entropy \\
\hline ASVC & Adaptive Singular Value Cancellation \\
\hline AV & Atrioventricular \\
\hline AWGN & Additive White Gaussian Noise \\
\hline BSS & Blind Source Separation \\
\hline CBSS & Convolutive Blind Source Separation \\
\hline CHS & Cardiovascular Heart Study \\
\hline $\mathrm{CHF}$ & Congestive heart failure \\
\hline CMBS & Convolutive Multiband Blind Separation \\
\hline CoBliSS & Convolutive Blind Signal Separation \\
\hline CMRR & Common-mode rejection ratio \\
\hline $\mathrm{CW}$ & Choi-Williams \\
\hline CWT & Continuous Wavelet Transform \\
\hline DWT & Discrete Wavelet Transform \\
\hline DyWT & Dyadic Discrete Wavelet Transform \\
\hline ECG & Electrocardiogram \\
\hline$f$ waves & Fibrillatory waves \\
\hline FFT & Fast Fourier Transform \\
\hline FIR & Finite impulse response \\
\hline $\mathrm{HF}$ & Heart failure \\
\hline $\mathrm{HV}$ & Heart vector \\
\hline HOS & High order statistics \\
\hline ICA & Independent Component Analysis \\
\hline $\mathrm{ICV}$ & Inferior vena cava \\
\hline IIR & Infinite impulse response \\
\hline IT & Information Theory \\
\hline LA & Left atrium \\
\hline LED & Light emitting diode \\
\hline LMS & Least-Mean Square \\
\hline MH & Margenau-Hill \\
\hline MF & Median frequency \\
\hline
\end{tabular}




\begin{tabular}{|c|c|}
\hline MI & Myocardial infarction \\
\hline MBLMS & Multi-channel Blind Least-Mean Square \\
\hline MPSE & Multi-Parametric Sample Entropy \\
\hline MRA & Multi-Resolution Analysis \\
\hline MAE & Mean absolute error \\
\hline MIMO & Multiple input multiple output \\
\hline MSE & Mean square error \\
\hline $\mathrm{N}$ & Non-terminating \\
\hline NSR & Normal sinus rhythm \\
\hline PAF & Paroxysmal atrial fibrillation \\
\hline PV & Pulmonary vein \\
\hline PCA & Principal Component Analysis \\
\hline $\mathrm{pdf}$ & probability density function \\
\hline PG & Page \\
\hline PMH & pseudo-Margenau-Hill \\
\hline PPG & pseudo-Page \\
\hline PWV & pseudo-Wigner-Ville \\
\hline PSD & Power spectral density \\
\hline QoL & Quality of life \\
\hline QRST & QRS complex and T wave \\
\hline RA & Right atrium \\
\hline ROC & Receiver Operative Characteristic \\
\hline SampEn & Sample Entropy \\
\hline SA & Sinoatrial \\
\hline $\mathrm{SC}$ & Spectral Concentration \\
\hline SCV & Superior vena cava \\
\hline SIR & Signal to interference ratio \\
\hline SNR & Signal to noise ratio \\
\hline SOS & Second order statistics \\
\hline SP & Spectrogram \\
\hline SNR & Signal to noise ratio \\
\hline STD & Standard deviation \\
\hline $\mathrm{T}$ & Terminating \\
\hline TDD & Time-Delayed Decorrelation \\
\hline TMS & Template Matching and Subtraction \\
\hline VA & Ventricular Activity \\
\hline WBS & Wavelet Blind Separation \\
\hline WT & Wavelet Transform \\
\hline WCT & Wilson Central Terminal \\
\hline WV & Wigner-Ville \\
\hline ZAM & Zhao-Atlas-Marks \\
\hline
\end{tabular}

